\title{
Development Of A Modular
}

\section{Ratiometric Fluorescent Probe}

\section{For The Detection Of Extracellular}

\section{Superoxide}

\section{Doctoral Thesis}

Author(s):

Andina, Diana Laura Romana

Publication date:

2017

Permanent link:

https://doi.org/10.3929/ethz-b-000000281

Rights / license:

In Copyright - Non-Commercial Use Permitted 
DISS. ETH NO. 24338

\title{
DEVELOPMENT OF A MODULAR RATIOMETRIC FLUORESCENT PROBE FOR THE DETECTION OF EXTRACELLULAR SUPEROXIDE
}

\author{
A thesis submitted to attain the degree of \\ DOCTOR OF SCIENCES of ETH ZURICH \\ (Dr. sc. ETH Zurich) \\ presented by
}

\section{DIANA LAURA ROMANA ANDINA}

MSc ETH Chemistry, ETH Zürich

born on 27.09.1988

citizen of Croglio, TI, Switzerland

accepted on the recommendation of

Prof. Dr. Jean-Christophe Leroux, examiner

Prof. Dr. Paola Luciani, co-examiner

Prof. Dr. Pablo Rivera-Fuentes, co-examiner 



\section{Summary}

Reactive oxygen species (ROS) have undergone an exceptional change in the way they are perceived over the last two decades. Their role as simple antimicrobial agents during host defense has been broadened by a vast repertoire of signaling functions in health, disease and aging. Unravelling these short-lived species' diverse actions has concurrently increased the interest in them, emphasizing the need for reliable, specific and sensitive detection methods. Ratiometric fluorescent probes offer an elegant option to detect the analyte of interest and to normalize the obtained ROS-dependent signal to a second fluorescence emission. The ratio of the two signals is thus independent of the sensor concentration, and minimizes artefacts and intensity fluctuations. This Ph.D. thesis focuses on the design of a ratiometric probe for the detection of ROS in the extracellular space by linking a ROS-sensor (namely HCy5) and a reporter dye (Chromis500) with a well-defined, highly negatively charged macromolecule. As a further step, the linker was improved with a double-stranded DNA-analogue to produce a modular platform for ratiometric ROS detection.

In Chapter 1 ROS production is discussed in general and the action of neutrophils during intestinal inflammation is outlined.

Chapter 2 focuses on the advances in the field of ratiometric fluorescent probes for the detection of ROS and reactive nitrogen species. A summary of the probes published to date is provided, together with a critical discussion of their possible application in tissue culture and in vivo.

Before designing the ratiometric probe, the already existing ROS-sensor HCy5 was validated in cell-free and cell culture-based experiments relevant for the scope of this thesis, which are reported in Chapter 3. The detection of superoxide and hydroxyl radicals was successfully achieved in cell-free experiments and ROS-sensing in cell-culture based assays was exemplified. The sensing capacity of $\mathrm{HCy} 5$ was compared to other frequently used fluorescent ROS-sensors and it was found to be similar. Subsequently, to obtain an extracellular ROSsensor, this intracellular indicator was modified with hydrophilic macromolecules.

A ratiometric fluorescent sensor was created by coupling Chromis500 to the hydrophilic linker as outlined in Chapter 4. For this purpose, the extracellular indicator that consisted of ten poly(glutamic acid) residues, was modified with a lysine residue to enable coupling of the reporter dye. By measuring the Förster resonance energy transfer efficiency, the optimal distance between the two fluorophores was determined, and six discrete PEG10 units were found 
to reduce the effect sufficiently. Uptake studies in a cell line mimicking the intestinal epithelial barrier proved the predominantly extracellular nature of the ratiometric probe. Finally, specific superoxide sensing was exemplified in a cell-free assay and an approximately 2-fold increase in fluorescence emission ratio was detected.

In Chapter 5, a double stranded peptide nucleic acid was introduced in the linker design. This allowed us to increase the modularity of the probe and also to manipulate the two fluorophores separately. After obtaining the fully assembled ratiometric probe, its cellular uptake was assessed and its internalization was found to be greatly reduced compared to the uncoupled Cy5 and Chromis500. As for the previous approach, the superoxide sensing was successfully investigated in cell-free assays and a 2-fold increase in fluorescence emission ratio was obtained. The analysis was completed by ROS sensing in cell culture, which also resulted in a doubling of the emission ratio.

The main findings of this Ph.D. work are outlined in Chapter 6, where the conclusions drawn from the main results are presented, together with a discussion of the system's limitations and possible improvements. 


\section{Zusammenfassung}

Das Verständnis der Bedeutung reaktiver Sauerstoffspezies (RSS) hat sich in den letzten zwei Jahrzenten stark verändert. Während diese kurzlebigen Moleküle früher vornehmlich im Zusammenhang mit Entzündungen und für ihre antimikrobielle Aktivität bekannt waren, umfassen neurer Kenntnisse auch deren Rolle in der Signalübertragung in verschiedenen Geweben und bei Alterungsprozessen. Somit besteht heute ein grosses Interesse, diese Moleküle noch eingehender zu untersuchen. Der Nachweis von RSS stellt jedoch immer noch eine Herausforderung dar, was die Suche nach zuverlässigen, spezifischen und sensitiven Nachweismethoden intensiviert hat. Ratiometrisch auswertbare Fluoreszenzfarbstoffe scheinen für den Nachweis von RSS besonders vorteilhaft, da sie nicht nur den Analyten (RSS) direkt sichtbar machen können, sondern das erhaltene Signal mit einer zweiten Fluoreszenzemission zu normalisieren vermögen. Da das Verhältnis der beiden Fluoreszenzsignale unabhängig von der Konzentration des Fluoreszenzfarbstoffes ist, werden fluktuierenden Fluoreszenzemissionen und andere Artefakte vermieden. Die vorliegende Doktorarbeit befasst sich hauptsächlich mit der Entwicklung eines ratiometrischen Fluoreszenzfarbstoffs für den Nachweis von extrazellulären. Dazu wurde der bereits bekannte RSS-affine, intrazellulär aktive Farbstoff (HCy5) mit einem RSS-blinden Fluorophor (Chromis500) durch ein hydrophiles Makromolekül verbunden. Das makromolekulare Bindungsmolekül wurde in einem zweiten Schritt mit einem Doppelstrang eines DNS-Analogons modifiziert, womit ein flexibel kombinierbarer und ratiometrisch auswertbarere Farbstoff zur Verfügung stand.

In Kapitel 1 wird die physiologische RSS-Produktion grundsätzlich erklärt und für den Fall einer Entzündung im Darm spezifiziert.

Kapitel 2 fokussiert auf ratiometrisch auswertbare Fluoreszenzfarbstoffe, die RSS oder reaktive Stickstoffspezies sichtbar machen können. Bisher beschriebene Farbstoffe werden tabelliert und an einigen Beispielen wird deren mögliche Anwendung in Zellkulturen und in vivo kritisch diskutiert.

Experimentell wird der bekannte, intrazellulär aktive RSS-Farbstoff HCy5 zunächst in, im Zusammenhang mit der Doktorarbeit relevanten, zellfreien Medien und in Zellkulturen validiert (Kapitel 3). Der Nachweis von Superoxid- und Hydroxylradikalen in zellfreien Untersuchungen gelang und in einem Vergleich des Nachweisvermögens von HCy5 mit jenem anderer etablierter RSS-Sensoren wurden in verschiedenen Zellexperimenten ähnliche Ergebnisse 
erzielt. Danach wurde der intrazellulär aktive Farbstoff HCy5 mit hydrophilen Makromolekülen modifiziert, um einen extrazellulären RSS-Sensor zu erhalten.

Kapitel 4 beschreibt die Synthese eines ratiometrisch auswertbaren Fluoreszenzfarbstoffes zum Nachweis von RSS. Dazu wurde HCy5 zunächst mit einer Oligomerkette von zehn Glutaminsäuren (Bindungsmolekül) und einem Lysin modifiziert; an letztere Aminosäure konnte danach das RSS-blinde Chromis500 gebunden werden. Weiter wurde die Distanz zwischen den Fluorophoren optimiert, um die Förster-Resonanzenergietransfer (FRET) Effizienz zu minimieren, was durch Einfügen von sechs einzelnen PEG $_{10}$-Oligomeren beim Bindungsmolekül gelang. Die extrazelluläre Aktivität des Farbstoffes wurde durch Zellaufnahmeexperimente mit einer von Darmepithelzellen abgeleiteten Zelllinie bestätigt. Schliesslich wurde die spezifische Superoxid Detektionskapazität in zellfreien Experimenten untersucht und eine 2-fache Zunahme des ratiometrischen Verhältnisses der Fluoreszenz Emissionen beobachtet.

Kapitel 5 beschreibt die Optimierung des aminosäure-basierten Bindungsmoleküls, indem es mit einem Doppelstrang einer Peptidnukleinsäure modifiziert wurde. Diese Änderung im Bindungsmolekül erhöhte die Flexbilität des Sensors und ermöglichte die voneinander unabhängige Synthese und Handhabung der zwei Fluorophore. Wie für den vorhergehenden Ansatz wurde zuerst die Aufnahme in Zellen untersucht; im Vergleich zu den nativen Farbstoffen Cy5 und Chromis500 wurde das Farbstoffkonjugat in deutlich geringerem Ausmass internalisiert. In zellfreien Experimenten mit Superoxid konnte wiederum, wie in Kapitel 4 beschrieben, eine 2-fache Zunahme des Verhältnisses der Fluoreszenzemissionen gemessen werden. Die Untersuchungen wurden mit RSS-Messungen an Zellen ergänzt, wo ebenfalls eine Verdoppelung des Fluoreszenz-Emissionsverhältnisses beobachtet wurde.

Die wichtigsten Erkenntnisse dieser Doktorarbeit sind im Kapitel 6 zusammengefasst. Mögliche Einschränkungen des Systems werden diskutiert und Verbesserungsvorschläge gemacht. 


\section{Table of Content}

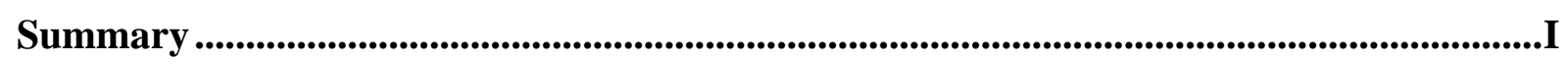

Zusammenfassung ............................................................................................................................II

Chapter 1: Background and Purpose..................................................................................................... 1

Chapter 2: Ratiometric Fluorescent Probes to Detect Reactive Oxygen Species............... 9

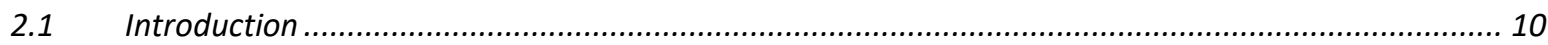

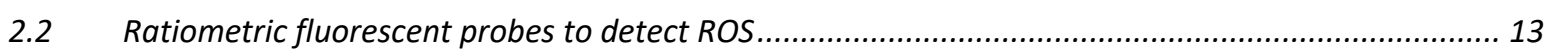

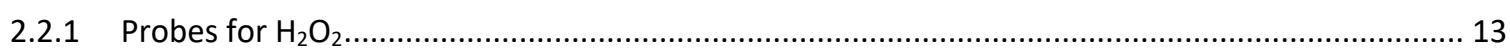

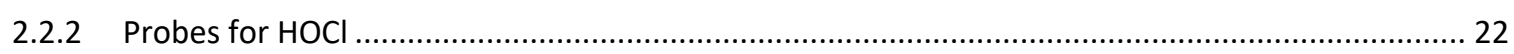

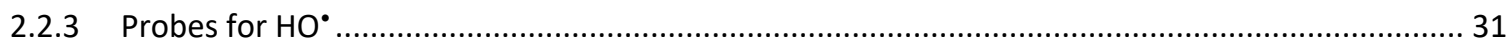

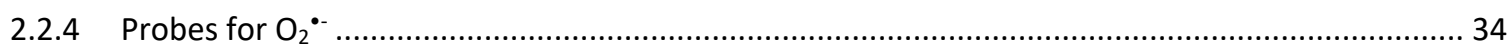

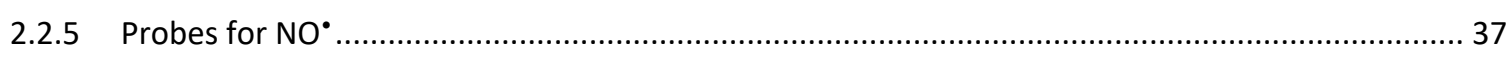

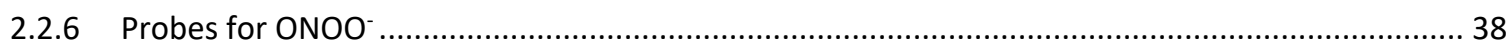

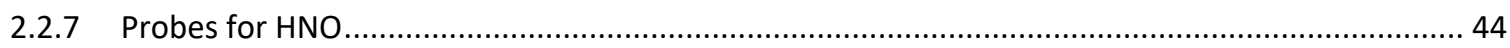

2.3 Ratiometric fluorescent probes to detect $h$ ROS and multiple ROS ..............................................44

2.4 Ratiometric fluorescent probes to monitor the redox-state .................................................... 48

2.5 Conclusion

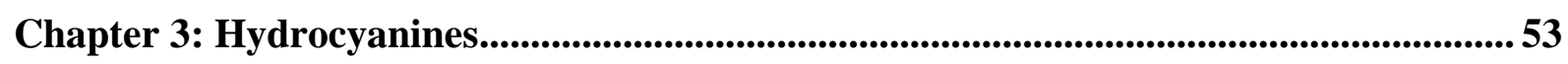

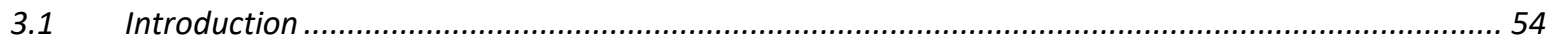

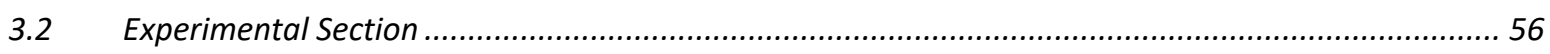

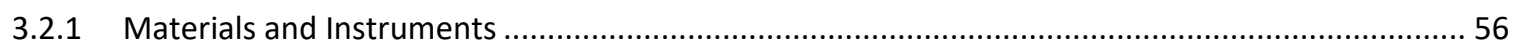

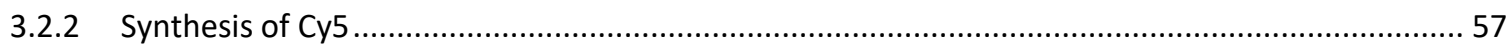

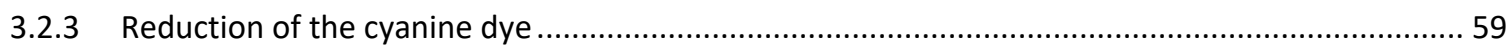

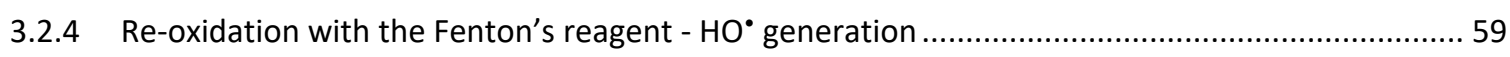

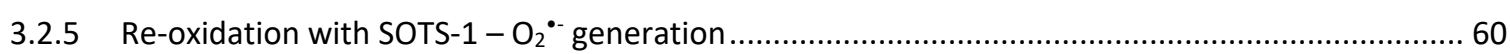

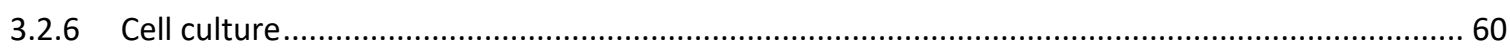

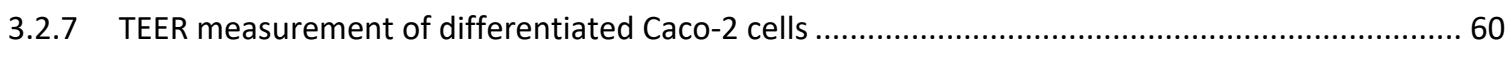

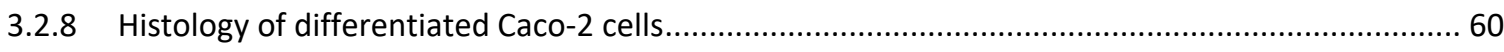

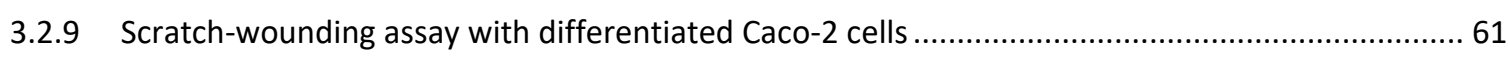

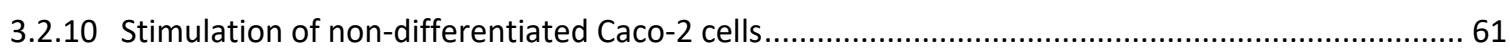

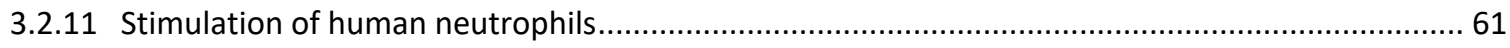

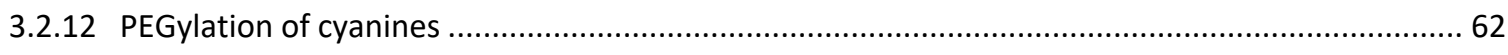




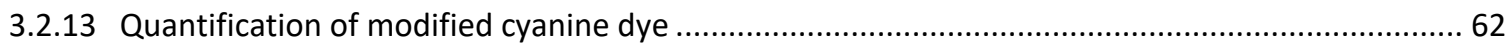

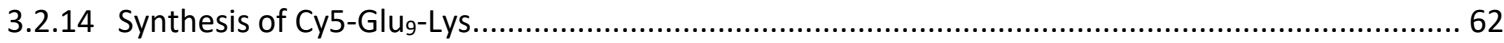

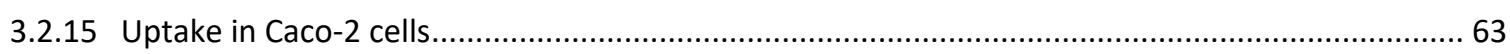

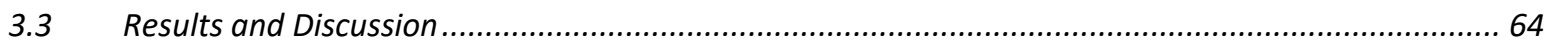

3.3.1 Screening of different commercial cyanine dyes with Fenton's reagent .................................64 64

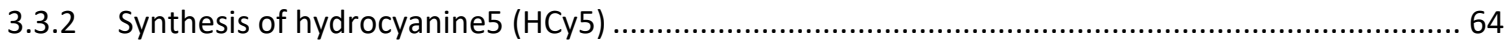

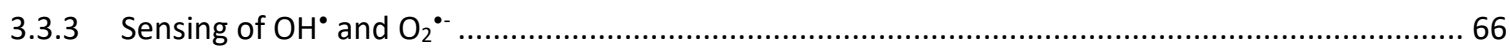

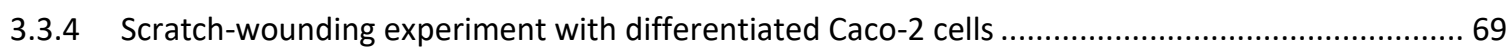

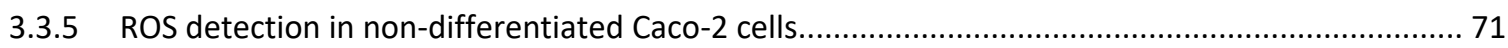

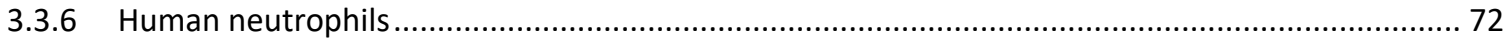

3.3.7 Modification of cyanine dyes and uptake in differentiated Caco-2 cells.................................... 73

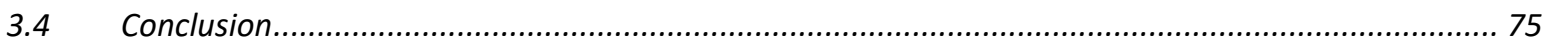

Chapter 4: Chromis-PGA-(PEG 10$)_{\mathrm{x}}-(\mathrm{H}) \mathrm{Cy5}$......................................................................... 77

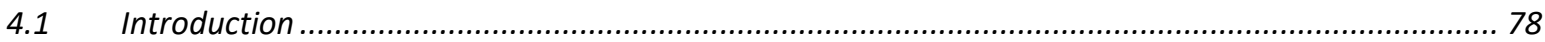

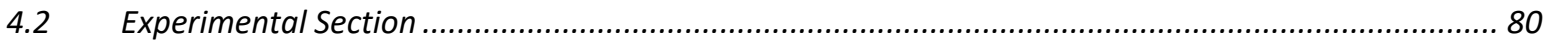

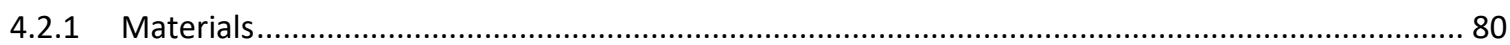

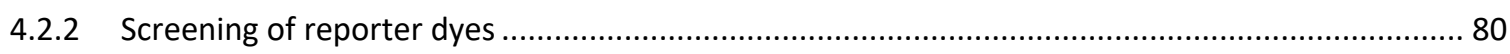

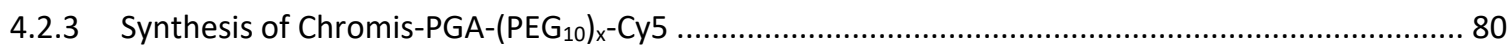

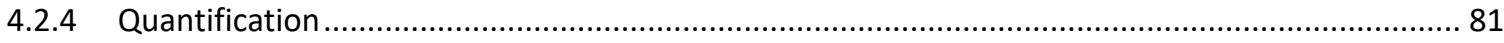

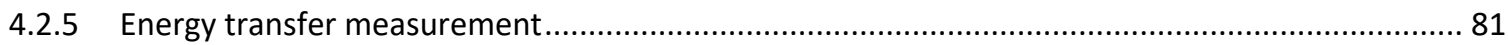

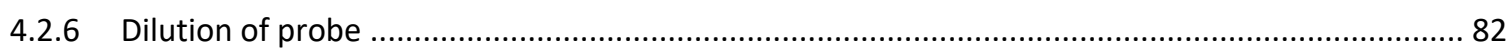

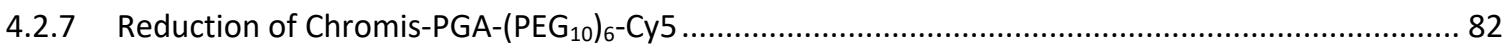

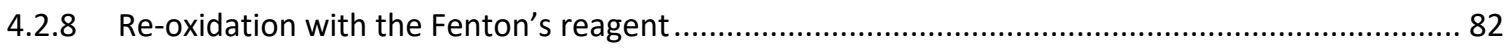

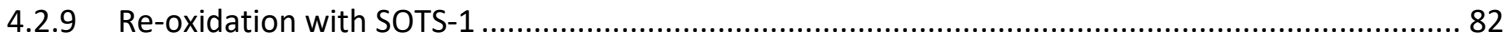

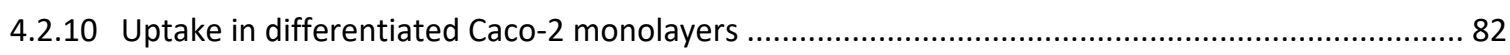

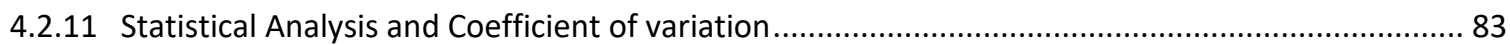

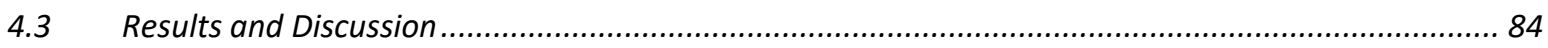

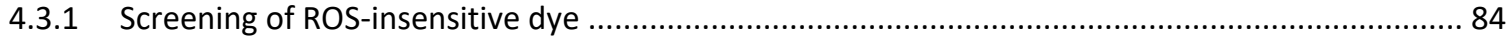

4.3.2 Synthesis, optimization of spacer length and characterization ................................................ 84

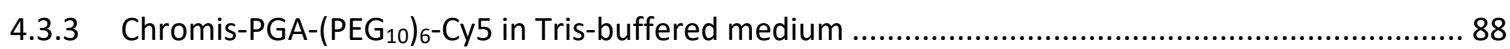

4.3.4 Uptake of Chromis-PGA-(PEG 10$)_{6}-\mathrm{Cy} 5$ in differentiated Caco-2 monolayers ................................8 88

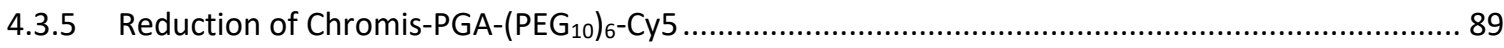

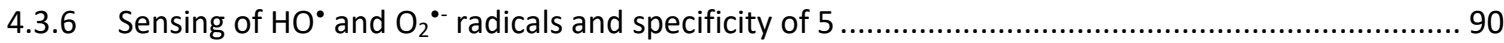

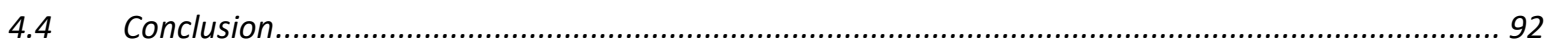




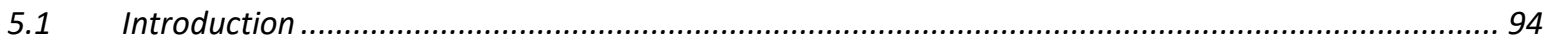

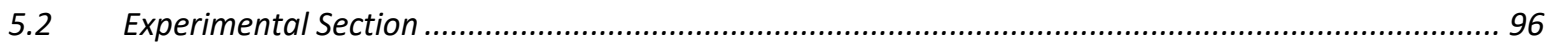

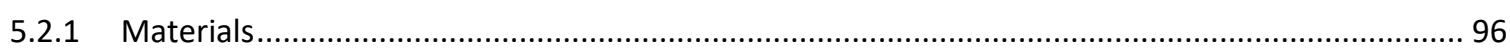

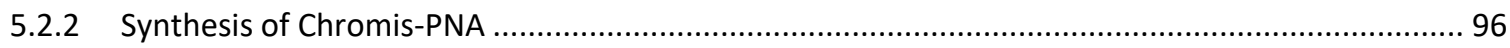

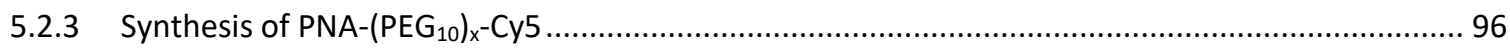

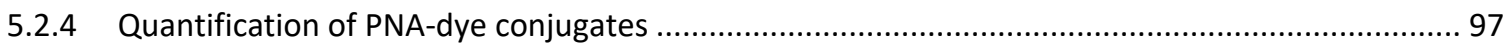

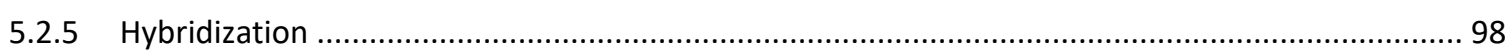

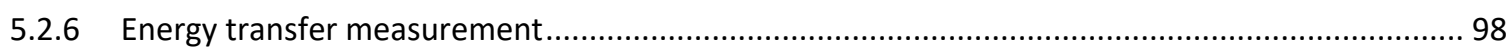

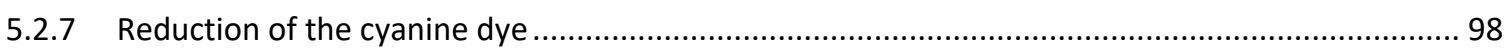

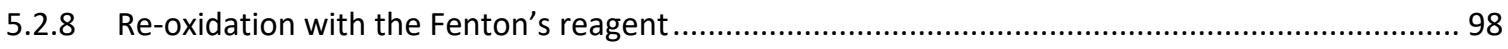

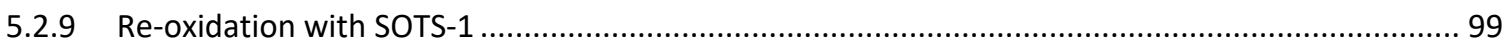

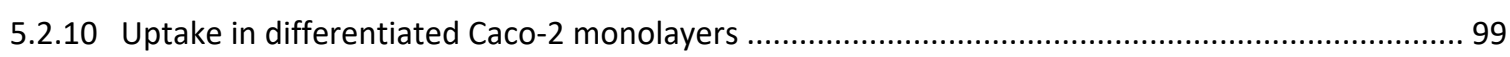

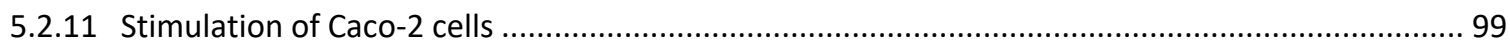

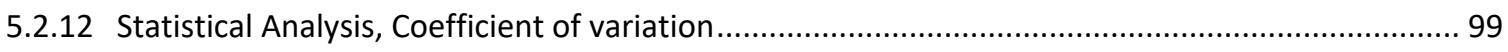

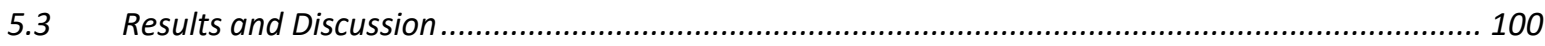

5.3.1 Synthesis and optimization of PNA-dye conjugates ........................................................... 100

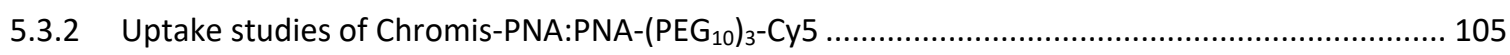

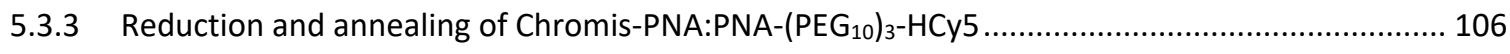

5.3.4 Sensing of $\mathrm{HO}^{\bullet}$ and $\mathrm{O}_{2}{ }^{\bullet-}$ radicals in cell free assays ......................................................... 106

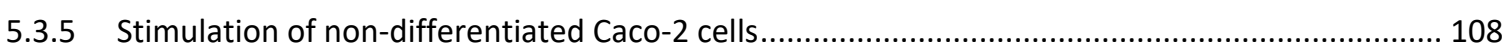

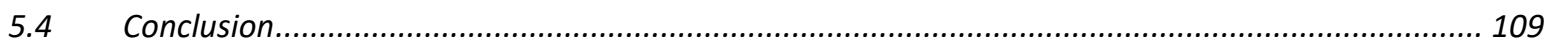

Chapter 6: Conclusion and Outlook.............................................................................................. 111

Appendix ............................................................................................................................................ 117

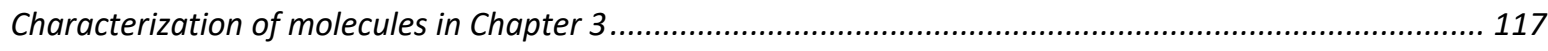

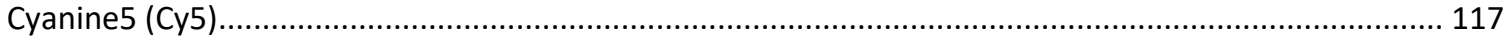

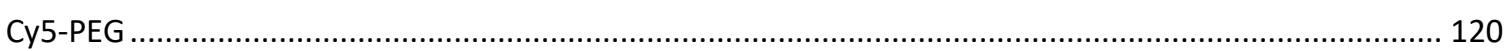

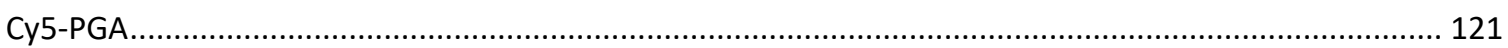

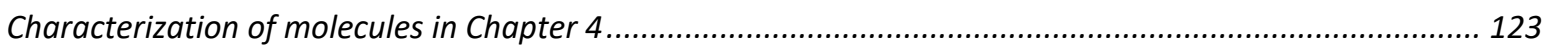

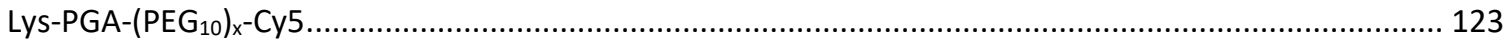

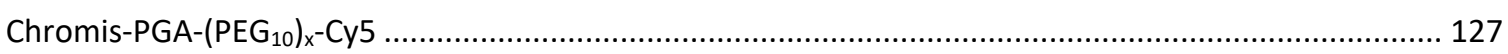

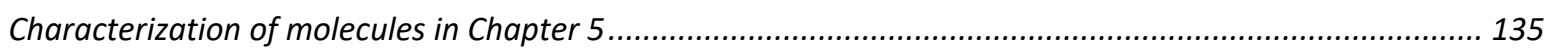

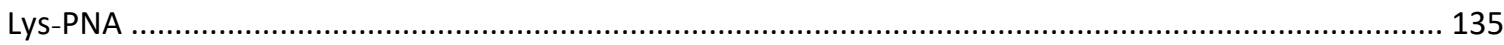

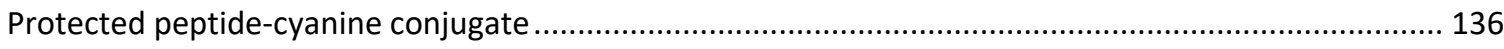

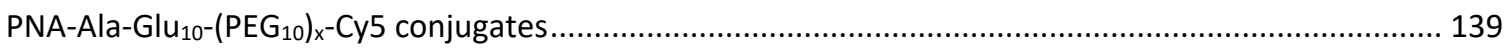




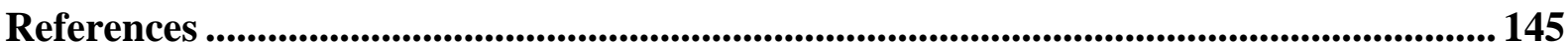

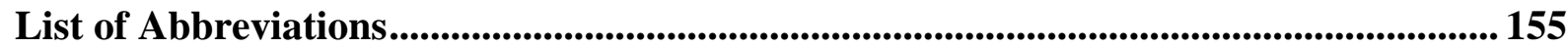

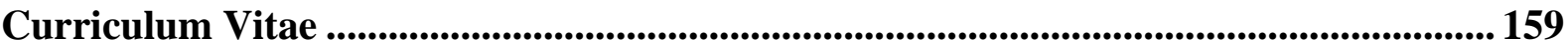

Scientific Contributions ................................................................................................. 161

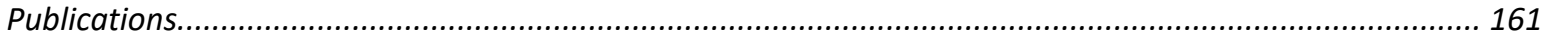

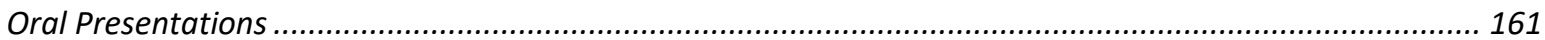

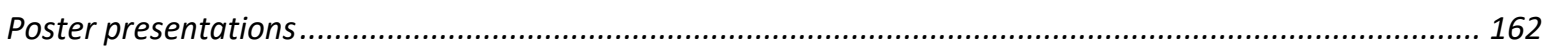

Acknowledgements....................................................................................................................................... 163 
Chapter 1

\section{Background and Purpose}


Traditionally, reactive oxygen species (ROS) were viewed as antimicrobial agents that were mainly produced during host defense mechanisms, such as the oxidative burst of neutrophils. ${ }^{[1-3]}$ The activity of ROS was long believed to be unspecific and their oxidative action towards lipids, amino acids and other biomolecules was accepted as a necessary evil. ${ }^{[4]}$ This perception has changed during the last two decades due to the discovery of various signaling functions of ROS. ${ }^{[5]}$ The ever widening spectrum now includes, but is not limited to, mediation of information during inflammatory responses, stem cell differentiation, cancer and ageing. ${ }^{[6-10]}$

In this work the term reactive oxygen species is used to describe oxygen containing reactive molecules. ${ }^{[1]}$ The primary species that is generated from molecular oxygen is its one-electron reduction product superoxide $\left(\mathrm{O}_{2}{ }^{--}\right)$, and is mainly produced by NADPH oxidases ${ }^{[12]}$ or through leakage in the electron transport chain in the mitochondria. ${ }^{[5]}$ Nitric oxide $\left(\mathrm{NO}^{\circ}\right)$ is the primary nitrogen containing ROS and is produced by NO synthases. ${ }^{[13]}$ Further downstream ROS are hydrogen peroxide $\left(\mathrm{H}_{2} \mathrm{O}_{2}\right)$, hypochlorous acid $(\mathrm{HOCl})$, hydroxyl radical $\left(\mathrm{HO}^{\circ}\right)$, singlet oxygen $\left({ }^{1} \mathrm{O}_{2}\right)$, ozone $\left(\mathrm{O}_{3}\right)$, various carbon peroxides and the nitrogen containing peroxynitrite $\left(\mathrm{ONOO}^{-}\right)$, nitroxyl (HNO) and nitrogen dioxide $\left(\mathrm{NO}_{2}\right){ }^{[10,14]} \mathrm{O}_{2}{ }^{--}$can either be converted into $\mathrm{H}_{2} \mathrm{O}_{2}$ by superoxide dismutase (SOD) or can react with nitric oxide (NO') to produce $\mathrm{ONOO}^{-} . \mathrm{H}_{2} \mathrm{O}_{2}$ is the precursor of $\mathrm{HOCl}$, which is generated by myeloperoxidase (MPO) and the $\mathrm{HO}^{\bullet}$ that is formed upon catalytic cleavage of the dioxygen bond by $\mathrm{Fe}^{2+}$ or $\mathrm{Cu}^{2+}$ (Figure 1-1). ${ }^{[15]} \mathrm{NO}^{\bullet}$ is a gaseous free radical that can recombine in a diffusion-controlled reaction with $\mathrm{O}_{2}{ }^{--}$, giving rise to $\mathrm{ONOO}^{-}$ or can be reduced and protonated leading to HNO (Figure 1-1). ${ }^{[16,17]}$ Distinguishing between the different ROS is a challenging task from a chemical point of view. Powerful analytical methods are therefore needed to elucidate the signal/stress dichotomy of these species.

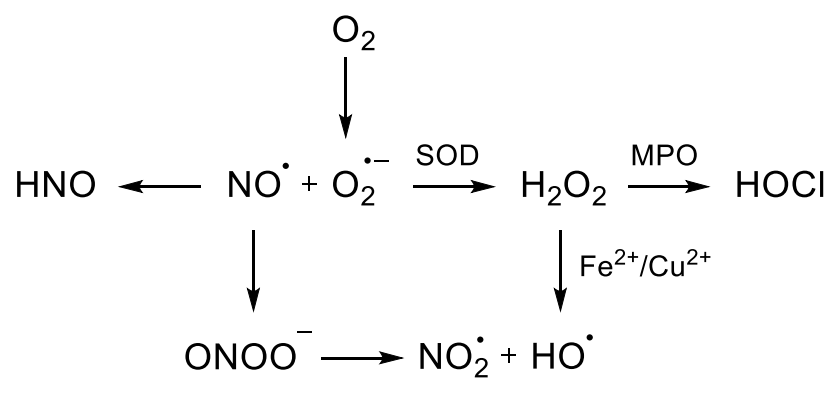

Figure 1-1: ROS generation in cells. 
Endogenous ROS can originate from different sites such as leakage from mitochondrial respiratory chain, xanthine oxidase, lipoxygenase, cytochrome $\mathrm{P} 450$ or by release of iron and copper ions from iron-sulphur clusters. However, NADPH oxidases (NOXs) are the only source that generates $\mathrm{O}_{2}{ }^{\circ-}$ not through leakage but on purpose. ${ }^{[18]} \mathrm{NOX}$ is a multicomponent transmembrane protein of which seven mammalian isoforms have been described, all sharing the feature of transferring electrons from NADPH across a membrane to reduce molecular oxygen to $\mathrm{O}_{2}{ }^{\cdot-}$ (Figure 1-2 a). ${ }^{[19,20]}$ NOX2 is the best studied homolog of this family and because it was first discovered in neutrophils and macrophages it is also called phagocyte NADPH oxidase. The required subunits to obtain a functional enzyme are a Rho guanosine triphosphatase ( $\operatorname{Rac} 1$ or $\operatorname{Rac} 2)$, the transmembrane proteins gp91 ${ }^{\text {phox }}$ and $\mathrm{p} 22^{\text {phox }}$, and the cytosolic components $\mathrm{p} 47^{\text {phox }}, \mathrm{p} 67^{\text {phox }}$ and $\mathrm{p} 40^{\text {phox }} \cdot{ }^{[21]}$ Electron transfer is ensured by a seven-step mechanism starting from cytosolic NADPH that donates two electrons to FAD, which is reduced to $\mathrm{FADH}_{2}$. By forming a semi-quinone, one electron is transferred to the inner heme, then to the outer heme and finally to molecular oxygen. The same cascade is repeated with the second electron from the FAD semi-quinone, reducing a second molecule of oxygen (Figure 1-2 a). Overall, this process is energetically favorable $\left(\Delta \mathrm{E}_{\mathrm{m}}=160\right.$ $\mathrm{mV}$ ), except for the step from the inner to the outer heme, which can only occur when oxygen is bound to the outer heme to accept the electron. ${ }^{[19]}$ The assembly of NADPH oxidase on the phagosomal and plasma membrane is triggered by the activation of neutrophils, which represents an important mechanism in host defense (Figure 1-2 c).

Phagocytic cells are a major endogenous source of ROS in the body, such as the neutrophils accounting for $70 \%$ of all white blood cells in circulation in humans. ${ }^{[1,18]}$ The migration of these polymorphonuclear leukocytes from the blood stream to the site of the microbial invasion is one of the first defense mechanisms during innate immune response. ${ }^{[22]}$ Upon reaching the infected site, neutrophils can harness a vast repertoire of antimicrobial weapons with the main objective of killing the intruders. Their diverse intra- and extracellular mechanisms include phagocytosis of the microorganism followed by antibacterial protein- and ROS-dependent killing (Figure 1-2 b (1)), degranulation of vesicles filled with antimicrobial agents (Figure 1-2 b (2)), and release of neutrophil extracellular traps (NETs) to immobilize and kill pathogens (Figure 1-2 b (3)). ${ }^{[23]}$ The generation of ROS, i.e. $\mathrm{O}_{2}{ }^{-}$, by neutrophils is called respiratory or oxidative burst and is accompanied by a suddenly increased $\mathrm{O}_{2}$ consumption due to NADPH oxidase activity. The generated $\mathrm{O}_{2}{ }^{--}$leads to downstream ROS as described in Figure 1-1, which may also have a higher 
a)

b)

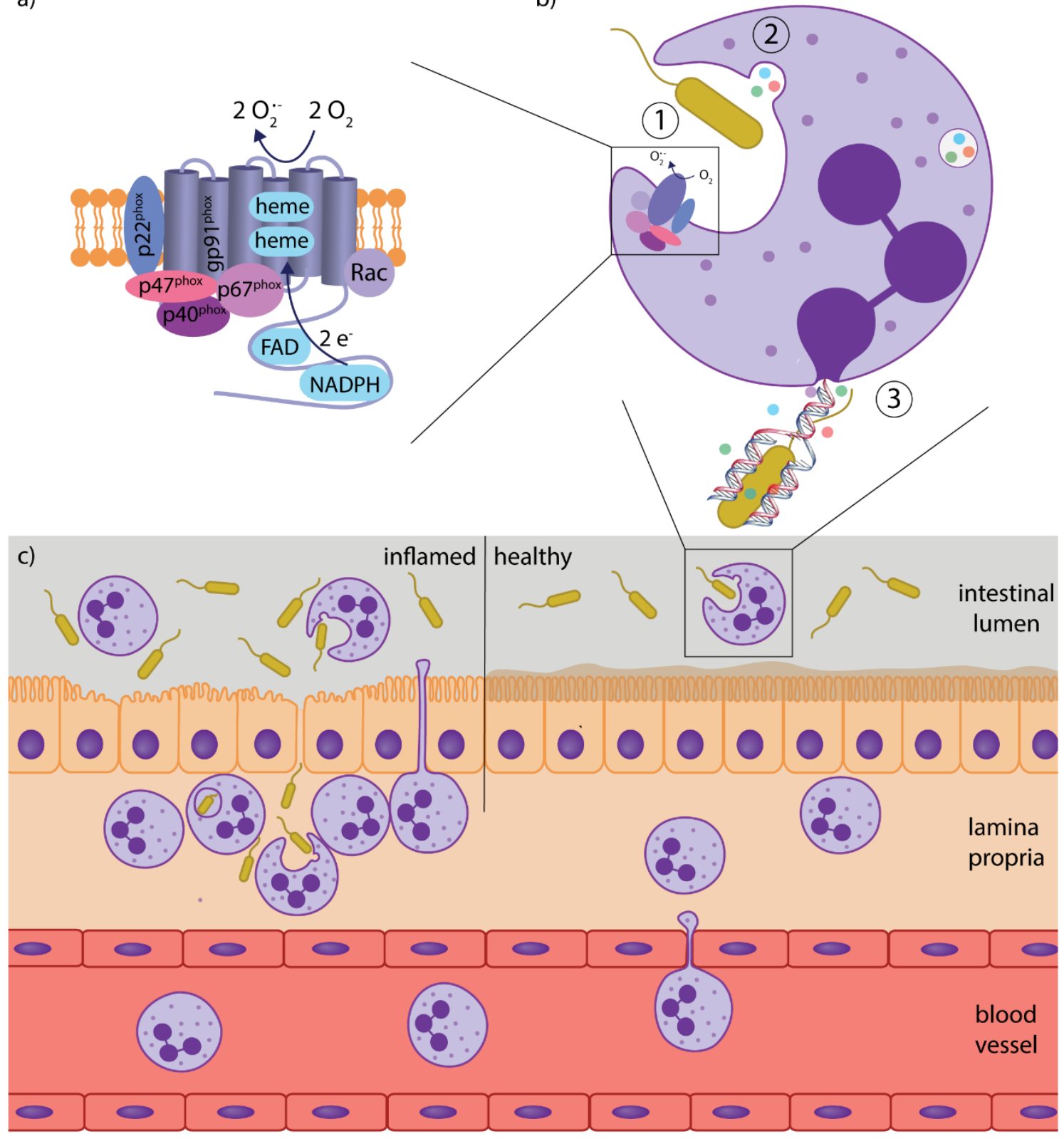

Figure 1-2: a) Active phagocytic NADPH oxidase, b) Neutrophil (1) phagocytosis and ROSdependent killing of microbes, (2) degranulation, (3) NETosis, c) neutrophil migration from blood to infected site in healthy and inflamed intestine.

antimicrobial activity than $\mathrm{O}_{2}{ }^{\circ-}$. Upon engulfment of the pathogens, ROS are generated in the phagosome and granules containing a plethora of peptides and proteins with detrimental effects on microbes fuse with the phagosomal membrane. ROS also functions as a second messenger during a process called lethal NETosis. During these events, neutrophils die upon releasing their complete DNA structures into the extracellular space, triggered by chromatin decondensation and spreading. Many bactericidal components of neutrophil granules adhere to these fibrous structures and make 
this active form of cell death a potent weapon to immobilize and kill especially large pathogens that are difficult to phagocytose. Additionally, two non-suicidal forms of NETosis have been discovered up to date and since in both cases the neutrophils remain alive, the processes have been referred to as vital NETosis (in contrast to lethal NETosis). The ROS-independent form of vital NETosis involves the release of DNA through nuclear decondensation but without excretion of the nuclear or plasma membrane. After these events, the neutrophils are still capable of engulfing pathogens and their lifespan is not affected by the loss of DNA. During the other variation of vital NETosis, only mitochondrial DNA is released and neutrophil nuclei are not involved in the process. ${ }^{[24,25]}$

The importance of NADPH oxidase-derived ROS becomes apparent in patients diagnosed with chronic granulomatous disease (CGD), a rare inherited disorder characterized by a (partly) dysfunctional NADPH oxidase. ${ }^{[26]}$ Young patients usually suffer from recurrent life-threatening bacterial and fungal infections due to defective neutrophils that cannot generate $\mathrm{O}_{2}{ }^{-}$nor downstream $\operatorname{ROS}\left(\right.$ e.g. $\mathrm{H}_{2} \mathrm{O}_{2}$ ). As a result, formation of granulomata and dysregulated inflammation in the genitourinary and gastrointestinal (GI) tracts are frequently observed. ${ }^{[27]}$ The manifestation of the disease in the GI tract is characterized by ulcers, abscesses, fistulae and obstructive symptoms. A similar pathological appearance is observed in certain cases of inflammatory bowel disease (IBD), which is a multifactorial inflammatory chronic disorder of the GI tract. IBD patients suffer from relapsing and chronic bowel inflammation and have a ten times greater risk of developing colorectal cancer. In IBD patients, neutrophils accumulate aberrantly within intestinal crypts during bowel inflammation and cause a prolonged inflammatory response. ${ }^{[28]}$ The very effective means of neutrophils to fight microbes backfire in these situations, and lead to an excessive recruitment of inflammatory cells and tissue damage (Figure 1-2 c). The abnormal accumulation of neutrophils observed in IBD leads to a tremendously elevated ROS generation and results in the oxidation of DNA, proteins, lipids and other biomolecules.

The role of ROS production and its influence on the course of disease is still not unequivocally established. Hence, tools with high spatio-temporal resolution to specifically detect ROS at the site of inflammation would provide more evidence to this debate and would be of great value. Fluorescent probes represent a unique mean to detect changes in analyte concentration in real-time using inexpensive equipment. ${ }^{[29,30]}$ Stimuli-responsive switch-on sensors can be used to enhance the signal-to-noise ratio but also to ensure specific detection of different ROS. ${ }^{[31-33]}$ Their general 
drawback is an inherent concentration dependence, that can be overcome by monitoring the change of ratio of two fluorescence emissions. ${ }^{[34-36]}$ This type of sensors are referred to as ratiometric probes and a summary of such indicators for ROS is provided in Chapter 2, followed by a critical discussion of selected examples. As it was shown that extracellular ROS have a distinct function in inflammation, ${ }^{[37]}$ wound healing, ${ }^{[38]}$ and are important regulators in ROS-mediated apoptosis in tumor cells, ${ }^{[39]}$ discrimination between extra- and intracellular ROS is pivotal to better understand their complex and diverse functions. Most fluorescent ROS sensors are designed to detect only intracellular analytes. With the goal of expanding the detection possibilities to the extracelluar space, we designed an extracellular ratiometric probe to allow real-time imaging of ROS. First, the intracellular hydrocyanines introduced by Kundu et al. ${ }^{[40]}$ were evaluated and compared to other commercially available ROS sensors, as reported in Chapter 3. Then, cell-free assays as well as cell-based models were established and used to show that ROS detection with hydrocyanines was possible. Additionally, a model fluorophore was selected for our study and was successfully modified to reduce internalization by cells. The sensor was made ratiometric by linking a stably emitting reference fluorophore to the ROS sensor (Figure 1-3 a). The spacer consisted of negatively charged glutamic acids and poly(ethylene glycol) (PEG) units to connect the two dyes yet keeping them spatially apart. The linker also increased the probe's solubility and reduced its cellular uptake. The synthesis and characterization of the sensor are described in Chapter 4, together with a discussion of its validation in sensing assays. To further improve our system, a peptide-based DNA mimic (peptide nucleic acid, PNA) was introduced in the linker, which allowed handling the two fluorophores separately and increased the modularity and versatility of the sensor design (Figure 1-3 b). The design, synthesis and validation of the flexible platform are outlined in Chapter 5. Overall, a prototype of a modular ratiometric probe was designed and synthesized. Our findings, summarized in Chapter 6 , suggest that extracellular $\mathrm{O}_{2}{ }^{\circ-}$ detection is possible with hydrocyanines using our ratiometric probes, but that their sensitivity is greatly decreased when coupled to a macromolecule. Generally, a strong influence of the medium was observed, which may complicate interpretation of cell and in vivo studies. 
a)

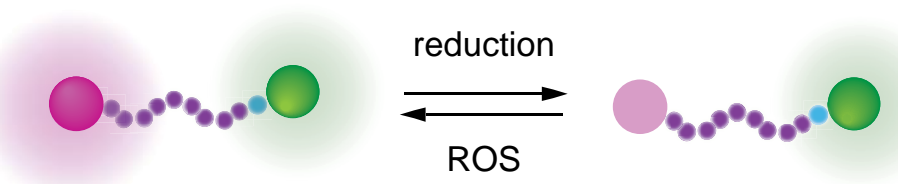

b)
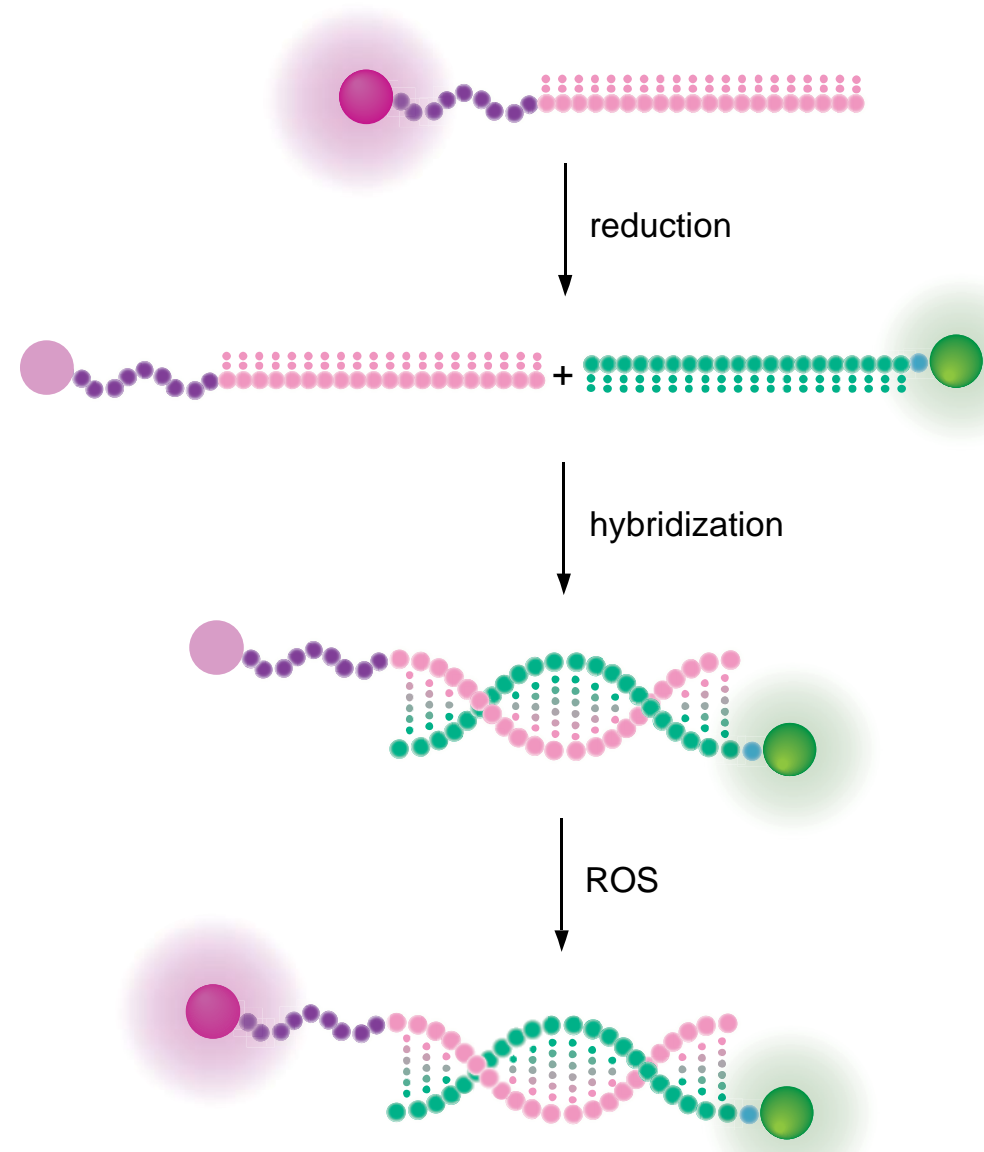

Figure 1-3: General design of the ratiometric sensors. a) Schematic representation of a ROSsensor that is covalently linked to the reporter dye discussed in Chapter 4; b) Schematic representation of the modular approach using PNA to combine the building blocks outlined in Chapter 5. 


\section{Chapter 2}

\section{Ratiometric Fluorescent Probes to Detect Reactive Oxygen Species}

This chapter is accepted for publication in:

D. Andina, J.-C. Leroux, P. Luciani, Ratiometric Fluorescent Probes to Detect Reactive Oxygen and Nitrogen Species, Chem. - A Eur. J. doi:10.1002/chem.201702458 


\subsection{Introduction}

Common means to study ROS include the analysis of specific biomolecule or lipid oxidations by mass spectrometry, the use of spin trapping techniques, as well as colorimetry-, chemiluminescence- and fluorescence-based imaging approaches ${ }^{[41-45]}$ Fluorimetry is especially suitable for applications in cell culture and in vivo, as it allows real-time monitoring of the changes in analyte concentration in a non-invasive and relatively inexpensive way. ${ }^{[30]}$ The most widely used fluorophores (2',7'-dichlorofluorescein diacetate (DCFHDA), dihydrorhodamine 123 (DHR), hydroethidine (HE or DHE), hydroxyphenyl fluorescein (HPF)) react directly with ROS, switching from a non-fluorescent to a fluorescent state. Despite being routinely used to detect, and sometimes even quantify, specific ROS, these dyes react in fact with multiple ROS. Additionally, their turnon mechanism is radical-mediated, which can lead to numerous side reactions and selfamplification of the fluorescence signal. Therefore, these probes allow the qualitative evaluation of the general redox-state of a sample rather than the quantitative monitoring of a specific analyte ${ }^{[46,47]}$ For a more detailed discussion of the limitations of traditional probes and their applications in biological systems, the reader is referred to a review by C.C. Winterbourn. ${ }^{[48]}$

With the aim of ameliorating ROS detection, new generations of fluorescent probes have been proposed in recent years. ${ }^{[33,49,50]}$ Various fluorophore scaffolds (e.g. rhodamine, coumarin, BODIPY, cyanine dyes) have been modified to obtain new sensors with different fluorescence excitation/emission wavelengths and physico-chemical properties. ${ }^{[34,51]}$ The turn-on response has also been progressively adjusted by fine-tuning the electronic properties of the analyte-reactive site, yielding more sensitive and specific sensors. ${ }^{[36]}$ Concurrently, nanoparticles and polymers have been introduced as ROS sensors, relying on their direct interaction with reactive species or their combination with small organic fluorophores. ${ }^{[52-55]}$ In general, the cytotoxicity of these emerging sensors - and especially of those based on polymers and nanoparticles - has been investigated and reported in the literature. In addition, probes equipped with organelle-targeting units, such as the mitochondrial targeting moiety triphenyl phosphine (TPP), have been implemented to provide spatially confined information on ROS production. ${ }^{[56-58]}$

Newly developed sensors are primarily characterized by measuring their sensitivity and specificity in cell-free media. However, due to the intrinsic challenges in detecting ROS in biological systems, the promising properties observed in cell-free assays do not necessarily translate successfully in more complex in vitro or in vivo set-ups. Hence, sensor validation should be carried out in the 
tissue culture setups and/or animal models for which they have been devised in order to more accurately estimate their potential in biological applications. ${ }^{[48]}$ In cell-based assays, cells are usually challenged with stimulants to induce a stress response with concomitant ROS production, i.e. the stimulation of murine macrophages RAW 264.7 by phorbol-12-myristate-13-acetate (PMA). In animal models, a stress response can also be induced by stimulants (e.g. lipopolysaccharides (LPS)) or by a genetically modified phenotype that results in altered ROS levels. Although in vivo monitoring would allow for the most complete evaluation of a sensor's potential, technical limitations are likely to arise from the short tissue penetration depth and the high tissue auto-fluorescence encountered at wavelengths below $650 \mathrm{~nm}$. Thus, molecules emitting in the near infrared (NIR) region are generally preferred to enable more reliable analyte detection during in vivo sensing. ${ }^{[59]}$ Another relevant potential limitation when imaging biological systems is the fluctuation of fluorescence emission intensity stemming from concentration differences of the sensor, which can lead to flawed results. ${ }^{[30]}$ In ratiometric analyte detection this issue is overcome by establishing a ratio between two wavelengths (excitation or emission) thereby creating a signal read-out that is sensor concentration independent. A deceptive simple way to introduce a second fluorescence emission consists of adding a ROS-insensitive dye to a ROSsensor, thereby obtaining a physical mixture of two fluorophores. Unequal tissue distributions of the two dyes can, however, lead to inaccurate data acquisition and, therefore, it should not be used for ratiometric sensing. ${ }^{[60]}$ Another possible strategy is the development of a ratiometric sensor from an intensity-based precursor. By coupling the sensor to a polymer or nanoparticle that either functions as anchor or also exhibits a reference emission, a ratiometric probe can be created. Alternatively, a shift in fluorescence emission wavelength can be detected. A frequently applied strategy is based on the Förster resonance energy transfer (FRET) effect, in which energy is transferred from a donor to an acceptor dye leading to a shift of fluorescence emission intensities. These probes consist of a dyad of two fluorophores or a fluorescent polymer/nanoparticle and a fluorophore, respectively. The reaction of an intensity-based sensor with the analyte of interest either enables or disables FRET leading to a shift of emission intensities. Alternatively, single small organic molecules may be used as ratiometric indicators in the cases where a shift in emission wavelength is detected upon reaction with the analyte through an internal charge transfer (ICT) mechanism. ${ }^{[61,62]}$ 
Sensing of ROS has been achieved with ratiometric probes through very diverse approaches, each of them offering advantages as well as limitations depending on the intended application. In this chapter, we present a comprehensive summary of the advances made in the field, giving an overview of current ratiometric fluorescent probes for ROS available in literature and critically discussing selected approaches, with a special focus on their application in cell cultures and in vivo systems.

A class of fluorescent probes that will not be discussed in this review are the genetically encoded fluorescent sensors. These protein-based probes exhibit a change in ratiometric emission signal due to small conformational changes of the proteins as a response to their redox environment. As the conformational changes are non-permanent, reversible monitoring is possible. In addition, these probes are photostable, biocompatible and targeting of subcellular compartments was achieved. For a more detailed description on this class of probes, the reader is referred to other reviews on basic concepts and recent advances in the field. ${ }^{[63-66]}$ 


\subsection{Ratiometric fluorescent probes to detect ROS}

\subsubsection{Probes for $\mathrm{H}_{2} \mathrm{O}_{2}$}

$\mathrm{H}_{2} \mathrm{O}_{2}$ is one of the most studied ROS and is known to be involved in mechanisms regulating health, ageing and disease. Compared to other reactive species, this two-electron oxidant reacts slowly with biological molecules (e.g., with glutathione (GSH) $\mathrm{k}=0.9 \mathrm{M}^{-1} \mathrm{~s}^{-1}$ ) and has an approximate diffusion distance of $1.5 \mathrm{~mm}$ in the presence of cellular GSH concentrations $(2 \mathrm{mM}) .{ }^{[15]}$ Despite its intrinsic limitations (vide supra), DCFHDA has often been used to study $\mathrm{H}_{2} \mathrm{O}_{2}$. However, recurrent reports on its alleged unspecificity, over-oxidation and spontaneous auto-oxidation ${ }^{[48]}$ led to the introduction of novel sensors based on alternative detection strategies, such as the chemoselective oxidation of boronates. In this highly successful approach, a boronate ester is implemented in the structure of a dye to quench or alter its fluorescence emission after which $\mathrm{H}_{2} \mathrm{O}_{2}$ -but also other ROS- react with the boronate group and trigger a fluorescence turn-on response (Figure 2-1). Chang and coworkers synthesized and systematically studied boronate-based sensors, and applied many of them to biological systems. ${ }^{[67]}$ Their seminal work led to the widespread application of this new class of indicators, as confirmed by the use of boronate oxidations in all the ratiometric $\mathrm{H}_{2} \mathrm{O}_{2}$-sensing probes published to date with only one very recent exception (Table 2-1). An early development by Chang's research group was based on a 1,8-naphthalimide derivative (PL1) that shifted from $475 \mathrm{~nm}$ to $540 \mathrm{~nm}$ upon arylboronate oxidation, showing a 12fold increase in ratiometric signal $\left(I_{540} / I_{475}\right){ }^{[68]}$ Wen et al. used the same dye scaffold, but modified the $\mathrm{H}_{2} \mathrm{O}_{2}$ recognition site to a pinacol boronate ester (NP1), which resulted in a 20-times higher fluorescence ratio increase at the same $\mathrm{H}_{2} \mathrm{O}_{2}$ concentration after $1 \mathrm{~h}$. Notably, even though a more pronounced change in signal ratio was observed for NP1 in cell-free assays, its response in PMAstimulated RAW 264.7 cells was similar to the one of PL1 (1.3 vs. 1.5-fold increase), demonstrating that the direct translation of a sensor's potential cannot be assumed from in vitro to living systems. ${ }^{[69]}$ Furthermore, the amino substituent of NP1 contained an additional azide to

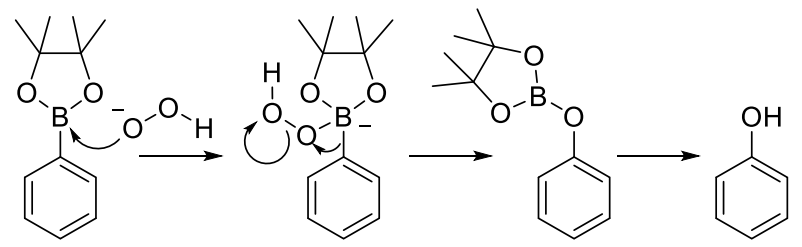

Figure 2-1: Mechanism of $\mathrm{H}_{2} \mathrm{O}_{2}$ with boronate ester. Adapted from ${ }^{[67]}$. 
enable the coupling via click chemistry of peptides targeting specific subcellular compartments. Confocal laser scanning microscopy revealed that the fluorophore lacking targeting moieties (NP1) predominantly resided in the cytoplasm, whereas Pep-NP1 and Pep3-NP1 accumulated in the nucleus and the mitochondria respectively, due to their targeting peptides Pep and Pep3. ${ }^{[69,70]}$ NP1 was further tested ex vivo on hippocampal cuts, on which it yielded a 1.5-fold increase after $\mathrm{H}_{2} \mathrm{O}_{2}$ addition. Even though emission intensity fluctuations can be minimized with ratiometric probes, high background noise due to tissue auto-fluorescence can complicate signal read-outs, especially for the blue and green lasers used with most of these probes. High-energy lasers can also damage the sample or induce a stress response, which is why NIR or two-photon dyes that can be excited with red lasers are more suitable for in vivo as well as ex vivo studies. ${ }^{[51]}$ Two ratiometric approaches have been designed using existing two-photon indicators equipped with an arylboronate ester as sensing unit. In comparison to the one-photon sensors PL1 and NP1, the turnon response of PN1 and SHP-Mito in PMA-stimulated RAW 264.7 cells proved to be superior, and the pulsed $750 \mathrm{~nm}$ laser used allowed imaging in biological samples with a greatly reduced tissue auto-fluorescence. In principle, the lower background signal achieved with two-photon indicators should simplify the detection of $\mathrm{H}_{2} \mathrm{O}_{2}$ in hippocampal cuts. For PN1, however, this did not translate into a higher increase in ratiometric signal (1.2 to 1.6-fold) compared to the onephoton probes. A proof of concept was achieved with the mitochondrial targeting probe SHP-Mito that showed a 2.2-fold increase in ratiometric signal. ${ }^{[71,72]}$ In another targeted approach, Wu and coworkers proposed an elegant method to convey a fluorescein-based sensing unit to the mitochondria by means of a TPP moiety covalently linked to an amino-coated carbon dot. Although a modest 4.4-fold increase in the emission ratio (I550/I457) was achieved in cell-free assays, a striking 50-fold increase in fluorescence ratio was observed in PMA-stimulated RAW 264.7 cells. $^{\text {[73] }}$ However, the reaction time of the nanoprobe with $\mathrm{H}_{2} \mathrm{O}_{2}$ was slow (>35 min), making it unsuitable for in vivo imaging. The same group proposed a similar system in which a faster responding sensing unit (3 min) was anchored to a carbon dot, resulting in a 34-fold change in the ratiometric signal in $20 \mathrm{mM}$ HEPES buffer. Ratiometric imaging with the nanoprobe was demonstrated in biological systems through addition of $\mathrm{H}_{2} \mathrm{O}_{2}$ to L929 cells, as well as in five-day old zebrafish larvae, where it successfully detected doxorubicin-induced oxidative damage in the gut region (Figure 2-2). ${ }^{\text {[74] }}$ 
DoX

incubation

$\mathrm{Oh}$

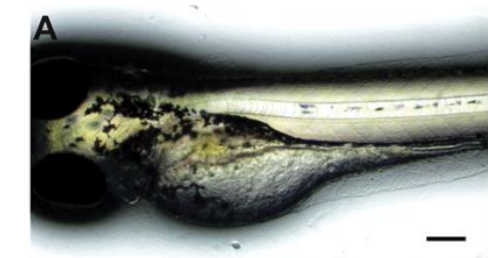

$24 \mathrm{~h}$

$48 \mathrm{~h}$
Fluorescence
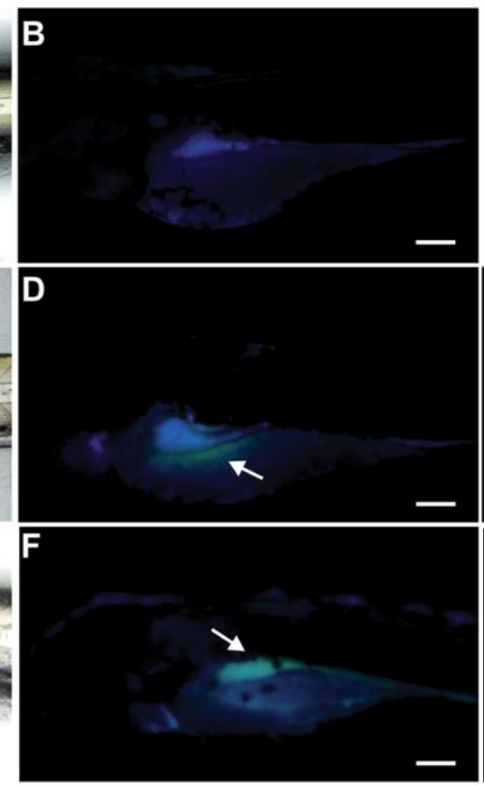

\section{Pseudo-color}

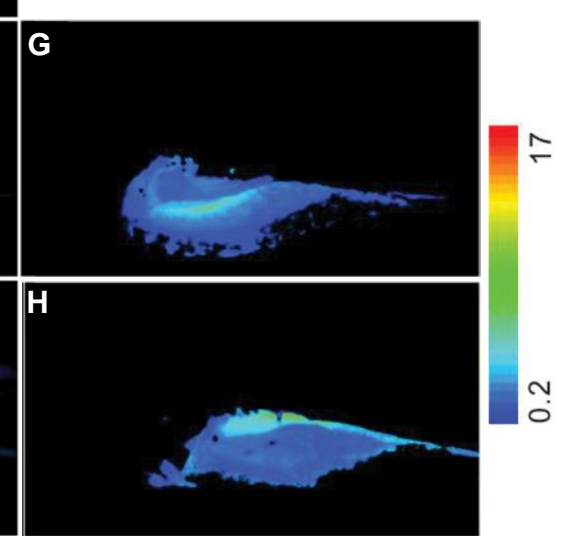

Figure 2-2: Microscopy images of five-day old zebrafish larvae. (A) Brightfield and (B) fluorescence images of a zebrafish incubated with $0.1 \mathrm{mg} \mathrm{mL}-1$ nanoprobe for $60 \mathrm{~min}$. (C) Brightfield and (D) fluorescence images of a zebrafish incubated with DOX for $24 \mathrm{~h}$ and then with $0.1 \mathrm{mg} \mathrm{mL} \mathrm{m}^{-1}$ nanoprobe for $60 \mathrm{~min}$. (E), (F) Brightfield and fluorescence images of a zebrafish incubated with DOX for $48 \mathrm{~h}$ and then incubated with the $0.1 \mathrm{mg} \mathrm{mL}^{-1}$ nanoprobe for $60 \mathrm{~min}$. The white arrows indicate the location of the strongest ratiometric response due to endogenously generated $\mathrm{H}_{2} \mathrm{O}_{2}$. $(\mathrm{G})$, (H) Fluorescence microscopy images showing the ratio of fluorescence emission (Igreen/Iblue) in pseudo-color. Scale bar: $200 \mathrm{~mm}$. Adapted from $\mathrm{Wu}$ et al. with permission. ${ }^{[74]}$

These promising examples illustrate the continuous progress made in the design of ratiometric probes to detect $\mathrm{H}_{2} \mathrm{O}_{2}$, which has so far enabled remarkable achievements such as the targeting of specific intracellular compartments, and the in vivo application of a few sensors. While boronatebased probes may be widely applicable tools for $\mathrm{H}_{2} \mathrm{O}_{2}$ sensing, results should nevertheless be interpreted with caution. Kinetic studies have revealed that boronate oxidation is several orders of magnitude faster with $\mathrm{HOCl}\left(\mathrm{k}=6.2 \times 10^{3} \mathrm{M}^{-1} \mathrm{~s}^{-1}\right)$ and $\mathrm{ONOO}^{-}\left(k=10^{6} \mathrm{M}^{-1} \mathrm{~s}^{-1}\right)$ than with $\mathrm{H}_{2} \mathrm{O}_{2}(k$ $\left.=2.2 \mathrm{M}^{-1} \mathrm{~s}^{-1}\right) .{ }^{[75]} \mathrm{ONOO}^{-}$has not been tested as alternative oxidant in early approaches, and whereas the interference of $\mathrm{HOCl}$ has been investigated more frequently, this was generally done in HEPES buffer which is known to scavenge $\mathrm{HOCl}$. Furthermore, sensitivity and selectivity evaluations in cell-free assays do not accurately reflect a probe's in vitro and/or in vivo detection power, especially when the studies have been carried out in solvent/buffer mixtures due to solubility issues of organic molecules. In general, the sensitivity and selectivity of a newly developed sensor should be tested in media that mimic biological systems more closely (e.g. addition of serum). Further biological 
studies - carried out in various research laboratories and with different disease models - would also be needed to validate the general potential of these new probes. 
Table 2-1: Ratiometric fluorescent probes for the specific detection of $\mathrm{H}_{2} \mathrm{O}_{2}$.

\begin{tabular}{|c|c|c|c|c|c|c|}
\hline \multirow[t]{2}{*}{ Structure } & \multirow[t]{2}{*}{ Name } & \multirow{2}{*}{$\begin{array}{l}\text { Emission } \\
\text { ratio }\end{array}$} & \multicolumn{2}{|c|}{$\begin{array}{c}\text { Maximum increase in } \\
\text { emission ratio measured }\end{array}$} & \multirow{2}{*}{$\begin{array}{c}\text { Biological system / } \\
\text { stimulant }^{\mathrm{c}}\end{array}$} & \multirow[t]{2}{*}{ Ref. } \\
\hline & & & cell-free & biological & & \\
\hline \multicolumn{7}{|c|}{$\mathrm{H}_{2} \mathrm{O}_{2}-$ small molecules } \\
\hline & RPF1 & $517 / 464$ & 8-fold & n.a. & n.a. & [76] \\
\hline & PL1 & $540 / 475$ & 12-fold & 1.5-fold & RAW 264.7 / PMA and $\mathrm{H}_{2} \mathrm{O}_{2}$ & [68] \\
\hline & & & & 2.3-fold & RAW 264.7 / PMA, $\mathrm{H}_{2} \mathrm{O}_{2}$ & \\
\hline & & & & 1.2-1.6-fold & Hippocampal cuts $/ \mathrm{H}_{2} \mathrm{O}_{2}$ & \\
\hline & AC-bor & $484 / 566$ & 9.5-fold & qualitative & $\mathrm{PC} 3 / \mathrm{H}_{2} \mathrm{O}_{2}$ & [77] \\
\hline
\end{tabular}




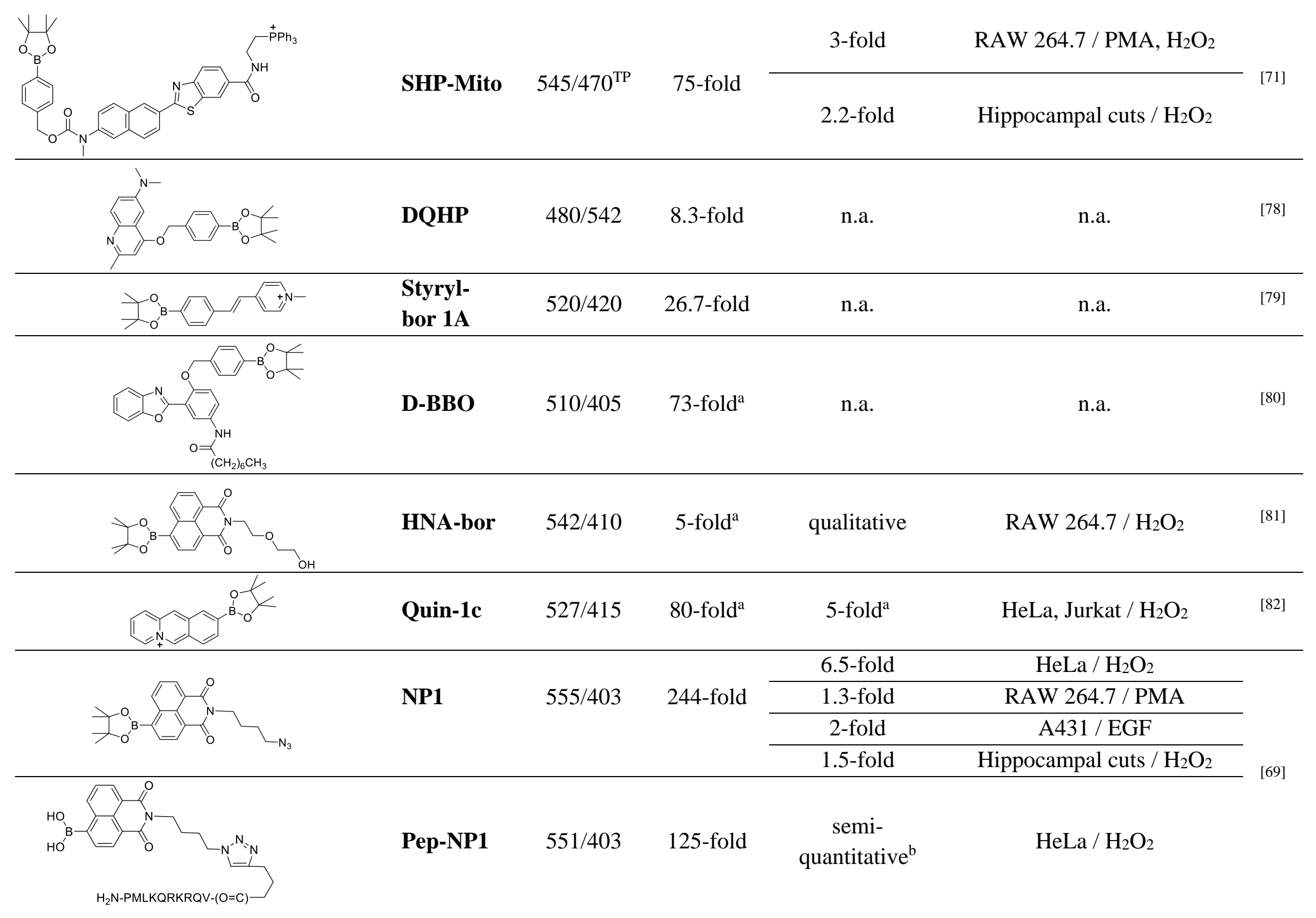




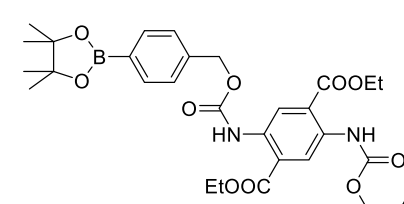

DAT-B $\quad 574 / 500 \quad 8.3-$ fold $^{\mathrm{a}} \quad$ n.a

n.a.

[83]

Etooc

DAT-B $-1.3-\mathrm{fold}$

(1)

\section{(n)}

Pep3-Np1 555/646 12-fold 1.5-fold $\quad \mathrm{HeLa} / \mathrm{H}_{2} \mathrm{O}_{2}$

[70]

$\mathrm{H}_{2} \mathrm{~N}-(\mathrm{WTK}) \mathrm{KK}_{\mathrm{N} \varepsilon} \mathrm{TW}-(\mathrm{O}=\mathrm{C})$

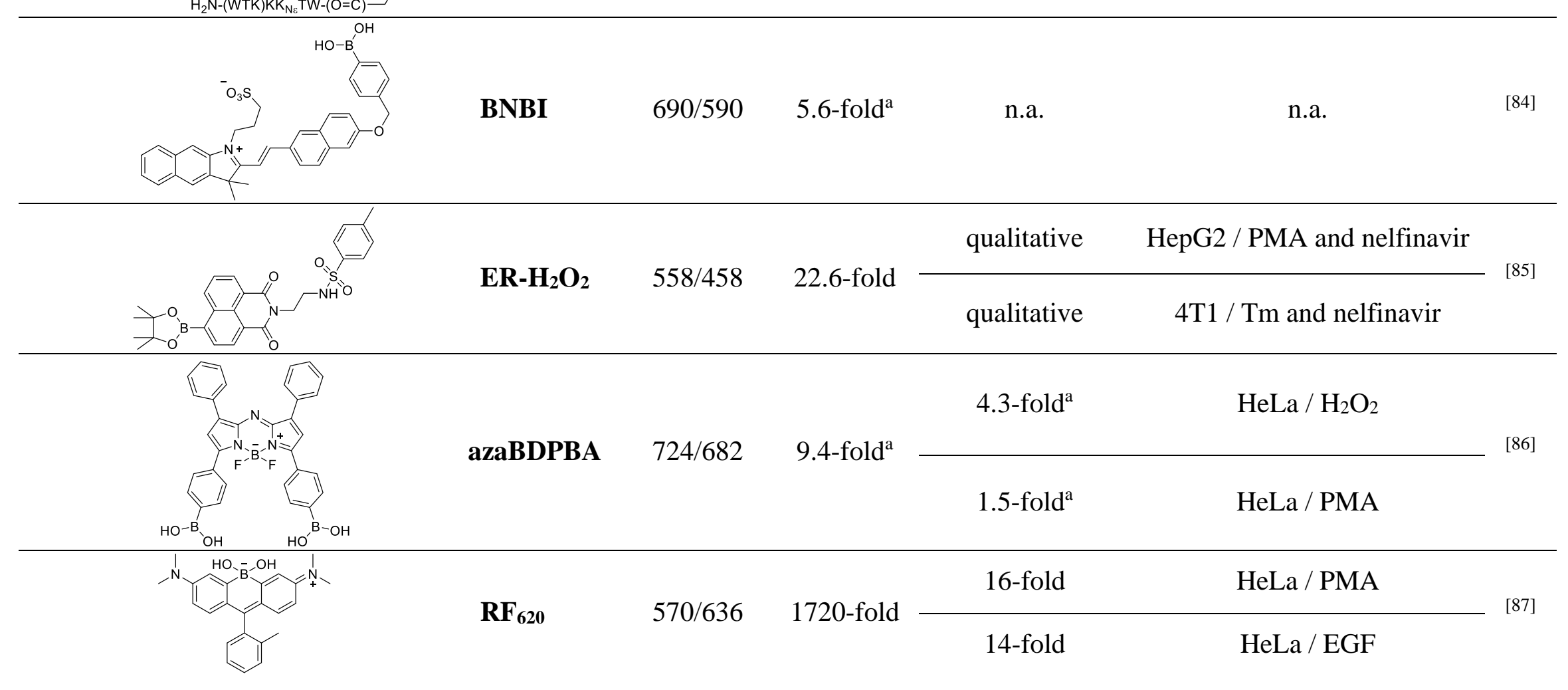




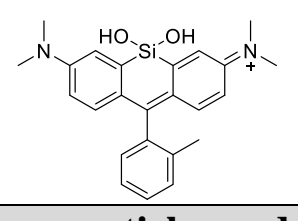
SiOH2R
$570 / 681$
11-fold
4.26-fold
HeLa / PMA

\section{$\mathrm{H}_{2} \mathrm{O}_{2}$ - nanoparticles and polymers}

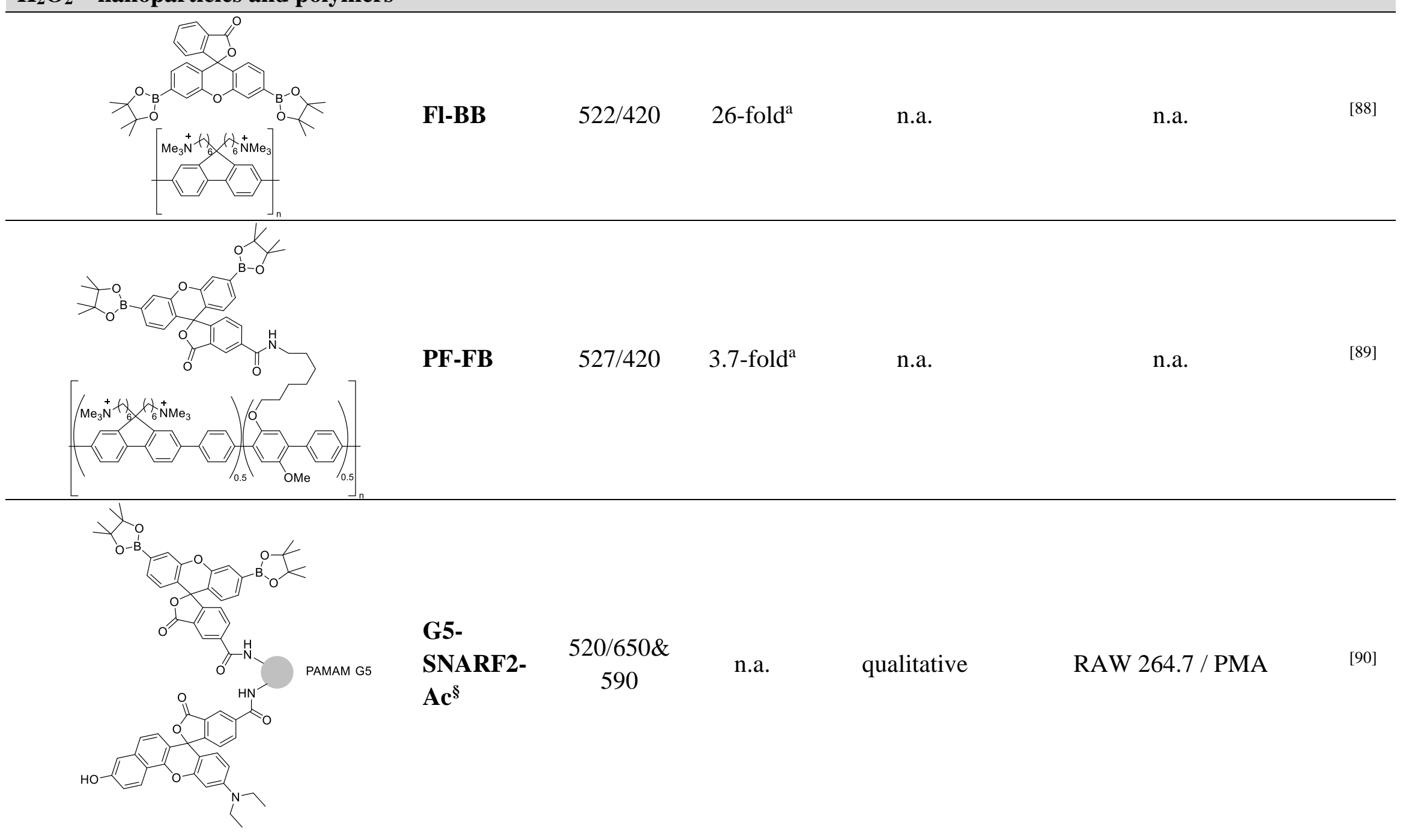




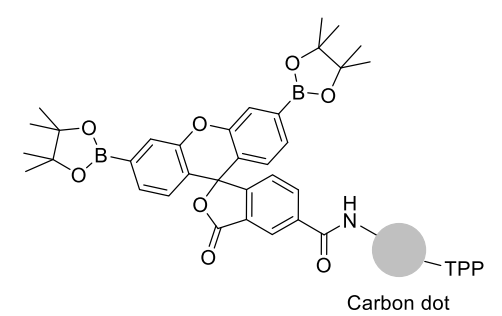

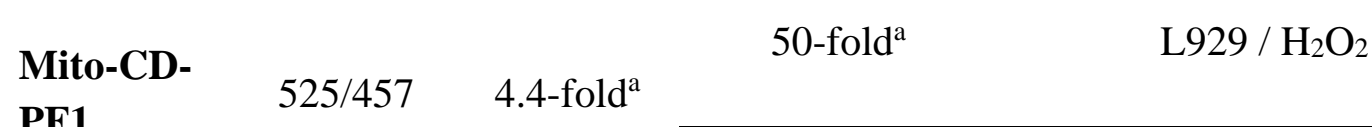

Carbon dot

71-fold ${ }^{\mathrm{a}}$

RAW 264.7 / PMA

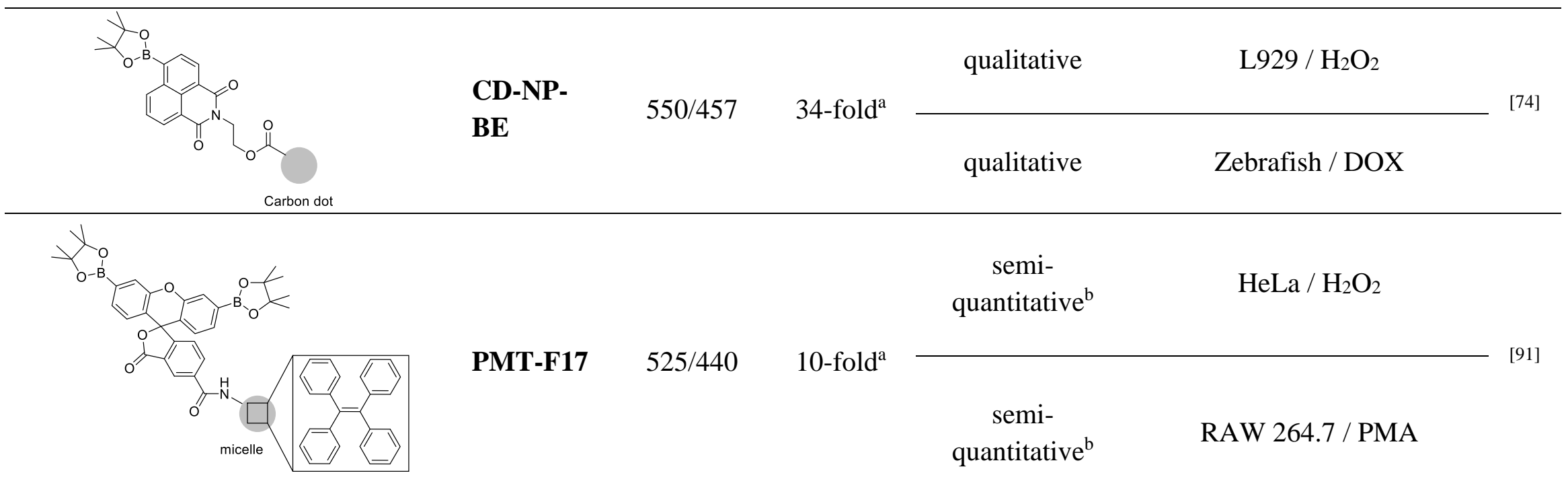

${ }^{\mathrm{a}}$ : values calculated from graphs with plot digitizer, ${ }^{\mathrm{b}}$ : only color scale bar available ${ }^{\mathrm{c}}$ : compound used to induce or mimic stress response $^{\text {TP}}$ : two-photon probe, PMA: phorbol-12-myristate-13-acetate, DOX: doxorubicin, Tm: tunicamycin, EGF: epidermal growth factor, ${ }^{\S}$ dual responsive probe to $\mathrm{H}_{2} \mathrm{O}_{2}$ and $\mathrm{pH}$, n.a.: not available. 


\subsubsection{Probes for $\mathrm{HOCl}$}

$\mathrm{HOCl}$, commonly known as bleach, is frequently used as antimicrobial agent in households. In the body, $\mathrm{HOCl}$ is produced by myeloperoxidase from $\mathrm{H}_{2} \mathrm{O}_{2}$ and $\mathrm{Cl}^{-}$, and its antimicrobial activity plays an important role during innate immune response. Its effects arise from diverse oxidation and chlorination reactions with biomolecules such as lipids, proteins or nucleic acids. ${ }^{[6]}$ With the aim of exploiting this oxidative power, various fluorescent sensors for $\mathrm{HOCl}$ have been proposed (Table 2-2). For instance, the spirolactam opening of rhodamine has been applied in $\mathrm{HOCl}$ and $\mathrm{NO}^{\bullet}$ detection after having found wide-spread use in many metal sensing approaches. In Figure 23, the two main mechanisms for $\mathrm{HOCl}$ sensing are depicted. In one of them, the selective oxidation of a hydrazo group is exploited with subsequent release of the fluorophore by hydrolysis (Figure 2-3 a). In the other approach, a thiohydrazide is used instead, resulting in an irreversible oxadiazole formation (Figure 2-3 b). ${ }^{[92]}$ The spirolactam ring-opening was exploited in combination with a FRET mechanism in several ratiometric probes. ${ }^{[93-104]}$ Rhod-TMC, Cou-Rho and RMCIO-2, for example, used coumarin as donor dye and employed a rigid six-membered ring to connect it to the acceptor fluorophore, i.e. the rhodamine-based senor. ${ }^{[93,95,96]}$ Whereas a thiohydrazide was used in Rhod-TMC, the selective oxidation of the hydrazo group was exploited in Cou-Rho and RMCIO2 using the same coumarin-rhodamine dyad with different leaving groups. In terms of ratiometric signal, Cou-Rho showed a lower increase in cell-free assays (25-fold) compared to Rhod-TMC (143-fold) and RMCIO-2 (150-fold), although these sensitivities did not quite translate in cellular assays. Indeed, when endogenously produced $\mathrm{HOCl}$ was monitored in RAW 264.7 macrophages,

a)

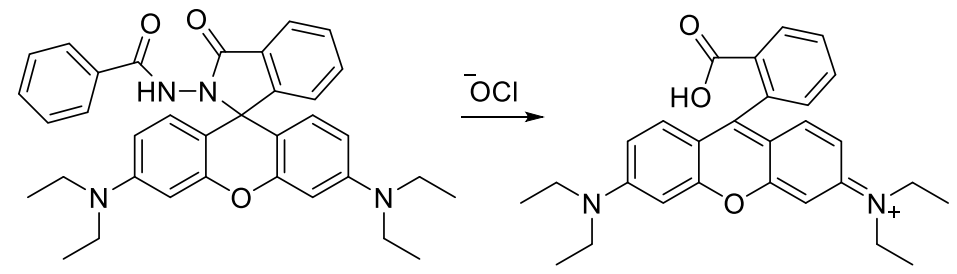

b)

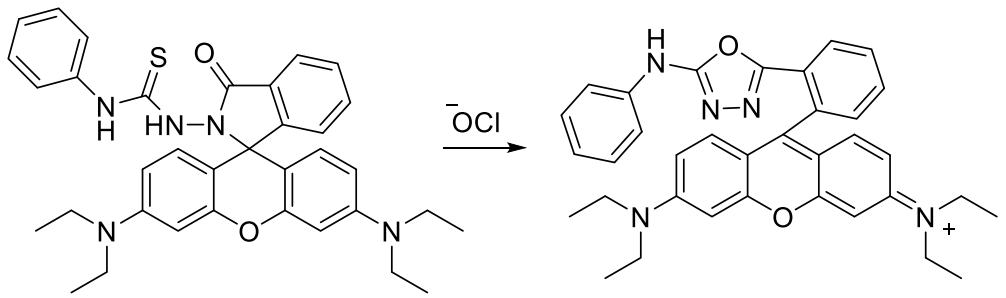

Figure 2-3: Spirolactam ring-opening reaction by ${ }^{-} \mathrm{OCl}$ adapted from ${ }^{[92]}$. 
all three probes showed comparable increases in ratiometric signal. It should, however, be mentioned that the macrophage stimulation was induced by different methods in each case. In the case of Rhod-TMC, the stimulation was achieved with LPS and PMA and the shift in emission signal was imaged qualitatively. For Cou-Rho only LPS was used, while for RMCIO-2 carbonyl cyanide m-chlorophenylhydrazone was chosen. Even though cell-free assays indicated a much higher sensitivity for RMClO-2, the increase in ratiometric signal for Cou-Rho and RMClO-2 was in fact similar. Further studies in HeLa cells with RMCIO-2, equipped with a TPP targeting moiety, revealed preferential accumulation of the sensor in mitochondria. Additionally, the basal levels of $\mathrm{HOCl}$ were studied in several cell lines and were found to be increased in cancer cells (HeLa, A549) compared to normal ones (HEK293, RAW 264.7). Such basic evaluation of cell lines can give valuable insights in how the metabolism of cancer cells may be altered. Yet, comparing different studies may sometimes be difficult, as experimental conditions can differ greatly. Taking into account that the rhodamine ring-opening is used to detect various endogenous analytes, a careful investigation of interferences of other compounds is necessary to estimate the biological significance of the detected signal. Similar cautions should be taken in the case of systems exploiting the oxidation of double bonds, as other strong oxidants (e.g. $\mathrm{ONOO}^{-}$or $\mathrm{HO}^{\circ}$ ) show a comparable reaction behavior. An example for a sensing mechanism based on double-bond oxidation is the coumarin-piperazine (CPBT) platform, which showed a 123-fold increase in ${ }^{-} \mathrm{OCl}$ sensing experiments and a 2.3-fold increase in stimulated RAW 264.7 cells. However, also a small interference with $\mathrm{ONOO}^{-}$was detected in cell-free assays. ${ }^{[105]}$ A more successful example of a sensor with a mitochondria targeting unit that was based on double-bond oxidation (PMN-TPP) showed good selectivity over other ROS, an approximately 80-fold increase in fluorescence

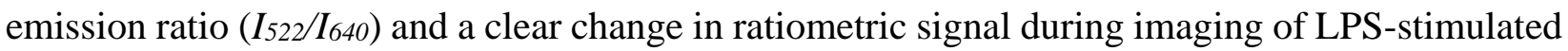
RAW 264.7 macrophages, but was not quantified. In vivo experiments with nude mice receiving a skin-pop injection of LPS followed by PMA were used to monitor the evolution of the two emission signals of PMN-TPP over $60 \mathrm{~min}$. An increase in the ratio ( $\left.\mathrm{I}_{\text {green }} / \mathrm{I}_{\mathrm{red}}\right)$ of fluorescence emission signals was detected, which confirms that ratiometric imaging with PMN-TPP in animal skin is possible (Figure 2-4). ${ }^{[106]}$ Nevertheless, the used excitation wavelength $\left(\lambda_{\mathrm{ex}}=430 \mathrm{~nm}\right)$ is not optimal for animal imaging and is likely to create elevated background fluorescence, especially if other organs than the skin are studied. To improve in vivo imaging, the fluorescence excitation and emission wavelengths of the sensor should be shifted to the NIR region. ${ }^{[51]}$ Another hurdle that may descend from complex biological samples is the interference of hypobromous acid and 
chloramines. These species are generally not tested but exhibit a similar reaction behavior and can therefore interfere with the data obtained.
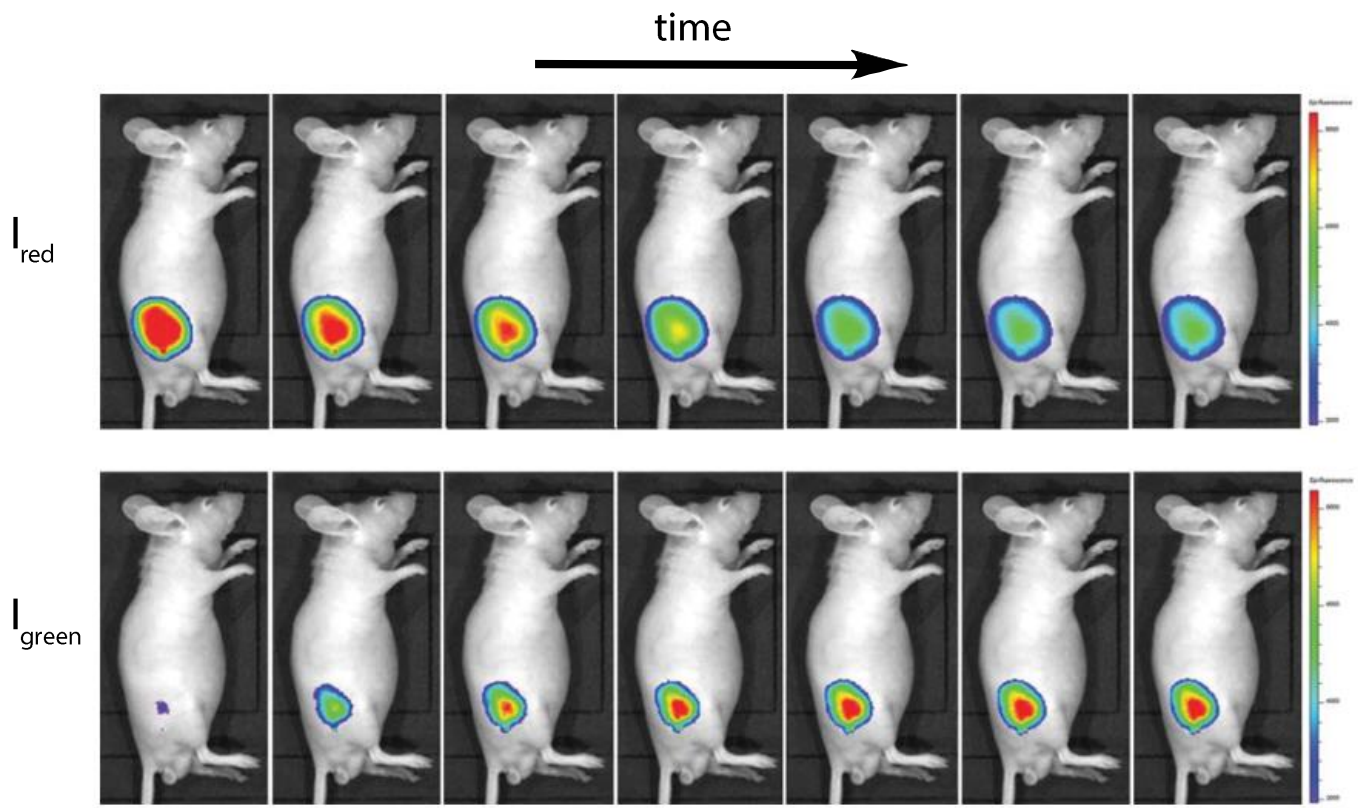

Figure 2-4: Representative fluorescence, pseudo-color images of a nude mouse after skin-pop injection of LPS $\left(100 \mu \mathrm{L} \times 1 \mu \mathrm{g} \mathrm{mL}^{-1}\right)$ for $12 \mathrm{~h}$ and PMA $\left(50 \mu \mathrm{L} \times 1 \mu \mathrm{g} \mathrm{mL}^{-1}\right)$ injection for 30 min. Then a skin-pop injection of PMN-TPP $(50 \mu \mathrm{L} \times 20 \mu \mathrm{M})$ was applied and images were taken using an excitation laser of $430 \mathrm{~nm}$, an emission filter of the DsRed channel ( $\mathrm{I}_{\mathrm{red}}$ ) and an emission filter of the GFP channel (Igreen). Adapted from Xiao et al. with permission. ${ }^{[106]}$ 
Table 2-2: Ratiometric fluorescent probes for the specific detection of $\mathrm{HOCl}$.

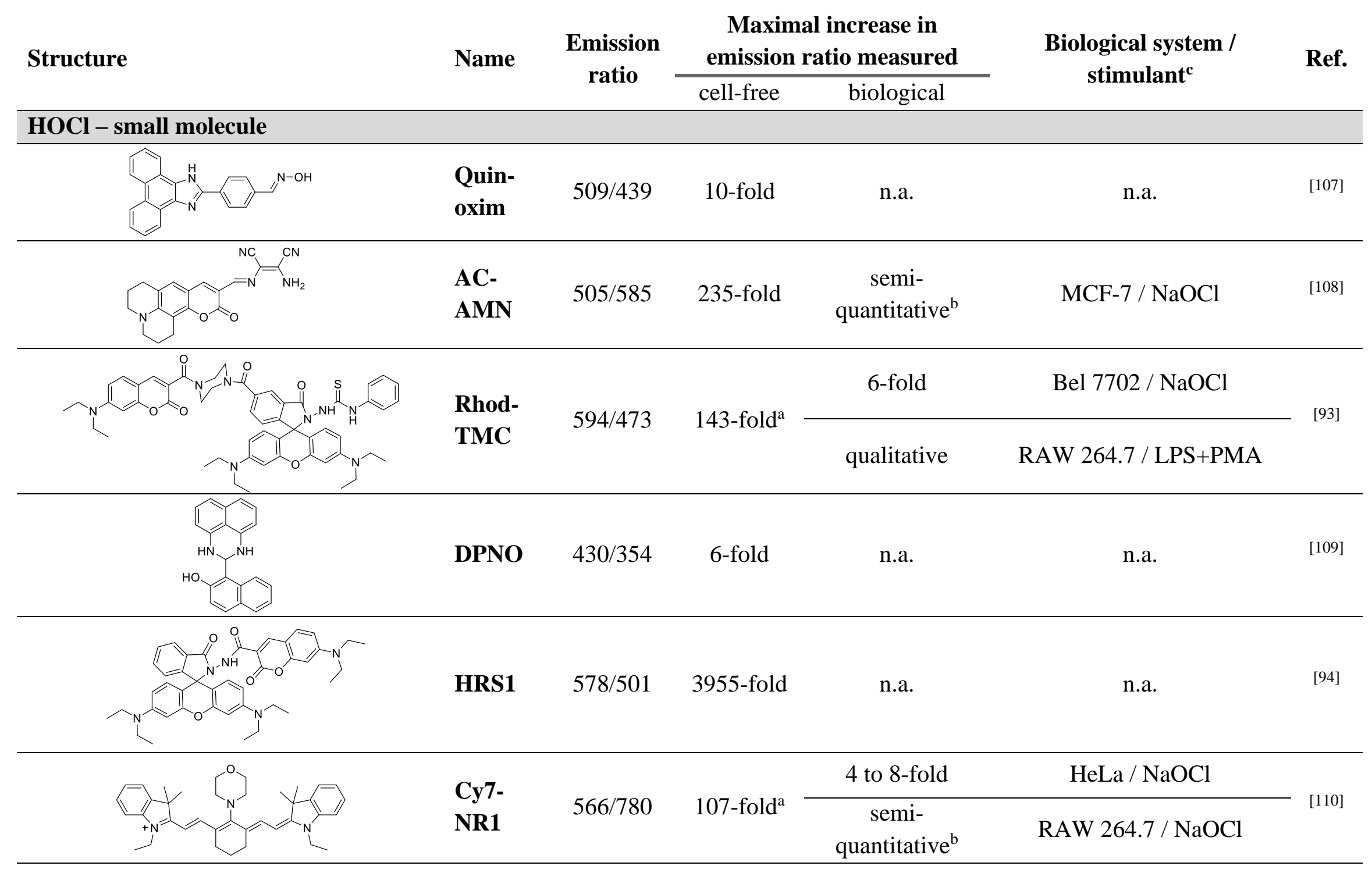




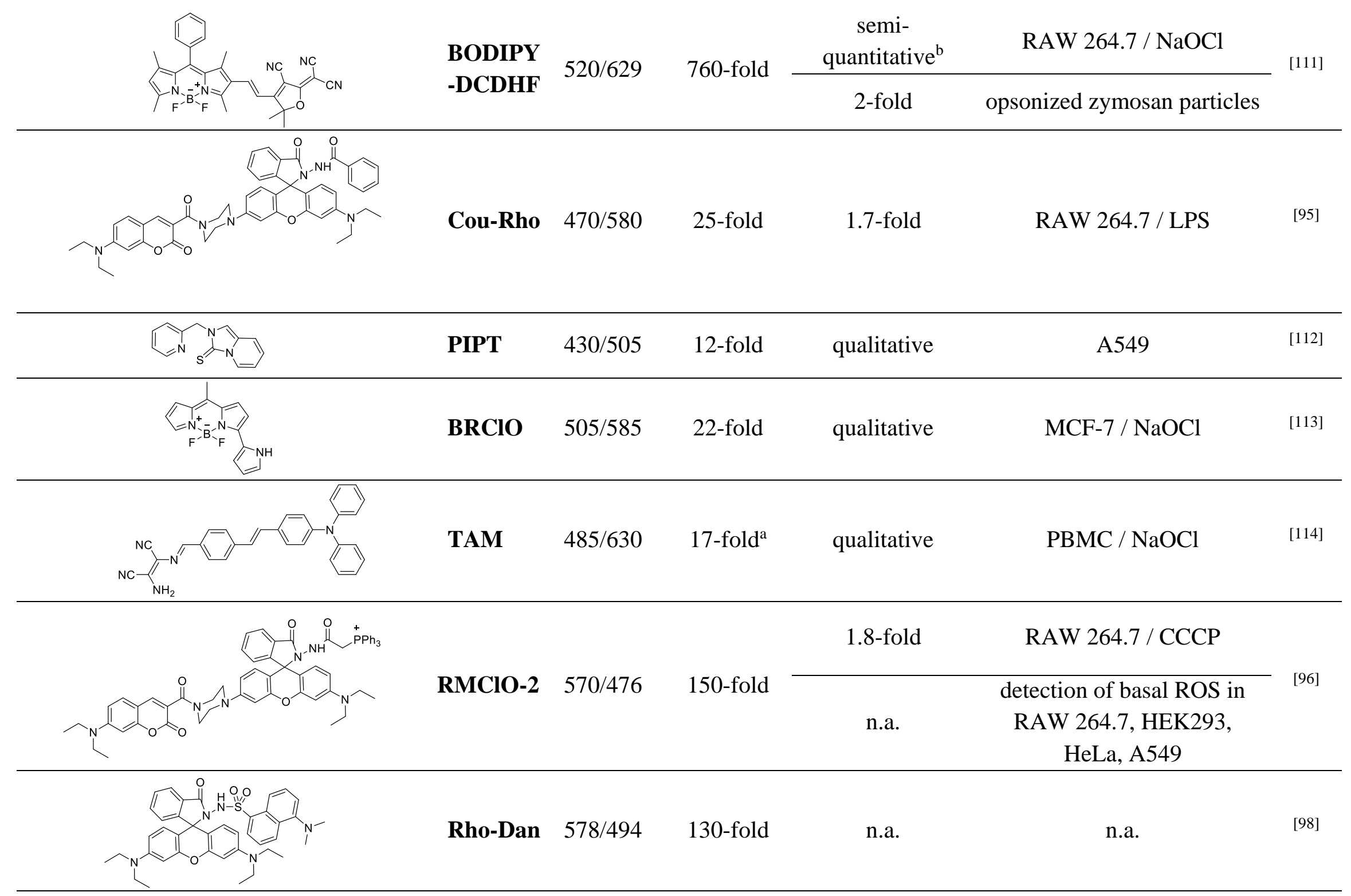




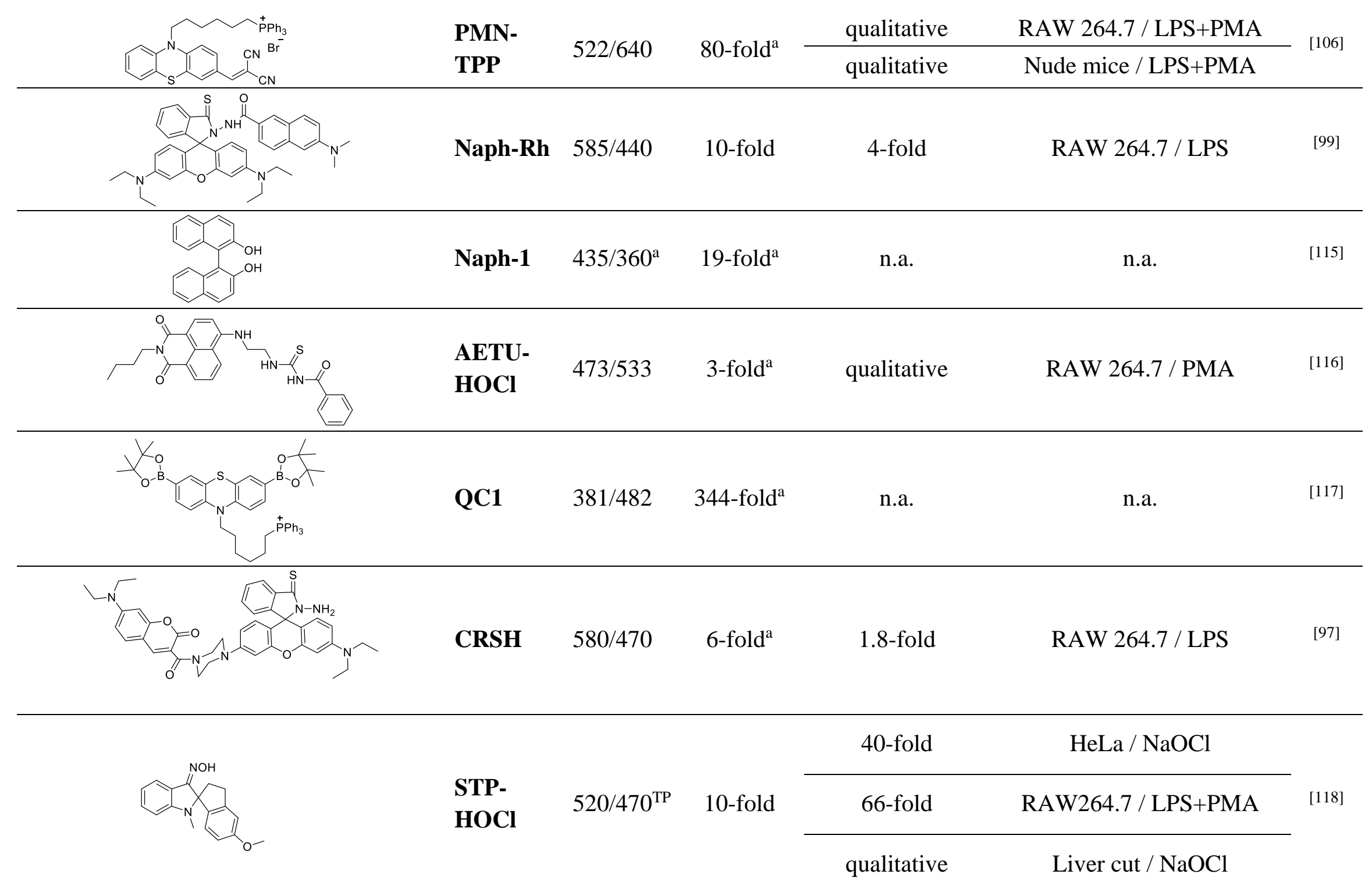




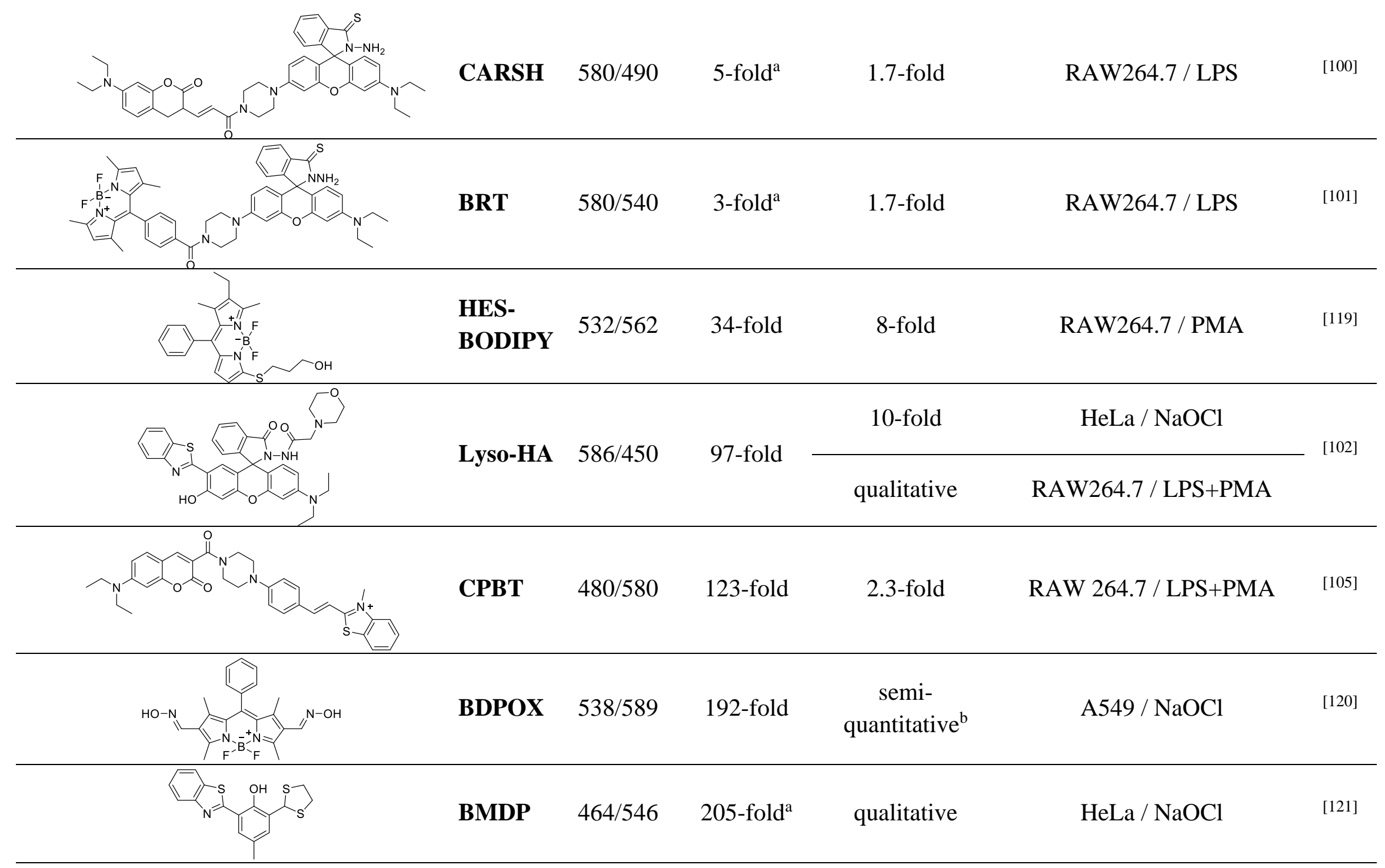




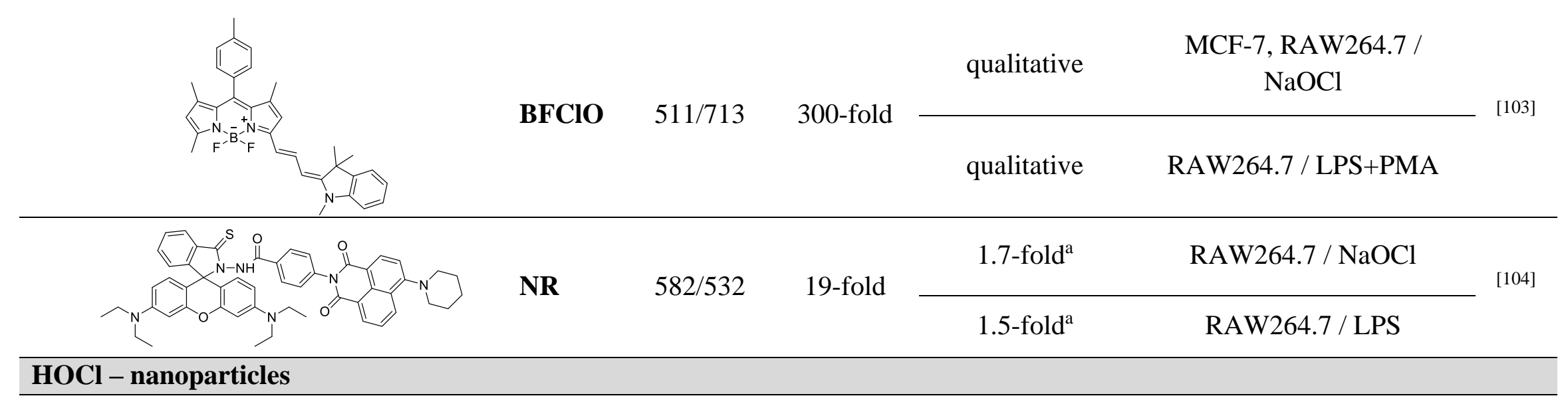

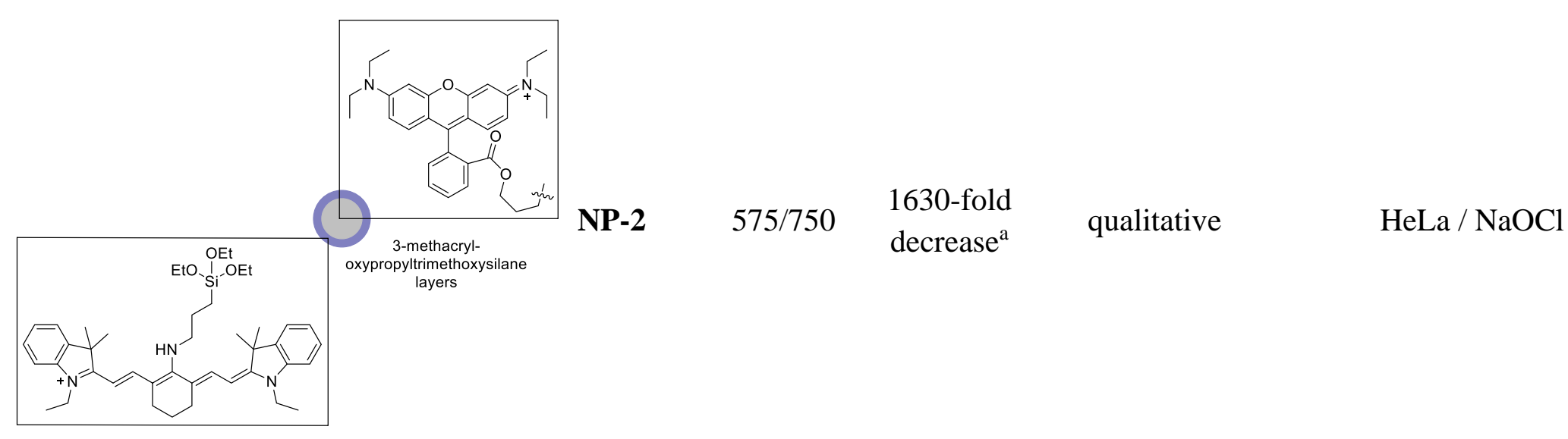

ThioRB-

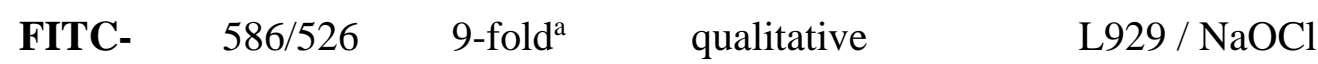

[123] MSN 
${ }^{\mathrm{a}}$ Values calculated from graphs with plot digitizer, ${ }^{\mathrm{b}}$ Color indicating value, but no quantification ${ }^{\mathrm{c}}$ Compound used to induce or mimic stress response, ${ }^{\mathrm{TP}}$ two-photon dye, LPS: lipopolysaccharide, PMA: phorbol-12-myristate-13-acetate, CCCP: carbonyl cyanide $m$ chlorophenylhydrazone, n.a.: not available. 


\subsubsection{Probes for $\mathrm{HO}^{\circ}$}

$\mathrm{HO}^{*}$ is produced by metal-mediated cleavage of $\mathrm{H}_{2} \mathrm{O}_{2}$ and even though only small amounts are generated, this very reactive compound can have severe effects on biological samples, mainly by oxidizing biomolecules. ${ }^{[124]}$ Exploiting the susceptibility of DNA towards oxidative cleavage by $\mathrm{HO}^{\circ}$, a FRET-based ratiometric probe was designed linking two dyes (FAM and TAMRA) through two thymine nucleotides. Despite a promising 40 -fold increase in ratiometric signal of the sensor in cell-free assays, this setup has not found wide-spread use (Table 2-3). ${ }^{[25]}$ The lack of reliable organic turn-on probes to specifically detect $\mathrm{HO}^{*}$ confirms that the high reactivity and short lifetime (in vivo: $\mathrm{t}_{1 / 2}=\sim 10^{-9} \mathrm{~s}$ ) of this radical is problematic. ${ }^{[4]}$ The strong oxidizing power of $\mathrm{HO}^{\bullet}$ also leads to the oxidative cleavage of cyanine dyes, which was investigated by Oushiki et al.. ${ }^{[126]}$ This was the starting point for the design of a coumarin-hemicyanine platform $\mathbf{C o u}-\mathbf{C y}-\mathbf{O H}$ in which a double bond oxidation induces a shift in the emission wavelength but does not lead to degradation. A 210-fold increase of the emission ratio $\left(I_{651} / I_{495}\right)$ was observed and qualitative imaging in PMAstimulated HeLa cells was achieved. ${ }^{[127]}$ Another approach coupled HPF, a traditional fluorophore for $\mathrm{HO}^{\circ}$ and $\mathrm{ONOO}^{-}$, to gold nanocarriers so that the turn-on signal of HPF could be normalized to the constant emission of the nanoparticles. This AuNC-HPF sensor showed selectivity towards $\mathrm{HO}^{\bullet}$ with a small interference from $\mathrm{ONOO}^{-}$, representing a typical example of how turn-on fluorophores can be used in a nanoparticulate ratiometric sensor. ${ }^{[128]}$ 
Table 2-3: Ratiometric fluorescent probes for the specific detection of $\mathrm{HO}^{\circ}$.

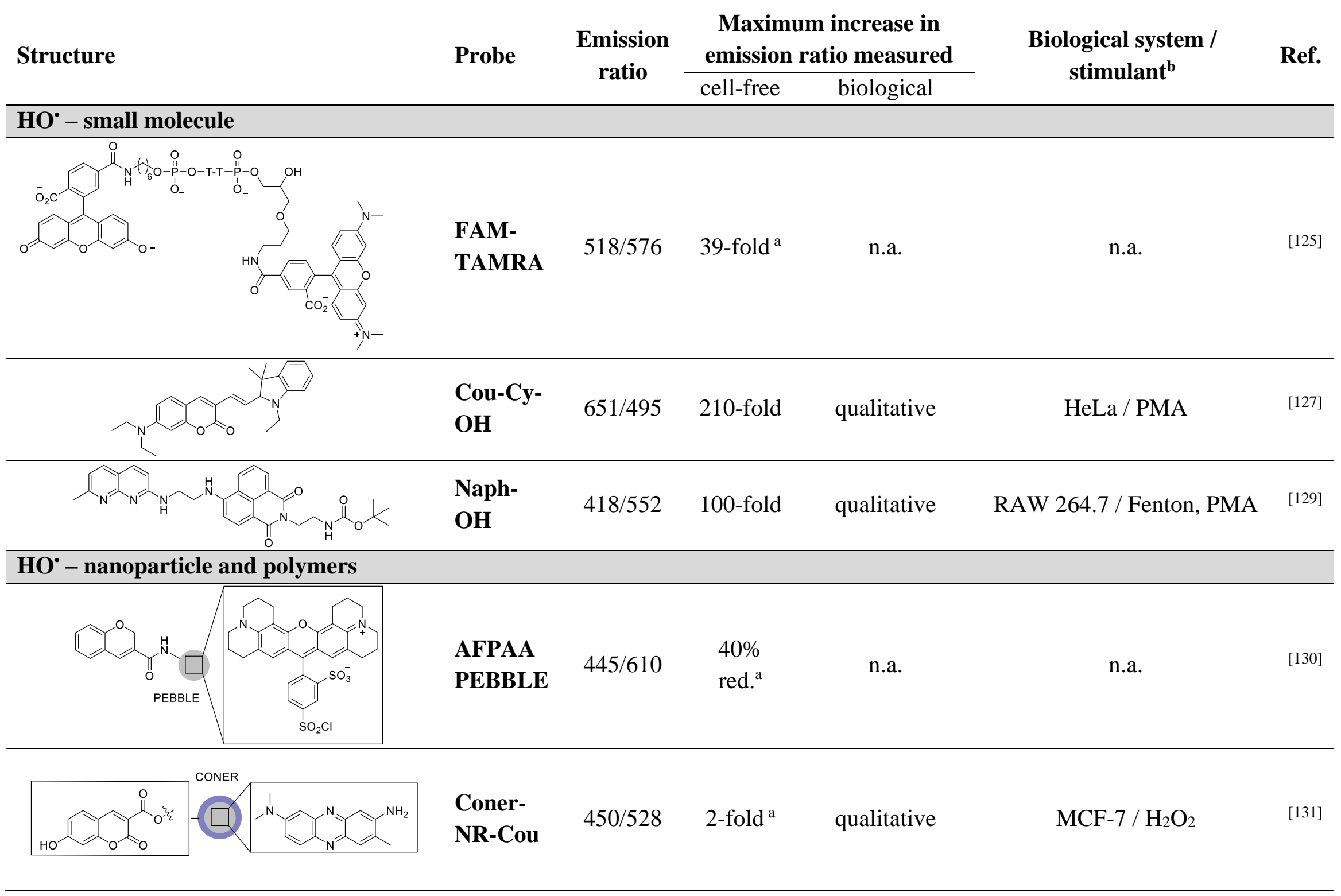




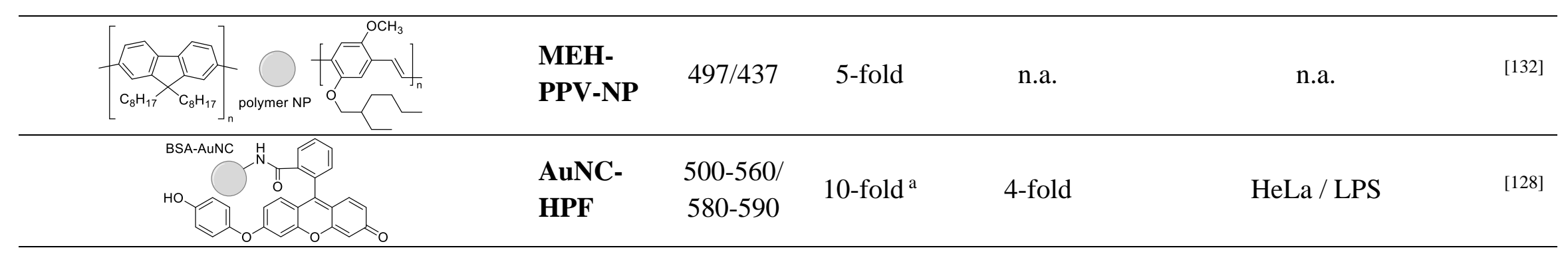

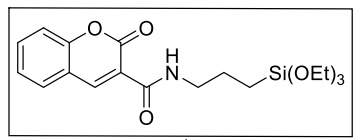

$\mathrm{SiO}_{2} \mathrm{NP}$

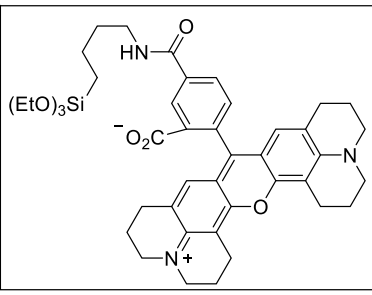

CCAAPTES
$455 / 620$

2-fold

qualitative

HeLa / Fenton

[133]

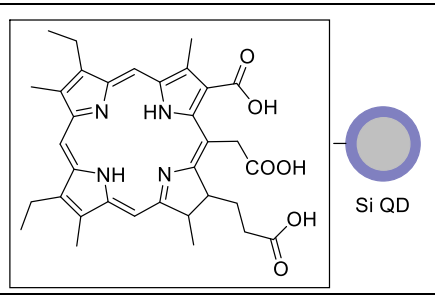

$\begin{array}{lllll}\text { Si Qd } & \text { 660/490 } & \text { 3-fold } & \text { qualitative } & \text { HepG2 - LPS }\end{array}$

${ }^{a}$ Values calculated from graphs with plot digitizer, ${ }^{b}$ Compound used to induce or mimic stress response, LPS: lipopolysaccharide, PMA: phorbol-12-myristate-13-acetate, n.a.: not available. 


\subsubsection{Probes for $\mathrm{O}_{2}{ }^{--}$}

$\mathrm{O}_{2}{ }^{*}$ imaging also suffers from similar problems; namely, specific fluorophores are difficult to design and the lifetime of the molecule is short $\left(\mathrm{t}_{1 / 2}=10^{-3} \mathrm{~s}\right) .{ }^{[135]}$ Interestingly, it is not the primary ROS itself that is the most dangerous reactive species but the fast dismutation of $\mathrm{O}_{2}{ }^{\circ}{ }^{-}$to $\mathrm{H}_{2} \mathrm{O}_{2}$ by $\mathrm{SOD}$, and it is its diffusion-controlled recombination with $\mathrm{NO}^{\circ}$ to $\mathrm{ONOO}^{-}$that render it difficult to study. The widely used and commercially available fluorophore HE, for example, is known to not only react with $\mathrm{O}_{2}{ }^{*}$ leading to $2-\mathrm{OH}-\mathrm{E}^{+}$, but to also form a second oxidation product $\left(\mathrm{E}^{+}\right)$with other ROS that has similar spectral properties. Due to the overlapping fluorescence emissions, these two products can only be distinguished by HPLC analysis. ${ }^{[136,137]}$ Nevertheless, HE has been used in various ratiometric sensors (Table 2-4). Tian and collaborators have designed a nanosensor in which HE was coupled to the surface of a carbon dot (CD-HE), allowing the turn-on response to be normalized to the constant emission of the nanoparticle (I570-630/I500-560). A 3-fold change in ratiometric signal was observed in cell-free assays, and LPS-treated HeLa cells led to a doubling of the ratio. ${ }^{[138]}$ The same group also introduced a nanoprobe to simultaneously detect $\mathrm{pH}$ and $\mathrm{O}_{2}{ }^{-}$ in the mitochondria. For this purpose, the $\mathrm{pH}$ and $\mathrm{O}_{2}{ }^{\circ-}$ sensors (FITC, $\lambda_{\mathrm{em}, \max }=520 \mathrm{~nm}$ and HE, $\lambda_{\mathrm{em}, \max }=630 \mathrm{~nm}$ ) and a mitochondria targeting moiety were grafted onto the surface of quantum dots $\left(\lambda_{\text {em,max }}=800 \mathrm{~nm}\right)$. An improved increase in ratiometric signal (5-fold) compared to CD-HE was observed. Addition of $\mathrm{O}_{2}{ }^{-*}$ to RAW 264.7 macrophages triggered a 3.7-fold increase in fluorescence emission ratio and revealed a concomitant increase in $\mathrm{pH}^{\left[{ }^{[139]}\right.}$ Our group has recently proposed a sensor to detect extracellular $\mathrm{O}_{2}{ }^{*-}$ with improved flexibility. In this scaffold the reference dye Chromis500 was combined with the turn-on sensor ${ }^{[40]}$ hydrocyanine5. High flexibility was achieved by using a double stranded DNA analogue that allowed to combine the two fluorophores in a modular fashion. An up to 2-fold increase in the ratiometric signal was observed in cell-free assays, as well as with Caco-2 cells stimulated with $\mathrm{H}_{2} \mathrm{O}_{2} \cdot{ }^{[140]}$ 
Table 2-4: Ratiometric fluorescent probes for the specific detection of $\mathrm{O}_{2}{ }^{\circ-}$.

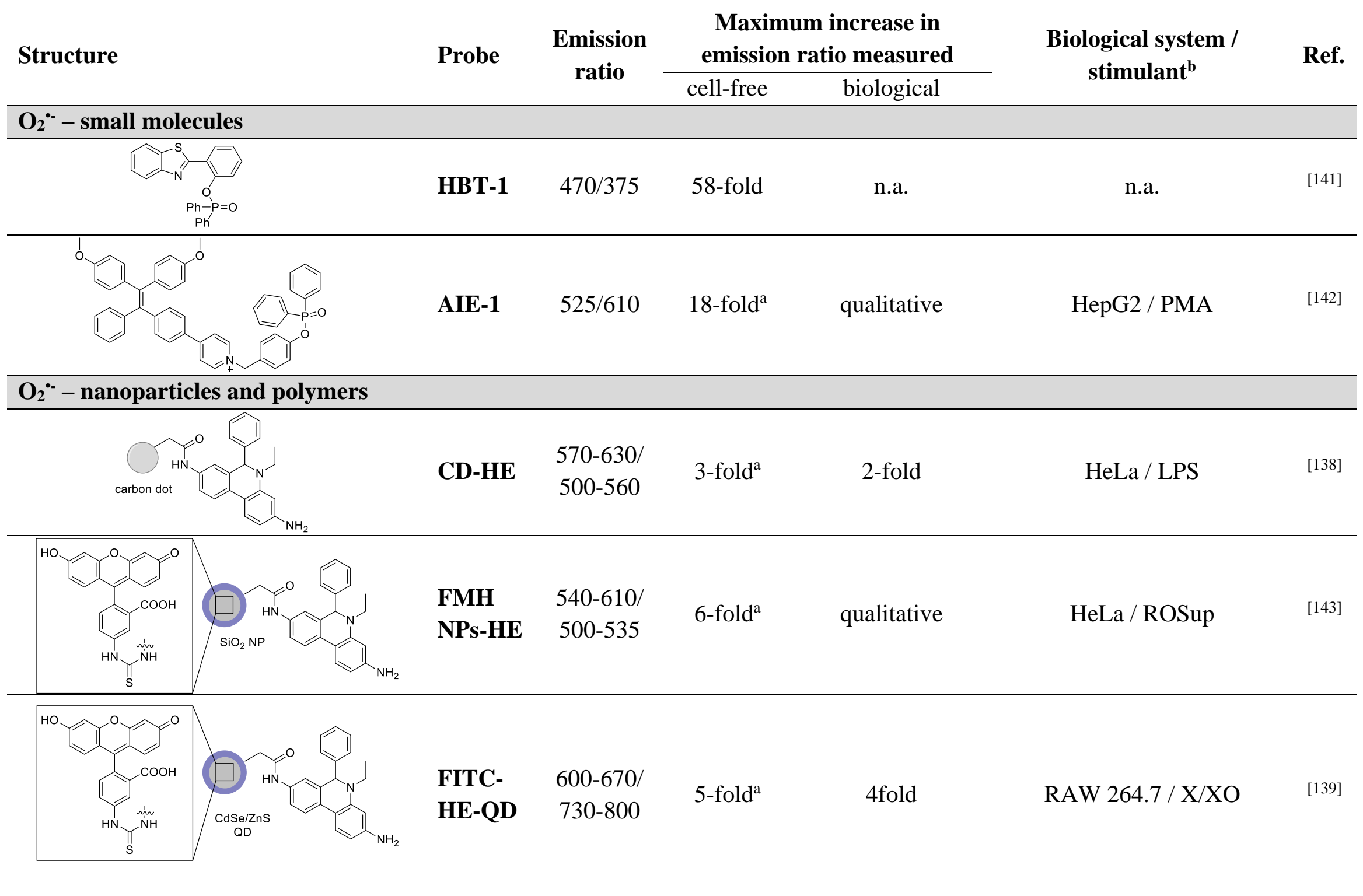




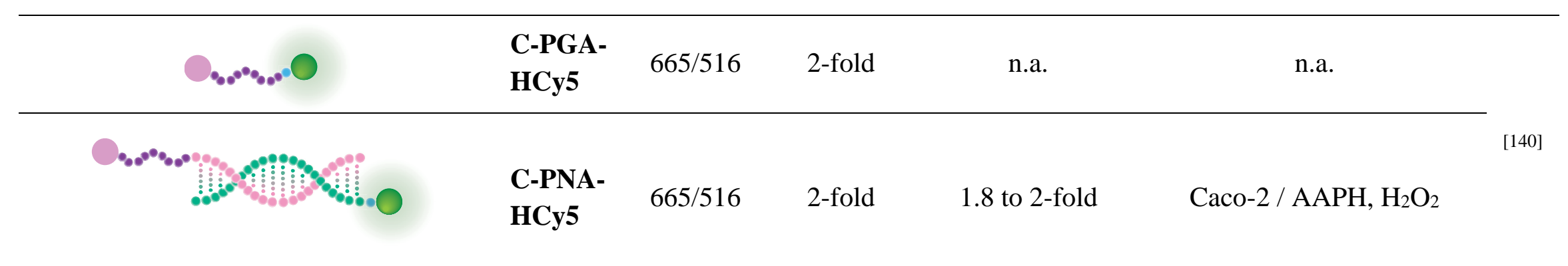

${ }^{\mathrm{a}}$ Values calculated from graphs with plot digitizer, ${ }^{\mathrm{b}}$ Compound used to induce or mimic stress response, LPS: lipopolysaccharide, AAPH: 2,2'-Azobis(2-amidinopropane) dihydrochloride, n.a.: not available. 


\subsubsection{Probes for NO}

$\mathrm{NO}^{\bullet}$ has been recognized as an important messenger in the body for a long time. The physiological and pathological functions of this gaseous free radical have broadened extensively and now include cardiovascular signaling, neurological messenger functions and immune responsive activities. Two main classes of fluorescent sensors have been introduced, metal-based or FRET-based, both of them relying on the specific reaction of $\mathrm{NO}^{\bullet}$ to modulate fluorescence emission. ${ }^{[42,144]}$ In metalbased probes, the direct reaction with the metal center or close to the metal site is responsible for the change in fluorescence. ${ }^{[145]}$ To the best of our knowledge, however, this approach has not found wide-spread translation into ratiometric probe set-ups for NO. The other class of sensors includes fluorophores equipped with a functional reactive group towards NO` and are mostly FRET-based (Table 2-5). The spirolactam ring opening, which is also used in ion and $\mathrm{HOCl}$ sensing, has been adapted to be specific towards $\mathrm{NO}^{\cdot}{ }^{[92]}$ Instead of a hydrazine, an $o$-phenylamine was introduced, leading to the formation of benzotriazole upon reaction with $\mathrm{NO}^{\bullet}$ and followed by hydrolysis (Figure 2-5). Two FRET-based approaches have been introduced where ring-opening leads to a decrease of the donor and an increase of the rhodamine emission due to FRET. For instance, in Cou-Rho-NO coumarin was used as donor dye, whereas BODIPY was chosen in BRP-NO. The sensors showed a strong increase (>250-fold) in fluorescent emission ratio and ratiometric imaging in cells was achieved in both cases qualitatively. Further quantitative cell experiments would be necessary to more accurately estimate the potential of these probes for biological applications. ${ }^{[146,147]}$ Solubility issues of purely organic probes can often hamper the translation of novel imaging probes into in vitro or in vivo systems. With nanoparticles-based probes, the use of organic solvents can be avoided as particles suspension in aqueous media can be used. An example

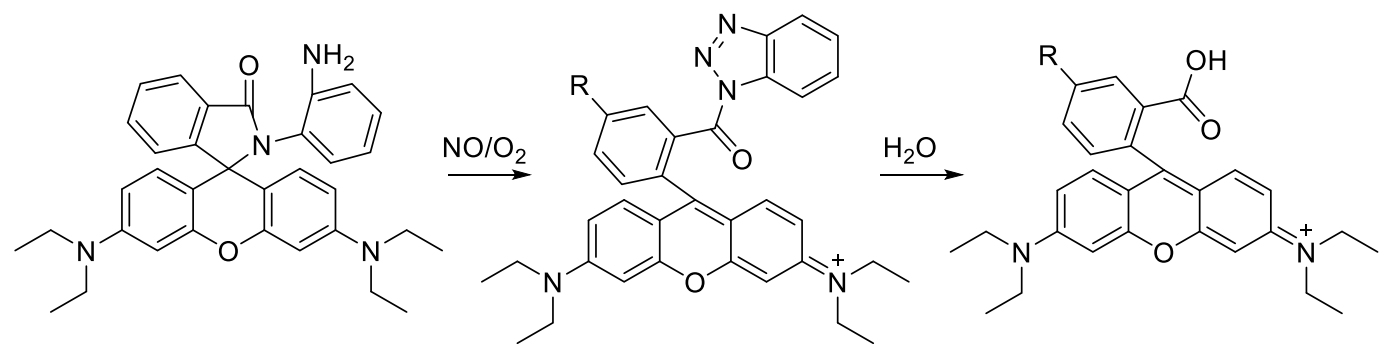

Figure 2-5: Spirolactam ring opening reaction specific for NO adapted from ${ }^{[146]}$.

of this is CD-naph, a carbon dot-based system to which an organic naphthalimide with an $o$ phenylendiamine group was anchored. In this setup, the reaction with NO lead to the formation of an aryltriazole that enabled FRET from the carbon dot to the naphthalimide moiety. ${ }^{[148]}$ 


\subsubsection{Probes for $\mathrm{ONOO}^{-}$}

$\mathrm{ONOO}^{-}$is the product of the diffusion-controlled reaction between $\mathrm{NO}^{-}$and $\mathrm{O}_{2}{ }^{-}$, and plays an important role in signal transduction and antimicrobial activities. ${ }^{[16]}$ Nonetheless, it can also have deleterious effects at elevated concentrations, such as the oxidation of proteins, DNA or lipids. ${ }^{[149]}$ The fast reaction with arylboronates offers an excellent opportunity for preparing probes to study ONOO$^{-}$in biological systems. ${ }^{[75,150]}$ Surprisingly, only two $\mathrm{ONOO}^{-}$-detecting ratiometric probes have been published so far using arylboronates, ${ }^{[151,152]}$ and only a qualitative turn-on response has been reported in cell-based systems. The strong oxidizing power of $\mathrm{ONOO}^{-}$has been harnessed to create many other set-ups that are based on a selective oxidation of the sensor, i.e. the oxidation of activated double bonds (Table 2-6). Exploiting the aforementioned pattern, HMBT-Py and $\mathbf{F}_{482}$ have shown $>50$-fold increases in cell-free experiments and good selectivity over other ROS and reactive sulfur species (RSS). However, the potential of these sensors differed greatly from each other when tested in more complex biological systems. While for HMBT-Py the potential could not be confirmed in HeLa nor in A549 cells, ${ }^{[153]} \mathbf{F}_{482}$ was successfully translated to cellular systems, enabling the detection of endogenous $\mathrm{ONOO}^{-}$in LPS+PMA-stimulated RAW 264.7 macrophages and THP-1 cells. Furthermore, imaging of inflammation in the mouse peritoneal cavity and in mouse paws was achieved with $\mathbf{F}_{\mathbf{4 8 2}}$, resulting in 10-fold and 5-fold increases, respectively. ${ }^{[154]}$ These two cases exemplify once again the discrepancies that can be found in performance measurements done in simplified sensing setups compared to more complex and biologically relevant environments, such as cell cultures or in vivo settings. Nevertheless, to improve in vivo imaging, sensors with emission wavelength in the NIR will be needed in order to minimize tissue damage and cell autofluorescence. ${ }^{[51]}$ 
Table 2-5: Ratiometric fluorescent probes for the specific detection of NO.

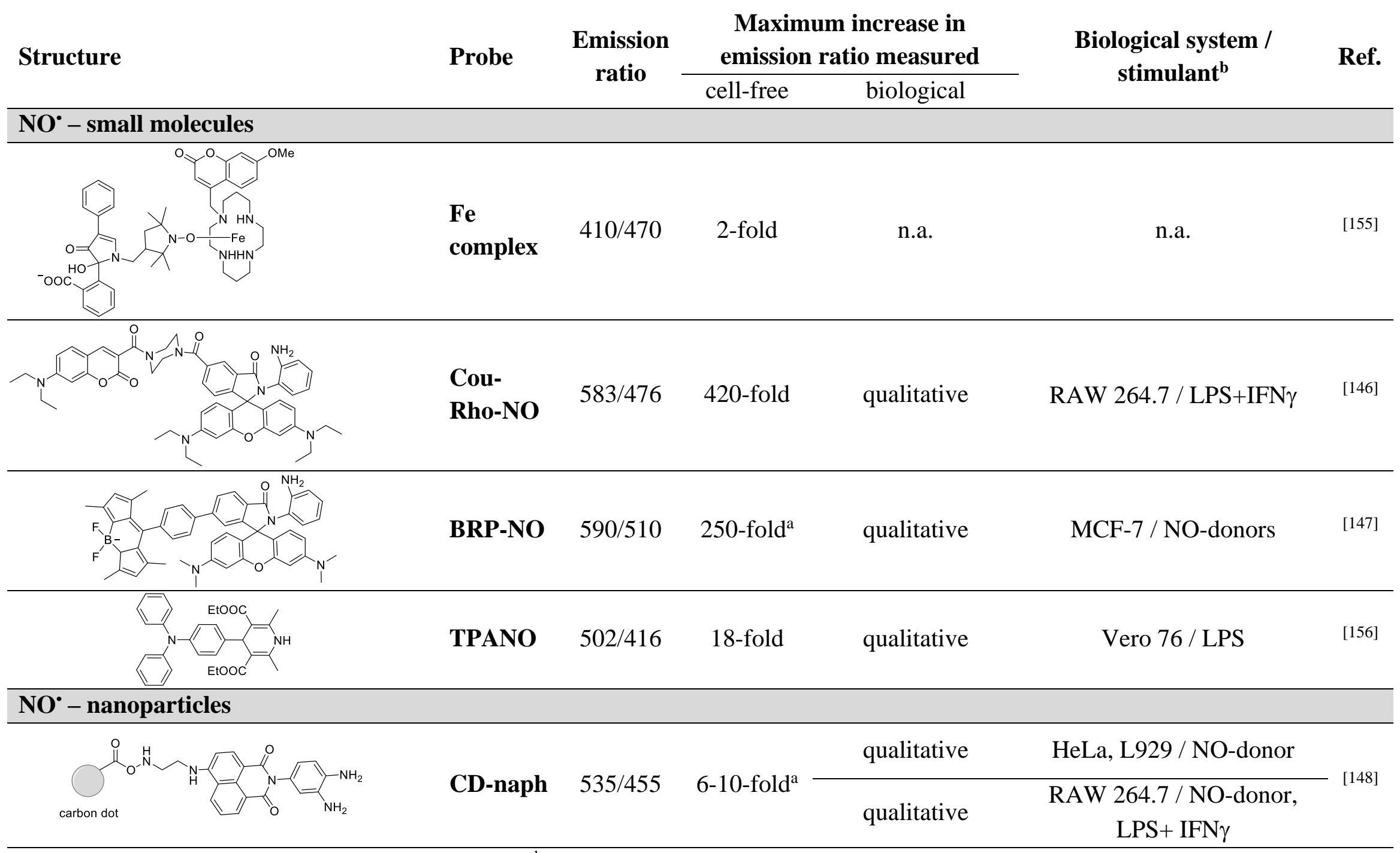

${ }^{\mathrm{a}}$ Values calculated from graphs with plot digitizer, ${ }^{\mathrm{b}}$ Compound used to induce or mimic stress response, LPS: lipopolysaccharide, IFN $\gamma$ : interferon $\gamma$, n.a.: not available. 
A recent example of a two-photon ratiometric turn-on probe exemplifies the power of fluorescent imaging agents emitting in the near infrared. After screening 19 different fluorophores, the most sensitive and resistant candidates were combined as FRET pairs in MITO-CC. Evaluation of the probe in cell-free assays showed a 93-fold enhancement of ratiometric signal (I473/I651), a fast response (within $20 \mathrm{~s}$ ) with a low detection limit $(11.30 \mathrm{nM})$ and good selectivity over other ROS and RSS. MITO-CC successfully detected ONOO- in HepG2 and RAW 264.7 cells as observed with a two-photon confocal microscope, and ex vivo stimulation of rat liver cuts with LPS resulted in a clear increase of ratiometric signal. Finally, leg skin sections of Kumming mice revealed a 3.1fold increase in signal after LPS-induced inflammation (Figure 2-6). ${ }^{[157]}$ Overall, the recently developed probes for $\mathrm{ONOO}^{-}$detection appear very promising, and further applications in biological systems are expected to arise and confirm their potential.

A
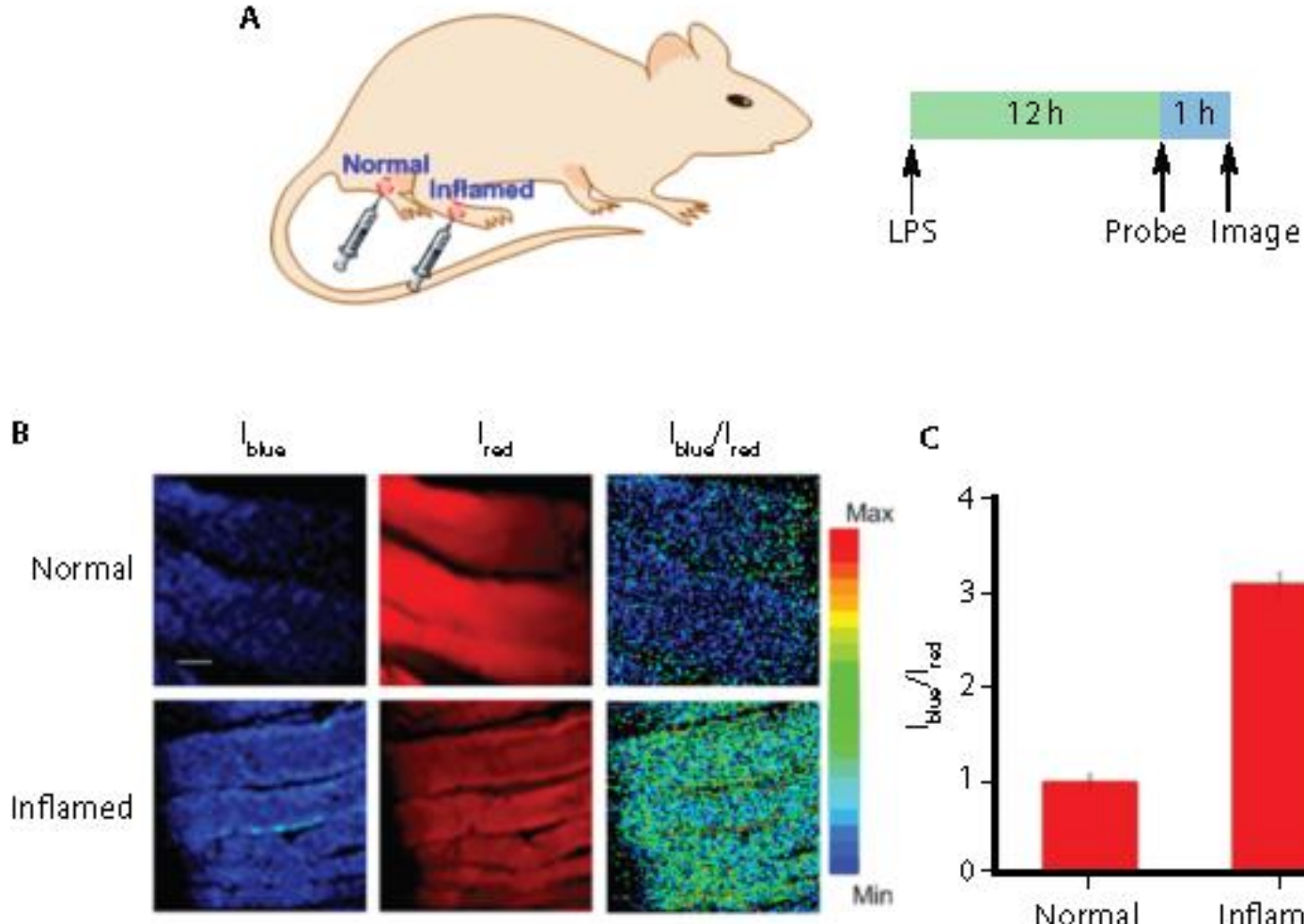

C

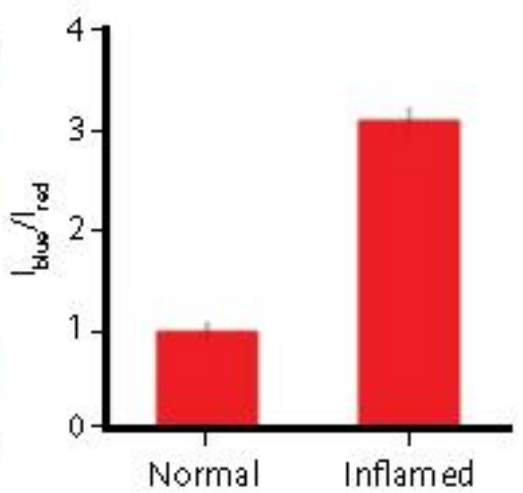

Figure 2-6: Imaging of $\mathrm{ONOO}^{-}$with MITO-CC using two-photon confocal microscopic fluorescence images. (A) Subcutaneous injection of LPS $(200 \mu \mathrm{L} \times 1 \mu \mathrm{g} / \mathrm{mL})$ into right leg of mice. After $12 \mathrm{~h}$, MITO-CC $(20 \mu \mathrm{L} \times 500 \mu \mathrm{M})$ was subcutaneously injected. After $1 \mathrm{~h}$, the leg skin of mice was sectioned after being anaesthetized. (B) Fluorescence images of MITO-CC in the normal and inflamed tissues. (C) Average $F_{\text {blue }} / F_{\text {red }}$ intensity ratios in panel B. Blue channel, $\lambda_{\mathrm{em}}=460-500 \mathrm{~nm}$; red channel, $\lambda_{\mathrm{em}}=605-680 \mathrm{~nm}$. $\lambda_{\mathrm{ex}}=800 \mathrm{~nm}$. Scale bar: $200 \mu \mathrm{m}$. Redrawn from Cheng et al. with permission. ${ }^{[157]}$ 
Table 2-6: Ratiometric fluorescent probes for the specific detection of $\mathrm{ONOO}^{-}$.

\begin{tabular}{|c|c|c|c|c|c|c|}
\hline \multirow[t]{2}{*}{ Structure } & \multirow[t]{2}{*}{ Probe } & \multirow{2}{*}{$\begin{array}{c}\text { Emissio } \\
\text { n ratio }\end{array}$} & \multicolumn{2}{|c|}{$\begin{array}{l}\text { Maximum increase in } \\
\text { emission ratio measured }\end{array}$} & \multirow{2}{*}{$\begin{array}{c}\text { Biological system / } \\
\text { stimulant }^{\mathrm{b}}\end{array}$} & \multirow[t]{2}{*}{ Ref. } \\
\hline & & & cell-free & biological & & \\
\hline & BOD-Se & $680 / 572$ & 225 -fold & n.a. & n.a. & [158] \\
\hline & P2 & $480 / 580$ & 25-fold ${ }^{\mathrm{a}}$ & n.a. & n.a. & [159] \\
\hline & & & & qualitative & WI38 VA13 / ONOO- & \\
\hline & CHCN & $515 / 635$ & $350-$ fold $^{\mathrm{a}}$ & qualitative & $\begin{array}{c}\text { RAW } 264.7 / \\
\text { LPS+IFN } \gamma+\text { PMA }\end{array}$ & [160] \\
\hline & $\begin{array}{l}\text { HMBT- } \\
\text { Py }\end{array}$ & $545 / 645$ & 30-fold ${ }^{\mathrm{a}}$ & no increase & HeLa, A549 / SIN-1 & [153] \\
\hline
\end{tabular}




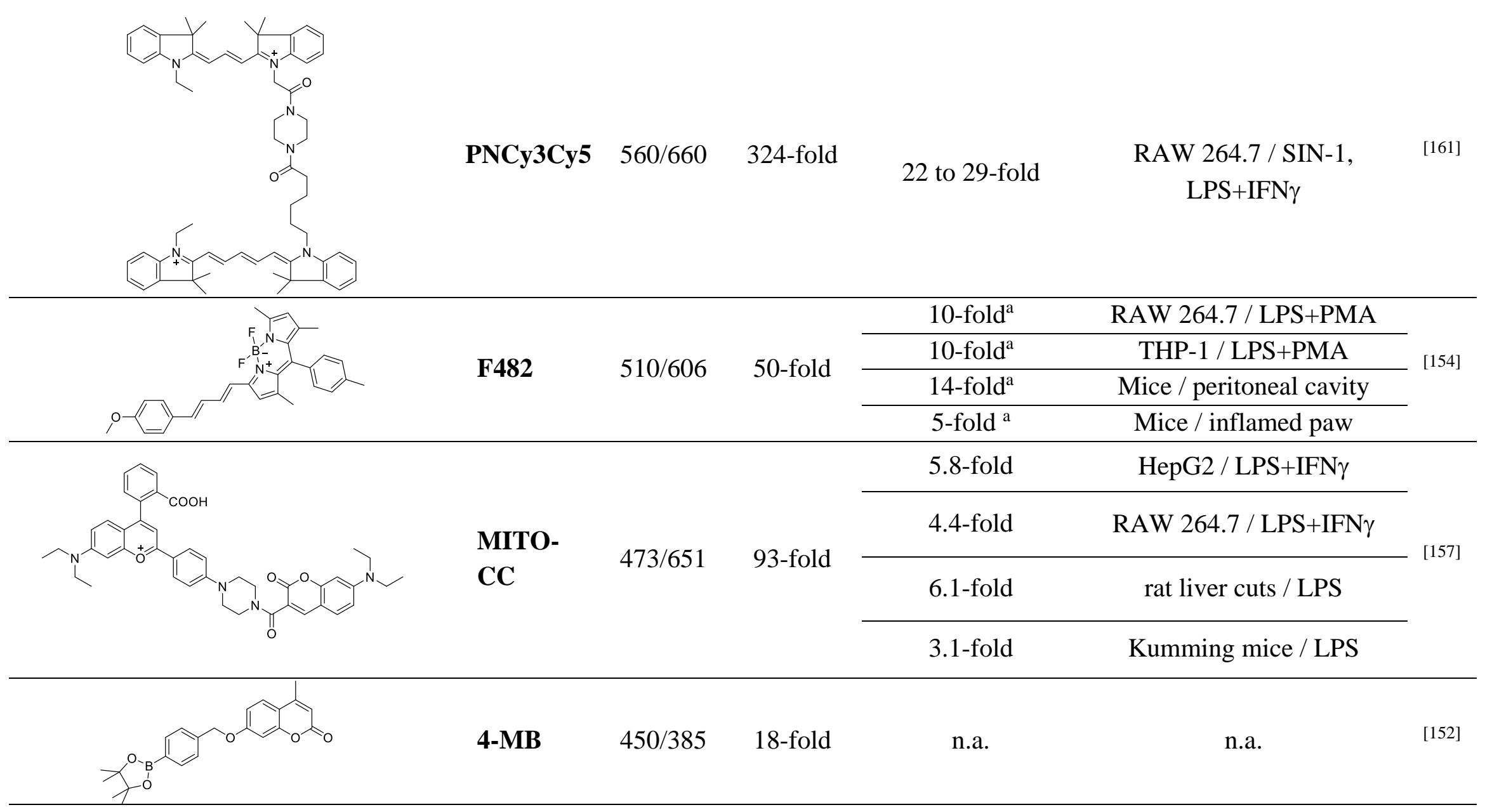




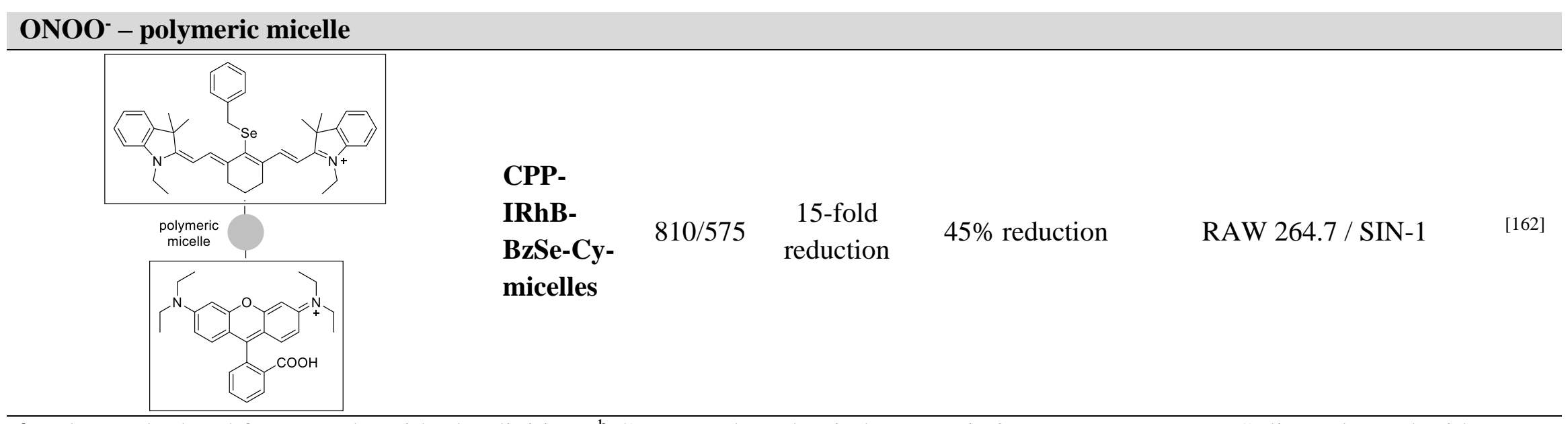

${ }^{\mathrm{a}}$ Values calculated from graphs with plot digitizer, ${ }^{\mathrm{b}}$ Compound used to induce or mimic stress response, LPS: lipopolysaccharide, IFN $\gamma$ : interferon $\gamma$, PMA: phorbol-12-myristate-13-acetate, SIN-1: 3-morpholino-sydnonimine 


\subsubsection{Probes for HNO}

$\mathrm{HNO}$ is the one-electron reduced and protonated form of $\mathrm{NO}^{\circ}$. It was not until evidence emerged that HNO may be produced endogenously, that its role in biological systems began to be investigated more thoroughly. ${ }^{[163]}$ As for the other ROS, HNO was discovered to participate in a wide range of processes, such as cardiovascular regulation, immune system modulation and neuronal activity. ${ }^{[17]}$ Similar to the probes for NO', HNO-sensing strategies have focused on either metal-based approaches or modified fluorophores, with only few ratiometric probes published up

to date (Table 2-7). ${ }^{[164-167]}$ Among these, two-photon dye was combined with a FRET system to create the sensor P-Np-Rhod. Ratiometric imaging in HeLa cells and in frozen rat liver cuts showed a clear shift in emission wavelength, but was not quantified. ${ }^{[167]}$

\subsection{Ratiometric fluorescent probes to detect $\mathrm{hROS}$ and multiple ROS}

Not all ratiometric sensors aim at detecting a single reactive species; some of them may also target a whole group of molecules, such as highly reactive oxygen species (ONOO-, $\mathrm{HOCl}$ and $\mathrm{HO}^{*}$, Table 2-8). Shuhendler et al. introduced a semiconducting-based nanosensor that was equipped with a fluorescent NIR sensor for $\mathrm{ONOO}^{-}$and $\mathrm{HOCl}$, a chemiluminescent $\mathrm{H}_{2} \mathrm{O}_{2}$ substrate and the hepatocyte targeting moiety galactose. An 11-fold increase in ratiometric signal ( $\left.\mathrm{I}_{680} / \mathrm{I}_{820}\right)$ was detected with this chemiluminescent sensor, and selective imaging of $\mathrm{H}_{2} \mathrm{O}_{2}$ was achieved. This nanosensor was successfully applied to a study of drug-induced hepatotoxicity in mice (using the analgesic and anti-pyretic acetaminophen, and the anti-tuberculosis agent isoniazid), where a 1.8and 1.5-fold increase in ratiometric signal was detected, respectively. ${ }^{[168]}$ This study shows that NIR dyes are valuable tools to better understand the roles of ROS in animal models in a noninvasive manner. 
Table 2-7: Ratiometric fluorescent probes for the specific detection of HNO.

\begin{tabular}{|c|c|c|c|c|c|c|}
\hline \multirow{2}{*}{ Structure } & \multirow{2}{*}{ Probe } & \multirow{2}{*}{$\begin{array}{l}\text { Emission } \\
\text { ratio }\end{array}$} & \multicolumn{2}{|c|}{ Increase in emission ratio } & \multirow{2}{*}{$\begin{array}{c}\text { Biological system / } \\
\text { stimulant }^{c}\end{array}$} & \multirow{2}{*}{ Ref. } \\
\hline & & & cell-free & biological & & \\
\hline \multicolumn{7}{|c|}{ HNO - small molecules } \\
\hline & $\begin{array}{l}\text { Naph- } \\
\text { HNO }\end{array}$ & $546 / 418$ & 4-fold ${ }^{\mathrm{a}}$ & $\begin{array}{c}\text { semi- } \\
\text { quantitative }^{b}\end{array}$ & RAW 264.7 / AS & [164] \\
\hline & $\mathbf{C F}$ & $517 / 470$ & $5-$ fold $^{\mathrm{a}}$ & qualitative & HeLa / AS & {$[165]$} \\
\hline & HBT & $460 / 380$ & 10-fold & n.a. & n.a. & [166] \\
\hline & & & & qualitative & HeLa / AS & \\
\hline & Rhod & $P$ & & qualitative & rat liver cuts / AS & \\
\hline & $\begin{array}{l}\text { Bod- } \\
\text { Cou- } \\
\text { HNO }\end{array}$ & $463 / 592$ & 63-fold & qualitative & SMMC-7721 / AS & [169] \\
\hline
\end{tabular}

${ }^{\mathrm{a}}$ Values calculated from graphs with plot digitizer, ${ }^{\mathrm{b}}$ Color indicating value, but no quantification, ${ }^{\mathrm{c}}$ compound used to induce or mimic stress response ${ }^{\mathrm{TP}}$ two-photon probe, AS Angeli's salt 
Table 2-8: Ratiometric fluorescent probes for the specific detection of hROS and multi ROS.

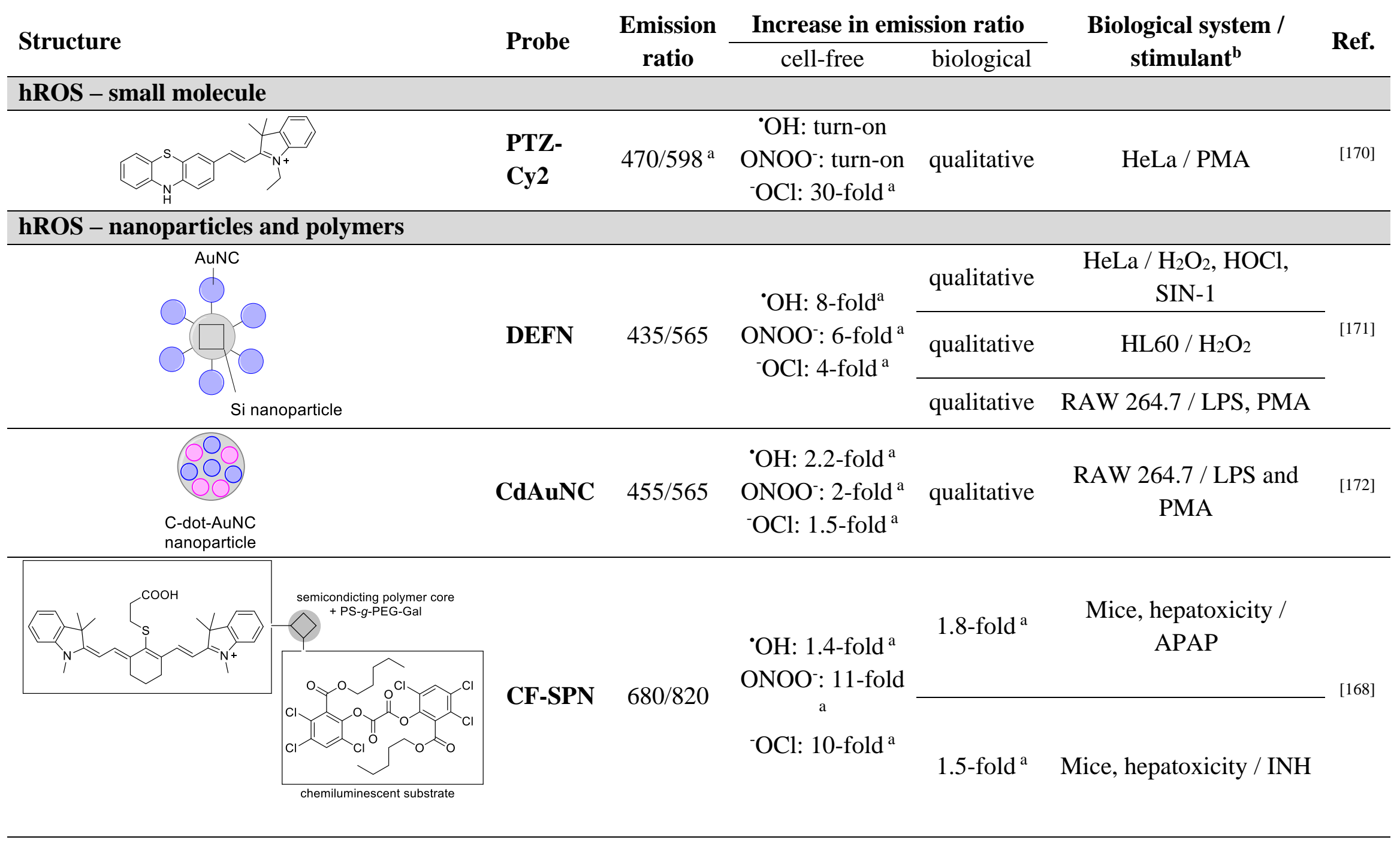




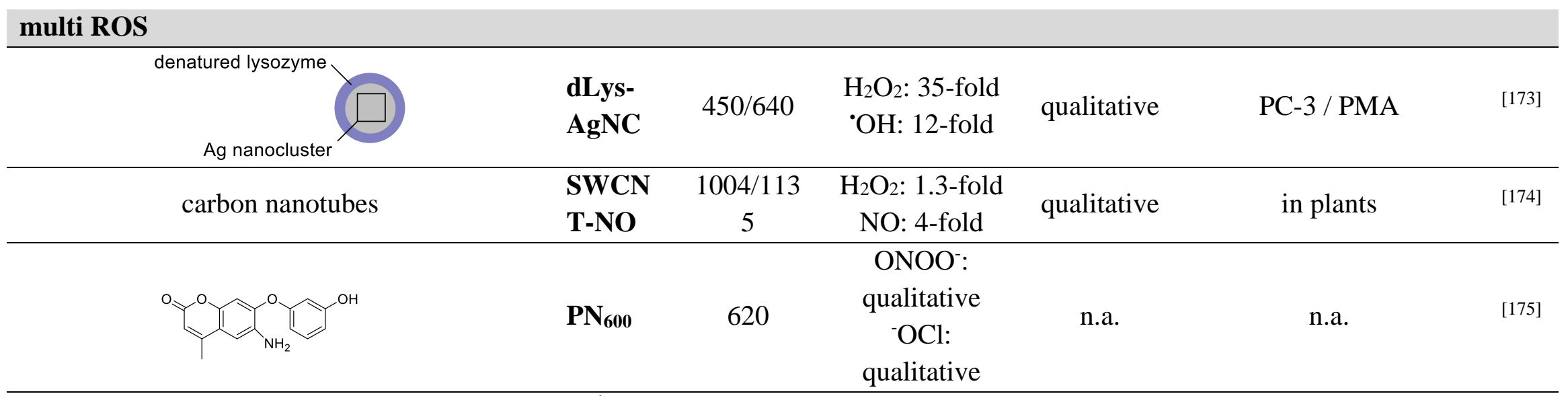

${ }^{\mathrm{a}}$ Values calculated from graphs with plot digitizer, ${ }^{\mathrm{b}}$ Compound used to induce or mimic stress response, LPS: lipopolysaccharide, PMA: phorbol-12-myristate-13-acetate, SIN-1: 3-morpholino-sydnonimine, APAP: analgesic and anti-pyretic acetaminophen, INH: isoniazid. 


\subsection{Ratiometric fluorescent probes to monitor the redox-state}

Some probes do not aim at specific analyte detection but rather monitor the general redox state that can transiently change over time (Table 2-9). These ratiometric sensors show a shift in fluorescence emission upon reaction with oxidants such as ROS, which can be reversed when more reducing conditions are applied, leading to a reversible ratiometric probe that indicates a specific redox state when the ratio of the two wavelengths is used. The group of E. New has summarized and critically evaluated the progress in the field of small organic molecule redox sensors ${ }^{[176,177]}$ and proposed ratiometric probes to sense oxidative stress. ${ }^{[178,179]}$ Flavin, a redox-responsive fluorescent dye, has been used as acceptor in a FRET dyad in combination with different donors. When combined with a coumarin donor (FCR1), a 6-fold reduction of the fluorescence emission ratio could be detected upon addition of a reducing agent (e.g. $\mathrm{NaBH}_{3} \mathrm{CN}$ ). Even though ratiometric imaging in $\mathrm{H}_{2} \mathrm{O}_{2}$-stimulated HeLa cells was shown to be possible with this combination, the probe's reversibility was not studied. ${ }^{[178]}$ When combined instead with rhodamine as donor (FRR2), the probe yielded a 3-4-fold increase in fluorescence emission ratio after reducing agents were applied, and showed good reversibility with up to 7 reduction and oxidation cycles. LPSstimulated macrophages showed a 1.7-fold increase, and the mitochondrial oxidative capacities of hematopoietic cells during different stages of embryonic development could be successfully monitored by fluorescence-activated cell sorting. ${ }^{[179]}$ These experiments show that small molecule probes can be successfully used to monitor transient changes in the redox state of cells. 
Table 2-9: Ratiometric fluorescent probes for the specific detection of ROS.

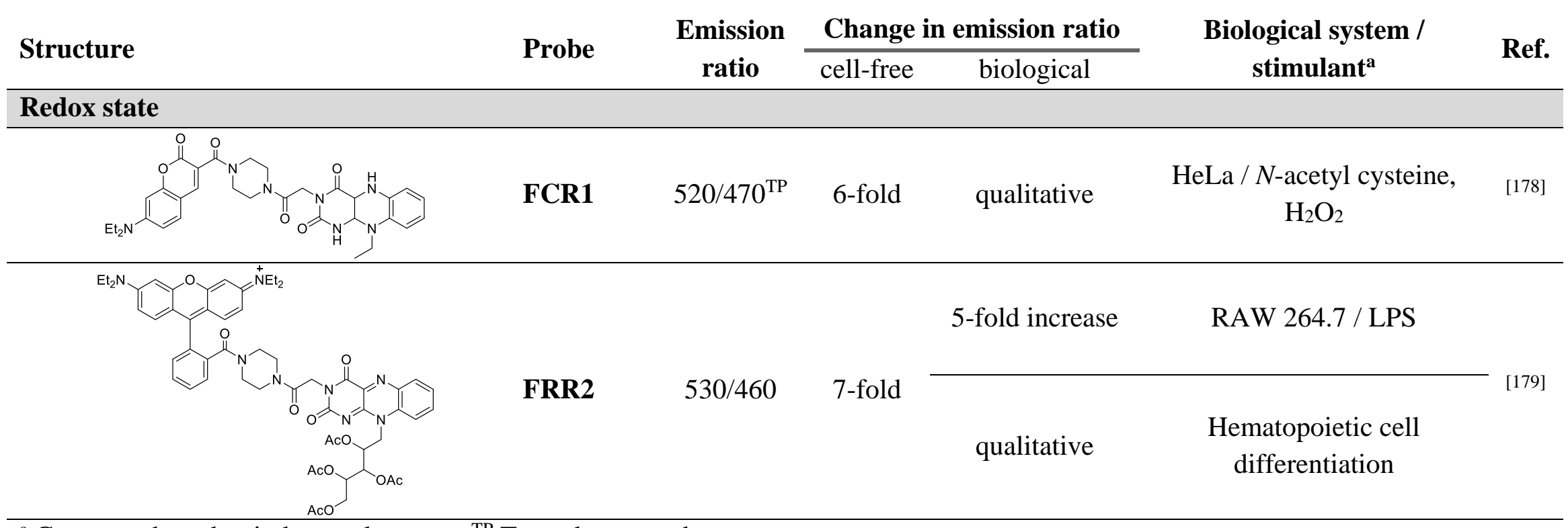

${ }^{\mathrm{a}}$ Compound used to induce redox state, ${ }^{\mathrm{TP}}$ Two-photon probe 


\subsection{Conclusion}

In the increasingly active field of ratiometric fluorescent sensors for ROS, new probes are constantly being introduced, usually with the aim of improving one or several features for a more reliable ROS detection (e.g. selectivity or sensitivity). Compared to the traditional and commercially available fluorophores, significant progress has been made with respect to selectivity and sensitivity. Nevertheless, ratiometric measurements are still inferior to intensitybased sensors, as the obtained detection limits are usually higher and specificity is often lower compared to turn-on probes. The fact that the introduction of new sensors has been favored over improving existing designs may explain their comparatively poor performance. The most promising systems are emission-ratiometric and are compatible with frequently used lasers lines (e.g. $488 \mathrm{~nm}, 640 \mathrm{~nm}$ or $750 \mathrm{~nm}$ ). Whereas innovation in small organic fluorophores has centered around creating new chemical structures, nano- and polymer-based sensors preferably use existing fluorophores and focus on implementing them in a novel scaffold. Although small organic molecules exhibit a lower toxicity on cells and allow to obtain a well-defined structure, solubility issues in aqueous media, fast bleaching and low retention times in cell compartments can rise concerns. Fluorescent nanoparticles exhibit higher photostability but their possible toxicity is a major issue. Small organic sensors are often grafted to the nanoparticles, which allows a high loading of the sensors but also complicates the characterization of the probes. Additionally, the sensors' translation from cell-free assays to more physiologically relevant conditions has repeatedly proven to be challenging. Frequently, the sensing capacity of these new probes was remarkably reduced as soon as they were tested in more complex biological matrix. To enable the study of biological samples also in vivo the differences in the biodistribution profiles of small molecules and nanoparticles should be considered during the design process. Firstly, the route of administration can greatly affect the fate of the system and should be carefully chosen. Whereas both types of scaffolds can be equipped with targeting units, the general accessibility of certain tissues (e.g. brain tissue) can be greatly reduced with nanoparticles. Another major challenge for nanoprobes and small molecules is the tuning of their pharmacokinetics. Overall, the real potential of many newly developed sensors remains to be established through further biological studies. Additionally, even though ratiometric probes eliminate the issues associated with fluctuations in fluorescence intensity, calibration curves are required to correlate the ratiometric signal to an analyte concentration. Therefore, the quantification of a specific ROS is understandably more complicated in in vivo settings than in 
cell culture applications, where feasibility has been demonstrated through several examples. In this regard, NIR sensors would greatly simplify in vivo applications, which are currently limited by the sub-optimal excitation and emission wavelengths used. To better understand the roles of ROS in biology, sensors with enhanced specificity, sensitivity and robustness are needed. The optimal design of a ratiometric probe may vary depending on the ROS of interest, that can be a single or multiple species. Even though promising sensing designs have been introduced for certain compounds, such as the use of boronate esters for the detection of $\mathrm{H}_{2} \mathrm{O}_{2}$, caution is still required as species are known that can interfere. In any case a systematic study of a specific scaffold should be greatly favored over the introduction of new designs to obtain a biologically well-tolerated sensor that allows for robust monitoring of ROS. While the newer generations of ratiometric fluorescent sensors have addressed some of these requirements, they have not yet found wide-spread use in general biological studies. This may be partly due to the limited availability of the newer probes, but also low solubility and aggregation of the sensor as well as difficulties in obtaining sufficient amounts limit their use in biological settings. 
Chapter 3

Hydrocyanines 


\subsection{Introduction}

Hydrocyanines are non-fluorescent sensors that can switch-on upon reaction with $\mathrm{O}_{2}{ }^{-}$and $\mathrm{OH}^{*}$ but not with $\mathrm{H}_{2} \mathrm{O}_{2}$. ${ }^{[40,180]}$ Sodium borohydride $\left(\mathrm{NaBH}_{4}\right)$ converts any cyanine dye into its corresponding hydrocyanine by reducing a double bond of the polymethine chain. The delocalized $\pi$-system can be restored upon reaction with ROS leading to a fluorescent turn-on response (Figure 3-1). Cyanine dyes are not only available with various reactive groups (e.g. NHS ester and maleimide) that allow to label biomolecules, polymers or nanocarriers, ${ }^{[181]}$ but also cover a wide range of emission wavelengths, namely from $500 \mathrm{~nm}$ to $810 \mathrm{~nm}$. These favorable characteristics and the ease of synthesis of hydrocyanines from cyanine dyes make this class of sensors a promising tool to detect ROS. Hydrocyanines were used in various biological systems such as imaging of LPS- and implant-induced inflammation in mice or detection of retinal oxidative stress. ${ }^{[40,182-189]}$ Additionally, modification of hydrocyanines for in vivo imaging (derivatizing with PEG or coupling them to a nanocarrier) were reported. ${ }^{[190,191]}$ Our choice to use hydrocyanines for ROS sensing was based on these promising reports.

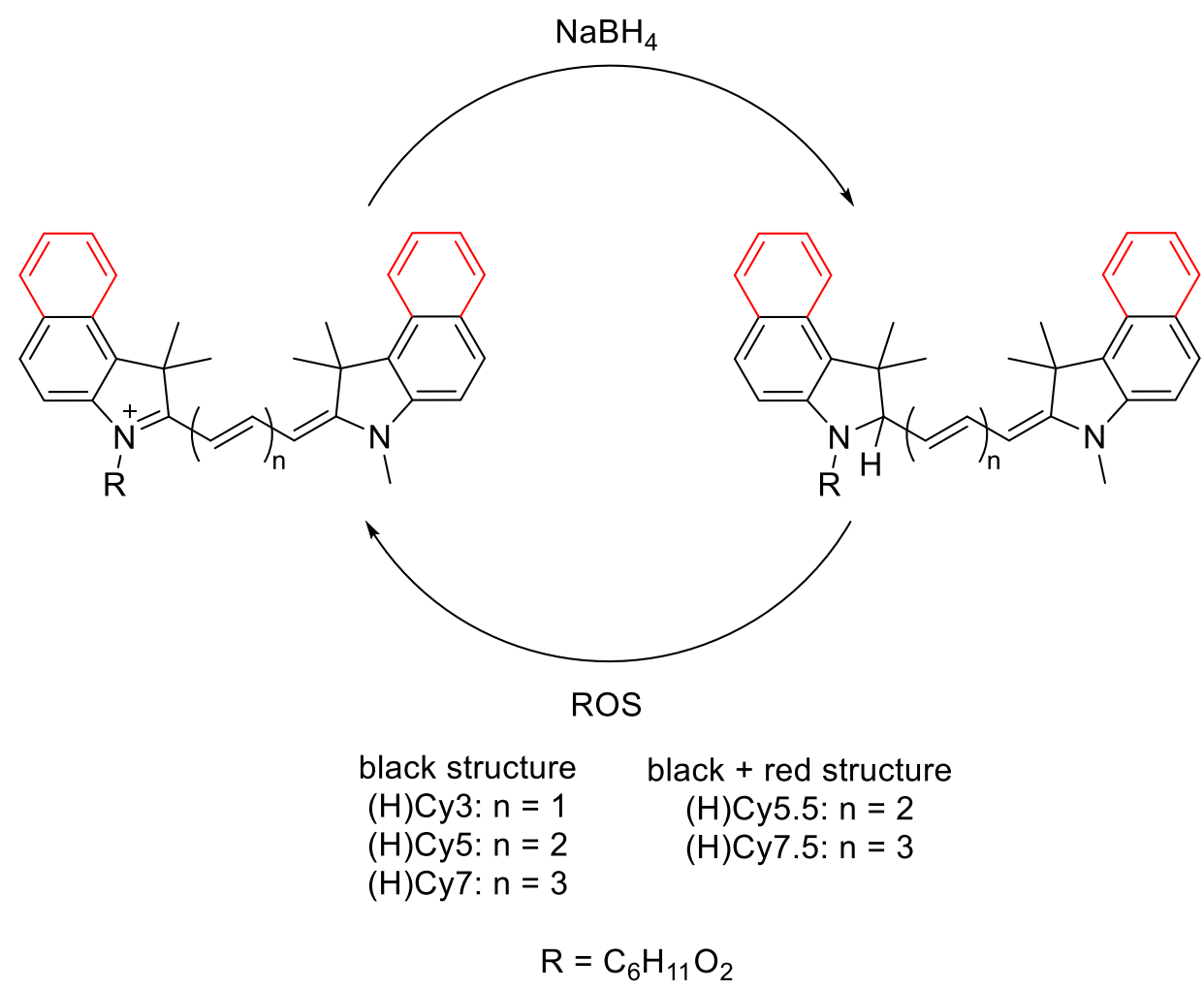

Figure 3-1: Reduction and re-oxidation cycle of cyanines and hydrocyanines, respectively, and nomenclature of (hydro)cyanine $(\mathrm{H}) \mathrm{Cy}$. 
First, we tested the sensing behavior of seven different hydrocyanines towards $\mathrm{HO}^{\circ}$, and selected hydrocyanine5 (HCy5) as model fluorophore due to its promising turn-on response and its stability toward hROS. The sensing capacity of $\mathrm{HCy} 5$ towards $\mathrm{O}_{2}{ }^{*-}$ and $\mathrm{HO}{ }^{\bullet}$ in aqueous media and in three cell-based assays was investigated. A stress response in cells and concomitant ROS production was stimulated with two different strategies: a) physical stress was applied to a cell monolayer and the stress response was monitored with HCy5 by fluorescent microscopy, b) the addition of chemical stimulants was used to induce elevated ROS levels in two cell models. An epithelial cell line (Caco-2) was challenged to mimic a stress response from intestinal epithelial tissue and the oxidative burst was triggered in human neutrophils to simulate an immune response in the intestine upon microbial invasion. Having evaluated the capacity of ROS detection with HCy5 in project-relevant in vitro models, the sensor was modified to minimize internalization by cells. The fluorophore was coupled to a long PEG molecule or to a highly charged poly(glutamic acid), and the uptake in differentiated Caco-2 monolayers was assessed by fluorescence activated cell sorting (FACS). 


\subsection{Experimental Section}

\subsubsection{Materials and Instruments}

Acetic acid $(\mathrm{AcOH})$, albumin (BSA, from bovine serum, $\geq 96 \%$ ), acetonitrile (ACN), 2,2azobis(2-methyl-propionamidine) dihydrochloride (AAPH), chloroform $\left(\mathrm{CHCl}_{3}\right)$, dichloromethane (DCM), N,N-diisopropylethylamine (DIPEA), dimethylformamide (DMF), diethylether $\left(\mathrm{Et}_{2} \mathrm{O}\right)$, ethylacetate (EtOAc), iron(II) sulfate $\left(\mathrm{FeSO}_{4}\right.$, heptahydrate), hydrochloric acid ( $\mathrm{HCl}, 37 \mathrm{wt} \%$ aq.), potassium iodide (KI), methyliodide (MeI), methanol (MeOH), sodium sulfate $\left(\mathrm{Na}_{2} \mathrm{SO}_{4}\right.$, anhydrous), sodium borohydride $\left(\mathrm{NaBH}_{4}\right)$, sodium chloride $(\mathrm{NaCl})$, piperidine, pyridine (py, over molecular sieves), silica gel for flash column chromatography (SilicaFlash F60, 230-400 mesh), trifluoroacetic acid (TFA), 2',7'-dichlorofluorescein diacetate (DCFHDA), MeO-PEG(20k)-NH2, phorbol 12-myristate 13-acetate (PMA) and Sephadex G25 ${ }^{\circledR}$ were purchased from Sigma Aldrich (Buchs, Switzerland). 1[bis(dimethylamino)methylene]-1H-1,2,3-triazolo[4,5-b]pyridinium-3-oxide hexafluorophosphate (HATU) and dimethyl sulfoxide (DMSO, 99.8\%, extra dry over molecular sieves) were obtained from Acros Organics (Geel, Belgium). 2,3,3-trimethylindolenine was purchased from TCI (Eschborn, Germany). 6-bromohexanoic acid and malondialdehyde bis(phenylimine) monohydrochloride were obtained from ABCR (Karlsruhe, Germany). Di-(4-carboxybenzyl) hyponitrite (SOTS-1) was bought from Cayman Chemical (Ann Arbor, MI). Acetic anhydride $\left(\mathrm{Ac}_{2} \mathrm{O}\right)$ and hydrogen peroxide $\left(\mathrm{H}_{2} \mathrm{O}_{2}\right)$ were obtained from Merck (Darmstadt, Germany). Fmoc-Glu( $\left.{ }^{t} \mathrm{Bu}\right)-\mathrm{OH}$ and Fmoc-Lys(Boc)-OH were purchased from Merck Millipore (Billerica, MA). Deuterated dimethyl sulfoxide (DMSO-d6) was obtained from Cambridge Isotope Laboratories Inc. (Tewksbury, MA). Caco-2 cells were kindly provided by Dr. Vanessa LeMoal (Université Paris-Sud). Dulbecco's Modified Eagle Medium (DMEM) with GlutaMAX $^{\mathrm{TM}}$ (high glucose), penicillin/streptomycin mixture, fetal bovine serum (FBS), phosphate buffered saline (PBS), 0.25\% trypsin/EDTA, non-essential amino acids, Hank's balanced salt solution (HBSS), ACK lysing buffer were obtained from ThermoFisher Scientific (Waltham, MA). Cy3, Cy5, sulfo-Cy5, Cy5.5, Cy7 and Cy7.5 were purchased from Lumiprobe (Hannover, Germany). Dihydrorhodamine 123 (DHR123) was from Chemodex (St. Gallen, Switzerland). IRDye680 was obtained from Li-Cor Biosciences (Lincoln, NE). Fresh blood was received from Blutspende Zürich (Schlieren, Switzerland). Ficoll-Paque PLUS was obtained from GE Healthcare Life Sciences (Glattbrugg, Switzerland), MycoAlert ${ }^{\mathrm{TM}}$ detection kit was purchased from Lonza (Visp, Switzerland). 
XBridge $^{\mathrm{TM}} \mathrm{C} 18$ column $(5 \mu \mathrm{m}, 4.6 \times 250 \mathrm{~mm})$ was used from Waters Peptide Separation Technology (Baden-Dättwil, Switzerland). LaChrom Classic HPLC L-7200 and TLC plates Silica gel 60 F254 were provided by Merck (Zug, Switzerland) and UltiMate 3000 LC System (Dionex) by Thermo Fisher Scientific (Reinach, Switzerland). Samples were concentrated in vacuo on the Rotavapor R-210 and heating bath B-491 by Büchi (Uster, Switzerland). ${ }^{1} \mathrm{H}-\mathrm{NMR}$ and ${ }^{13} \mathrm{C}-\mathrm{NMR}$ spectra were recorded on Bruker AV 400. Chemical shifts are expressed in parts per million (ppm) and coupling constants are reported in hertz $(\mathrm{Hz})$. Splitting patterns are indicated as follows: $b r$, broad; $s$, singlet; $d$, doublet; $d d$, doublet of doublet; $t$, triplet; $q$, quartet; $m$, multiplet. High resolution mass spectrometry (HRMS)-ESI was recorded on Bruker solariX by the MS service of ETHZ. Solid phase peptide synthesis (SPPS) was implemented on the shaker KS125 basic by IKA Labortechnik (Staufen, Germany) in a peptide synthesis reactor 2.5 or $5 \mathrm{~mL}$ and frit SF-0500 by Torviq (Niles, MI). The plate reader infiniteM200 with Costar96 flat black well plates were provided by Tecan (Männedorf, Switzerland). CHRIST ${ }^{\circledR}$ Lyophilisator from Kuhner (Birsfelden, Switzerland), Varian Cary300 Bio UV-VIS Spectrophotometer (now Agilent, Basel, Switzerland) was used. FACS analysis was carried out on a FACSCanto ${ }^{\mathrm{TM}}$ Flow Cytometer (BD Biosciences, San Jose, CA).

\subsubsection{Synthesis of Cy5}

\section{1,2,3,3-Tetramethyl-3H-indol-1-ium iodide 1}

2,3,3-Trimethylindolenine (2.02 mL, $12.5 \mathrm{mmol}, 1 \mathrm{eq})$ was diluted in $10 \mathrm{~mL}$ ACN. MeI $(0.86 \mathrm{~mL}, 13.8 \mathrm{mmol}, 1.1 \mathrm{eq})$ was slowly added and the reaction mixture was refluxed at $85^{\circ} \mathrm{C}$ for $16 \mathrm{~h}$. The purple solid was filtered and washed with $\mathrm{Et}_{2} \mathrm{O}$ and DCM. A pink powder was obtained (3.38 g, $11.2 \mathrm{mmol}, 90 \%$ yield).

${ }^{1} \mathbf{H}$ NMR (400 MHz, DMSO): $\delta=7.97$ - 7.88 (m, 1H), 7.88 - $7.79(\mathrm{~m}, 1 \mathrm{H}), 7.69-7.57$ (m, 2H), 3.98 (s, 3H), 2.77 (s, 3H), 1.53 (s, 6H); ${ }^{13}$ C NMR (101 MHz, DMSO): $\delta=195.82,141.81$, 141.37, 129.08, 128.58, 123.06, 114.89, 53.69, 34.47, 21.46, 13.90; HRMS-ESI calculated for $[\mathrm{C} 12 \mathrm{H} 16 \mathrm{~N}] \mathrm{m} / \mathrm{z}=174.1277$, found: $m / z=174.1273$. The characterization data are in agreement with those previously reported (Figure A1-2). ${ }^{[192]}$

\section{1-(5-Carboxypentyl)-2,3,3-trimethyl-3H-indol-1-ium iodide 2}

2,3,3-Trimethylindolenine (1.6 mL, $10 \mathrm{mmol}, 1 \mathrm{eq})$ and 6-bromohexanoic acid (1.95 g, 10 mmol, 1 eq) were dissolved in $6 \mathrm{~mL} \mathrm{ACN}$. KI (1.66 g, $10 \mathrm{mmol}, 1 \mathrm{eq})$ was added and the reaction mixture was refluxed at $85{ }^{\circ} \mathrm{C}$ for $16 \mathrm{~h}$. The liquid was decanted and solid $\mathrm{KBr}$ was 
removed. The organic layer was concentrated under reduced pressure and precipitated in an EtOAc/DCM mixture. A pale pink powder was obtained (2.37 g, $5.9 \mathrm{mmol}, 59 \%$ yield).

${ }^{1}$ H NMR (400 MHz, DMSO): $\delta=12.00$ (s, 1H), 8.00 - 7.93 (m, 1H), 7.86 - $7.80(\mathrm{~m}, 1 \mathrm{H}), 7.71$ - $7.56(\mathrm{~m}, 2 \mathrm{H}), 4.44(\mathrm{t}, J=7.7 \mathrm{~Hz}, 2 \mathrm{H}), 2.83(\mathrm{~s}, 3 \mathrm{H}), 2.22$ (t, $J=7.2 \mathrm{~Hz}, 2 \mathrm{H}), 1.84(\mathrm{~m}, 2 \mathrm{H})$, $1.63-1.49$ (m, 8H), 1.48 - 1.37 (m, 2H); ${ }^{13}$ C NMR (101 MHz, DMSO): $\delta=196.50,174.31$, $141.87,141.05,129.42$, 128.95, 123.51, 115.48, 54.16, 47.41, 33.35, 26.94, 25.42, 24.01, 22.01, 13.96 HRMS-ESI calculated for [C17H24N02] $\mathrm{m} / z=274.1802$, found: $\mathrm{m} / z=274.1800$. The characterization data are in agreement with those previously reported (Figure A3-4). ${ }^{\text {192] }}$

\section{Cyanine $5(C y 5,3)$}

2 (648 mg, $1.6 \mathrm{mmol}, 1 \mathrm{eq}$ ) and malondialdehyde bis(phenylimine) monohydrochloride (556 $\mathrm{mg}, 2.5 \mathrm{mmol}, 1.6 \mathrm{eq}$ ) were dissolved in $5 \mathrm{~mL} \mathrm{Ac} 2 \mathrm{O}$ and the mixture was refluxed at $130{ }^{\circ} \mathrm{C}$ for $30 \mathrm{~min}$. Then 1 (873 mg, $2.9 \mathrm{mmol}, 1.8 \mathrm{eq}$ ) and $5 \mathrm{~mL}$ dry pyridine were added. The reaction was left stirring at room temperature for $16 \mathrm{~h}$. The solvents were removed under reduced pressure at $60{ }^{\circ} \mathrm{C}$. The blue solid was dissolved in DCM, washed with brine and $1 \mathrm{M} \mathrm{HCl}_{\mathrm{aq}}$. The combined organic layers were dried over $\mathrm{Na}_{2} \mathrm{SO}_{4}$ and removed under reduced pressure. The crude mixture was purified by silica column chromatography eluting with a gradient of $0-10 \% \mathrm{MeOH}$ in DCM. $394 \mathrm{mg}$ Cy5 3 was obtained (0.76 mmol, 48\% yield).

${ }^{1}$ H NMR (400 MHz, DMSO): $\delta=8.33(\mathrm{t}, J=13.1 \mathrm{~Hz}, 2 \mathrm{H}), 7.62(\mathrm{~d}, J=7.4 \mathrm{~Hz}, 2 \mathrm{H}), 7.47$ $7.33(\mathrm{~m}, 4 \mathrm{H}), 7.33-7.17(\mathrm{~m}, 2 \mathrm{H}), 6.57$ (t, $J=12.3 \mathrm{~Hz}, 1 \mathrm{H}), 6.29(\mathrm{dd}, J=17.9,13.9 \mathrm{~Hz}, 2 \mathrm{H})$, $4.09(\mathrm{t}, J=7.1 \mathrm{~Hz}, 2 \mathrm{H}), 3.60(\mathrm{~s}, 3 \mathrm{H}), 2.24-2.11(\mathrm{~m}, 2 \mathrm{H}), 1.74-1.61(\mathrm{~m}, 14 \mathrm{H}), 1.61-1.49$ (m, 2H), $1.46-1.31(\mathrm{~m}, 2 \mathrm{H}) .{ }^{13} \mathbf{C}$ NMR (101 MHz, DMSO): $\delta=173.25,172.50,154.00$, $142.76,142.02$, 141.08, 141.01, 128.42, 128.35, 125.33, 124.73, 124.62, 122.43, 122.30, $111.04,103.30,103.02,48.85,43.23,40.15,39.94,39.73,39.52,39.31,39.10,38.89,33.88$, 31.08, 27.17, 26.99, 26.70, 25.70, 24.33 HRMS-ESI calculated for [C32H39N202] $\mathrm{m} / \mathrm{z}=$ 483.3006, found: $m / z=483.3003$. The characterization data are in agreement with those previously reported (Figure A5-6). ${ }^{[192]}$ 


\subsubsection{Reduction of the cyanine dye}

To cyanine (Table 3-1) in $\mathrm{MeOH}$ a bubbling $\mathrm{NaBH}_{4}$ solution in $\mathrm{MeOH}(1 \mathrm{mg} / \mathrm{mL})$ was added. The loss of color indicated the reduction of Cy to HCy5.

Table 3-1: Fluorescence excitation and emission wavelength used in the screening of (hydro)cyanines tested.

\begin{tabular}{|l|l|l|}
\hline Dye & $\lambda_{\text {ex }}(\mathbf{n m})$ & $\lambda_{\text {em }}(\mathbf{n m})$ \\
\hline (H)Cy3 & 525 & 570 \\
\hline (H)Cy5 & 620 & 665 \\
\hline (H)sulfo-Cy5 & 620 & 665 \\
\hline (H)IRDye 680RD & 650 & 718 \\
\hline (H)Cy5.5 & 630 & 714 \\
\hline (H)Cy7.5 & 770 & 816 \\
\hline (H)Cy7 & 735 & 780 \\
\hline
\end{tabular}

\subsubsection{Re-oxidation with the Fenton's reagent - $\mathrm{HO}^{\circ}$ generation}

Screening of commercial hydrocyanines: Ten $\mu \mathrm{M}$ hydrocyanine in $\mathrm{MeOH}$ were mixed with freshly prepared $\mathrm{FeSO}_{4}$ stock solution $(1 \mathrm{mM})$ in $\mathrm{H}_{2} \mathrm{O}$ yielding a final concentration of 20 or $50 \mu \mathrm{M}$. Afterwards, $\mathrm{H}_{2} \mathrm{O}_{2}$ (stock solution: $10 \mathrm{mM}$ ) reaching a concentration of 200 or $500 \mu \mathrm{M}$, respectively, was added immediately. The fluorescence intensity was detected after $10 \mathrm{~min}$.

Re-oxidation in $\mathrm{H}_{2} \mathrm{O}$ : Ten $\mu \mathrm{M}$ imaging agent was mixed with freshly prepared $\mathrm{FeSO}_{4}$ solution in $\mathrm{H}_{2} \mathrm{O}$ yielding a final concentration of 20 or $50 \mu \mathrm{M}$. Afterwards, $\mathrm{H}_{2} \mathrm{O}_{2}$ reaching a concentration of 200 or $500 \mu \mathrm{M}$, respectively, was added immediately. The fluorescence intensity (Cy5 $\lambda_{\mathrm{ex}}=620 \mathrm{~nm}, \lambda_{\mathrm{em}}=665$, gain 200, DHR $\lambda_{\mathrm{ex}}=500 \mathrm{~nm}, \lambda_{\mathrm{em}}=535$, gain 100) was monitored every 5 min over $2 \mathrm{~h}$ at $37{ }^{\circ} \mathrm{C}$ on a plate reader in a black multi-well quartz plate (Hellma Analytics) equipped with a sealed quartz cover. All experiments were carried out in triplicate and independently repeated 3 times. 


\subsubsection{Re-oxidation with SOTS-1 $-\mathbf{O}_{2}{ }^{*-}$ generation $^{[193,194]}$}

Ten $\mu \mathrm{M}$ imaging agent were mixed with SOTS- 1 ( $1 \mathrm{mg} / \mathrm{mL}$ in DMF) in $\mathrm{H}_{2} \mathrm{O}$ (ratio DMF: $\mathrm{H}_{2} \mathrm{O}$ $1: 2, v / v)$ to generate up to $120 \mu \mathrm{M} \mathrm{O}_{2}{ }^{\circ}$. $\mathrm{O}_{2}{ }^{*}$ generation follows a first order kinetic and is produced in $40 \mathrm{~mol} \%$ according to literature. ${ }^{[193,194]}$ The indicated concentration corresponds to the maximal possible production of $\mathrm{O}_{2}{ }^{-}$but might likely be lower in reality. The fluorescence intensity (Cy5 $\lambda_{\mathrm{ex}}=620 \mathrm{~nm}, \lambda_{\mathrm{em}}=665$, gain 150 , DHR $\lambda_{\mathrm{ex}}=500 \mathrm{~nm}, \lambda_{\mathrm{em}}=535$, gain 100) was monitored as stated above.

\subsubsection{Cell culture}

Caco-2 cells were maintained in DMEM with GlutMAX ${ }^{\mathrm{TM}}$ (high glucose) supplemented with 15 vol\% FBS, 1 vol\% penicillin/streptomycin mixture (penicillin: 10,000 units/mL, streptomycin: $10,000 \mu \mathrm{g} / \mathrm{mL}$ ) and 1 vol\% non-essential amino acids at $37{ }^{\circ} \mathrm{C}$ in a $5 \% \mathrm{CO}_{2}$ humidified atmosphere. The cells were used from passage 68 to 90 . The cells were routinely tested negative for mycoplasma with the MycoAlert ${ }^{\mathrm{TM}}$ Dection Kit.

\subsubsection{TEER measurement of differentiated Caco-2 cells}

Caco-2 cells were seeded at 200,000 cell/mL in Corning transwell ${ }^{\mathrm{TM}}$ insert $(0.4 \mu \mathrm{m}, 12 \mathrm{~mm}$ diameter, $0.5 \mathrm{~mL}$ apical side, $1.5 \mathrm{~mL}$ basolateral side) and grown for 21 days. The transepithelial electrical resistance (TEER) was measured every 2-3 days using an EVOM epithelial voltohmmeter (World Precision Instruments) equipped with "chopstick" electrodes. The electrodes were incubated in Caco-2 growth medium for one hour before measurements. Resistance measurements were taken every 2-3 days for 3 weeks.

\subsubsection{Histology of differentiated Caco-2 cells}

Differentiated Caco-2 cells were grown in Corning transwell ${ }^{\mathrm{TM}}$ insert for 14 days as stated above. Then, growth medium was removed and the cells were fixed with Methacarn $(60 \%$ $\mathrm{MeOH}, 30 \%$ chloroform, $10 \%$ acetic acid) for $60 \mathrm{~min}$. After washing the insert with PBS, the membrane was cut out from the transwell with a scalpel and was transferred to $70 \% \mathrm{EtOH}$ in water. The cell membranes were dehydrated through a series of alcohol washing steps and embedded in paraffin wax. Slices of $10 \mu \mathrm{m}$ were cut and mounted on glass slides. The 
hematoxylin and eosin staining was carried out using a routine staining procedure on a COT 20 (Medite AG) automated instrument.

\subsubsection{Scratch-wounding assay with differentiated Caco-2 cells}

Cells were grown in a 6-well plate for 15 days, so that the complete bottom of the well was covered with a differentiated Caco-2 monolayer. Wells were washed with PBS buffer and the wounding was carried out as one scratch using a glass pipette connected to the aspirator and the PBS was aspired at the same time. A $20-\mu \mathrm{M}$ solution of HCy5 was added, and fluorescence images were recorded with a Leica DMI6000B microscope equipped with an EL6000 Hg light source every $5 \mathrm{~min}$ for $65 \mathrm{~min}$.

\subsubsection{Stimulation of non-differentiated Caco-2 cells}

Caco- 2 cells were seeded at a seeding density of 50,000 cells/well on 96-well microtiter plates. After $24 \mathrm{~h}$, the growth medium was removed, and the cells were washed with $100 \mu \mathrm{L}$ PBS, followed by incubation with either a solution without stimulant, a 1-mM solution of AAPH or a 1-mM solution of $\mathrm{H}_{2} \mathrm{O}_{2}$ all of them containing $10 \mu \mathrm{M}$ of HCy5 or DHR. DCFHDA was preincubated with the cells in advance for $30 \mathrm{~min}$. The fluorescence intensity was monitored with a plate reader at $37^{\circ} \mathrm{C}$ for $2 \mathrm{~h}$. HCy5: $\lambda_{\mathrm{ex}}=620 \mathrm{~nm}, \lambda_{\mathrm{em}}=665 \mathrm{~nm}$, gain: 200 , DCFHDA: $\lambda_{\mathrm{ex}}=$ $485 \mathrm{~nm}, \lambda_{\mathrm{em}}=530 \mathrm{~nm}$, gain: 80; DHR: $\lambda_{\mathrm{ex}}=490 \mathrm{~nm}, \lambda_{\mathrm{em}}=534 \mathrm{~nm}$, gain: 80 .

\subsubsection{Stimulation of human neutrophils}

Human neutrophils were isolated from fresh blood samples obtained from Blutspende Zurich. The blood was diluted 1:2( $v / v)$ with HBSS. Granulocytes and erythrocytes were separated from plasma, lymphocytes, monocytes and platelets with a density gradient. The diluted blood sample was slowly added on top of a Ficoll-Paque PLUS layer and was centrifuged to separate the different components $\left(300 \times g, 4{ }^{\circ} \mathrm{C}\right.$, slow acceleration). Erythrocytes were lysed with ACK buffer (ratio $1: 6 \mathrm{v} / \mathrm{v}$ ) for $30 \mathrm{~min}$ on ice. After centrifugation $\left(280 \times g, 4{ }^{\circ} \mathrm{C}, 5 \mathrm{~min}\right)$, the pellet was re-suspended and washed with PBS. The procedure was repeated until the pellet was white/grey, indicating that the lysis of erythrocyte was complete. The white/grey pellet was resuspended in FACS buffer (PBS with 2 mM EDTA and 0.5\% BSA) and were used within $3 \mathrm{~h}$. Neutrophils were incubated with DCFHDA $(10 \mu \mathrm{M})$, DHR $(2 \mu \mathrm{M})$ or HCy5 $(20 \mu \mathrm{M})$ at $37^{\circ} \mathrm{C}$ 
for $30 \mathrm{~min}$. Then cells were washed with FACS buffer twice. PMA (200 nM) was added to stimulate neutrophil and fluorescence intensity was monitored with a plate reader at $37{ }^{\circ} \mathrm{C}$ for 2 h. HCy5: $\lambda_{\mathrm{ex}}=620 \mathrm{~nm}, \lambda_{\mathrm{em}}=665 \mathrm{~nm}$, gain: 200, DHR and DCFH: $\lambda_{\mathrm{ex}}=485 \mathrm{~nm}, \lambda_{\mathrm{em}}=530$ nm, gain: 150 .

\subsubsection{PEGylation of cyanines}

Cy5-NHS was mixed with an equimolar amount of MeO-PEG(20k)- $\mathrm{NH}_{2}$ in dry DMSO and the reaction mixture was agitated at $800 \mathrm{rpm}$ for $6 \mathrm{~h}$ at $30{ }^{\circ} \mathrm{C}$. Ten-fold excess of $\mathrm{H}_{2} \mathrm{O}$ was added, and the crude was lyophilized. Then the reaction was reconstituted in $\mathrm{H}_{2} \mathrm{O}$ and purified with a Sephadex G25 ${ }^{\circledR}$ size exclusion column. The absence of free Cy5 was verified by HPLC with an isocratic gradient of MeOH/PBS (80:20 $v / v)$ and a C18 end capped column (Figure A7).

\subsubsection{Quantification of modified cyanine dye}

Cy5 conjugates were quantified with a UV spectrophotometer. Absorbance of Cy5 $\left(\lambda_{\mathrm{abs}, \max }=\right.$ $649 \mathrm{~nm}$ ) was measured in $\mathrm{MeOH}$ and the concentration c was calculated using Equation 3-1, with $\mathrm{A}_{\text {dye,max }}$ being the absorption at $\lambda_{\text {abs,max }}$ and $\varepsilon_{\text {dye }}$ the extinction coefficient of the dye.

$$
c_{\text {cyanine-conjugate }}=\frac{A_{\text {dye, } \text { max }}}{\varepsilon_{\text {dye }} \times \text { path legnths }}
$$

Equation 3-1

\subsubsection{Synthesis of Cy5-Glug-Lys}

SPPS was performed manually in a poly(propylene) syringe $(2.5$ and $5 \mathrm{~mL}$ ) equipped with a frit at the bottom. All reactions were carried out on a vertical shaker at $600-800 \mathrm{rpm}$ at room temperature. Rink amide resin was swollen in DCM for $30 \mathrm{~min}$, and washed 5 times with DMF using 3 resin volumes. Fmoc was triple de-protected ( $1 \mathrm{~min}, 3 \mathrm{~min}, 15 \mathrm{~min}$ ) with piperidine in DMF $(20 \% v / v)$ using one resin volume and then washed 5 times with DMF using 3 resin volumes. Fmoc-Lys(Boc)-OH (5 eq) was pre-activated with HATU (4.9 eq) in DMF for 10 min. Then DIPEA (10 eq) was added and the mixture was transferred to the syringe and shaken for 60 min. The resin was washed 5 times with DMF using 3 resin volumes. This procedure was repeated 9 times for Fmoc-Glu( $\left.{ }^{t} \mathrm{Bu}\right)-\mathrm{OH}$ leading to resin-Lys(Boc)-Glu( $\left.{ }^{t} \mathrm{Bu}\right) 9-\mathrm{Fmoc}$. After Fmoc deprotection, Cy5-OH (2.5 eq, 3) was activated with HATU (2.4 eq) in DMF for 10 min. 
DIPEA (5 eq) was added and the mixture was transferred to the syringe and shaken for $90 \mathrm{~min}$. The resin was washed 5 times with DMF and 5 times with DCM using 3 resin volumes and was dried in vacuo for $16 \mathrm{~h}$. The peptide was double-cleaved from the resin using a mixture of TFA: $\mathrm{H}_{2} \mathrm{O}$ :TIS $(v / v, 95: 2.5: 2.5)$ for $60 \mathrm{~min}$. Lys-PGA-Cy5 was precipitated in ice-cold $\mathrm{Et}_{2} \mathrm{O}$ and centrifuged at $4000 \times g$ for 10 min at $4{ }^{\circ} \mathrm{C}$. The blue pellet was dissolved in $\mathrm{H}_{2} \mathrm{O}$ and lyophilized twice. The sample was analyzed by analytical HPLC and MS analysis (Figure A8-9).

\subsubsection{Uptake in Caco-2 cells}

Caco- 2 cells were seeded in 12-well plate (66,000 cells/well) and grown for 14 days. Medium was exchanged every other day and the day before the experiment. Cells were washed with $1 \mathrm{~mL}$ pre-warmed PBS and trypsinized with $0.3 \mathrm{~mL}$ of $0.25 \%$ trypsin for $15 \mathrm{~min}$ at $37^{\circ} \mathrm{C}$. Cells were counted and 250,000 cells/mL and sample were used in the uptake experiment. After centrifugation at $300 \mathrm{xg}$ for $5 \mathrm{~min}$ at $4{ }^{\circ} \mathrm{C}$, cells were washed once with FACS buffer (PBS supplemented with $0.5 \% \mathrm{w} / \mathrm{v}$ BSA and $2 \mathrm{mM}$ EDTA) and were then resuspended in FACS buffer containing $5 \mu \mathrm{M}$ of Cy5 or Cy5-PEG or Cy5-PGA. The samples were incubated for $15 \mathrm{~min}$ at $37^{\circ} \mathrm{C}$ in the dark. Cells were centrifuged $\left(300 \times \mathrm{g}, 5 \mathrm{~min}, 4^{\circ} \mathrm{C}\right)$ and washed once with FACS buffer, twice with an acid wash $(0.2 \mathrm{M} \text { acetic acid, } 0.5 \mathrm{M} \mathrm{NaCl})^{[195]}$ and once with FACS buffer. Flow cytometry measurements (FACSCanto ${ }^{\mathrm{TM}}$ Flow Cytometer) were performed in FACS buffer. A minimum of 10,000 events per sample were recorded. The 633-nm laser was used for the Cy5 excitation and the APC channel to detect the fluorescence emission. All experiments were carried out in triplicates and three independent experiments. 


\subsection{Results and Discussion}

\subsubsection{Screening of different commercial cyanine dyes with Fenton's reagent}

To test the turn-on response of different hydrocyanines, commercially available cyanine dyes were reduced with $\mathrm{NaBH}_{4}$ in $\mathrm{MeOH}$. The loss of color and fluorescence intensity indicated a successful reduction. The re-oxidation was carried out in $\mathrm{MeOH}$ by adding Fenton's reagent ( $\mathrm{FeSO}_{4}$ and $\mathrm{H}_{2} \mathrm{O}_{2}$ ) at two different concentrations, and the fluorescence intensity measured after 10 min was normalized to the sample containing the reduced dye but no Fenton's reagent Equation 3-2.

Norm. fluorescence intensity $=\frac{\text { Fluorescence intensity of hydrocyanine }+ \text { Fenton }}{\text { Fluorescence intensity of hydrocyanine }}$ Equation 3-2 In this screening, the turn-on responses for $\mathrm{HCy} 5, \mathrm{HCy} 5.5$ and $\mathrm{HCy} 7$ were similar with a 5- to 7-fold increase for the lower $\mathrm{OH}^{*}$ concentration and an 8- to 11- fold increase for the higher $\mathrm{OH}^{*}$ concentration (Figure 3-2). HCy3 exhibited a 3- and 7-fold increase in signal intensity, respectively. These four sensors showed a concentration dependent behavior, which is in contrast to the turn-on response of $\mathrm{HCy} 7.5$ that was lower (2.5-fold) and similar for both $\mathrm{OH}^{*}$ concentrations. The fluorescence intensity increase of dyes containing sulfates (sulfoCy5 and IRDye680) was small and no concentration dependent increase was detected. Generally, the observed effect was smaller than expected from literature. ${ }^{[40,180]} \mathrm{Cy} 5 / \mathrm{HCy} 5$ was used for all further studies due to its promising turn-on response and the presumably higher stability compared to $\mathrm{Cy} 7 / \mathrm{HCy} 7 .{ }^{[126]}$

\subsubsection{Synthesis of hydrocyanine5 (HCy5)}

The selected fluorophore Cy5 was synthesized according to previous reports in literature (Figure 3-3). ${ }^{[192]}$ 2,3,3-Trimethylindolenine was alkylated with methyl iodide or 6bromohexanoic acid in acetonitrile resulting in compounds $\mathbf{1}$ and $\mathbf{2}$. The powders were obtained in good yields (90\% and 59\%, respectively) and analyzed by ${ }^{1} \mathrm{H}-{ }^{13} \mathrm{C}-\mathrm{NMR}$. The data corresponded to accounts in literature (Figure A1-4). ${ }^{[192]}$

2 was refluxed with malondialdehyde bis(phenylimine) in acetic anhydride for $30 \mathrm{~min}$ and, cooled down to room temperature. Pyridine and $\mathbf{1}$ were added to the reaction mixture and the suspension was stirred for $15 \mathrm{~h}$. The dark blue crude mixture was dried under reduced pressure and purified by a silica gel column using a gradient of $0-10 \% \mathrm{MeOH}$ in DCM. Cy5 3 was 
obtained in $30-48 \%$ yield as blue solid and was characterized by ${ }^{1} \mathrm{H}-,{ }^{13} \mathrm{C}-\mathrm{NMR}$ and spectrophotometric methods (Figure A5-6). The reduction of Cy5 was carried out in $\mathrm{MeOH}$ analogous to the commercial dyes in section 3.3.1.

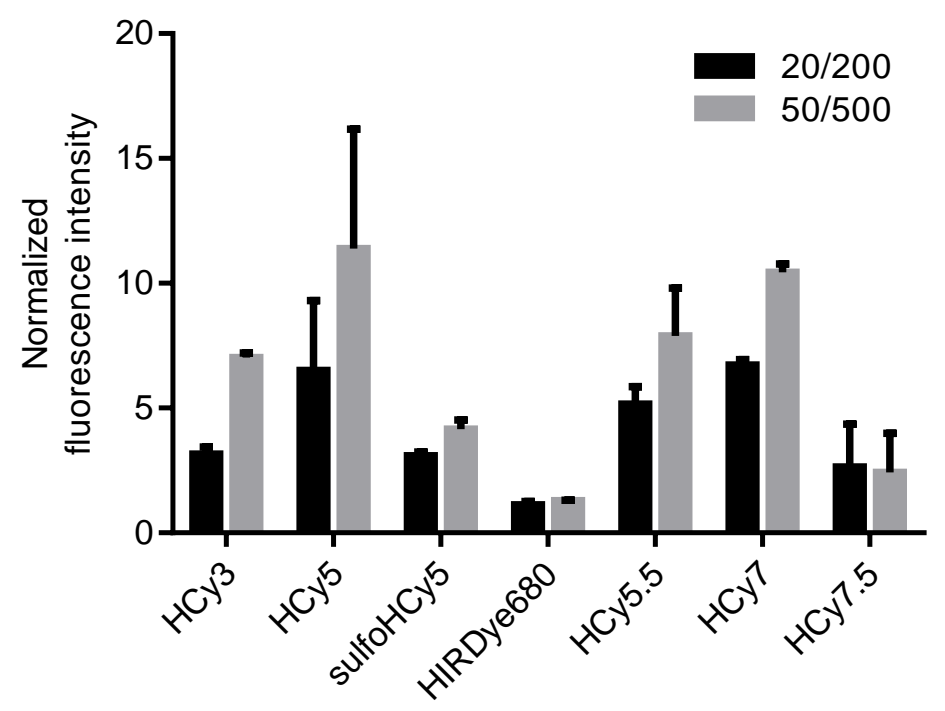

Figure 3-2: Oxidation with $\mathrm{OH}^{\bullet}$ generated by Fenton's reagent of hydrocyanines synthesized from commercially available cyanine dyes. Legend: $\left[\mathrm{FeSO}_{4}\right] /\left[\mathrm{H}_{2} \mathrm{O}_{2}\right]$ in $\mu \mathrm{M}$. $($ Mean $+\mathrm{SD}, \mathrm{n}=3-10)$.

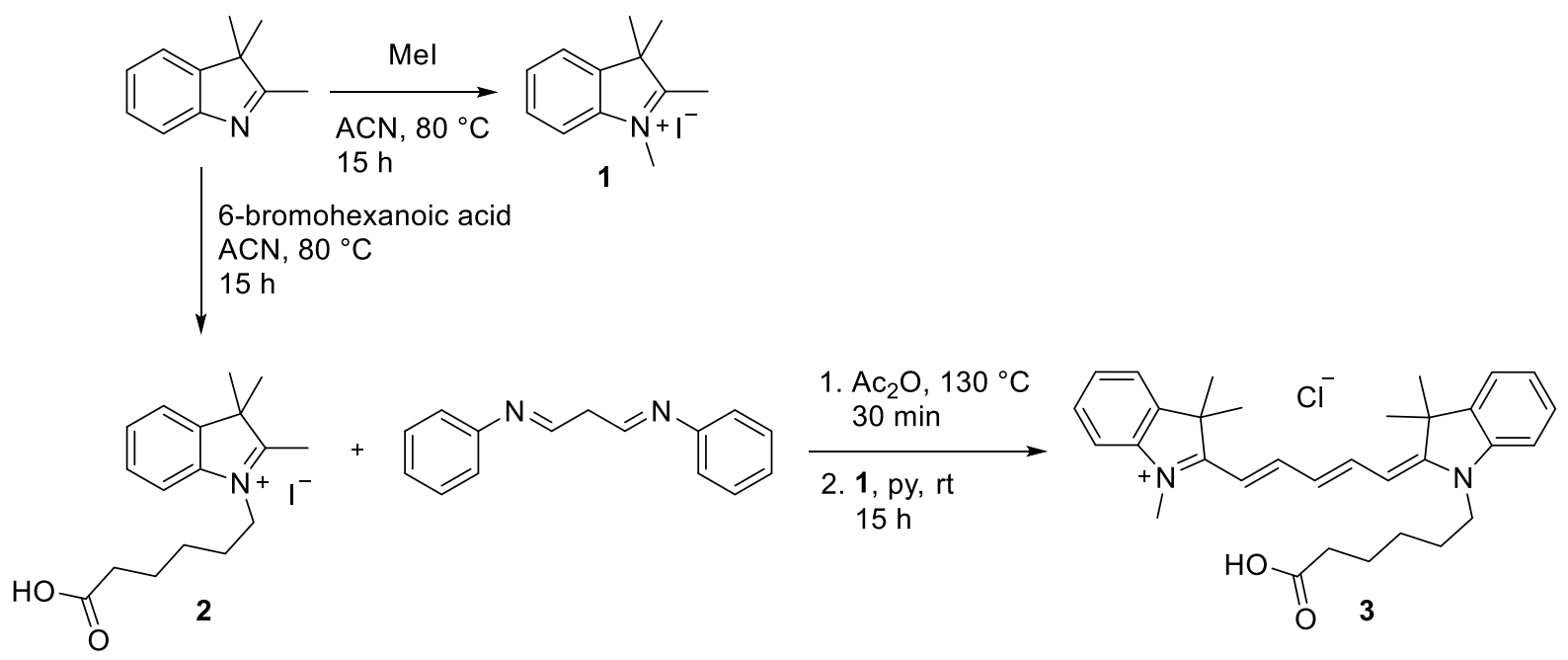

Figure 3-3: Synthetic scheme of the synthesis of Cy5. 


\subsubsection{Sensing of $\mathrm{OH}^{\bullet}$ and $\mathrm{O}_{2}{ }^{*-}$}

Sensing experiments were carried out with the freshly synthesized $\mathrm{HCy} 5 . \mathrm{OH}^{*}$ detection was repeated in an optimized set-up, where the turn-on response of the dye was monitored over $2 \mathrm{~h}$ in water in a quartz plate with a cover. The re-oxidation with the Fenton's reaction reached its maximum after approximately 20 - $30 \mathrm{~min}$ and revealed a 30- and 45-fold increase in fluorescence intensity (Figure 3-4 a). For the higher concentration $(50 / 500 \mu \mathrm{M})$ an overoxidation was observed, which led to a loss of fluorescence intensity after $30 \mathrm{~min}$ (Figure 3-4 b). ${ }^{[126]}$ The turn-on response was stronger compared to the commercial Cy5, and might be due to a higher purity of the freshly synthesized fluorophore. The re-oxidation capacity in this experiment corresponded to the one reported by Kundu et al. but could only be reproduced to such an extend after extensive optimization of the published protocol and very stringent experimental settings.

$\mathrm{O}_{2}{ }^{\bullet-}$ detection was investigated by using a $\mathrm{O}_{2}{ }^{\bullet-}$ generator (SOTS-1). ${ }^{[193,194]}$ The fluorescence intensity of $\mathrm{Cy} 5$ was measured for $2 \mathrm{~h}$ by sampling the reaction mixture and measuring the aliquots with the plate reader. Three different $\mathrm{O}_{2}{ }^{*-}$ concentrations were monitored $(4,20$ and 40 $\mu \mathrm{M}$ ) and a 3-, 22- and 84-fold increase in fluorescence intensity was observed after $1 \mathrm{~h}$ (Figure 3-5 a). At the highest concentration, the fluorescence intensity increased so dramatically, that after $40 \mathrm{~min}$ the detector was saturated and the sample could not be measured with the same instrument settings (Figure 3-5 b). For the other two concentrations, a non-linear response was observed.

The same cell-free sensing experiments were carried out with a rhodamine-based ROS sensor DHR, a commercially available fluorophore that has been widely employed for measuring the respiratory burst of neutrophils. ${ }^{[196]}$ Even though it was mainly used to detect $\mathrm{O}_{2}{ }^{\bullet-}$, it is not specific for one single ROS but reacts via a radical based mechanism with many reactive species. ${ }^{[46]}$ In the ${ }^{\circ} \mathrm{OH}$ sensing experiments using the Fenton's reagent, a maximal turn-on response of 1.4-fold at the highest concentration was observed (Figure 3-6 a). Similar results in a cell-free set-up were obtained by Henderson et al. who showed that the fluorescence intensity only slowly increased upon $\mathrm{H}_{2} \mathrm{O}_{2}$ addition, but was extremely accelerated when catalase or horseradish peroxidase was added ${ }^{[197]} \mathrm{O}_{2}{ }^{--}$sensing with SOTS-1 proved to be more sensitive for DHR reaching a 8-fold increase. However, a three times higher concentration than tested for HCy5 was required (Figure 3-6 b). 
Overall, oxidation of $\mathrm{HCy} 5$ with $\mathrm{O}_{2}{ }^{-}$and $\mathrm{OH}^{*}$ was possible, and the detected increase in fluorescence intensity was greater than for the well-established DHR. The turn-on response of the synthesized HCy5 was similar to the initial publication of Kundu et al. Considering these promising result of the cell-free assays, the potential of $\mathrm{HCy} 5$ to detect a stress response in cellbased experiments was investigated.

a)

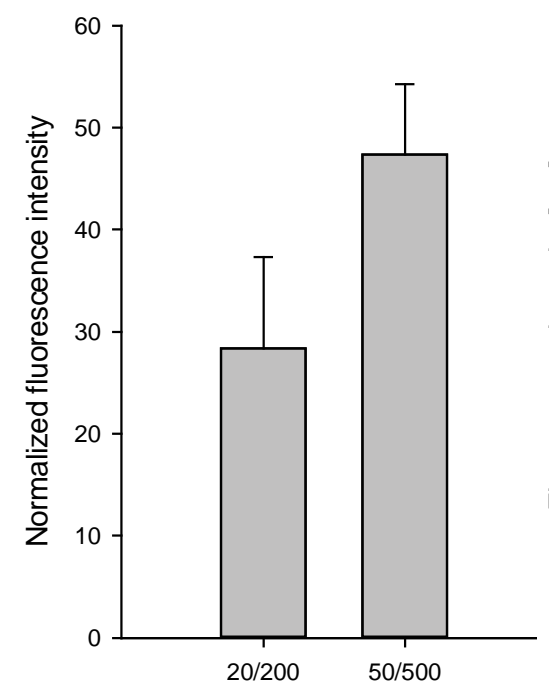

b)

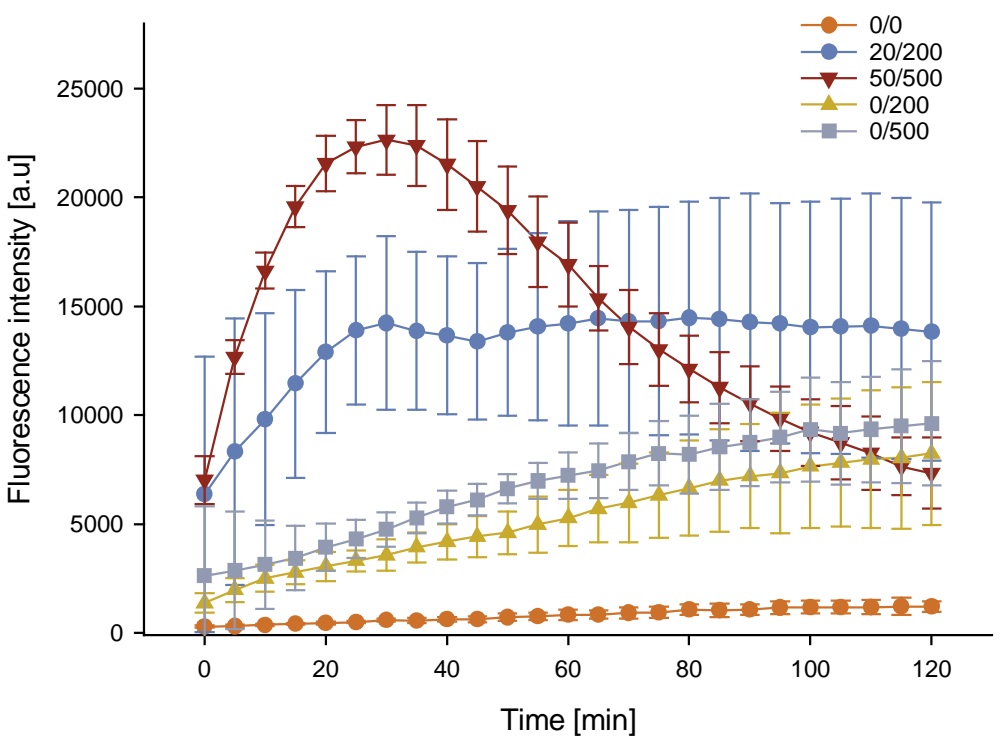

Figure 3-4: Sensitivity of synthesized HCy5 $(10 \mu \mathrm{M})$ towards $\mathrm{OH}^{*}$ generated by the Fenton's reaction $\left(\left[\mathrm{FeSO}_{4}\right] /\left[\mathrm{H}_{2} \mathrm{O}_{2}\right]\right)$ and $\mathrm{H}_{2} \mathrm{O}_{2}$. a) Normalized fluorescence intensity after $20 \mathrm{~min}$. b) Time course of the re-oxidation for $2 \mathrm{~h}$. Legend: $\left[\mathrm{FeSO}_{4}\right] /\left[\mathrm{H}_{2} \mathrm{O}_{2}\right]$ in $\mu \mathrm{M}$. (Mean $\pm \mathrm{SD}, \mathrm{n}=9$ ). 
a)

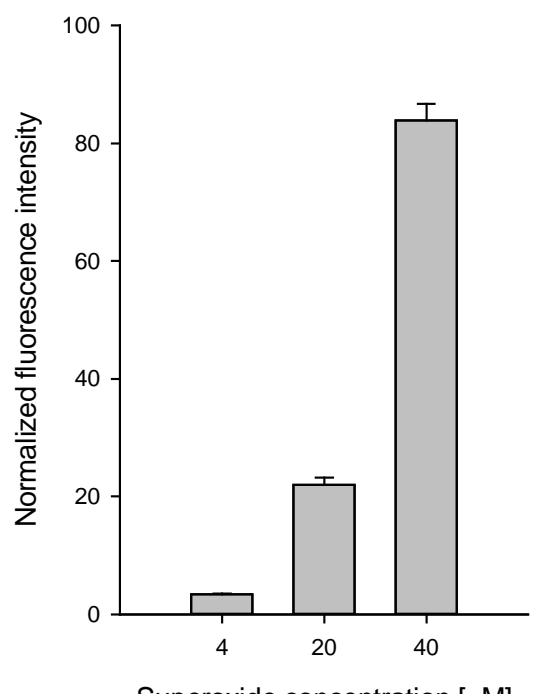

b)

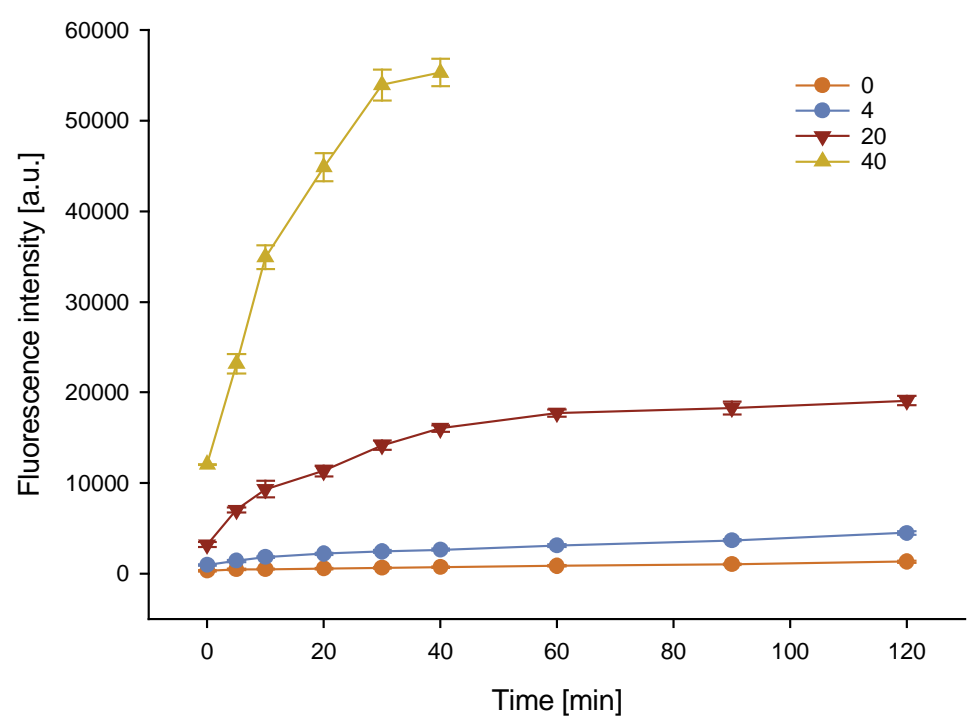

Figure 3-5: Sensitivity of synthesized $\mathrm{HCy} 5$ towards $\mathrm{O}_{2}{ }^{*-}$ generated with SOTS-1, a) Normalized fluorescence intensity after $20 \mathrm{~min}, \mathrm{~b}$ ) Time course of the re-oxidation for $2 \mathrm{~h}$. Legend: $\left[\mathrm{O}_{2}{ }^{\circ}\right]$ in $\mu \mathrm{M}(\mathrm{Mean} \pm \mathrm{SD}, \mathrm{n}=6)$.

a)

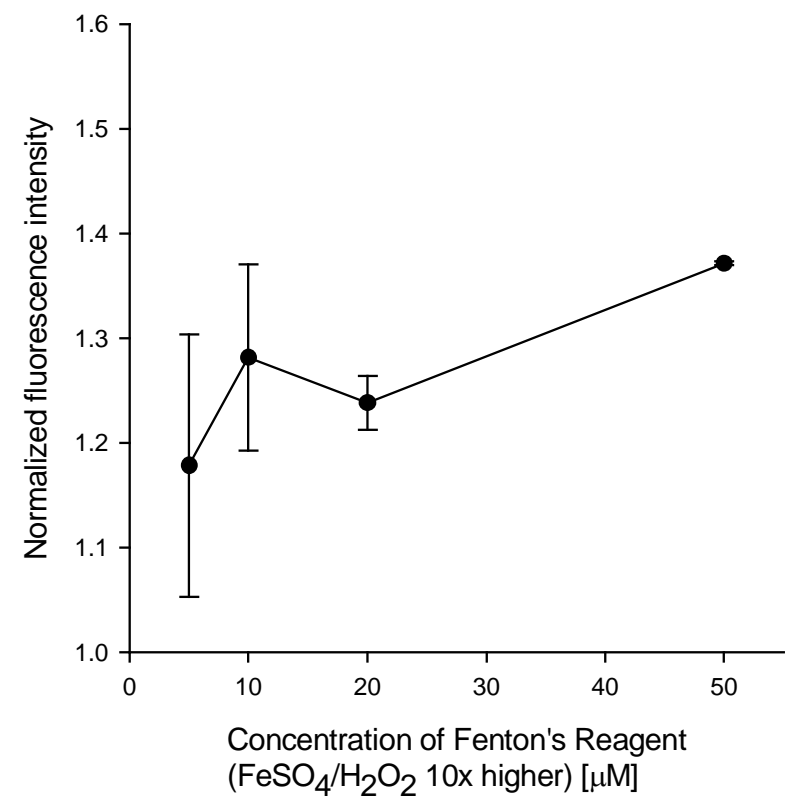

b)

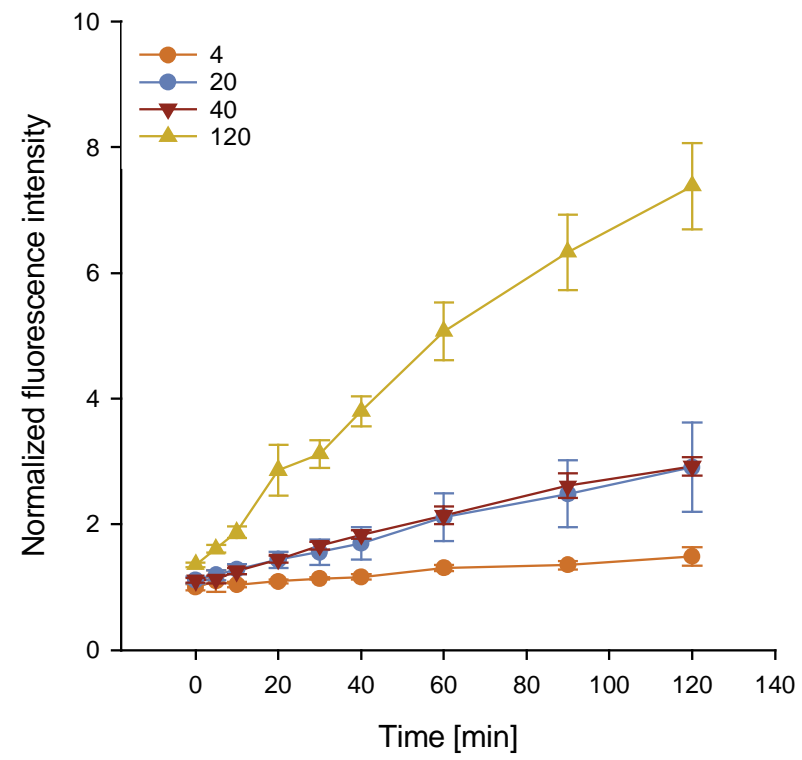

Figure 3-6: Sensitivity of DHR in cell-free assays, a) $\mathrm{OH}^{*}$ sensing using four different concentrations of Fenton's reagent, b) $\mathrm{O}_{2}{ }^{*-}$ radical sensing with SOTS-1 at four different concentrations (Mean $\pm \mathrm{SD}, \mathrm{n}=3$ ). 


\subsubsection{Scratch-wounding experiment with differentiated Caco-2 cells}

During intestinal inflammation, recruitment of neutrophils is one of the first line defense mechanisms of the body as explained in Chapter 1. ROS generation by phagocytic NADPH oxidases is one of the major sources of these transient molecules, but it was shown that this enzyme is also expressed in epithelial cells. ${ }^{[20]} \mathrm{A}$ well-established model to mimic epithelial cells of the intestine are differentiated Caco- 2 cells, which form a polarized monolayer when cultured for two weeks. For this project, Caco-2 cells were the model cell line chosen to not only study stress responses towards various stimuli, but also to assess sensor uptake.

A typical characteristic of Caco-2 differentiation is the formation of tight junctions between single cells, which decreases the permeability of the monolayer. The differentiation process was followed by measuring the increase in resistance of the cell monolayer on a tissue culture insert. To validate our cell model, the unit area resistance was measured during 21 days. After a constant increase of resistance for the first 14 days, a plateau was reached and the cells were considered as ready for the experiments (Figure 3-7 a). The same tissue culture inserts were fixed and embedded in paraffin to examine the morphology of the monolayer by histological cuts $(10 \mu \mathrm{m})$. Pictures were taken after hematoxylin and eosin staining and polarized cells are clearly visible on the support (Figure 3-7 b).

a)

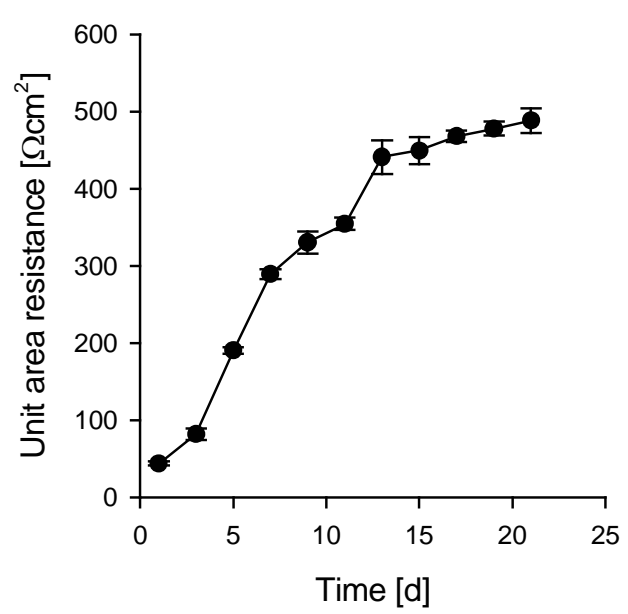

b)

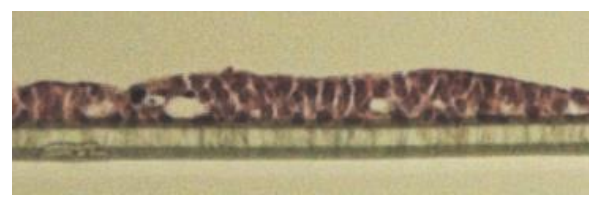

Figure 3-7: Characterization of differentiated Caco-2 monolayer, a) TEER measurement over 3 weeks (Mean \pm SD, $n=3)$, b) Hematoxylin and eosin stained histological cuts $(10 \mu \mathrm{m})$ of differentiated Caco- 2 cells grown on a transwell insert. 
A stress response was first induced by wounding the differentiated Caco-2 monolayer. This physical stress was applied by scratching the cell monolayer with a glass pipette. Subsequently a $20-\mu \mathrm{M}$ HCy5 solution was added, and the fluorescence intensity around the wound was monitored for 65 min with a microscope (Figure 3-8 a-c), and three randomly chosen areas on the scratch border were quantified (Figure 3-8 d). A constant increase of fluorescence intensity was observed during the first $40 \mathrm{~min}$, which reached a plateau after $1 \mathrm{~h}$ at a 2 - to 3-fold fluorescence intensity increase. This experiment suggested that the cells at the border of the injury were affected by the physical wounding and showed a stress response that could be detected with HCy5. Encouraged by these results, an experimental set-up in which all cells were affected and that allowed to use a plate reader was established. Unfortunately, differentiated Caco-2 cells are very insensitive to many stimuli and it was not possible to stimulate a differentiated monolayer. Thus, non-differentiated Caco-2 cells were used for the second stimulation assay.

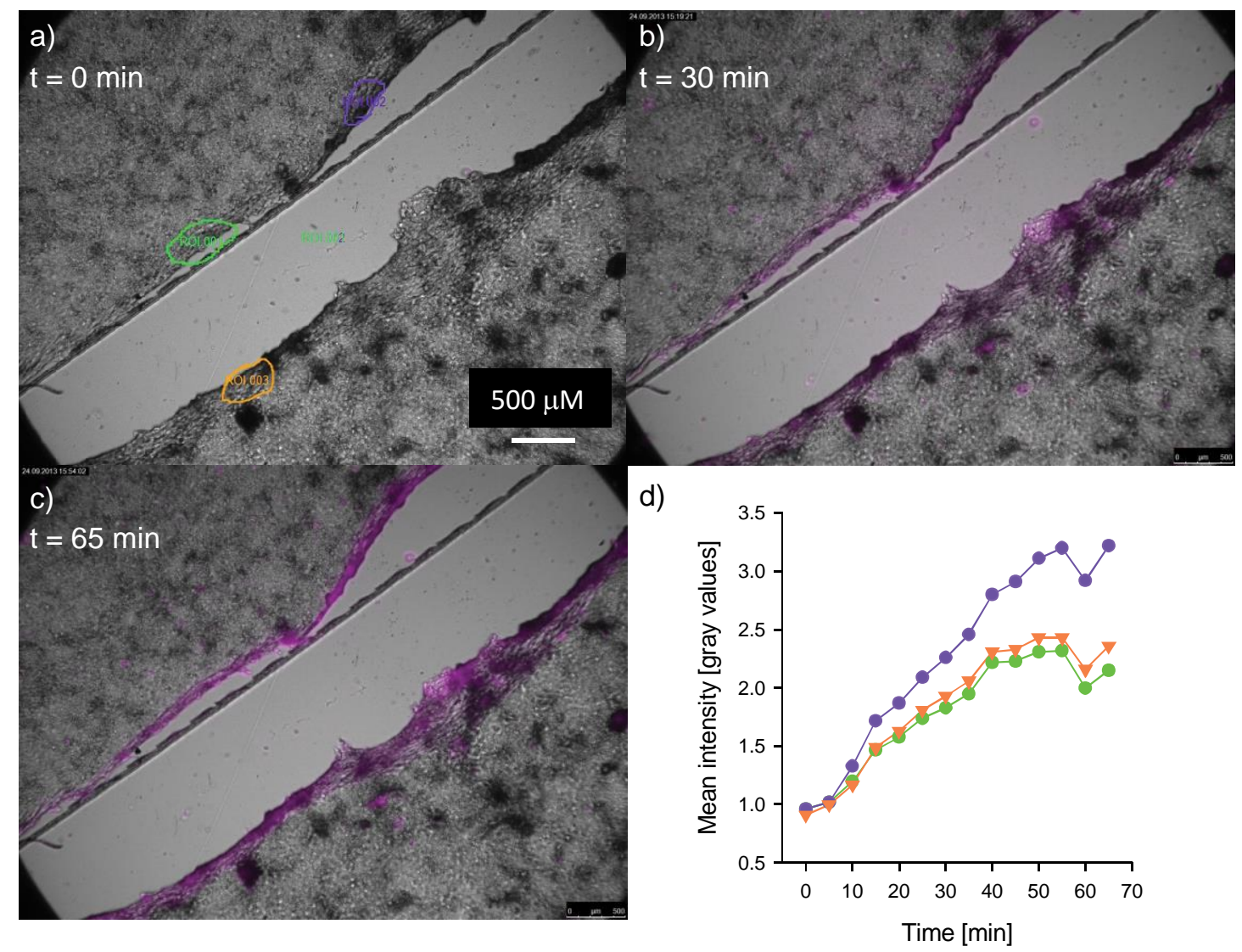

Figure 3-8: Stress response of a differentiated Caco-2 monolayer to a physical injury. The cell monolayer was wounded with a glass pipette and HCy5 $(20 \mu \mathrm{M})$ was monitored for 65 min to follow the stress response, a) $t=0 \mathrm{~min}, \mathrm{~b}) \mathrm{t}=30 \mathrm{~min}, \mathrm{c}) \mathrm{t}=65 \mathrm{~min}, \mathrm{~d}$ ) quantification of colored areas depicted in a). 


\subsubsection{ROS detection in non-differentiated Caco-2 cells}

Addition of stimulants that trigger a stress response is another way to generate ROS in a tissue culture model. After a screening of possible stimulants, $\mathrm{H}_{2} \mathrm{O}_{2}$ and AAPH were used to induce elevated levels of ROS. The performance of HCy5 was compared to the two oxidation-sensitive dyes DCFHDA and DHR, that are regularly used in biological systems to detect ROS. Twentyfour hours after seeding, $\mathrm{H}_{2} \mathrm{O}_{2}$ or AAPH were added, and the dye response was monitored for $2 \mathrm{~h}$ with the plate reader at $37^{\circ} \mathrm{C}$. Both stimulants induced a turn-on response of all three fluorophores. The data was normalized to the fluorescence intensity of non-stimulated cells incubated with the same fluorophore (Equation 3-3):

Norm. fluorescence intensity $=\frac{\text { Fluorescence intensity with stimulated cells }}{\text { Fluorescence intensity with non stimulated cells }}$ Equation 3-3

Addition of AAPH led to a steady rise in fluorescence for all three dyes reaching a 8-, 14- and 12-fold intensity increase for HCy5, DCFHDA and DHR, respectively (Figure 3-9 a). With $\mathrm{H}_{2} \mathrm{O}_{2}$ the maximal increase was observed after 20-30 min and a 6-, 3- and 16-fold enhancement of fluorescence intensity was detected for HCy5, DCFHDA and DHR, respectively (Figure 39 b). Overall, HCy5 performed similar to the commercially available ROS sensors, but bore the advantage of emitting light at a wavelength with less background fluorescence $\left(\lambda_{\text {em,Cy5 }}=665\right.$ $\left.\mathrm{nm}, \lambda_{\mathrm{em}, \mathrm{DCFHDA}, \mathrm{DHR}}=520-530 \mathrm{~nm}\right)$. In contrast to cell-free assays, DHR exhibited a stronger increase than $\mathrm{HCy} 5$ for cells stimulated with any of the two stimulants.

a)

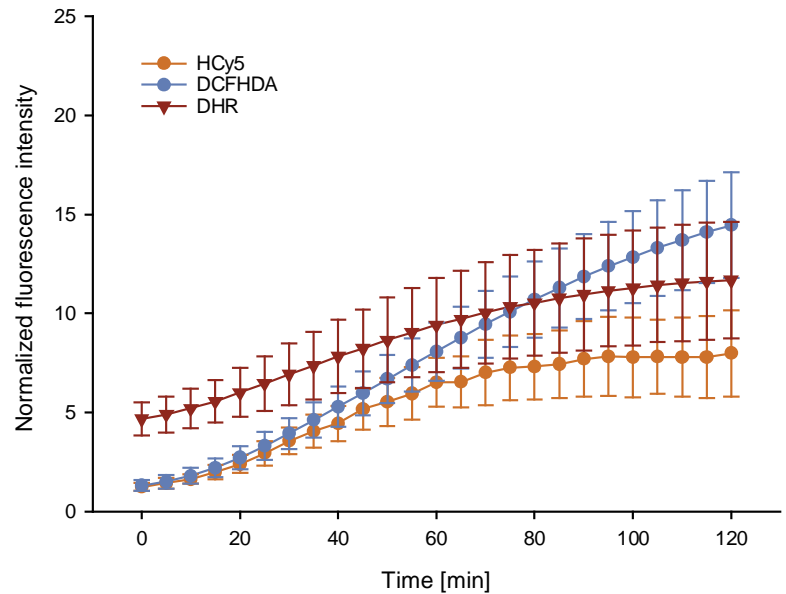

b)

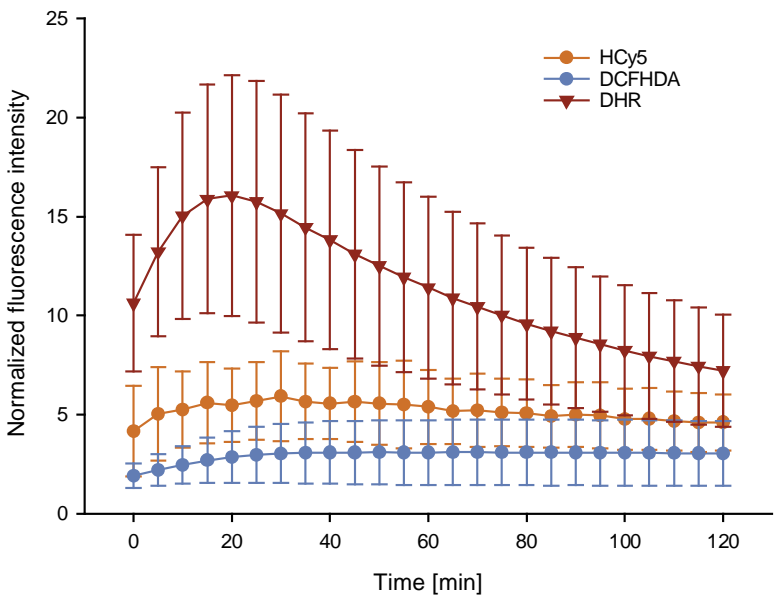

Figure 3-9: Time courses of the normalized fluorescence intensities with non-differentiated Caco- 2 cells with HCy5, DCFHDA and DHR, a) with $1 \mathrm{mM}$ AAPH addition, b) with $1 \mathrm{mM}$ $\mathrm{H}_{2} \mathrm{O}_{2}$ stimulation (Mean $\pm \mathrm{SD}, \mathrm{n}=9-21$ ). 


\subsubsection{Human neutrophils}

To simulate an immune response during intestinal inflammation and to show that also ROS generated from neutrophils can be detected, human neutrophils were isolated from fresh blood samples and stimulated with PMA. Granulocytes and erythrocytes can be separated from other blood components by a density gradient and subsequently erythrocytes are lysed to obtain the granulocyte that are to a large extent neutrophils. After pre-incubation of the fluorophores with neutrophils at $37{ }^{\circ} \mathrm{C}, 200 \mathrm{nM}$ PMA was added to induce the respiratory burst of these immune cells. The fluorescence intensity increase of HCy5, DCFHDA and DHR was monitored for $2 \mathrm{~h}$ with a plate reader at $37{ }^{\circ} \mathrm{C}$. The fluorescence data was normalized as explained for the stimulation assay of non-differentiated Caco- 2 cells. The fluorescence intensity increased steadily for the first 40-60 min and remained constant afterwards (Figure 3-10). DHR exhibited the highest increase (8-fold) and DCFHDA and HCy5 behaved similar for more than an hour reaching a 4-fold increase. In contrast to DCFHDA and DHR, a small decrease of normalized fluorescence intensity was observed for $\mathrm{HCy} 5$ at late time points. Nevertheless, all three dyes can be used to monitor the oxidative burst of human neutrophil in a plate reader set-up and similar sensitivity can be expected.

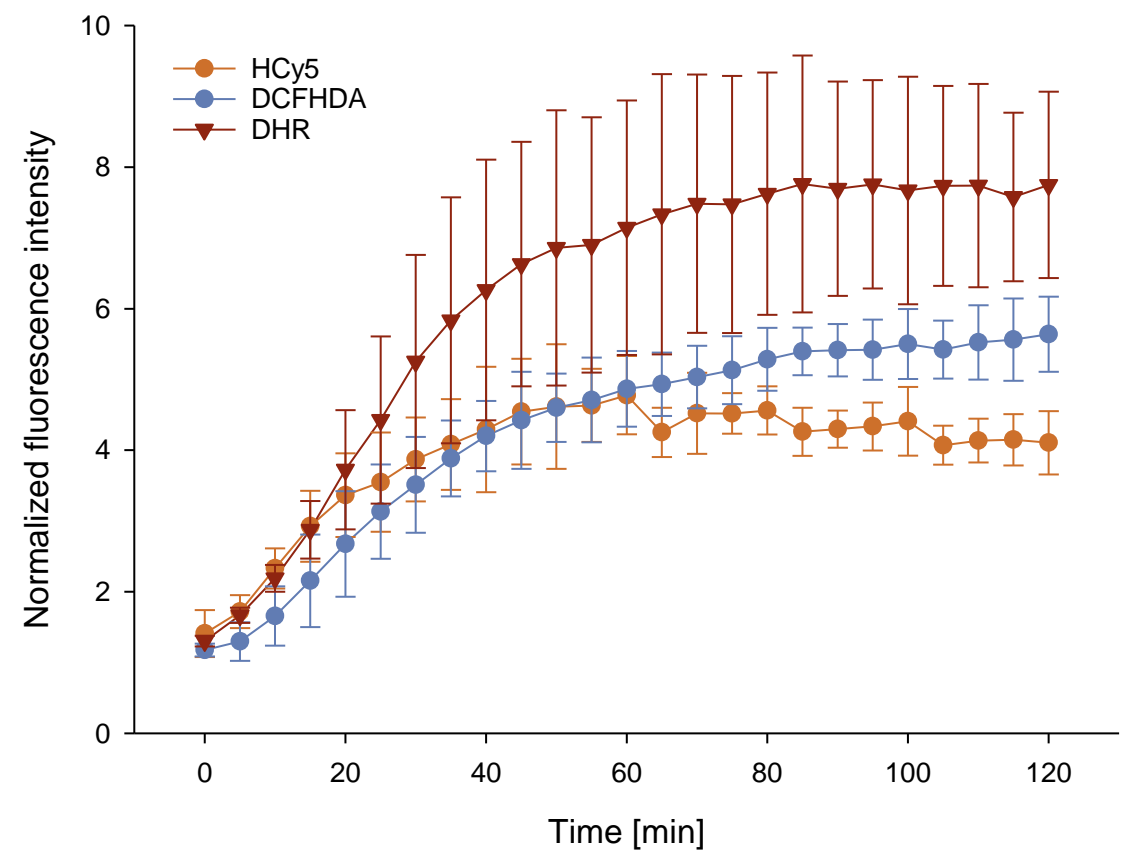

Figure 3-10: Stimulation of human neutrophils with 200 nM PMA. Fluorescence intensity of HCy5, DHR and DCFH was monitored for $2 \mathrm{~h}$ with a plate reader (Mean $\pm \mathrm{SD}, \mathrm{n}=6$ ). 


\subsubsection{Modification of cyanine dyes and uptake in differentiated Caco-2 cells}

Having shown with three cell-based and two cell-free assays that ROS detection with HCy5 was readily achieved, the next step was to modify the sensor to minimize uptake of the probe by cells. PEGylation of small hydrophobic molecules has been a strategy to increase solubility and prolong their biological half-life. ${ }^{[198]}$ Applying the same strategy to HCy5, an amine terminated PEG 20k was reacted with a Cy5-NHS ester. After purification with a size exclusion column, Cy5-PEG was obtained (Figure A7). The effect of the reducing agent $\left(\mathrm{NaBH}_{4}\right)$ on the re-oxidation capacity was investigated, and no influence of the amount of $\mathrm{NaBH}_{4}$ used to reduce Cy5-PEG to HCy5-PEG was observed (Figure 3-12 a). In all cases, the turn-on response was dramatically reduced compared to the $\mathrm{HCy} 5$ and a 2.5 -fold increase in fluorescence intensity was achieved maximally (Figure 3-12 a). A similar reduction of sensitivity was found in $\mathrm{O}_{2}{ }^{*-}$ sensing using SOTS-1 and only a 2.6-fold enhancement of fluorescent intensity was detected (Figure 3-12 b). However, ROS detection was still possible and, therefore, we tested the internalization by differentiated Caco- 2 cells. After $1 \mathrm{~h}$ incubation at $37{ }^{\circ} \mathrm{C}$ of a trypsinized monolayer, significantly less PEG-Cy5 was taken up compared to the uncoupled Cy5 (Figure 3-11), confirming that attaching a hydrophilic polymer to a small organic fluorophore can increase water solubility and decrease internalization by cells. With the aim of designing a ratiometric probe, the broad weight distribution of PEG may impose problems during purification and characterization. Therefore, the PEG 20k was replaced by a well-defined, negatively charged peptide, namely a poly(L-glutamic acid) sequence (PGA) consisting of 9 negatively charged residues. The peptide was synthesized by manual SPPS using a Rink-amide resin. Cy5 was coupled to the $\mathrm{N}$-terminus of the peptide directly on the resin. After obtaining the soluble peptide (Figure A8-9), the uptake of Cy5-PGA was tested to investigate whether a 10 times smaller negatively charged peptide had the same effect on internalization as PEG 20k. The behavior of Cy5-PGA was similar to Cy5-PEG, and the uptake in cells was lower compared to the uncoupled Cy5 (Figure A10). 
a)

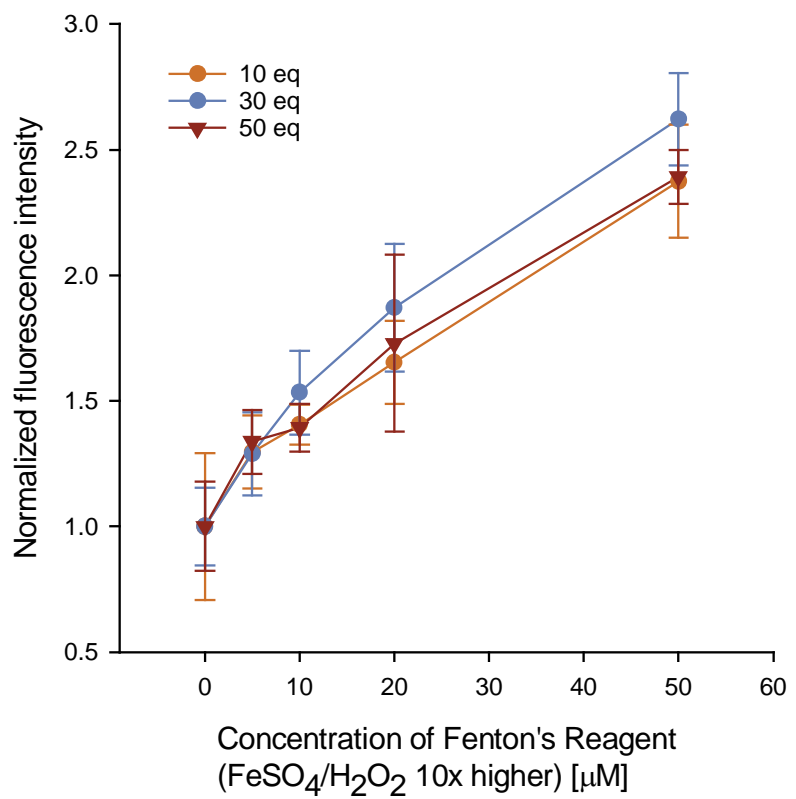

b)

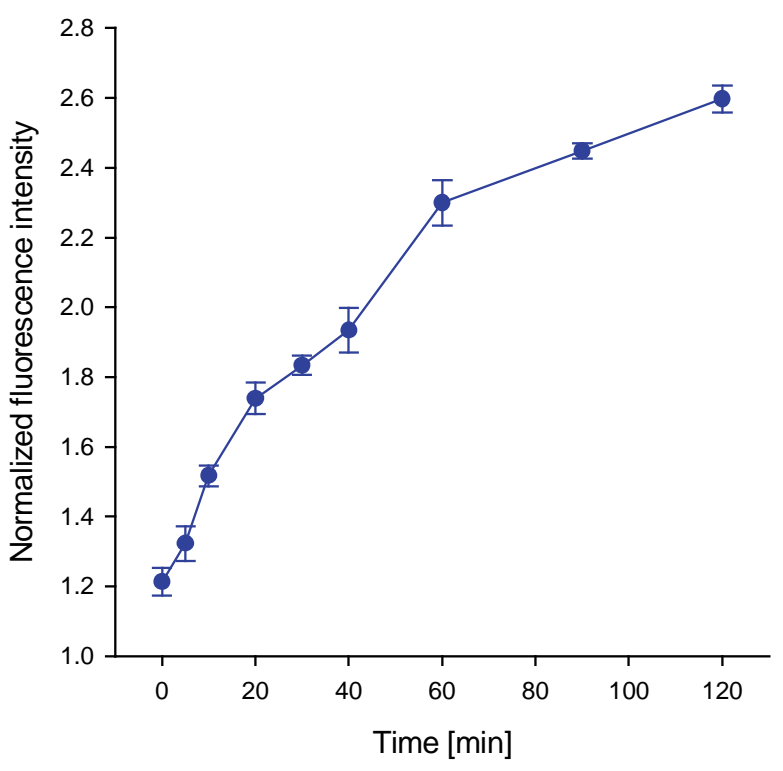

Figure 3-12: Sensitivity of HCy5-PEG in cell-free assays, a) $\mathrm{OH}^{\bullet}$ radical sensing using four different concentrations of Fenton's reagent and increasing excess of reducing agent. Legend: amount of $\mathrm{NaBH}_{4}$ used, b) $\mathrm{O}_{2}{ }^{\bullet-}$ sensing with SOTS-1 generating $20 \mu \mathrm{M}$ of ROS (Mean $\pm \mathrm{SD}$, $\mathrm{n}=3)$.

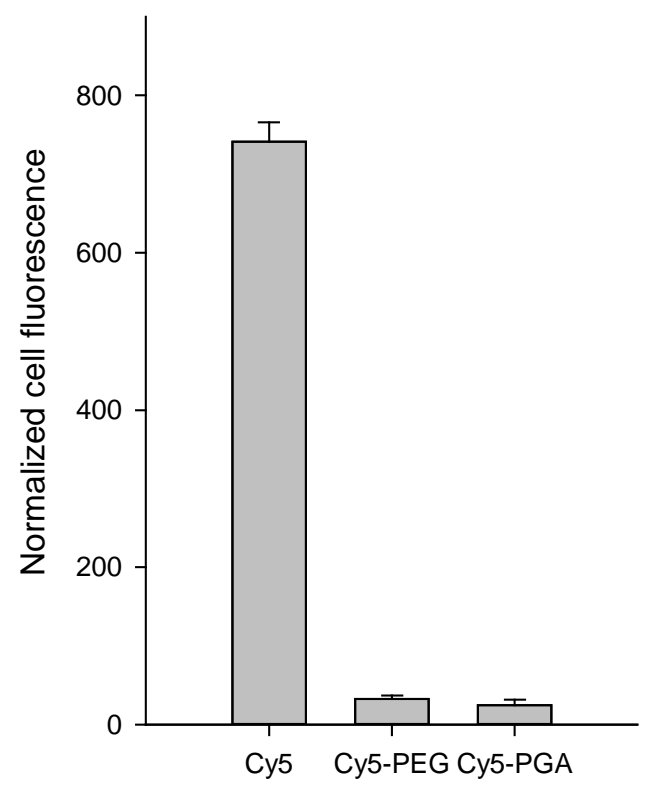

Figure 3-11: Uptake in differentiated Caco-2 cells $($ Mean $+\mathrm{SD}, \mathrm{n}=9)$. 


\subsection{Conclusion}

After an initial screening, $(\mathrm{H}) \mathrm{Cy} 5$ was selected as model sensor for this study. The in vitro behavior of this indicator was first investigated in two cell-free assays by generating $\mathrm{OH}^{*}$ and $\mathrm{O}_{2}{ }^{\circ}$. A strong increase in fluorescence was observed, and was much more prominent than with the control sensor DHR. Three cell-based assays were established to validate the ROS detection capacity of $(\mathrm{H}) \mathrm{Cy} 5$. The border of a scratch wound in a differentiated Caco-2 monolayer lighted up when incubated with the ROS sensor. A stress response was observed with nondifferentiated stimulated Caco-2 cells and stimulated human neutrophils. In both cases the control dyes DCFHDA and DHR showed a comparable behavior as HCy5. To obtain an extracellular probe, Cy5 was first modified with a large PEG to reduce uptake by cells. This was readily achieved, but the ROS sensitivity was greatly reduced compared to the uncoupled (H)Cy5. A better defined, smaller and negatively charged PGA was then synthesized to obtain a platform to build up a ratiometric probe. Cy5-PGA also stayed mainly extracellular and, therefore, represented a valuable starting point for the design of a ratiometric fluorescent probe. 


\section{Chapter 4}

\section{Chromis-PGA-( PEG $\left._{10}\right)_{\mathrm{x}}-(\mathrm{H}) \mathrm{Cy} 5$}

Part of this chapter is published in:

D. Andina, D. Brambilla, N. Munzinger, J. Frei, C. Zivko, J.-C. Leroux, P. Luciani, Development of a Modular Ratiometric Fluorescent Probe for the Detection of Extracellular Superoxide, Chem. - A Eur. J. 2017, 23, 4765-4769. 


\subsection{Introduction}

As the fluorescence intensity of turn-on probes, such as $(\mathrm{H}) \mathrm{Cy} 5$, is inherently dependent on their concentration, undesired signal fluctuation due to concentration differences or sample heterogeneity can occur. An elegant design strategy to circumvent this issue are ratiometric probes, which use a ratio of two fluorescence intensities to obtain a concentration-independent measurement and a change in ratio is usually detected during sensing experiments. Combining a turn-on sensor with a stably emitting fluorophore, that does not react with the analyte of interest (e.g. ROS), is one possibility to create these two fluorescence intensities.

By appending a second dye to the extracellular PGA-(H)Cy5 probe presented in Chapter 3, two fluorescence emission from the same probe - one being ROS sensitive and the other one being ROS-insensitive - could be obtained. To enable incorporation of this reference fluorophore, an additional glutamic acid and a lysine residue were added to the $\mathrm{C}$-terminus of the peptide moiety. The ROS-insensitive indicator was then coupled to the primary amine of the lysine side chain yielding a ratiometric fluorescent probe (Figure 4-1 a).

Considering that the generation of HCy5 required reducing conditions, which may affect the ROS-insensitive dye, the choice of the reporter fluorophore had to be carefully evaluated. Thus, the stability of several fluorophores towards $\mathrm{NaBH}_{4}$ was tested. Chromis500 proved to be the most resistant candidate and was used as reporter dye in the ratiometric fluorescent probes designed in this study.

The well-defined bio-polymeric probe was synthesized by manual SPPS and the number of PEG $_{10}$ spacers was successively increased to minimize the interactions between the fluorophores (Figure 4-1 b). The uptake of the optimized ratiometric probe in differentiated Caco-2 monolayers was assessed and the behavior of the same indicator was studied in aqueous media. Additionally, the reduction with $\mathrm{NaBH}_{4}$ was investigated by monitoring the fluorescence emission of both dyes to ensure that only the Cy5 emission is quenched and the reporter dye remains fluorescent. Finally, validation of the sensing capacity towards $\mathrm{HO}^{\circ}, \mathrm{O}_{2}{ }^{-}$ and $\mathrm{H}_{2} \mathrm{O}_{2}$ was assessed in cell-free assays. The main findings are summarized and discussed at the end of the chapter and possible improvements are proposed. 
a)

Cy5 spacer Chromis

HCy5

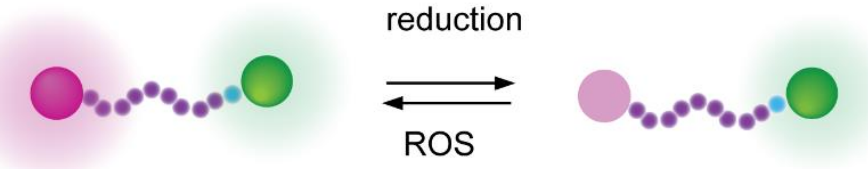

b)

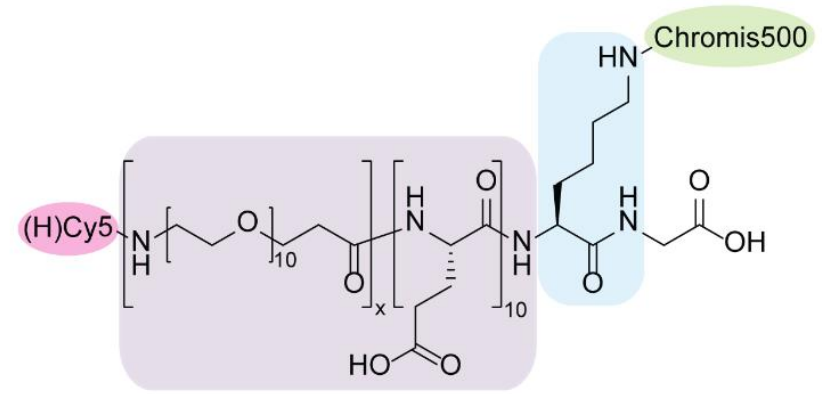

(H)Cy5-(PEG 10$)_{x}-P G A-C h r o m i s$

Figure 4-1: Design of the ratiometric probe Chromis-PGA-( $\left.\mathrm{PEG}_{10}\right)_{\mathrm{x}}-(\mathrm{H}) \mathrm{Cy} 5$, a) Schematic illustration, b) Chemical structure. 


\subsection{Experimental Section}

\subsubsection{Materials}

Additional to the materials listed in Chapter 3 following compounds were used: 2-chlorotritylGly-OH resin and Atto 465 were purchased from Sigma Aldrich (Buchs, Switzerland). FmocLys(Boc)-OH were purchased from Merck Millipore (Billerica, MA). Fmoc-NH-PEG10$\mathrm{CH}_{2} \mathrm{CH}_{2}-\mathrm{OH}$ was obtained from ChemPep Inc. (Wellington, FL). Chromeo ${ }^{\mathrm{TM}} 488$ carboxylic acid was purchased from Active-Motif (La Hulpe, Belgium). 3-bodipy-propanoylaminocaproic acid $N$-hydroxysuccinimide ester (BODIPY) was from Molecular Probes (now Thermo Fisher, Reinach, Switzerland). BDP FL ester was purchased from Lumiprobe (Hannover, Germany). Tris(hydroxymethyl)aminomethane (TRIS) was obtained from BioSolve (Dieuze, France).

\subsubsection{Screening of reporter dyes}

Different amounts $\left(0,5,10,20\right.$ and 50 eq) of $\mathrm{NaBH}_{4}$ in $\mathrm{MeOH}$ were added to a dye solution $(100 \mu \mathrm{M})$ in $\mathrm{MeOH}$. The fluorescence intensity of each dye was measured on a plate reader. BDP: $\lambda_{\mathrm{ex}}=488, \lambda_{\mathrm{em}}=525 \mathrm{~nm}$, Chromis $500 \mathrm{~N}: \lambda_{\mathrm{ex}}=470, \lambda_{\mathrm{em}}=516 \mathrm{~nm}$, Atto 465: $\lambda_{\mathrm{ex}}=430$, $\lambda_{\mathrm{em}}=498 \mathrm{~nm}$, BODIPY: $\lambda_{\mathrm{ex}}=490, \lambda_{\mathrm{em}}=523 \mathrm{~nm}$. The experiment was repeated 3 times in triplicates. The data were normalized to the sample with no $\mathrm{NaBH}_{4}$.

\subsubsection{Synthesis of Chromis-PGA-(PEG 10$)_{x}-\mathrm{Cy} 5$}

\section{Peptide synthesis}

Solid phase peptide synthesis (SPPS) was performed as explained in 3.2.14. Instead of a Rink amide resin, a 2-chlorotrityl-Gly-OH resin was used and one Fmoc-Lys(Boc)-OH and ten Fmoc-Glu $\left({ }^{t} \mathrm{Bu}\right)-\mathrm{OH}$ were coupled to the resin, leading to resin-Gly-Lys(Boc)-Glu $\left({ }^{t} \mathrm{Bu}\right){ }_{10}-\mathrm{Fmoc}$. After Fmoc de-protection, Fmoc-NH-PEG ${ }_{10}-\mathrm{CH}_{2} \mathrm{CH}_{2}-\mathrm{OH}$ (2.5 eq) was activated with HATU (2.4 eq) in DMF for $10 \mathrm{~min}$. Then DIPEA ( $5 \mathrm{eq}$ ) was added and the mixture was transferred to the syringe and shaken for $90 \mathrm{~min}$. The resin was washed 5 times with DMF using 3 resin volumes. This procedure was repeated $\mathrm{x}$ times $(\mathrm{x}=0-6)$.

Cy5-OH coupling and cleavage of the peptide from the resin was carried out as for PGA-Cy5. All compounds were analyzed with HRMS-MALDI and used without further purification. 


\section{Chromis-NHS coupling}

Lys-PGA-(PEG 10$)_{x}-\mathrm{Cy} 5(\mathrm{x}=0-6)$ was dissolved in dry DMF $(200 \mu \mathrm{L})$, then DIPEA (10 eq) and Chromis500-NHS (3 eq) were added. The reaction was stirred for $5 \mathrm{~h}$ at room temperature. $5 \mathrm{~mL} \mathrm{H} \mathrm{H}_{2} \mathrm{O}$ were added and the reaction mixture was lyophilized. The product was dissolved in $\mathrm{H}_{2} \mathrm{O}$ and extracted with $\mathrm{Et}_{2} \mathrm{O}$ until the organic phase was colorless. The crude product was purified by semi-preparative HPLC $\left(\mathrm{XBridge}^{\mathrm{TM}} \mathrm{C}_{18} 5 \mu \mathrm{m} 4.6 \mathrm{~mm} \times 250 \mathrm{~mm}, 1 \mathrm{~mL} / \mathrm{min}\right.$ flow rate, isocratic with water/ACN $(+0.1 \%$ TFA, 80:20 v/v) $5 \mathrm{~min}$ followed by a linear gradient to 20:80 water/ACN $(v / v)$ in $20 \mathrm{~min})$. The purity was verified by analytical HPLC and HRMSMALDI was recorded for all compounds.

\subsubsection{Quantification}

Chromis-PGA-PEG0-6-Cy5 were quantified as stated in 3.2.13.

\subsubsection{Energy transfer measurement}

A fluorescence emission scan of Chromis-PGA-( $\left.\mathrm{PEG}_{10}\right)_{0-6-\mathrm{Cy} 5}$ at a $20 \mu \mathrm{M}$ was measured $\left(\lambda_{\mathrm{ex}}\right.$ $=470)$.

The critical distance $\mathrm{R}_{0}$ in Ångström was calculated by Equation 4-1. ${ }^{[199]}$

$$
R_{0}=0.211\left[\frac{\kappa^{2} \Phi_{D} J(\lambda)}{n^{4}}\right]^{\frac{1}{6}}
$$

Equation 4-1

$\kappa^{2}$ is the orientation factor and was assumed to be $2 / 3$. $\Phi$ is the quantum yield of the donor $\left(\Phi_{\text {Chromis } 500}=1\right) . n$ is the refractive index of the medium $\left(\mathrm{H}_{2} \mathrm{O}=1.33\right)$ and $\mathrm{J}(\lambda)$ is the spectral overlap integral of the fluorescence donor spectrum and the acceptor absorption spectrum (Equation 4-2)

$$
J(\lambda)=\int_{0}^{\infty} \varepsilon_{A}(\lambda) \lambda^{4} F_{D}(\lambda) d \lambda
$$

Equation 4-2

The overlap was calculated using a|e-UV-Vis-IR Spectral Software 2.2 (FluorTools, www.fluortools.com).

The relative FRET efficiency ( $\left.E_{\text {rel }}\right)$ was calculated from the emission scans recorded (Equation 4-3).

$$
E_{\text {rel }}=\frac{I_{A}}{I_{D}+I_{A}}
$$

The approximate length of the spacer (R) was calculated with Equation 4-4. 


$$
R=R_{0} \sqrt[6]{\frac{1-E_{r e l}}{E_{r e l}}}
$$

Equation 4-4

\subsubsection{Dilution of probe}

Cy5-(PEG 10$)_{6}$-PGA-Chromis was diluted to seven concentrations $(1,3,5,10,20,30,50 \mu \mathrm{M})$ in $100 \mathrm{mM}$ TRIS buffer $(\mathrm{pH}=8.5)$. Fluorescence intensity was measured at $\lambda_{\mathrm{ex} / \mathrm{em}}=620 / 665 \mathrm{~nm}$ (Cy5, gain 150) and $\lambda_{\mathrm{ex} / \mathrm{em}}=470 / 516 \mathrm{~nm}$ (Chromis500, gain 80). The ratio of the measured fluorescence intensities was calculated by dividing fluorescence intensity at $665 \mathrm{~nm}$ through intensity at $516 \mathrm{~nm}\left(\mathrm{I}_{\mathrm{Cy} 5} / \mathrm{I}_{\text {Chromis }}\right)$.

\subsubsection{Reduction of Chromis-PGA-(PEG 10$)_{6}-\mathrm{Cy} 5$}

To Chromis-PGA-( $\left.\mathrm{PEG}_{10}\right)_{6}-\mathrm{Cy} 54$ in Tris buffer (pH 8.5) a bubbling $\mathrm{NaBH}_{4}$ solution (1 $\mathrm{mg} / \mathrm{mL}$ ) in $\mathrm{MeOH}$ was slowly added to the blue dye solution. The loss of blue color indicated that the reduction occurs. The fluorescence intensity was measured and the reduction was considered complete if the value obtained was $<1000\left(\lambda_{\mathrm{ex}}=620, \lambda_{\mathrm{em}}=665\right.$, gain 150).

\subsubsection{Re-oxidation with the Fenton's reagent}

$\mathrm{HO}^{*}$ sensing experiments were carried out as described in 3.2.4.

\subsubsection{Re-oxidation with SOTS-1 ${ }^{[193,194]}$}

$\mathrm{O}_{2}{ }^{\bullet-}$ sensing experiments were carried out as described in 3.2.5.

\subsubsection{Uptake in differentiated Caco-2 monolayers}

Cells were seeded into 96-well plates (Nunc) at a density of $5.8 \times 10^{5}$ cells $/ \mathrm{mL}$ and grown for 14-16 days to form a confluent, differentiated monolayer. Medium was exchanged every other day. The growth medium was removed and the monolayer was washed with pre-warmed PBS (100 $\mu \mathrm{L} /$ well). $100 \mu \mathrm{L}$ dye solution $\left(10 \mu \mathrm{M}\right.$ in PBS) was added for $2 \mathrm{~h}$ at $37^{\circ} \mathrm{C}$. The dye solution was removed and the cells were washed with PBS, twice treated with an acid wash (0.2 $\mathrm{M}$ acetic acid and 0.5 M NaCl, $\mathrm{pH} 2.5),{ }^{[195]}$ and washed with PBS. To prepare the cells for flow cytometry, they were detached using $100 \mu \mathrm{L}$ trypsin (0.25\% Trypsin-EDTA) for 15 min. 
The cells were collected in medium and centrifuged $\left(300 \times g, 4{ }^{\circ} \mathrm{C}, 5 \mathrm{~min}\right)$. The supernatant was discarded and each cell pellet was washed once. Flow cytometry measurements (FACSCanto $^{\mathrm{TM}}$ Flow Cytometer) were performed in PBS supplemented with $0.5 \% \mathrm{w} / \mathrm{v}$ BSA and 2 mM EDTA. A minimum of 10,000 events per sample were recorded. The 488-nm laser was used for the Chromis excitation and the FITC channel to detect the fluorescence emission. The 633-nm laser was used for the Cy5 excitation and the APC channel to detect the fluorescence emission. All experiments were carried out in triplicates and two independent experiments.

\subsubsection{Statistical Analysis and Coefficient of variation}

All data are presented as Mean \pm SD and the coefficient of variation (CV) is SD divided by the Mean. The one-way ANOVA (parametric or non-parametric depending on the distribution of the sample groups) test combined with a Dunnett's test was applied for comparison of different groups to a control group. The significant difference was assigned at a p-value of $<0.05$. $*^{*}: \mathrm{p}$ $\leq 0.05, * *: \mathrm{p} \leq 0.01, * * *: \mathrm{p} \leq 0.001)$. 


\subsection{Results and Discussion}

\subsubsection{Screening of ROS-insensitive dye}

The use of HCy5 as ROS-sensor offered the major advantage of introducing the ROS-sensing ability by a reduction step shortly before the sensing experiments, which simplified the synthesis of the probe. However, this also implied that that the reference dye should not be affected by the reducing conditions. In a preliminary screening, several possible reporter fluorophores were exposed to $\mathrm{NaBH}_{4}$ and their stability was assessed with a plate reader by measuring the residual fluorescence intensity. The data were normalized to the initial fluorescence intensity value obtained of the same dye when no $\mathrm{NaBH}_{4}$ was added. In contrast to all other tested dyes (BODIPY F1, BDP Fl and Atto465, Figure 4-2 a) that completely lost their fluorescence properties, Chromis500 retained more than $90 \%$ of its initial fluorescence intensity upon addition of 50 equivalents $\mathrm{NaBH}_{4}$ (Figure 4-2 b). Chromis500 (Chromis) is neutral derivative of the BODIPY family, which are known to be highly fluorescent and exhibit sharp absorption and emission peaks. They were shown to be insensitive to solvent polarity and stable towards variations in $\mathrm{pH}^{[200]}$ These favorable characteristics and the fact that its derivatives, that span the visible spectrum, are readily available, led to the routine use of these fluorophores in bio-imaging. Based on our own findings and its frequent use in literature, Chromis was selected as reporter dye for this study.

\subsubsection{Synthesis, optimization of spacer length and characterization}

The introduction of Chromis into PGA-Cy5, presented in section 3.3.7, required a few modifications of the chemical structure and synthesis conditions. The spacer was assembled with manual standard SPPS (Figure 4-2 a) containing one lysine and ten glutamic acid residues (poly(glutamic acid), PGA). Cy5-OH was coupled to the N-terminus of the last glutamic acid directly on the resin as stated in Chapter 3. However, a 2-chlorotrityl-Gly-OH resin instead of a Rink amide resin was used to obtain higher yields when the peptide was cleaved from the resin. The primary amine of the introduced lysine amino acid was later used to condensate activated Chromis to the spacer-Cy5 derivative (Figure 4-2 b) obtaining Chromis-PGA$\left(\mathrm{PEG}_{10}\right)_{0}-\mathrm{Cy} 5$. 
a)<smiles>Cc1cc(C)[n+]2p(F)n3c(CCC(=O)ON4C(=O)CCC4=O)ccc3cc1-2</smiles>

BDP FL Bodipy FL<smiles></smiles><smiles>Cc1cc(C)[n+]2c(CCCCCn3cc(CCCC(=O)ON4C(=O)CCC4=O)nn3)c1-c1c(C)cc(C)n1B2F</smiles>

b)

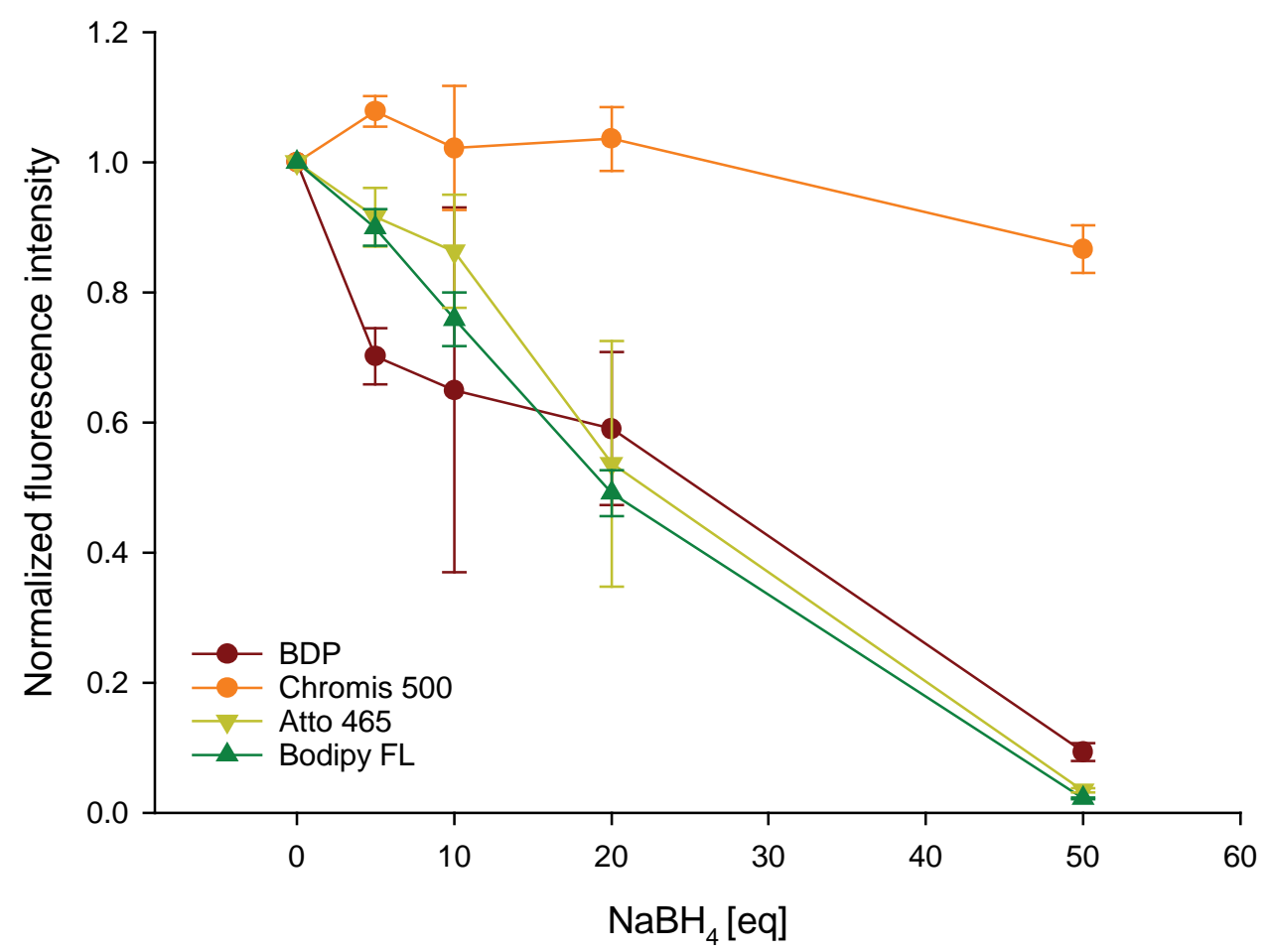

Figure 4-2: a) chemical structure of BDP, Bodipy FL, Atto 465 and Chromis500-NHS, b) effect of $\mathrm{NaBH}_{4}$ on different reporter dye candidates. $(n=3$, Mean $\pm \mathrm{SD})$. 
a)

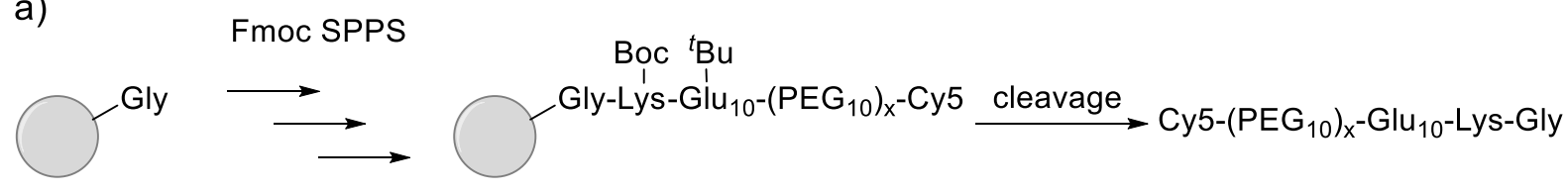

b)

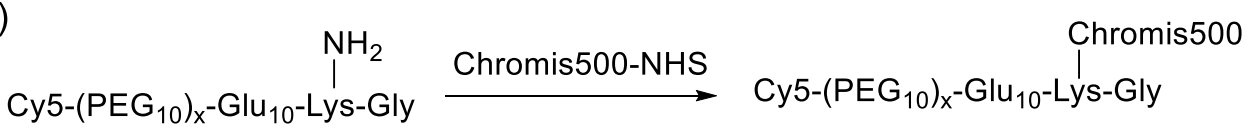

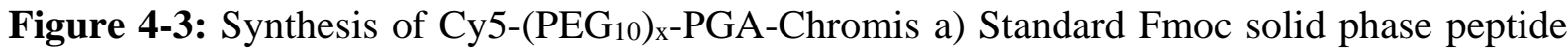
synthesis, b) Coupling of Chromis500 to the peptide.

During spectrophotometric characterization, two fluorescence emission peaks were observed upon excitation of Chromis at $470 \mathrm{~nm}$. The lower wavelength emission at $510 \mathrm{~nm}$ was consistent with the usually obtained spectrum of Chromis and the second emission peak at $670 \mathrm{~nm}$ corresponded to Cy5 fluorescence (Figure 4-4, green line). The appearance of a Cy5 emission peak at $670 \mathrm{~nm}$, indeed without exciting the cyanine dye, was attributed to a fluorescence resonance energy transfer (FRET) from Chromis to Cy5. ${ }^{[201]}$ A FRET effect was undesired for this system as it only occurs when the ROS sensor is switched-on, considerably complicating the final signal read-out. Taking into account that FRET efficiency is inversely proportional to the sixth power of the distance from donor to acceptor, ${ }^{[201]}$ the inter-fluorophore distance was increased subsequently. A library of probes with one to six discrete PEG 10 units

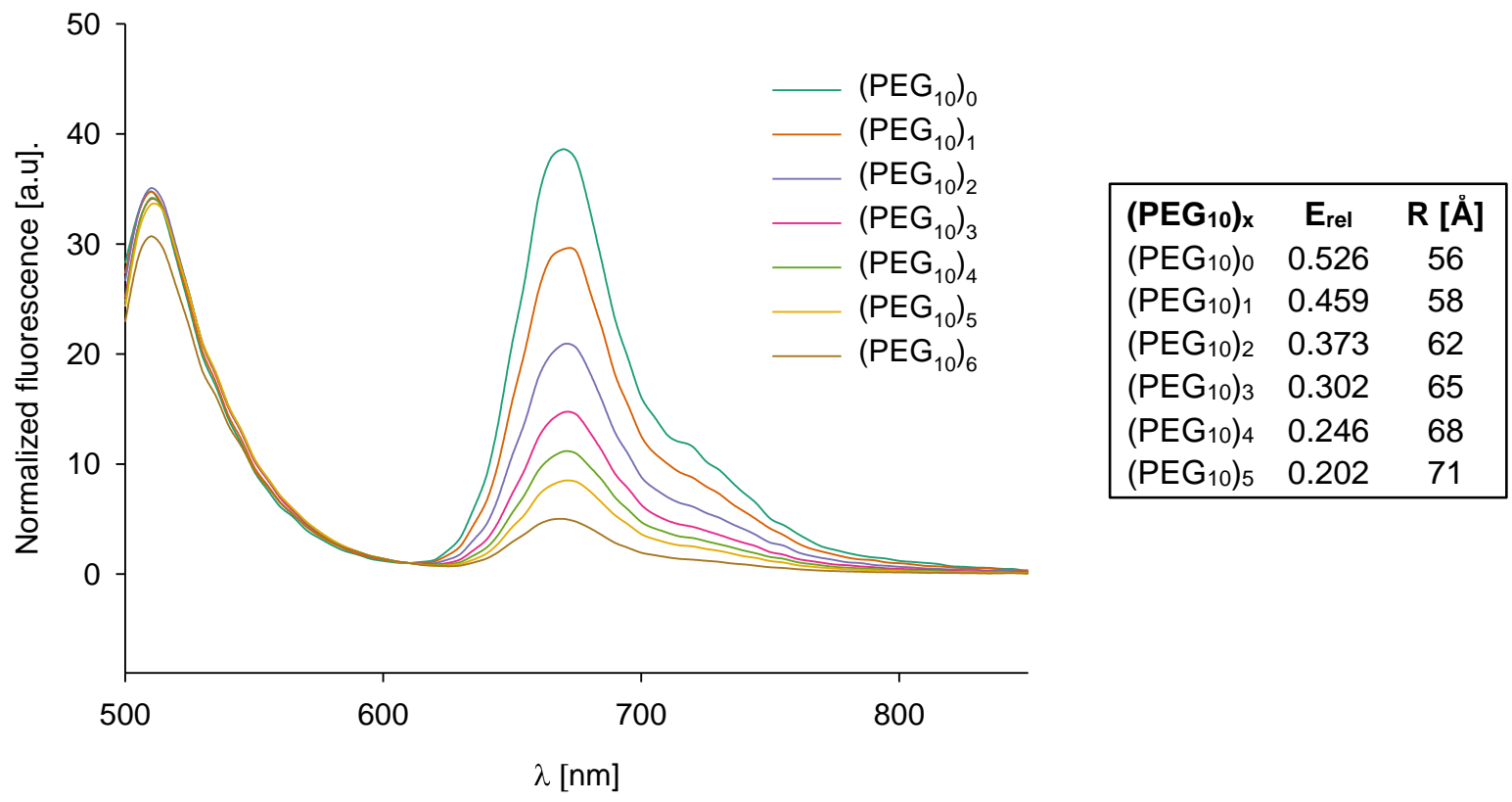

Figure 4-4: Effect of spacer length on FRET efficiency in 4, $\lambda_{\mathrm{ex}}=470 \mathrm{~nm}$, inset: calculation of the approximate length of the spacer unit. Increasing the number of $\mathrm{PEG}_{10}$ spacers resulted in a reduction of $E_{\text {rel }}$ and an increased distance between the two dyes. 
was prepared to compare the effect of spacer length. The PEG10 units were inserted after the PGA and synthesis was carried out as stated for Chromis-PGA-(PEG 10$)_{0}-\mathrm{Cy} 5$ (Figure 4-3). All ratiometric probes were purified by semi-preparative HPLC and the obtained compounds were analyzed by HPLC and MALDI mass analysis (Figure A11-31). As anticipated, a pronounced reduction of FRET was observed with increasing number of $\mathrm{PEG}_{10}$ units (Figure 4-4). By calculating the relative energy that was transferred and considering the spectral overlap of the fluorophores, an approximate distance between the dyes was calculated (Figure 4-4 box). Incorporating six PEG10 units increased the distance from $56 \AA$ to over $70 \AA$ and resulted in a negligible FRET effect. Thus, Chromis-PGA-(PEG10)6-Cy5 4 was used in all further experiments. Absorption and fluorescence emission spectra did no change compared to the uncoupled fluorophores, exhibiting a sharp absorption and fluorescence emission peaks around $500 \mathrm{~nm}$ and $650 \mathrm{~nm}$ (Figure 4-5).

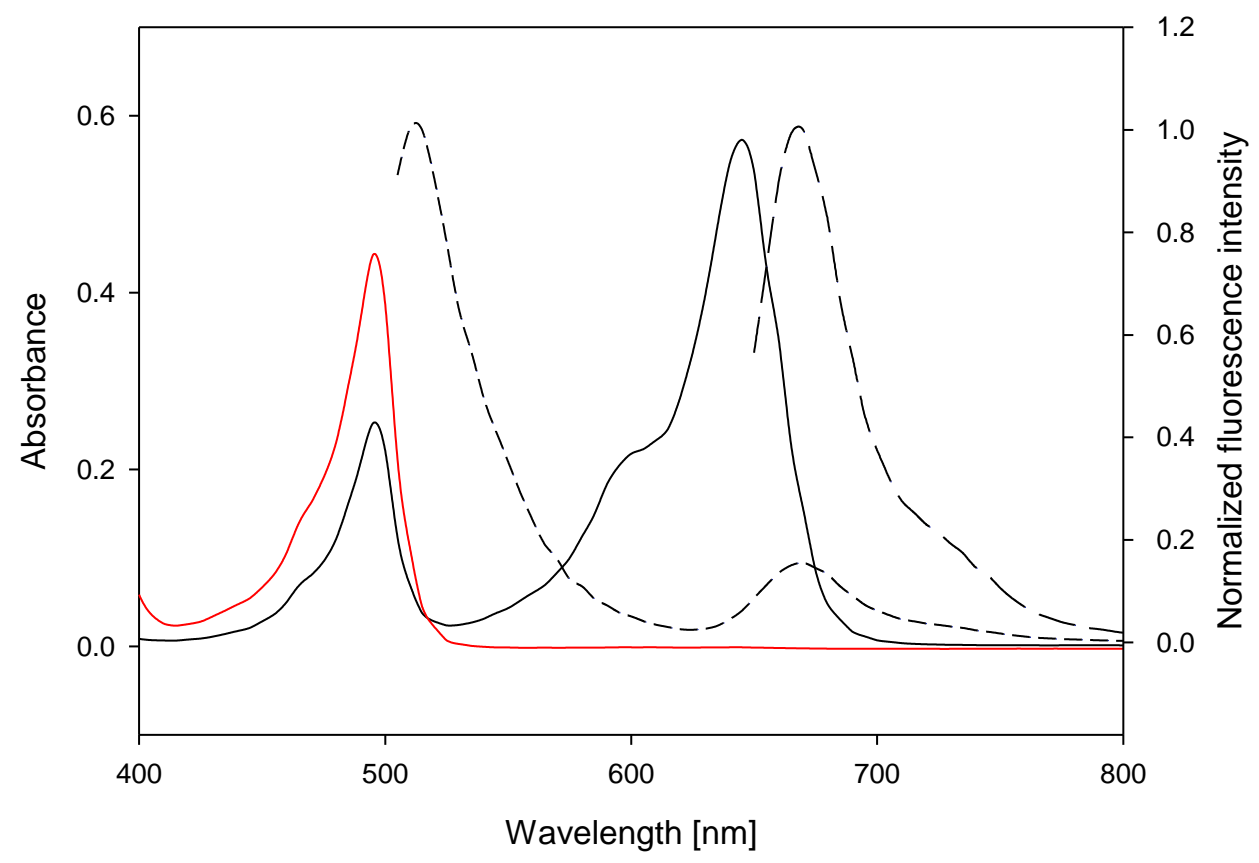

Figure 4-5: Absorbance scan of Chromis-PGA-( $\left.\mathrm{PEG}_{10}\right)_{6}-\mathrm{Cy} 54$ (black line) and ChromisPGA-(PEG 10$)_{6}-\mathrm{HCy} 55$ (red line) and fluorescence emission scans of 4 (dashed black lines). Chromis $\lambda_{\mathrm{ex}}=470 \mathrm{~nm}, \mathrm{Cy} 5 \lambda_{\mathrm{ex}}=620 \mathrm{~nm}$. 


\subsubsection{Chromis-PGA-(PEG 10$)_{6}-\mathrm{Cy} 5$ in Tris-buffered medium}

To study the behavior of Chromis-PGA-( $\left.\mathrm{PEG}_{10}\right)_{6}-\mathrm{Cy} 5$ in Tris-buffer, the fluorescence emissions of $\mathrm{Cy} 5$ and Chromis at different concentrations of the probe were measured and the fluorescence emission ratio $\mathrm{I}_{\mathrm{Cy} 5} / \mathrm{I}_{\text {Chromis }}$ was calculated. The ratio stayed constant at concentrations $<10 \mu \mathrm{M}$, but decreased for concentration $>10 \mu \mathrm{M}$ (Figure 4-6). A decrease in fluorescence emission ratio implied that the emission intensity of Cy5 did not increase to the same extent as the one of Chromis, when the concentration of the probe was increased, indicating possible aggregation and concomitant quenching of Cy5. Therefore, the highest concentration of Chromis-PGA-( $\left.\mathrm{PEG}_{10}\right)_{6}-(\mathrm{H}) \mathrm{Cy} 5$ used in all further experiments was $10 \mu \mathrm{M}$.

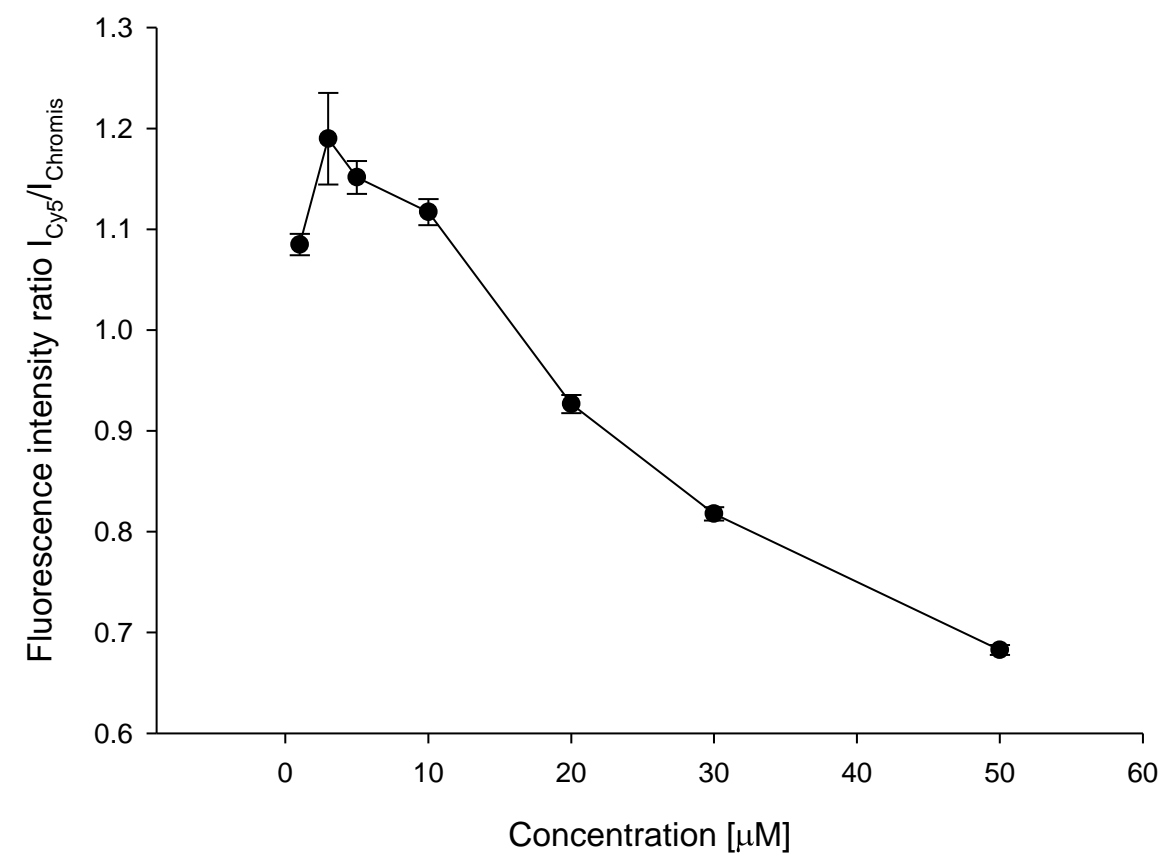

Figure 4-6: Fluorescence intensity ratio $\mathrm{I}_{\mathrm{Cy} 5} / \mathrm{I}_{\text {Chromis }}$ measured in Tris-buffer $(\mathrm{pH}=8.4)$ at seven different concentrations $(1,3,5,10,20,30$ and $50 \mu \mathrm{M}, \mathrm{n}=9$, Mean $\pm \mathrm{SD})$.

\subsubsection{Uptake of Chromis-PGA-(PEG $\left.{ }_{10}\right)_{6}-\mathrm{Cy} 5$ in differentiated Caco-2 monolayers}

Having synthesized a well-defined dual-emissive probe with a minimal FRET effect, uptake studies of Chromis-PGA-( $\left.\mathrm{PEG}_{10}\right)_{6}-\mathrm{Cy} 5$ in differentiated Caco-2 cells were effectuated. After $1 \mathrm{~h}$ incubation of the free dyes and 4 at $3 \mu \mathrm{M}$, the cell monolayer was trypsinized and analyzed by flow cytometry (Figure 4-7). A considerably reduced uptake of $\mathbf{4}$ in comparison with the uncoupled dyes Chromis and Cy5 was observed, confirming that the probe remains largely extracellular. In a last synthetic step, the ROS-sensing ability was introduced by reducing $\mathrm{Cy} 5$ to $\mathrm{HCy} 5$. 
a)

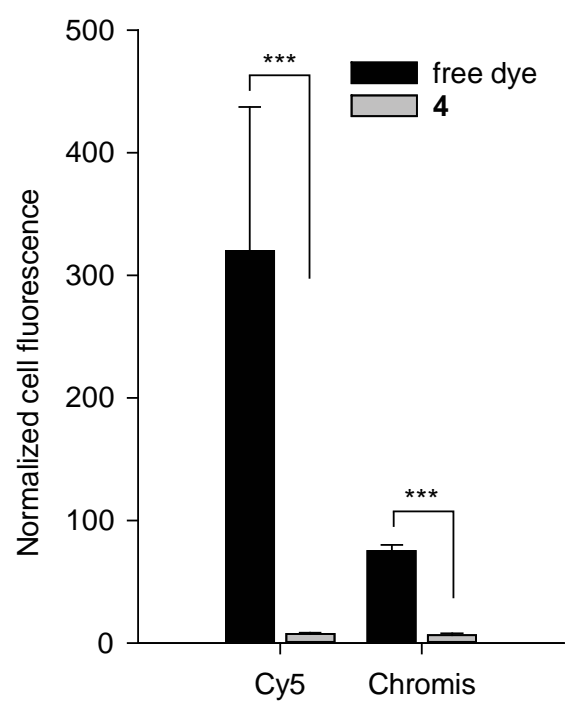

b)

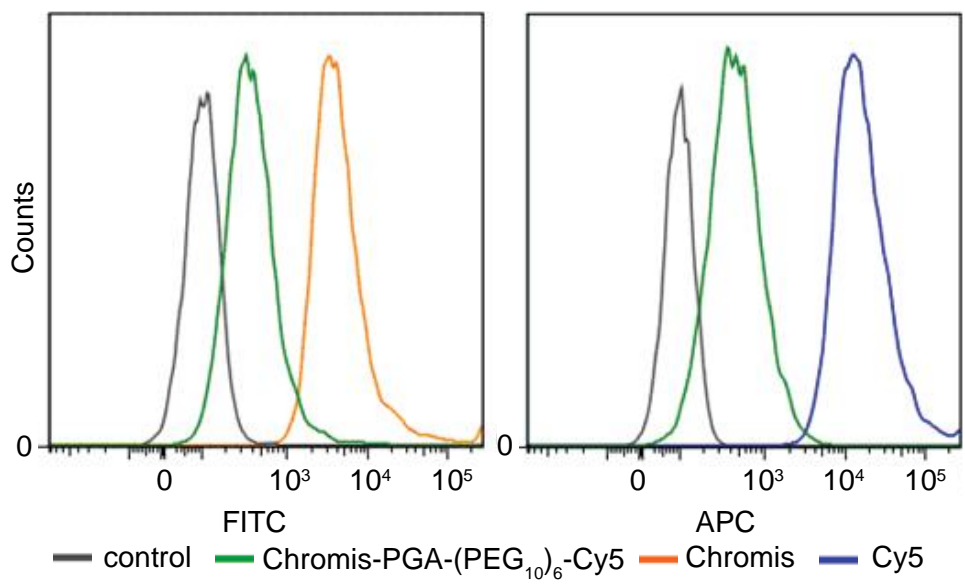

Figure 4-7: Uptake of Chromis-PGA-(PEG10)6-Cy5 4 Cy5 and Chromis in differentiated Caco2 cells at $3 \mu \mathrm{M}$ after $1 \mathrm{~h}$ at $37^{\circ} \mathrm{C}$, a) bar plot of the fluorescence intensity normalized to the intensity of the blank (Mean $+\mathrm{SD}, \mathrm{n}=9$ ), b) representative flow cytometry diagrams, left: filter for Cy5, right: filter for Chromis500.

\subsubsection{Reduction of Chromis-PGA-(PEG $\left.{ }_{10}\right)_{6}-\mathrm{Cy} 54$}

The effect of the reducing agent on the ratiometric molecule was investigated by adding different equivalents of $\mathrm{NaBH}_{4}$ to a solution of 4 . The fluorescence intensity of Chromis almost doubled, then plateaued and stabilized at higher intensity also during all further experiments (Figure 4-8). On the contrary, the Cy5 emission was rapidly suppressed, which confirmed the successful conversion into the ROS-sensor 5 without losing the spectral properties of the reporter dye. The remaining FRET effect present in Chromis-PGA-(PEG10)6-Cy5 4 is disabled upon reduction of $\mathrm{Cy} 5$ to $\mathrm{HCy} 5$ and may partly account for the increase in Chromis intensity observed during $\mathrm{NaBH}_{4}$ addition. 
a)

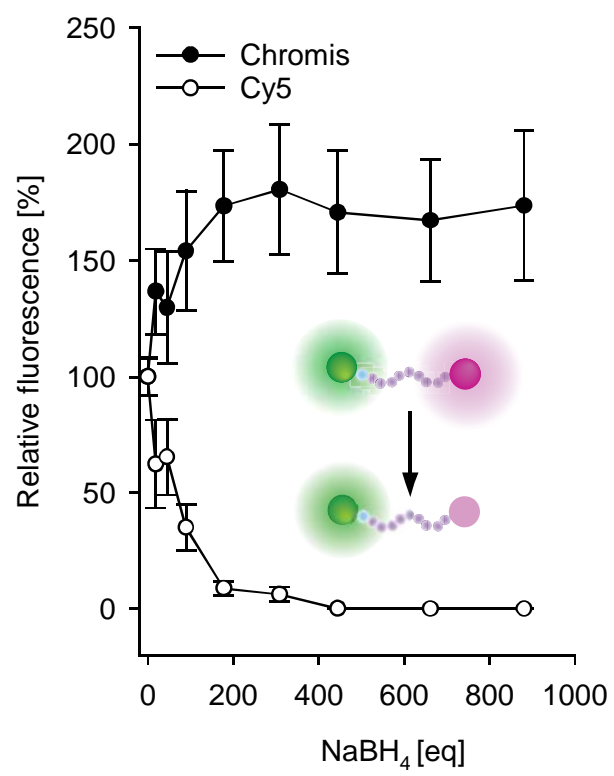

b)

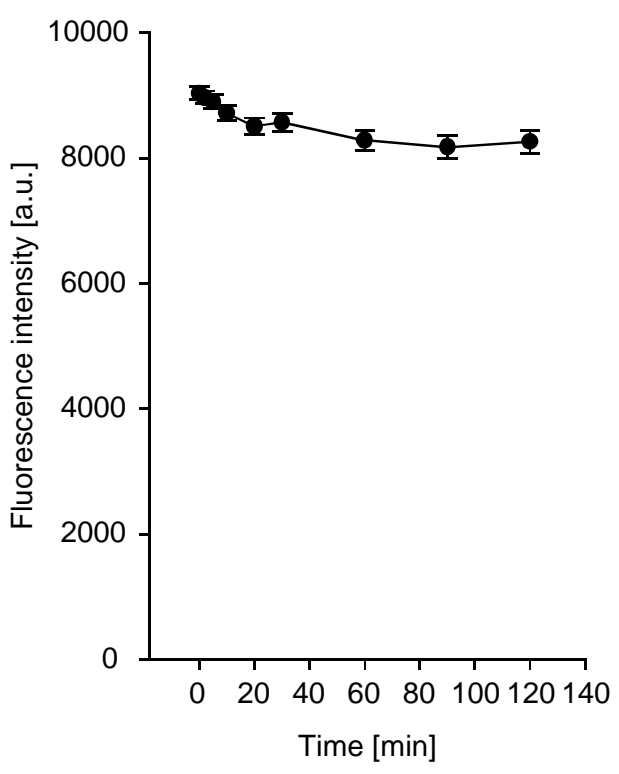

Figure 4-8: a) effect of $\mathrm{NaBH}_{4}$ on Chromis and Cy5 (10 $\mu \mathrm{M}$, Mean $\left.\left.\pm \mathrm{SD}, \mathrm{n}=9\right), \mathrm{b}\right)$ emission of Chromis over $2 \mathrm{~h}$ in Chromis-PGA-(PEG10)6-HCy5 5.

\subsubsection{Sensing of $\mathrm{HO}^{\circ}$ and $\mathrm{O}_{2}{ }^{\circ-}$ radicals and specificity of 5}

The ROS-sensing properties of $\mathbf{5}$ were validated using the same cell-free assays as established for $(\mathrm{H}) \mathrm{Cy} 5$ in Chapter 3. First $\mathrm{HO}^{\circ}$ was generated using the Fenton's reagent and the increase in fluorescence intensity ratio ICy5/IChromis was monitored at $10 \mu \mathrm{M}$. In contrast to the free (H)Cy5, a minor increase in (H)Cy5 signal and a negligible increase in ratiometric signal was observed during the experiment (1.1- to 1.3-fold, Figure 4-9). Two different concentrations (50 and $100 \mu \mathrm{M}$ ) of $\mathrm{O}_{2}{ }^{*-}$ were generated to investigate the sensing capacity of $\mathbf{5}$. After $1 \mathrm{~h}$ a 1.9 and 2.3-fold increase of the fluorescence intensity ratio (Icy5/IChromis) was observed (Figure 4-10) and the ratio at a physiologically relevant concentration ${ }^{[202]}(50 \mu \mathrm{M})$ showed a low coefficient of variation $(\mathrm{CV}, 5 \%)$. However, the conjugation of $\mathrm{HCy} 5$ with the polymer reduced its sensitivity towards the detection of both ROS significantly compared to free $(\mathrm{H}) \mathrm{Cy} 5$. Upon addition of $\mathrm{H}_{2} \mathrm{O}_{2}$ at $200 \mu \mathrm{M}$, no increase in fluorescence intensity ratio was observed, resulting in a specific detection of $\mathrm{O}_{2}{ }^{-}$over $\mathrm{HO}^{\circ}$ and $\mathrm{H}_{2} \mathrm{O}_{2}$ (Figure 4-11). These findings show that the optimized setup of the covalently linked ratiometric ROS-sensing probe Chromis-PGA( $\left.\mathrm{PEG}_{10}\right)_{6}-\mathrm{HCy} 5$ allows specific superoxide detection. 
a)

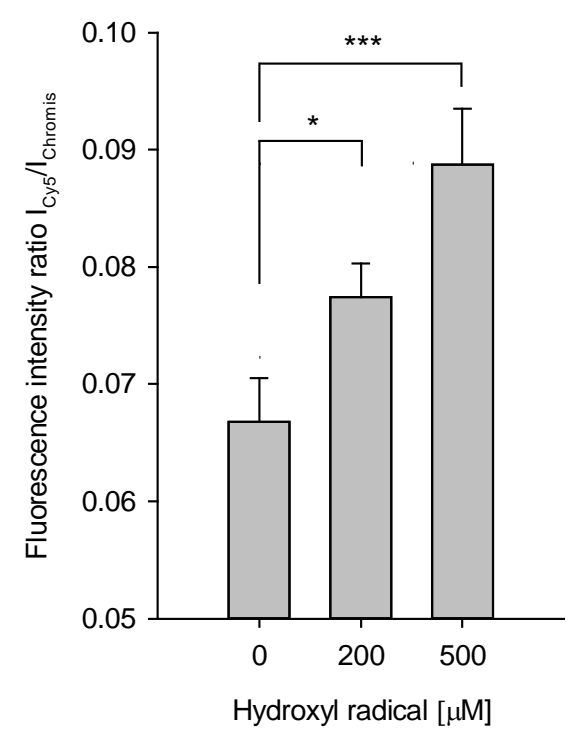

b)

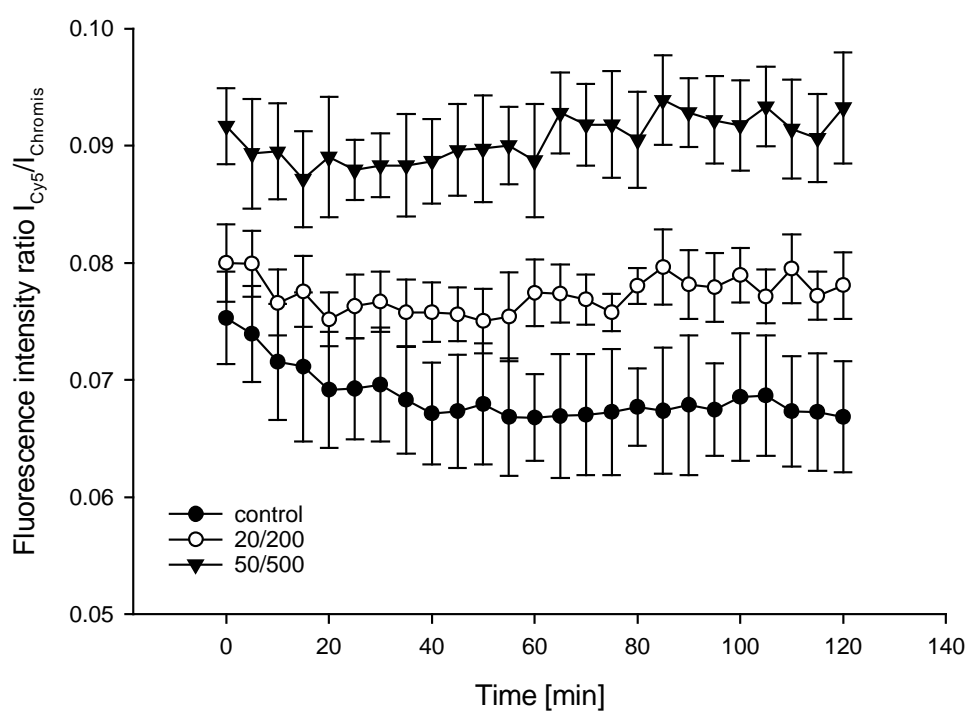

Figure 4-9: Sensing hydroxyl radicals of Chromis-PGA-( $\left.\mathrm{PEG}_{10}\right)_{6}-\mathrm{HCy} 55(10 \mu \mathrm{M})$ with the Fenton's reaction $\left(\left[\mathrm{FeSO}_{4}\right] /\left[\mathrm{H}_{2} \mathrm{O}_{2}\right]\right)$. Legend: $\left[\mathrm{FeSO}_{4}\right] /\left[\mathrm{H}_{2} \mathrm{O}_{2}\right]$ in $\mu \mathrm{M}$. control: 2 at $10 \mu \mathrm{M}$. Mean $\pm \operatorname{SD}(n=9)$.

a)

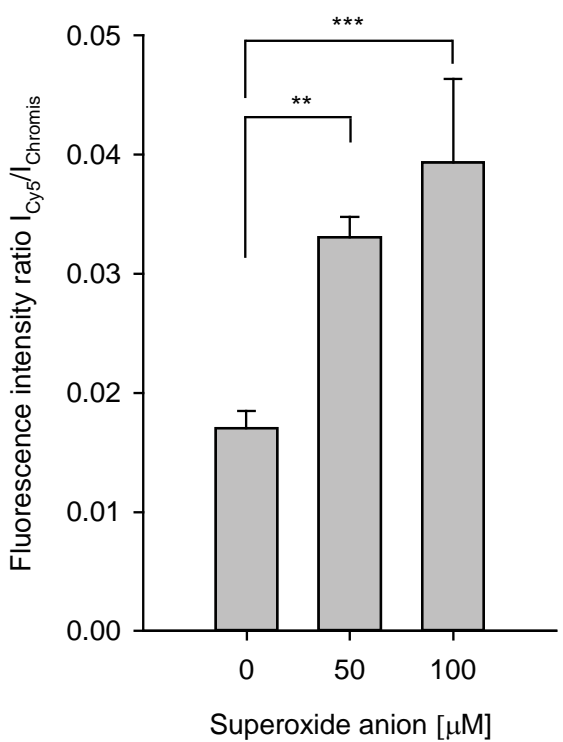

b)

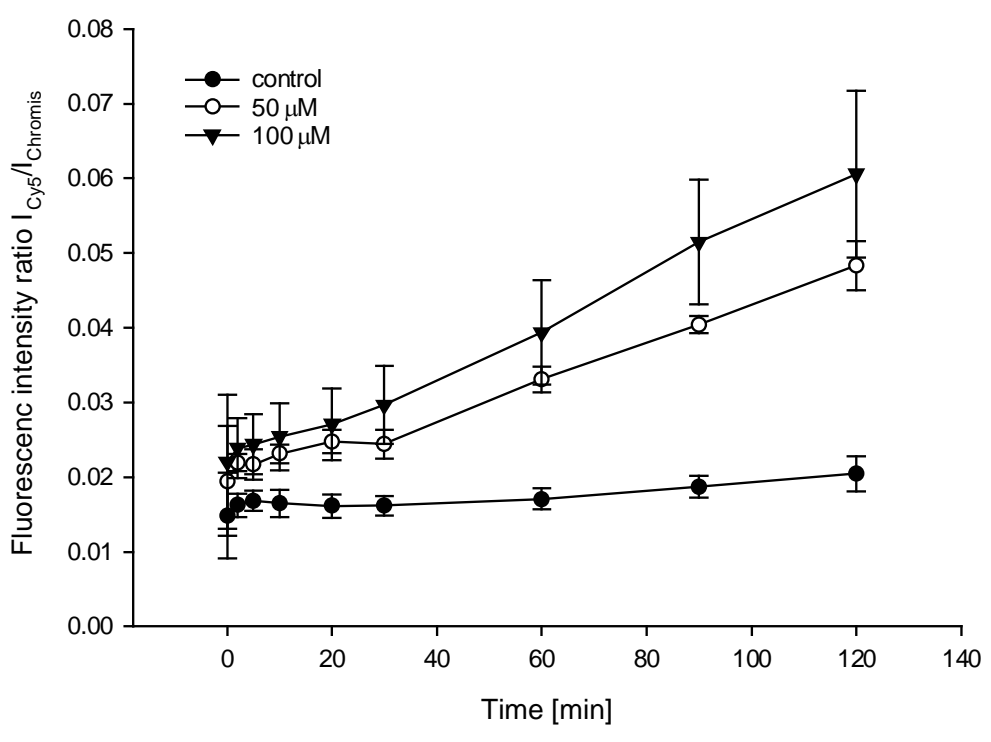

Figure 4-10: $\mathrm{O}_{2}{ }^{\circ-}$ sensing with 5 a cell-free assay at $10 \mu \mathrm{M}$ a) bar plot of the 60 min time point $($ Mean + SD), b) time course over 120 min (Mean \pm SD, $n=9)$. 


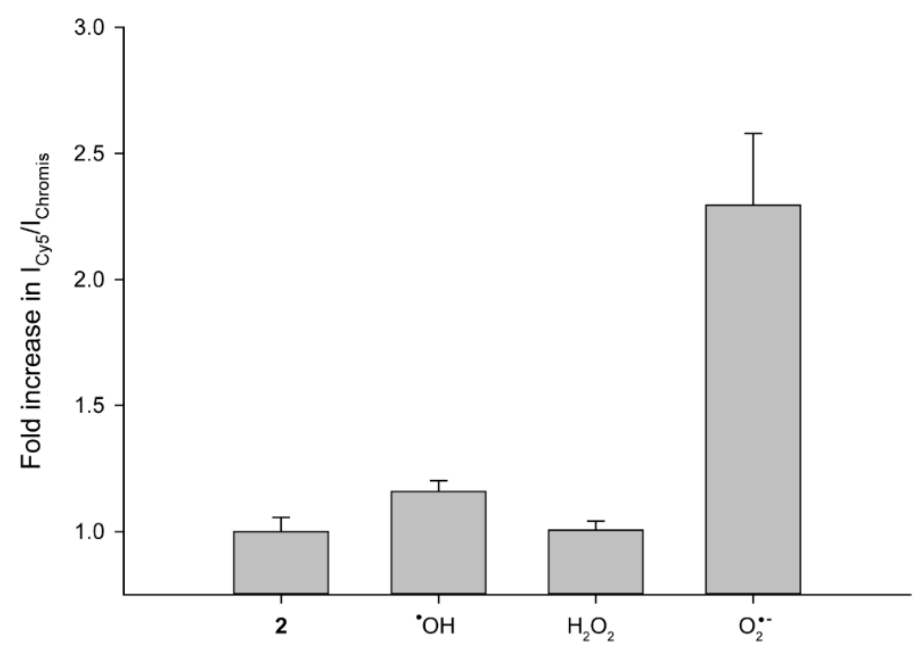

Figure 4-11: Specificity of Chromis-PGA-( $\left.\mathrm{PEG}_{10}\right)_{6}-\mathrm{HCy} 55(10 \mu \mathrm{M})$. Hydroxyl radicals ( ${ }^{\circ} \mathrm{OH}$, $200 \mu \mathrm{M}$, generated with the Fenton's reaction $\left(20 \mu \mathrm{M} \mathrm{FeSO}_{4}\right.$ and $\left.\left.200 \mu \mathrm{M} \mathrm{H}_{2} \mathrm{O}_{2}\right)\right)$ and hydrogen peroxide $\left(\mathrm{H}_{2} \mathrm{O}_{2}, 200 \mu \mathrm{M}\right)$ showed no increase in fluorescence intensity ratio even though the concentration used was the double of superoxide concentration $\left(\mathrm{O}_{2}{ }^{\circ}, 100 \mu \mathrm{M}\right.$ generated with SOTS-1). For $\mathrm{O}_{2}{ }^{--}$more than a doubling of the ratio was observed. (Mean $+\mathrm{SD}, \mathrm{n}=9$ ).

\subsection{Conclusion}

Altogether, an extracellular ratiometric probe using (H)Cy5 as ROS sensor was successfully designed. The BODIPY-analogue Chromis500 was identified as optimal reporter dye due to its resistance towards $\mathrm{NaBH}_{4}$. To minimize the FRET effect between Chromis and Cy5, discrete $\mathrm{PEG}_{10}$ were introduced to increase the distance between the dyes. Six PEG 10 units reduced the effect sufficiently and Chromis-PGA-( $\left.\mathrm{PEG}_{10}\right)_{6}-(\mathrm{H}) \mathrm{Cy} 5$ showed a negligible FRET effect. The fluorescence intensity ratio of this probe was constant up to $10 \mu \mathrm{M}$. Uptake studies in differentiated Caco- 2 cells revealed that, the introduction of a second hydrophobic fluorophore did not change the overall internalization behavior of the probe compared to PGA-Cy5 and the extracellular probe was reduced without affecting the reporter dye fluorescence. Finally, the selectivity of the coupled $(\mathrm{H}) \mathrm{Cy} 5$ shifted towards superoxide detection and it was shown, that the newly developed probe is specific for this primary ROS. The sensitivity of the ChromisPGA-(PEG 10$)_{6}-(\mathrm{H}) \mathrm{Cy} 5$ was, however, greatly reduced compared to the free $(\mathrm{H}) \mathrm{Cy} 5$. With the aim of gaining more control of the dyes (e.g. reduction of Cy5), a modular unit that allowed to combine fluorophores as last step of synthesis was introduced in a next step. 


\section{Chapter 5}

\section{Chromis-PNA:PNA-(PEG $\left.)_{10}\right)_{x}-\mathrm{HCy} 5$}

This chapter is published in:

D. Andina, D. Brambilla, N. Munzinger, J. Frei, C. Zivko, J.-C. Leroux, P. Luciani, Development of a Modular Ratiometric Fluorescent Probe for the Detection of Extracellular Superoxide, Chem. - A Eur. J. 2017, 23, 4765-4769. 


\subsection{Introduction}

With the aim of improving our first prototype Chromis-PGA-(PEG 10$)_{6}-\mathrm{Cy} 54$, the design of the probe was upgraded. The reduction of $\mathrm{Cy} 5$ to $\mathrm{HCy} 5$ without affecting the reporter dye presented a major challenge and restricted the choice of ROS-insensitive fluorophores considerably. To avoid complications during this step, a modular unit that allowed to combine the two dyes at the end of the synthetic procedure was introduced. By using complementary strands of a DNA/RNA analogue, namely peptide nucleic acid (PNA), the assembly of the final probe was achieved by a simple hybridization step (Figure 5-1). In PNA the nucleobases are connected to an uncharged polyamide backbone with the same dimensions as DNA or RNA and, therefore, allows helix formation (Figure 5-2). ${ }^{[203]}$ Due to the lack of electrostatic repulsion, PNAs form stronger Watson-Crick base pairing than DNA or RNA. The polyamide backbone stabilizes the oligomers in biological media and to enzyme degradation. ${ }^{[203-206]}$ They were first introduced in $1991^{[207]}$ and have been used in many different areas of research such as FISH probes, ${ }^{[208]}$ aptamer sensors ${ }^{[209]}$ and bio-barcode systems. ${ }^{[210,211]}$ Owing to their pseudo-peptidic backbone, PNA synthesis is compatible with SPPS and amino acids can be appended to a PNA strand. By derivatizing both strands at the 5 ' end either with a lysine residue or with the PGA-(PEG 10$)_{x^{-}}$ (H)Cy5 sensing unit (Figure 5-1, inset) a modular probe was created.

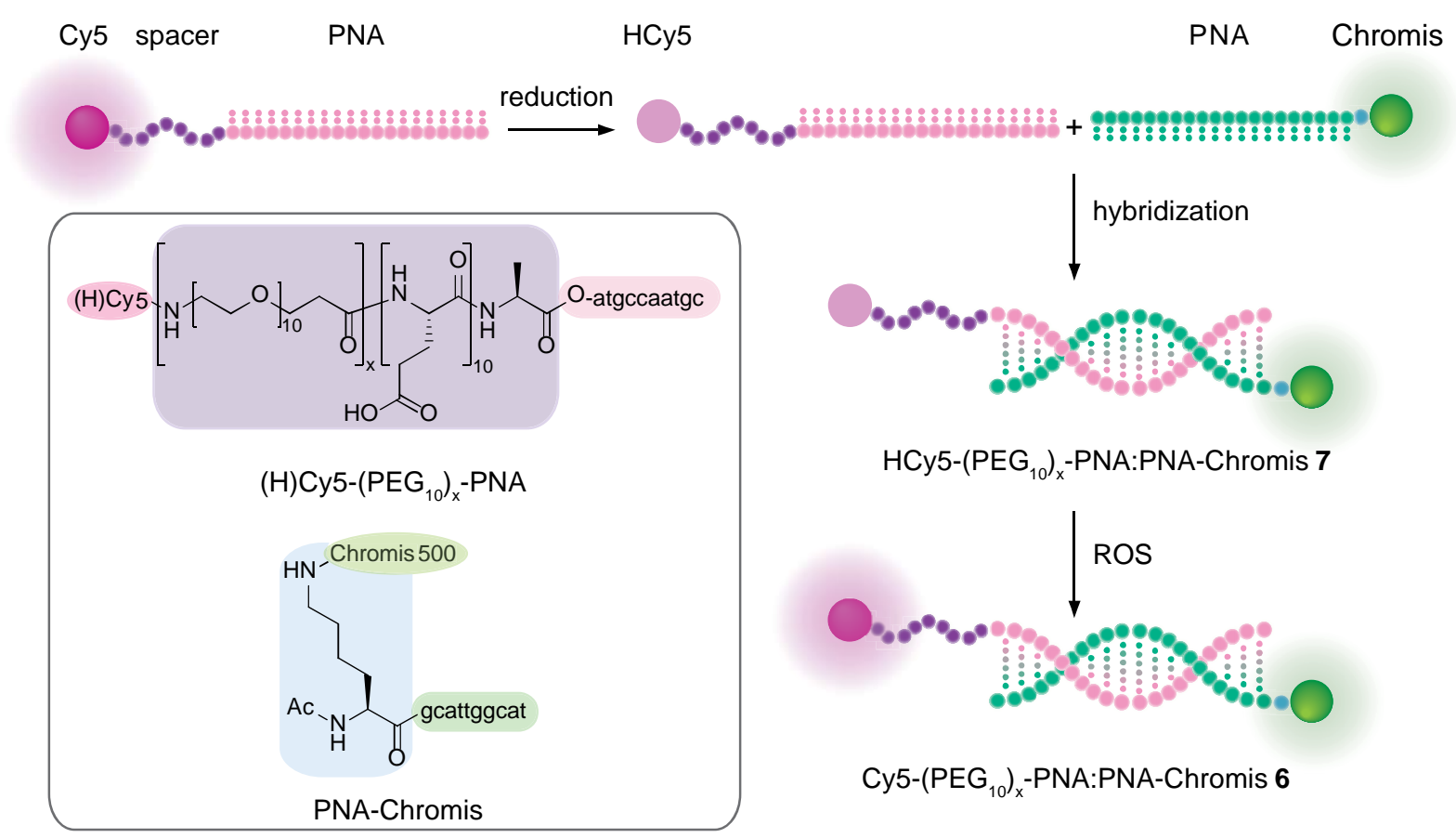

Figure 5-1: Schematic illustration of the modular ratiometric sensors, inset: Chemical structures of the building blocks. 
The optimization of the distance between the fluorophores was carried out by implementing discrete PEG $_{10}$ units as explained in Chapter 4. Hybridization of the probe was followed by reassessment of the probe's extracellular nature by uptake studies in differentiated Caco-2 monolayers. The ROS sensing capacity towards ${ }^{\circ} \mathrm{OH}$ and $\mathrm{O}_{2}{ }^{\cdot-}$ was investigated in the established cell-free assays and detection of ROS-generated in cell culture was monitored. The main findings are discussed and compared to Chromis-PGA-( $\left.\mathrm{PEG}_{10}\right)_{6}-(\mathrm{H}) \mathrm{Cy} 5$.

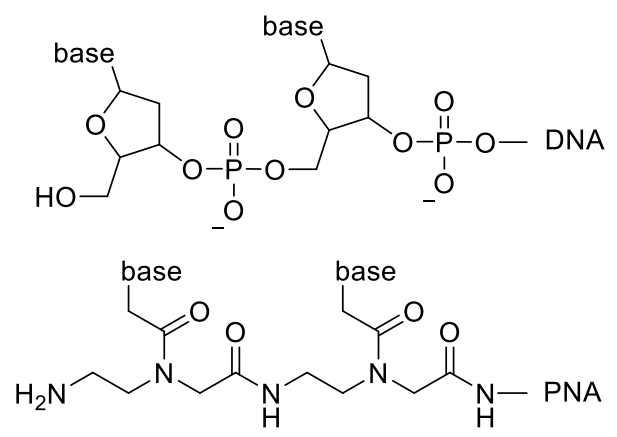

Figure 5-2: Chemical structure of the backbone of DNA and PNA. 


\subsection{Experimental Section}

\subsubsection{Materials}

Additional to the materials listed in Chapter 3 and 4 following compounds were used: Trizma ${ }^{\circledR}$ acetate, $m$-cresol and HO-Ala-2-chlorotrityl resin were purchased from Sigma Aldrich (Buchs, Switzerland). Hexafluoro-2-propanol (HFIP) was bought from Fluorochem Ltd (Derbyshire, UK). Ac-Lys-gcattggcat and resin-cgtaaccgta-O-NH 2 were purchased from Panagene (Daejong, Korea). ${ }^{[212]}$

\subsubsection{Synthesis of Chromis-PNA}

$400 \mathrm{nmol}$ Ac-Lys-gcattggcat $(1.2 \mathrm{mg}, 1 \mathrm{eq})$ were dissolved in $50 \mu \mathrm{L}$ dry DMSO. $350 \mu \mathrm{L}$ Chromis500-NHS stock solution in dry DMSO $(2 \mathrm{mg} / \mathrm{mL}, 1.2 \mu \mathrm{mol}, 3 \mathrm{eq})$ and $0.3 \mu \mathrm{L}$ DIPEA $(2 \mu \mathrm{mol}, 5 \mathrm{eq})$ were added. The mixture was shaken for $4 \mathrm{~h}$ at room temperature. After diluting with $7 \mathrm{~mL}$ of ultra-pure $\mathrm{H}_{2} \mathrm{O}$ the reaction mixture was lyophilized. The resulting yellowish solid was dissolved in water/ACN $(60: 40, v / v)$ and purified by semi-preparative HPLC (XBridge ${ }^{\mathrm{TM}}$ Prep BEH130 $\mathrm{C}_{18} 5 \mu \mathrm{m}(10 \times 150 \mathrm{~mm}), 4.4 \mathrm{~mL} / \mathrm{min}$ flow rate, isocratic with water/ACN $(+0.1$ $\%$ TFA, 60:40, $v / v) 5$ min followed by a linear gradient to 40:60 water/ACN $(v / v)$ in 20 min. The purity was verified by analytical HPLC (XBridge ${ }^{\mathrm{TM}} \mathrm{C}_{18} 5 \mu \mathrm{m} 4.6 \mathrm{~mm} \times 250 \mathrm{~mm}$ ) and a HRMS-MALDI was recorded.

\subsubsection{Synthesis of PNA-(PEG $\left.{ }_{10}\right)_{x}-\mathrm{Cy} 5$}

\section{Protected peptide-cyanine conjugate $\mathrm{HO}-\mathrm{Ala}-\mathrm{Glu}\left({ }^{t} \mathrm{Bu}\right)_{10-}\left(\mathrm{PEG}_{10}\right)_{x}-\mathrm{Cy} 5$}

Solid phase peptide synthesis (SPPS) was performed as explained in 3.2.14 and 4.2.3. Instead of a 2-chlorotrityl-Gly-OH resin a 2-chlorotrityl-Ala-OH resin was used and to obtain the fully protected peptide, the peptide was double-cleaved from the resin using 40\% HFIP in chloroform $\left(v / v, 3 \mathrm{~mL} / 100 \mathrm{mg}\right.$ resin) for $60 \mathrm{~min}$. The collected liquid was evaporated under a stream of $\mathrm{N}_{2}$ at $30{ }^{\circ} \mathrm{C}$. After dissolving in $\mathrm{ACN}: \mathrm{H}_{2} \mathrm{O}(4: 1, v / v)$ the peptide was lyophilized. This procedure was repeated once for $x=0-3$ and twice for $x=4$. The product was used without further purification. HRMS-MALDI was recorded for all protected peptide-Cy5 conjugates. 


\section{Coupling of HO-Ala-Glu ${ }_{10-P E G_{x}-C y 5}$ to resin-cgtaaccgta-O}

cgtaaccgta-O- $\mathrm{NH}_{2}$ was purchased on a Rink amide resin from Panagene. The resin was swollen in DCM for 30 min and washed 5 times with DMF using 3 resin volumes afterwards.

For $\mathbf{x}=\mathbf{0 - 3}$ : 3 eq HO-Ala-Glu $\left({ }^{t} \mathrm{Bu}\right)_{10}-\left(\mathrm{PEG}_{10}\right)_{\mathrm{x}}-\mathrm{Cy} 5$ was pre-activated with 2.9 eq HATU in DMF for 10 min. 6 eq DIPEA were added and the mixture was transferred to the resin and shaken at $700 \mathrm{rpm}$ for $2 \mathrm{~h}$. The resin was washed 5 times with DMF and 5 times with DCM using 3 resin volumes and was dried in vacuo for $16 \mathrm{~h}$.

For $\mathbf{x}=4$ : 3 eq HO-Ala-Glu $\left({ }^{t} \mathrm{Bu}\right)_{10}-\left(\mathrm{PEG}_{10}\right)_{\mathrm{x}}-\mathrm{Cy} 5$ was pre-activated with 2.9 eq HATU in DMF for 10 min. 6 eq DIPEA was added and the mixture was transferred to the resin and shaken at $1200 \mathrm{rpm}$ for $1 \mathrm{~h}$ at $75^{\circ} \mathrm{C}$ using microwave radiation. The resin was washed 5 times with DMF and 5 times with DCM using 3 resin volumes. Then it was dried in vacuo for $16 \mathrm{~h}$.

The PNA-Ala-Glu $\left({ }^{t} \mathrm{Bu}\right)_{10}-\left(\mathrm{PEG}_{10}\right)_{\mathrm{x}}-\mathrm{Cy} 5$ was cleaved from the resin using $3 \mathrm{~mL}$ TFA : $m$-cresol $(1: 4, v / v)$ for $3 \mathrm{~h}$. The liquid volume was reduced under a stream of $\mathrm{N}_{2}$ and precipitated in icecold $\mathrm{Et}_{2} \mathrm{O}$. The immediately formed blue solid was separated by centrifugation at $3000 \times g$ for $10 \mathrm{~min}$ at $4{ }^{\circ} \mathrm{C}$. The crude product was purified by semi-preparative HPLC (XBridge ${ }^{\mathrm{TM}} \mathrm{C}_{18} 5$ $\mu \mathrm{m}, 4.6 \mathrm{~mm} \times 250 \mathrm{~mm}, 1 \mathrm{~mL} / \mathrm{min}$ flow rate, isocratic with water/ACN $(+0.1 \%$ TFA, 80:20 $v / v) 5 \mathrm{~min}$ followed by a linear gradient to $20: 80$ water/ACN $(v / v)$ in $20 \mathrm{~min}$. The purity was verified by analytical HPLC.

\subsubsection{Quantification of PNA-dye conjugates}

PNA-dye conjugate concentration (CPNA) were calculated according to Equation 5-1 using the absorbance at $260 \mathrm{~nm}\left(\mathrm{~A}_{260}\right)$, the sequence specific extinction coefficient ( 2260$)$ and a correction factor for the dyes.

$$
C_{P N A}=\frac{A_{260}-A_{\text {dye } \max } \times \text { correction factor }}{\varepsilon_{260}}
$$

$\varepsilon_{260}($ Ac-Lys-gcattggcat $)=102.1 \mathrm{~mL} /(\mu \mathrm{mol} \times \mathrm{cm})$

$\varepsilon_{260}(\mathrm{O}-\operatorname{atgccaatgc})=101.9 \mathrm{~mL} /(\mu \mathrm{mol} \times \mathrm{cm})$

Cy5: correction factor $=0.05, A_{\text {dye } \max }=649 \mathrm{~nm}$

Chromis500: correction factor $=0.01$, Adye $\max =496 \mathrm{~nm}$ 


\subsubsection{Hybridization}

Equimolar amounts of the PNA-dye conjugates were mixed in PBS buffer $(\mathrm{pH}=7.4)$ and heated to $90{ }^{\circ} \mathrm{C}$ for $1 \mathrm{~min}$. The sample was cooled down slowly to $4{ }^{\circ} \mathrm{C}$. The hybridization was visualized by a native PAGE (18\% acryl amide gel, running buffer: Tris-acetate-EDTA buffer (TAE; $40 \mathrm{mM}$ Tris-acetate, $1 \mathrm{mM}$ EDTA, $\mathrm{pH}=8.0$ ). The gel was imaged with an IVIS instrument at the wavelength of the dyes used (Chromis500: $470 \mathrm{~nm} / 530 \mathrm{~nm}$; Cy5: $650 \mathrm{~nm} / 700$ $\mathrm{nm})$.

\subsubsection{Energy transfer measurement}

The different Chromis-PNA:PNA-( $\left.\mathrm{PEG}_{10}\right)_{0-4}-\mathrm{Cy} 5$ were hybridized and the native PAGE was run as described above. The band of the hybridized probe was excised from the gel and transferred to a $0.5 \mathrm{~mL}$ Eppendorf tube with a hole. This was placed in a $1.5-\mathrm{mL}$ Eppendorf tube and centrifuged for $10 \mathrm{~min}$ at $18,210 \times g$. The crushed gel was transferred to a glass vial and the product was extracted in $100 \mu \mathrm{L}$ running buffer for $16 \mathrm{~h} .80 \mu \mathrm{L}$ sample were transferred to a multi-well quartz plate (Hellma Analytics). Energy transfer between the dyes was measured by excitation at the lower wavelength (for Chromis500/Cy5 $\lambda_{\mathrm{ex}}=470 \mathrm{~nm}$ ) and recording of a fluorescence scan (for Chromis500/Cy5 from $490 \mathrm{~nm}-800 \mathrm{~nm}$ ) on a plate in a black multiwell quartz plate (Hellma Analytics). The distance between the dyes was calculated as described 4.2.5.

\subsubsection{Reduction of the cyanine dye}

PNA-( $\left.\mathrm{PEG}_{10}\right)_{3}-\mathrm{Cy} 5$ was dissolved in $\mathrm{H}_{2} \mathrm{O}$ and a bubbling $1 \mathrm{mg} / \mathrm{mL} \mathrm{NaBH}_{4}$ solution in $\mathrm{MeOH}$ was slowly added to the blue dye solution. The loss of blue color indicated that the reduction occurs. The fluorescence intensity was measured and the reduction was considered complete if the value obtained was $<1000\left(\lambda_{\mathrm{ex}}=620, \lambda_{\mathrm{em}}=665\right.$, gain 150$)$.

\subsubsection{Re-oxidation with the Fenton's reagent}

$\mathrm{HO}^{\bullet}$ sensing experiments were carried out as described in 3.2.4. 


\subsubsection{Re-oxidation with SOTS-1 ${ }^{[193,194]}$}

$\mathrm{O}_{2}{ }^{\bullet-}$ sensing experiments were carried out as described in 3.2.5.

\subsubsection{Uptake in differentiated Caco-2 monolayers}

Uptake studies were carried out as explained in 4.2.10.

\subsubsection{Stimulation of Caco-2 cells $^{[213]}$}

ROS production was induced and monitored as stated in 3.2.10

\subsubsection{Statistical Analysis, Coefficient of variation}

Results were analysed and the coefficient of variation was calculated as described in 4.2.11. 


\subsection{Results and Discussion}

\subsubsection{Synthesis and optimization of PNA-dye conjugates}

To gain a better control over the fluorophores, a double stranded PNA was implemented in the linker, thereby creating two building blocks each of them bearing one dye. To simplify the synthesis of the PNA-dye conjugates, the PNA strands were inserted between the lysine residue and the PGA-( $\left.\mathrm{PEG}_{10}\right)_{\mathrm{x}}-(\mathrm{H}) \mathrm{Cy} 5$, leading to a Lys-PNA and a PNA-peptide-Cy5 conjugate (Figure 5-1 inset). The PNA sequence used in this work consisted of ten nucleotides and was shown to have a high melting temperature with its complementary DNA strand $\left(\mathrm{T}_{\mathrm{m}}=333.2-\right.$ $334.6 \mathrm{~K}) .{ }^{[212]}$ The primary amine of the lysine-modified strand (Ac-Lys-gcattggcat, Lys-PNA) underwent reaction with NHS-activated Chromis under basic conditions in solution resulting in PNA-Chromis (Figure 5-3 a). After semi-preparative HPLC purification, PNA-Chromis was characterized with analytical HPLC, MALDI-MS (Figure A32-33) and spectrophotometric methods (Figure 5-4).

a)

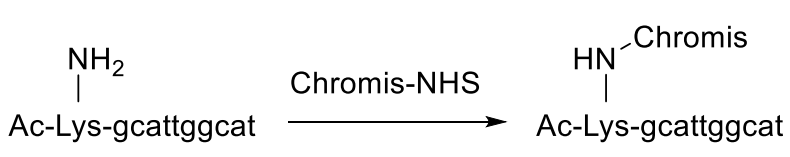

b)
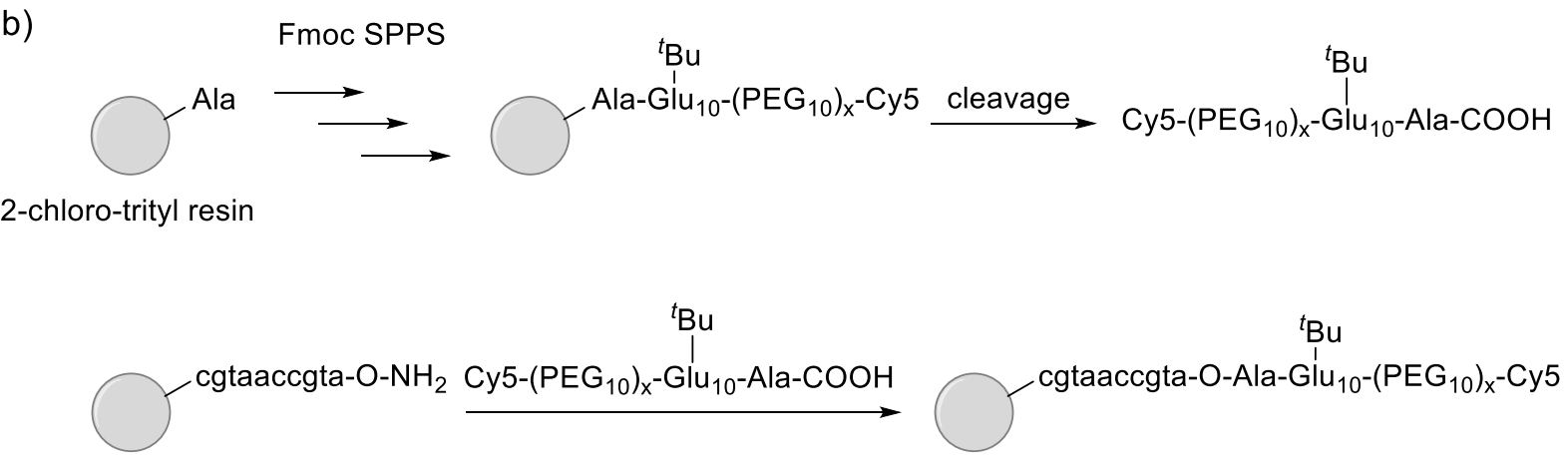

Rink amide resin

cleavage

Cy5-PEG - Glu $_{10}-$ Ala-O-atgccaatgc

Figure 5-3: a) Synthesis of PNA-Chromis b) Synthesis of the protected Ala-Glu $\left({ }^{t} \mathrm{Bu}\right)_{10}-$ $\left(\mathrm{PEG}_{10}\right)_{\mathrm{x}}-\mathrm{Cy} 5(\mathrm{x}=0-4)$ and coupling of the protected peptide-Cy5 to PNA-resin. 


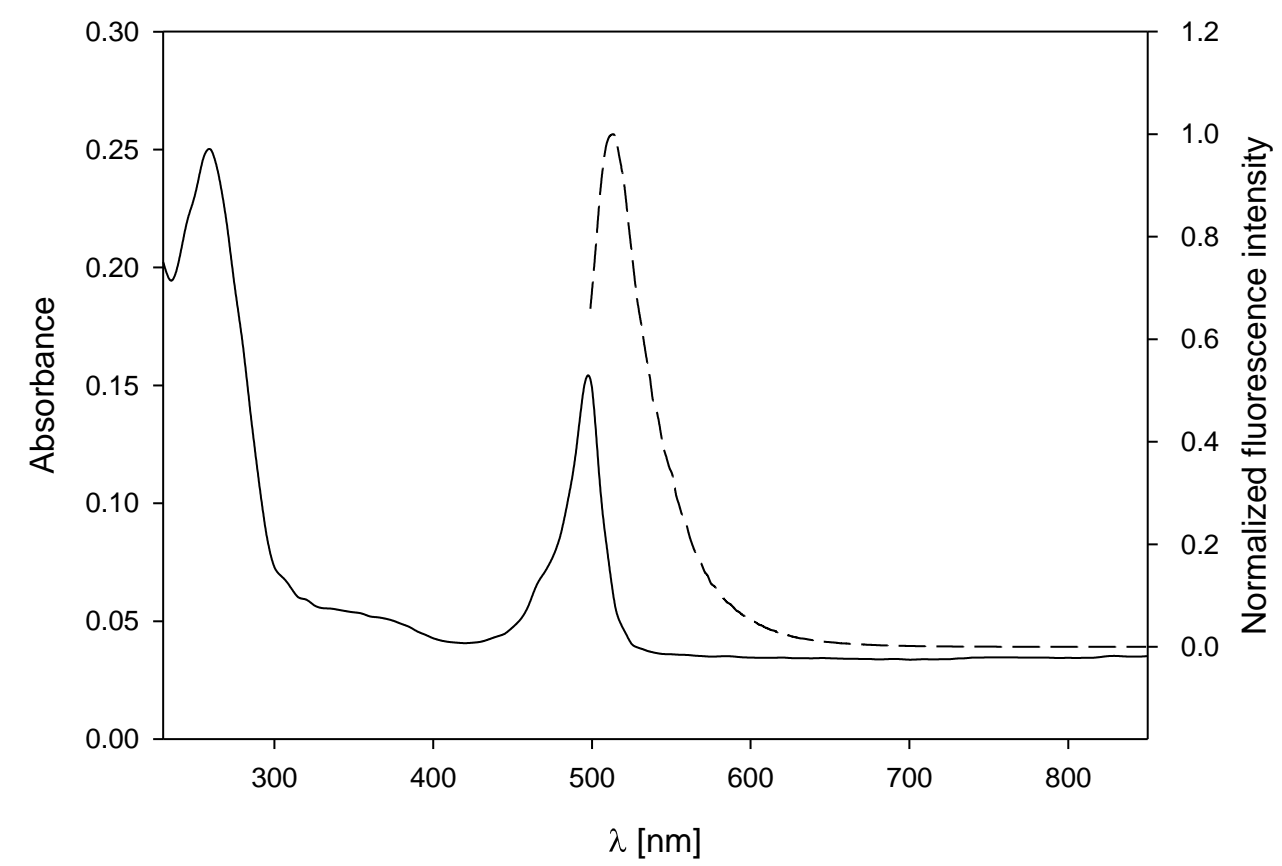

Figure 5-4: Absorbance (solid line, left axis) and fluorescence emission ( $\lambda_{\mathrm{ex}}=470 \mathrm{~nm}$, dashed line, right axis) scan of PNA-Chromis.

The complementary PNA strand was directly modified at the N-terminus on the Rink amide resin with PGA-(PEG 10$)_{x}-\mathrm{Cy} 5$ moiety. To couple the peptide to the N-terminus of PNA-resin, the protected peptide-Cy5 was synthesized by manual SPPS (Figure 5-3 b) using the same synthesis conditions as described in Chapter 3 and 4. To obtain the fully protected peptide cleavage conditions from the 2-chlorotrityl resin were changed to 40\% HFIP. Interestingly, the loss of exactly one tert-butyl protecting group was observed, when glutamic acid was directly attached to the 2-chlorotrityl resin, but by introducing the helix inducing alanine residue this issue could be solved. ${ }^{[214]}$ The fully protected peptide-Cy5 was analyzed with MALDI-MS (Figure A34-38) and to study the impact of spacer length on FRET efficiency, the number of $\mathrm{PEG}_{10}$ units was varied from zero to four (Figure 5-3 b, $\mathrm{x}=0-4$ ). The five protected peptidic units were reacted with the PNA linked to a resin using common coupling conditions at room temperature for $\left(\mathrm{PEG}_{10}\right)_{0-3}$ and microwave assisted conditions at $70{ }^{\circ} \mathrm{C}$ for $\left(\mathrm{PEG}_{10}\right)_{4}$. The PNApeptide-Cy5 conjugates (PNA-(PEG 10$\left.)_{x}-\mathrm{Cy} 5\right)$ were purified and analyzed as PNA-Chromis (Figure A39-48). Annealing of the strands resulted in the ratiometric sensor in the unreduced form and was verified by a native PAGE (Figure 5-5) using an excess PNA-Chromis. Due to a lack of charge, PNA-Chromis did not show any motility on the gel and is visible as blue band. The single strands of PNA-( $\left.\mathrm{PEG}_{10}\right)_{0-4}-\mathrm{Cy} 5$ move more than the hybridized probes 
Chromis-PNA:PNA-(PEG10)0-4-Cy5. A molecular weight dependent motility of the single strand but also of the hybridized compounds analyzed.

Gel 1
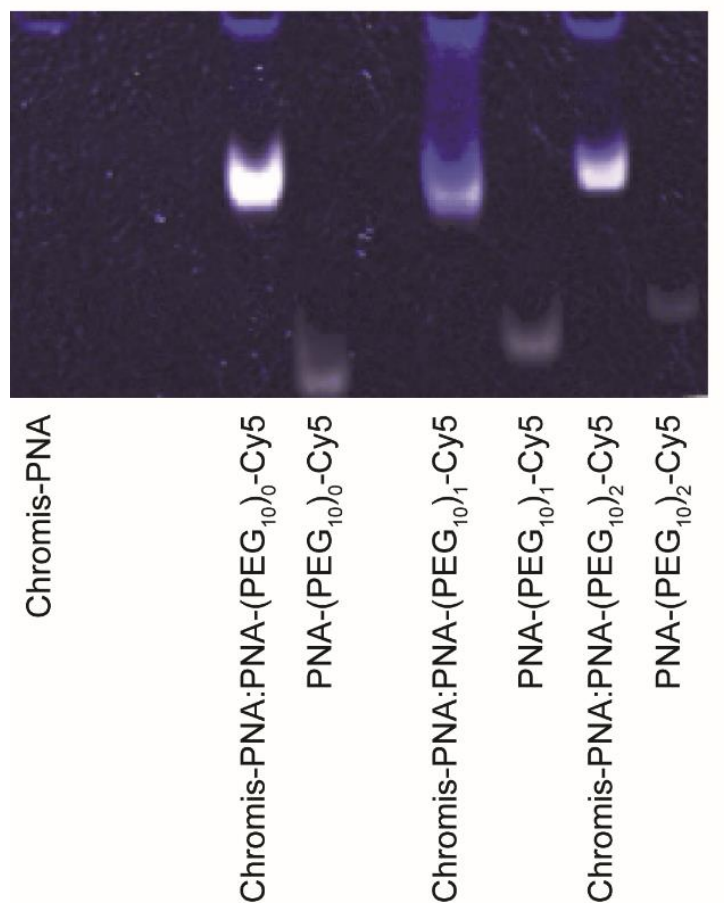

Gel 2

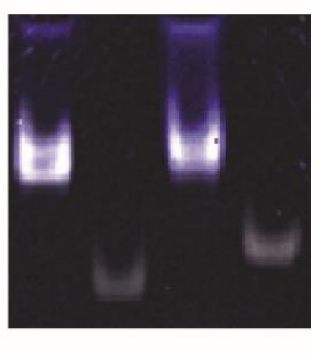

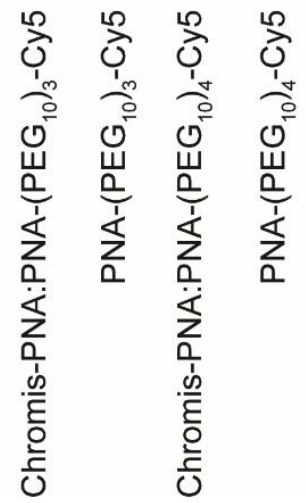

Figure 5-5: Motility of Chromis-PNA:PNA-(PEG 10$)_{x}-C y 5$ on a native PAGE (loading $10 \mu \mathrm{M}$ ). Excess PNA-Chromis is visible as a first band in all hybridized probes and in the lane of ChromisPNA alone. The single PNA- $\left(\mathrm{PEG}_{10}\right)_{\mathrm{x}} \mathrm{Cy} 5$ strands migrate more than the hybridized probes Chromis-PNA:PNA-( $\left.\mathrm{PEG}_{10}\right)_{\mathrm{x}} \mathrm{Cy} 5$ due to their lower molecular weight. The motility of the single strands PNA-( $\left.\mathrm{PEG}_{10}\right)_{\mathrm{x}}-\mathrm{Cy} 5$ and the hybridized probes Chromis-PNA:PNA-( $\left.\mathrm{PEG}_{10}\right)_{\mathrm{x}}-\mathrm{Cy} 5$ are molecular weight dependent. Blue: Chromis, grey: Cy5, white: overlay.

To study the effect of spacer length on FRET efficiency in these probes, they were purified with a native PAGE. The band corresponding to the hybridized probe was excised from the gel and extracted overnight. The energy transfer was measured by excitation of Chromis at $470 \mathrm{~nm}$ and recording a fluorescence emission scan. The observed differences between the various spacer length were small (Figure 5-6). Calculation of the approximate distance revealed that the distance between the dyes of all PNA probes was bigger than the one observed in ChromisPGA-(PEG10)6-Cy5 4 (72-80 ̊ vs. $71 \AA$, Figure 5-6). Chromis-PNA:PNA-(PEG10)3-Cy5 6 exhibited the smallest energy transfer and was, therefore, used in all further experiments.

Characterization of Cy5-( $\left.\mathrm{PEG}_{10}\right)_{3}$-PNA was carried out as for all the other strands with HPLC and MALDI-MS (Figure A45-46). Spectrofluorometric analysis of the assembled probe Cy5( $\left.\mathrm{PEG}_{10}\right)_{3}$-PNA:PNA-Chromis is presented in Figure 5-7. 


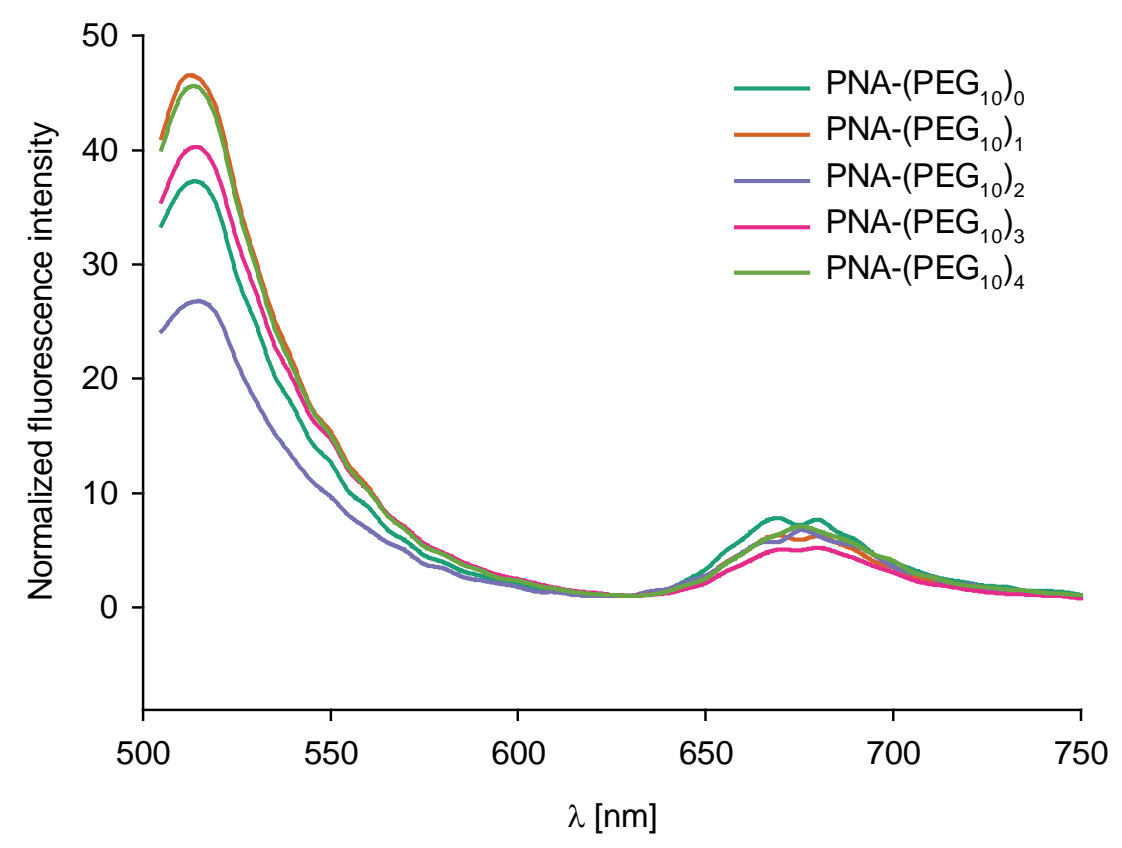

\begin{tabular}{|c|c|c|}
\hline Chromis-PNA:PNA-(PEG $\left.{ }_{10}\right)_{x}-C y 5$ & $E_{\text {rel }}$ & $\mathbf{R}[\AA ̊]$ \\
\hline Chromis-PNA:PNA-(PEG $\left.{ }_{10}\right)_{0}-\mathrm{Cy} 5$ & 0.171 & 74 \\
\hline Chromis-PNA:PNA-(PEG $\left.{ }_{10}\right){ }_{1}-\mathrm{Cy} 5$ & 0.119 & 79 \\
\hline Chromis-PNA:PNA-(F & 0.190 & 72 \\
\hline Chromis-PNA:PNA-(PEG $\left.{ }_{10}\right)_{3}-\mathrm{Cy} 5$ & 0.115 & 80 \\
\hline Chromis-PNA:PNA-(PEG 10$)_{4}-\mathrm{Cy} 5$ & 0.128 & 78 \\
\hline
\end{tabular}

Figure 5-6: Effect of spacer length on FRET efficiency, $\lambda_{\mathrm{ex}}=470 \mathrm{~nm}$. The effect of the spacer length on the FRET efficiency was small. Only a minor improvement from PNA-( $\left.\mathrm{PEG}_{10}\right)_{0}$ to PNA-( PEG $\left._{10}\right)_{3}$ was observed. Black box: Calculation of the length of the spacer unit using the FRET scans. 


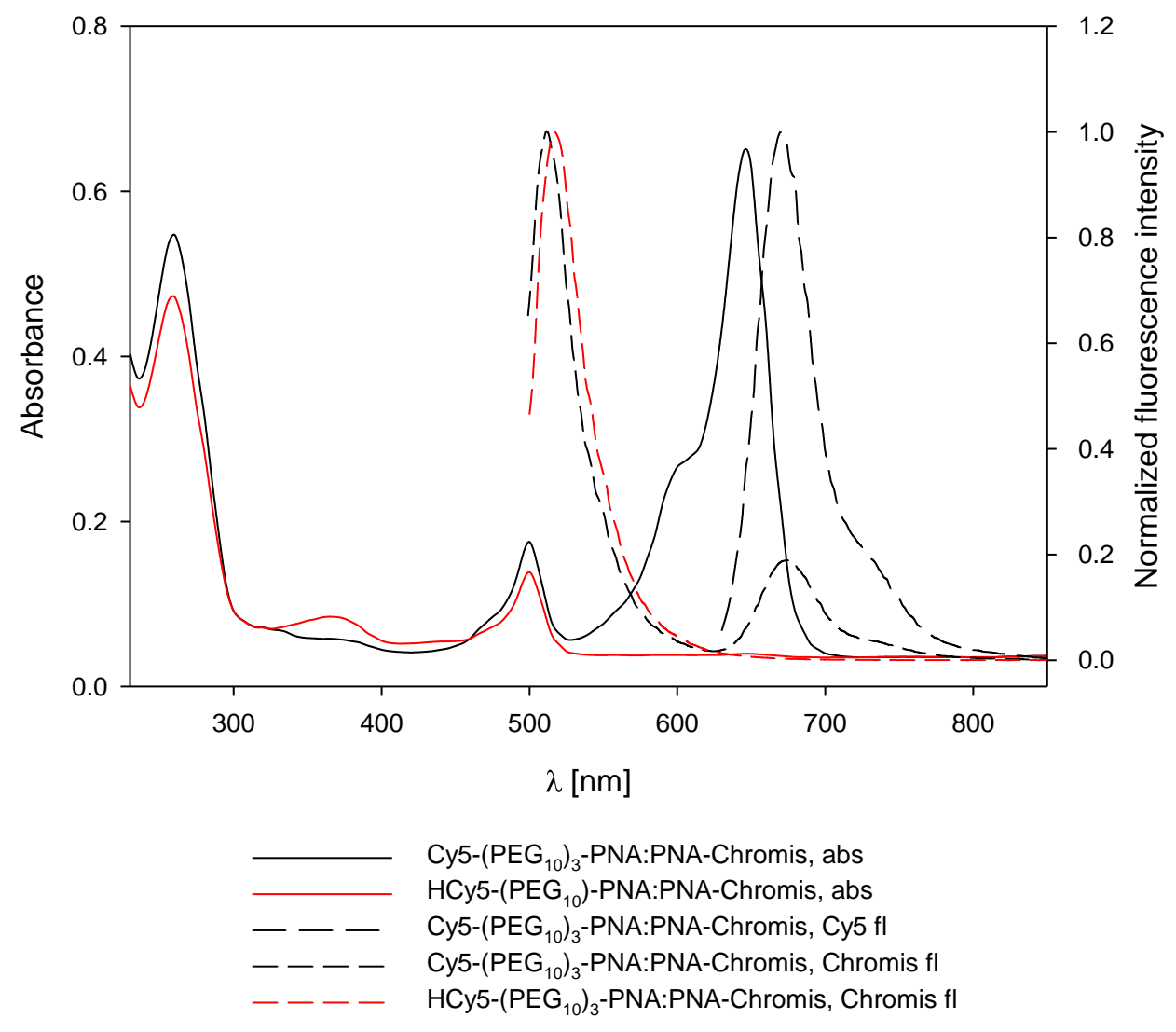

Figure 5-7: Absorbance scans of Cy5-(PEG 10$)_{3}-\mathrm{PNA}$ :PNA-Chromis 6 (black solid line, left axis) and 7 (red solid line, left axis) and fluorescence emission scans of Cy5-(PEG $\left.{ }_{10}\right)_{3}-\mathrm{PNA}$ :PNAChromis 3 (dashed black lines, right axis) and HCy5-(PEG $\left.{ }_{10}\right)_{3}$-PNA:PNA-Chromis 6 (dashed red line, right axis). Chromis $\lambda_{\mathrm{ex}}=470 \mathrm{~nm}, \mathrm{Cy} 5 \lambda_{\mathrm{ex}}=620 \mathrm{~nm}$. 


\subsubsection{Uptake studies of Chromis-PNA:PNA-(PEG 10$)_{3}-\mathrm{Cy5}$}

The uptake of Chromis-PNA:PNA-(PEG 10$)_{3}-\mathrm{Cy} 5$ in differentiated Caco-2 cells was assessed by incubating the free dyes and 6 for $1 \mathrm{~h}$, followed by flow cytometer analysis. The internalization of 6 was significantly reduced compared with the free dyes Cy5 and Chromis (Figure 5-8), confirming that the probe design still prevents uptake by cells, although the overall hydrophilicity of the sensor was altered by introducing the relatively hydrophobic PNA moiety.
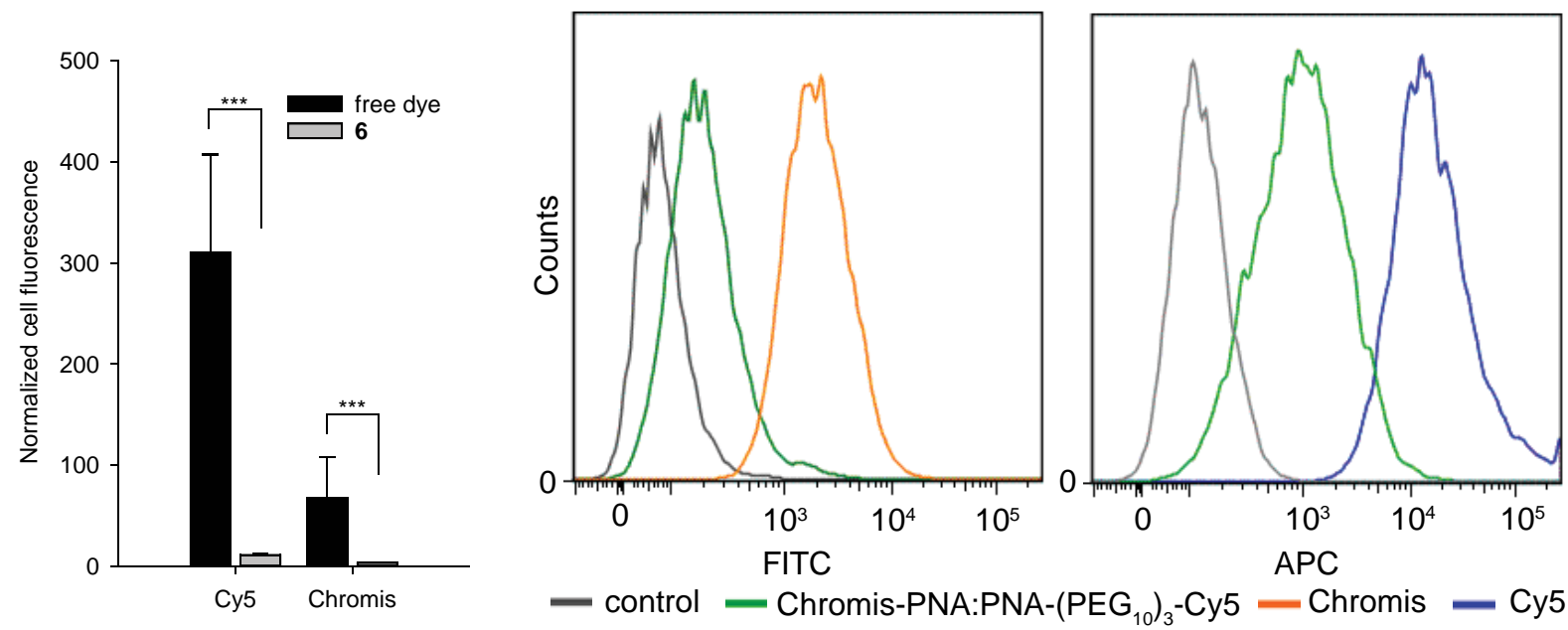

Figure 5-8: Representative flow cytometry diagrams of the uptake of Chromis-PNA:PNA$\left(\mathrm{PEG}_{10}\right)_{3}-\mathrm{Cy} 53$ in differentiated Caco-2 monolayers after $1 \mathrm{~h}$ of incubation at $37{ }^{\circ} \mathrm{C}$. Left: filter for Cy5, right: filter for Chromis. Chromis-PNA:PNA-( $\left.\mathrm{PEG}_{10}\right)_{3}-\mathrm{Cy} 56$ shows a lower intensity for both filters than the free dyes. 


\subsubsection{Reduction and annealing of Chromis-PNA:PNA-(PEG 10$)_{3}-\mathrm{HCy}_{5}$}

To verify the successful annealing of the two strands and to show that it is not affected by the reduction of the cyanine dye, a native PAGE was used to analyze the reduced 7 and the nonreduced sensor 6 (Figure 5-9). The yellow band, being an overlay of green (Chromis) and red (Cy5), confirmed annealing of the modified strands. The lack of other bands or a band corresponding to Cy5-( $\left.\mathrm{PEG}_{10}\right)_{3}$-PNA (red band) showed that most of the probe was successfully hybridized in a 1:1 ratio and no undesired structures are built (e.g. triplex). Chromis-PNA does not have negative charges and, thus, did not show any electrophoretic mobility. The same position of the green (4) and yellow band (3) indicated that both imaging probes have similar molecular weights and negative charges, which proved that the reduction of Cy5 to HCy5 did not affect the hybridization of the ratiometric PNA probe.

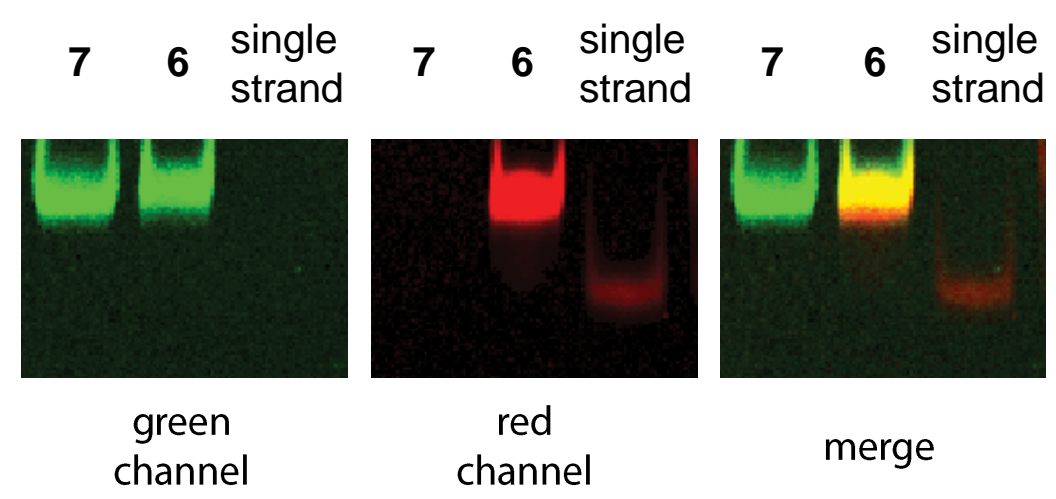

Figure 5-9: Native PAGE of Chromis-PNA:PNA-(PEG 10$)_{3}-\mathrm{HCy} 5$ 7, Chromis-PNA:PNA$\left(\mathrm{PEG}_{10}\right)_{3}-\mathrm{Cy} 56$ and Cy5-( $\left.\mathrm{PEG}_{10}\right)_{3}$-PNA (single strand), Chromis: green, Cy5: green, overlay: yellow.

\subsubsection{Sensing of $\mathrm{HO}^{\circ}$ and $\mathrm{O}_{2}{ }^{--}$radicals in cell free assays}

In analogy to Chromis-PGA-(PEG10)6-(H)Cy5, detection of $\mathrm{HO}^{\bullet}$ was attempted. A maximal increase in fluorescence intensity ratio of 1.2-fold was observed, that could not even be detected after $2 \mathrm{~h}$ (Figure 5-10). The $\mathrm{O}_{2}{ }^{*-}$ generation by SOTS-1 was monitored at 50 and $100 \mu \mathrm{M}$ and revealed a 1.3-fold and 2.2-fold increase, respectively, after $1 \mathrm{~h}\left(\mathrm{c}_{\text {probe }}=10 \mu \mathrm{M}\right.$, Figure 5-11). The CV was low for both concentrations (4\% and 3\%). These results suggest, that the sensing behavior compared with Chromis-PGA-( $\left.\mathrm{PEG}_{10}\right)_{3}-(\mathrm{H}) \mathrm{Cy} 5$ were not affected by the introduction of the modular unit and that also for the modular ratiometric prototype, the sensing behavior was shifted towards specific $\mathrm{O}_{2}{ }^{*-}$ detection. 

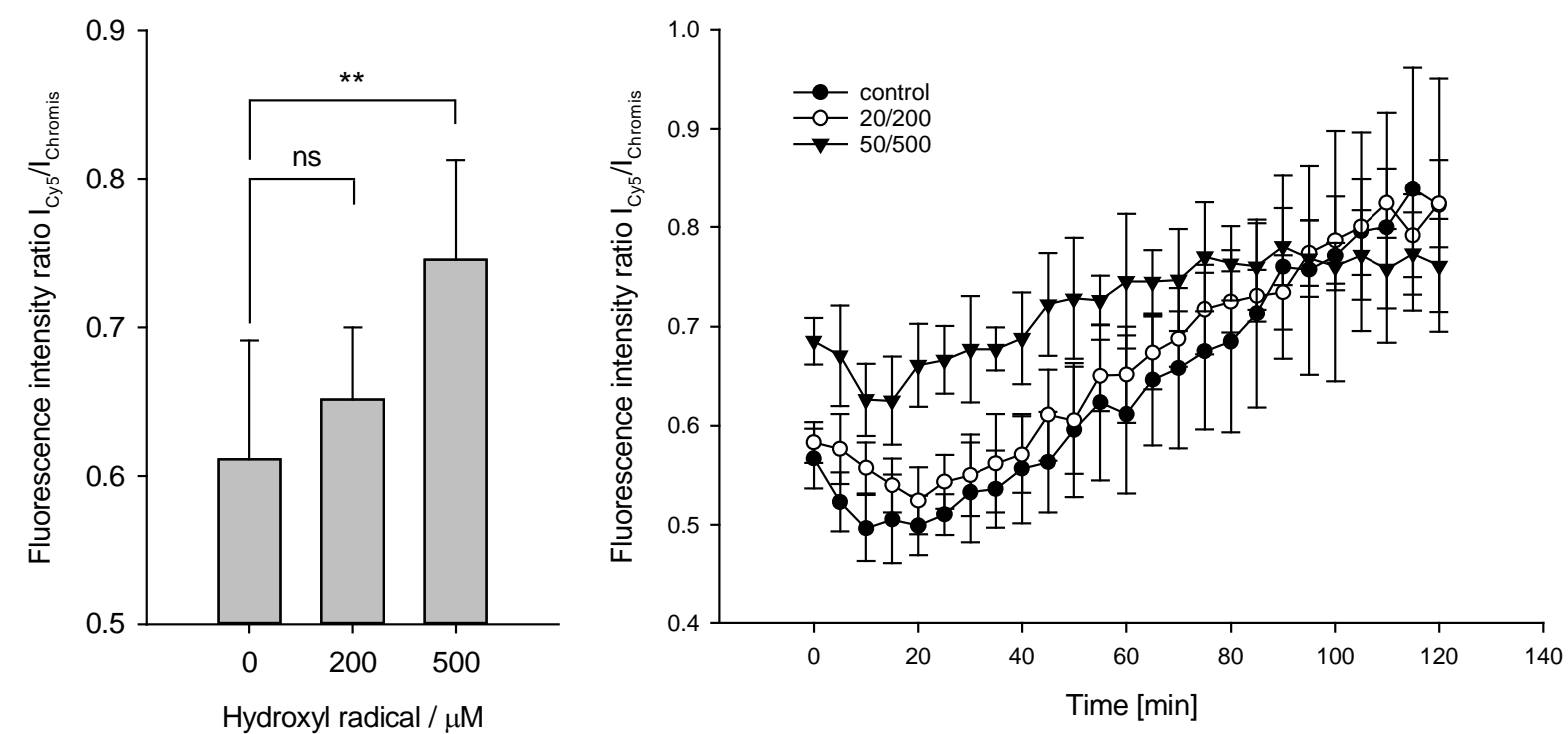

Figure 5-10: Sensing of $\mathrm{HO}^{\bullet}$ with Chromis-PNA:PNA-( $\left.\mathrm{PEG}_{10}\right)_{3}-\mathrm{HCy} 57$ with the Fenton's reaction $\left(\left[\mathrm{FeSO}_{4}\right] /\left[\mathrm{H}_{2} \mathrm{O}_{2}\right]\right)$. The bar plot presents the increase at $\mathrm{t}=60 \mathrm{~min}$. The sensing was monitored for $2 \mathrm{~h}$. Legend: $\left[\mathrm{FeSO}_{4}\right] /\left[\mathrm{H}_{2} \mathrm{O}_{2}\right]$ in $\mu \mathrm{M}$. (Mean $\pm \mathrm{SD}, \mathrm{n}=6$ ).
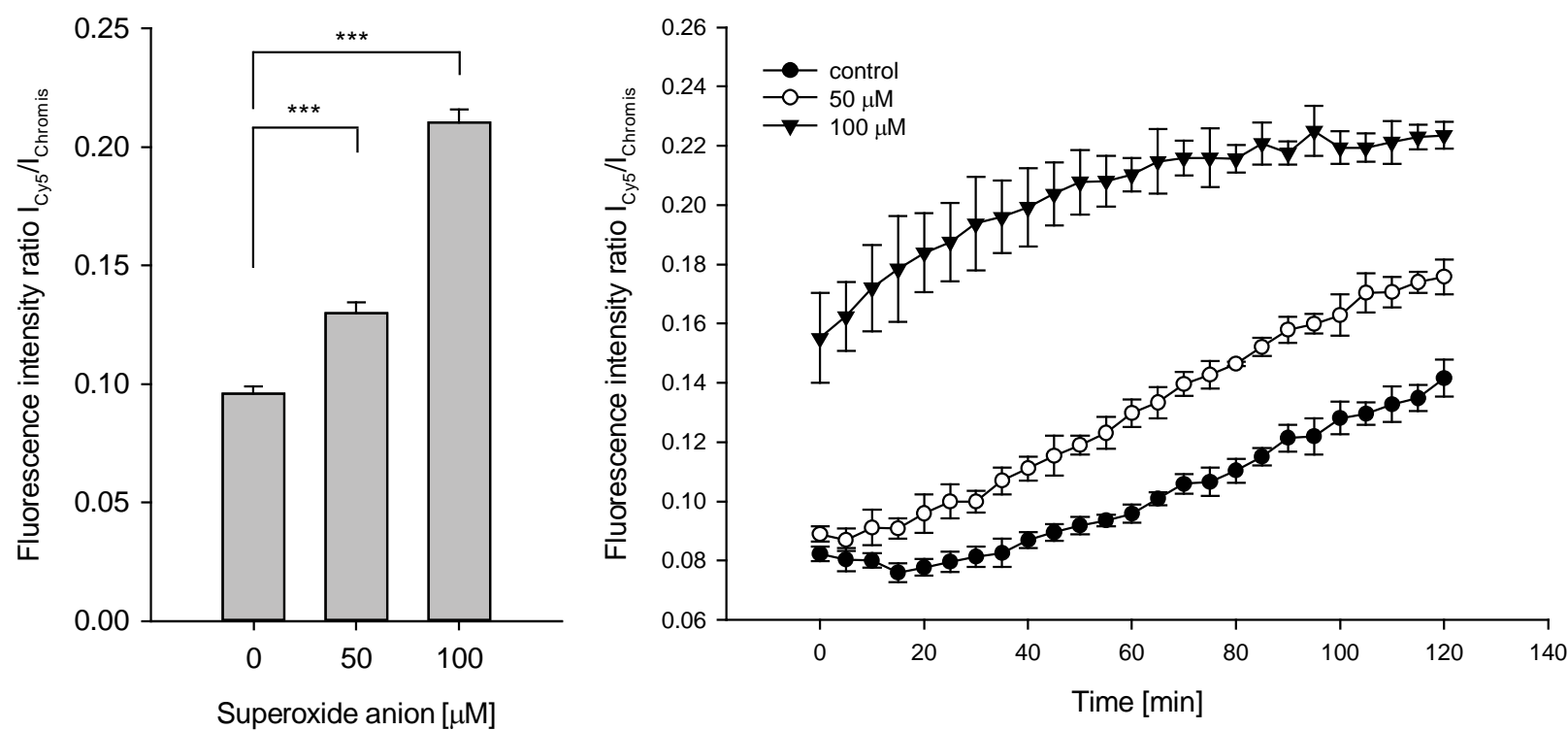

Figure 5-11: Sensing of $\mathrm{O}_{2}{ }^{--}$with Chromis-PNA:PNA-(PEG 10$)_{3}-\mathrm{HCy} 57$ monitored over $2 \mathrm{~h}$. A steady increase of $\mathrm{O}_{2}{ }^{--}$was detected, showing that variation of concentration can be monitored for at least $2 \mathrm{~h}$. The bar plot shows the increase at $\mathrm{t}=60 \mathrm{~min}$. Control: 4 . (Mean $\pm \mathrm{SD}, \mathrm{n}=6$ ). 


\subsubsection{Stimulation of non-differentiated Caco-2 cells}

As $\mathrm{O}_{2}{ }^{*-}$ detection was possible, oxidative stress sensing in vitro was investigated using 2,2'azobis(2-amidinopropane) dihydrochloride (AAPH) and $\mathrm{H}_{2} \mathrm{O}_{2}$-stimulated Caco-2 cells. AAPH showed a 1.4-fold increase after $1 \mathrm{~h}$ with a $\mathrm{CV}$ of $6 \%$. A steady increase was observed reaching the same level as $\mathrm{H}_{2} \mathrm{O}_{2}$-stimulated cells after $2 \mathrm{~h}$. For $\mathrm{H}_{2} \mathrm{O}_{2}$-stimulated cells a doubling of the ratio was observed immediately with a $\mathrm{CV}$ of $10 \%$ (Figure 5-12). Overall, the sensing capacity of 7 was as good as the covalently linked ratiometric probe $\mathbf{5}$ in the cell-free assays, which corroborates that the design of modular platform increases the flexibility of the sensor with equal ROS detection ability. Additionally, 7 was successfully used to detect extracellular oxidative stress in cell culture.
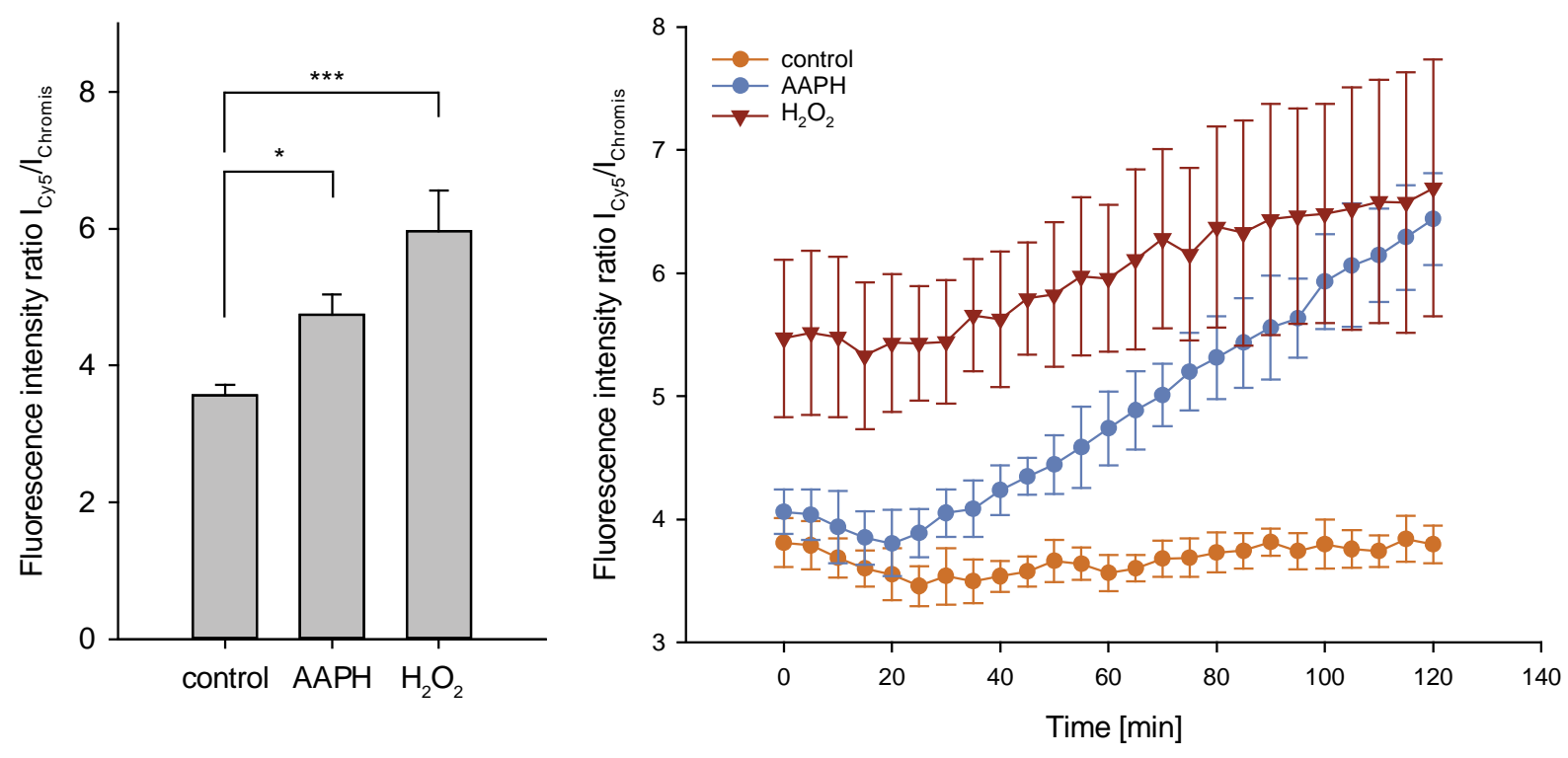

Figure 5-12: Sensing with Chromis-PNA:PNA-( $\left.\mathrm{PEG}_{10}\right)_{3}-\mathrm{HCy} 57$ of oxidative stress generated by stimulated Caco-2 cells monitored over $2 \mathrm{~h}$. The bar plot represents the increase at $\mathrm{t}=60 \mathrm{~min}$. (Mean \pm SD, $n=9$ ). 


\subsection{Conclusion}

In summary, we have modified the ratiometric probe described in Chapter 4 by implementing a PNA double strand to obtain a modular ROS sensor that can be assembled under mild conditions just before the sensing experiments. The number of $\mathrm{PEG}_{10}$ units did not have a significant effect on FRET efficiency, that was very low in general and smaller than observed for Chromis-PGA-(PEG10)6-(H)Cy5. Chromis-PNA:PNA-(PEG 10$)_{3}-(\mathrm{H}) \mathrm{Cy} 5$ was selected for further experiments, due to its favorable characteristics and its uptake in differentiated Caco-2 cell monolayers was studied. The internalization of the modular probe was greatly reduced compared to the uncoupled dyes ( $>20$-fold decrease). As for Chromis-PGA-(PEG 10$)_{6}-(\mathrm{H}) \mathrm{Cy} 5$ sensing selectivity was shifted towards $\mathrm{O}_{2}{ }^{--}$and a was not significantly altered by the modular PNA unit introduced. Additionally, ROS detection in cell culture was achieved with nondifferentiated Caco-2 cells. Even though the sensitivity of our modular ratiometric ROS-probe is lower when compared to $\mathrm{HCy} 5$ in cell-free as well as in cell-based assays, $\mathrm{O}_{2}{ }^{\circ-}$ detection was exemplified and was, indeed, similar to the commercially available hydrocyanines. ${ }^{[215]}$ This modular platform can be used to build-up a library with other reference dyes or sensor fluorophores and can be combined flexibly by a simple hybridization step. 
Chapter 6

Conclusion and Outlook 
ROS are short-lived molecules that are endogenously produced, for example by neutrophils during the respiratory burst in host defense. In this first-line defense mechanism of the innate immune response, ROS exert important antimicrobial activities against intruders and the dysregulation of these events can have deleterious effects on health. Over the last twenty years, the spectrum of action of these transient molecules has been broadened with an ever-increasing number of signaling functions in stem cell differentiation, cancer and ageing, among others. The growing interest in ROS calls for reliable and specific detection methods to study them, which, due to the molecules' transient nature and similar chemical reactivity, imposes a substantial challenge.

The development of fluorescent probes for the detection of ROS has received considerable attention, with new sensors being constantly introduced. Although the design of most probes have aimed at ROS imaging in the intracellular space, selective detection in the extracellular milieu can also give important insights, as it has been shown that the function of ROS can vary depending on their location (e.g. in tumorigenesis). Thus, the study presented in this thesis aimed at designing an extracellular fluorescent ROS-sensor that addressed some of the usual obstacles encountered by fluorescent probes. To minimize signal fluctuations and to eliminate artefacts, a ratiometric probe was created by linking a ROS-indicator and a reference dye with a charged bio-polymeric moiety. The linker enabled the modification of the probe's properties (e.g. uptake by cells) and the use of an existing indicator for intracellular ROS detection.

As presented in Chapter 3, our choice of hydrocyanines as model ROS-sensor was based on promising reports in literature showing that not only a high fluorescence increase could be observed with such probes, but also that imaging was possible in various in vivo systems. Our evaluations with $\mathrm{HCy} 5$ revealed that a high increase in fluorescence could indeed be obtained in cell-free assays generating $\mathrm{HO}^{\circ}$ and $\mathrm{O}_{2}{ }^{\circ-}$. However, very stringent experimental conditions were required and small variations in the protocol resulted in a 10-fold decrease of the turn-on response. A possible explanation for these observations could be that as with cyanines, hydrocyanines could form $\mathrm{H}$-aggregates in water and buffer, especially at higher concentrations, altering their sensing behavior. ${ }^{[216]}$ Oxidation of HCy5 was also followed in cell-based assays, where a clear fluorescence increase was observed. Physical stress induced on differentiated epithelial cell monolayers by scratch-wounding showed that the cells at the border of the injury produced ROS. Stress response mimicking was also achieved by addition of stimulants to non-differentiated Caco-2 cells with subsequent monitoring of the turn-on response of the sensor. $\mathrm{HCy} 5$ successfully sensed the oxidative stress induction and its detection 
capacity was found to be similar to that of other fluorophores frequently used in ROS sensing (DHR and DCFHDA). To estimate the potential of the sensor, the respiratory burst of neutrophils was chosen to imitate a relevant situation occurring in host defense, as explained in Chapter 1. After isolating human neutrophils from fresh blood, the respiratory burst was chemically induced with PMA and subsequently detected with $\mathrm{HCy} 5$ as well as the control dyes DHR and DCFHDA. ROS production was observed with all three dyes and their turn-on responses were found to be comparable, further supporting our findings in Caco- 2 cells.

Encouraged by these results, the cyanine dye was modified with a hydrophilic macromolecule to transform it into an extracellular sensor. PEGylation or modification with PGA reduced the internalization by Caco- 2 cells compared to the free dye. Unfortunately, a concomitant decrease in sensing capacity was observed.

A major obstacle in fluorescence spectroscopy in vitro and in vivo arises from signal fluctuation due to sample heterogeneity and concentration differences. This issue has been frequently addressed by implementing a second fluorescence emission in the probe, and also our extracellular probe design was adapted to take advantage of an additional fluorescence signal.

In Chapter 4, we linked the intracellular ROS-sensor HCy5 to a reporter fluorophore that provided a second fluorescence emission. An advantage of using hydrocyanines as ROS indicator was the possibility to modify the cyanine dye and introduce the ROS-sensing capacity as a last step in the synthesis by reducing cyanine to hydrocyanine. However, this synthetic strategy introduced challenges such as guaranteeing the stability of all building blocks towards the reducing conditions. Indeed, selecting a reporter dye with high stability towards $\mathrm{NaBH}_{4}$ proved to be crucial. The BODIPY-analogue Chromis500 was the most resistant fluorophore among the tested dyes, and was used as model reporter compound. By linking the two hydrophobic dyes Chromis500 and Cy5 with a well-defined, hydrophilic peptide-PEG spacer, the ratiometric probe was rendered predominantly extracellular. The spacer was improved in Chapter 5 by introducing a modular unit consisting of complementary PNA strands, which enabled the separate handling of the dyes (e.g. cyanine reduction) and a simple combination of the fluorophores as a last step. Also in this case, synthesis led to a well-defined bio-polymeric ratiometric ROS sensor. This modular approach offers an optimal starting point to build up a library with different sensors and reporter dyes that could be combined on-demand for specific experiments. 
Given their macromolecular size and hydrophilic nature, the ratiometric probes were not internalized by differentiated Caco- 2 monolayers and in both cases, functional extracellular ROS-sensors were obtained. The detection specificity of the ratiometric probes shifted towards $\mathrm{O}_{2}{ }^{--}$in cell-free assays, thereby creating a specific probe for $\mathrm{O}_{2}{ }^{-*}$. The modular sensor was also tested in tissue culture and a doubling in ratiometric signal was observed. A summary of our main results is provided in Table 6-1.

Table 6-1: Summary of the main results of this Ph.D. thesis.

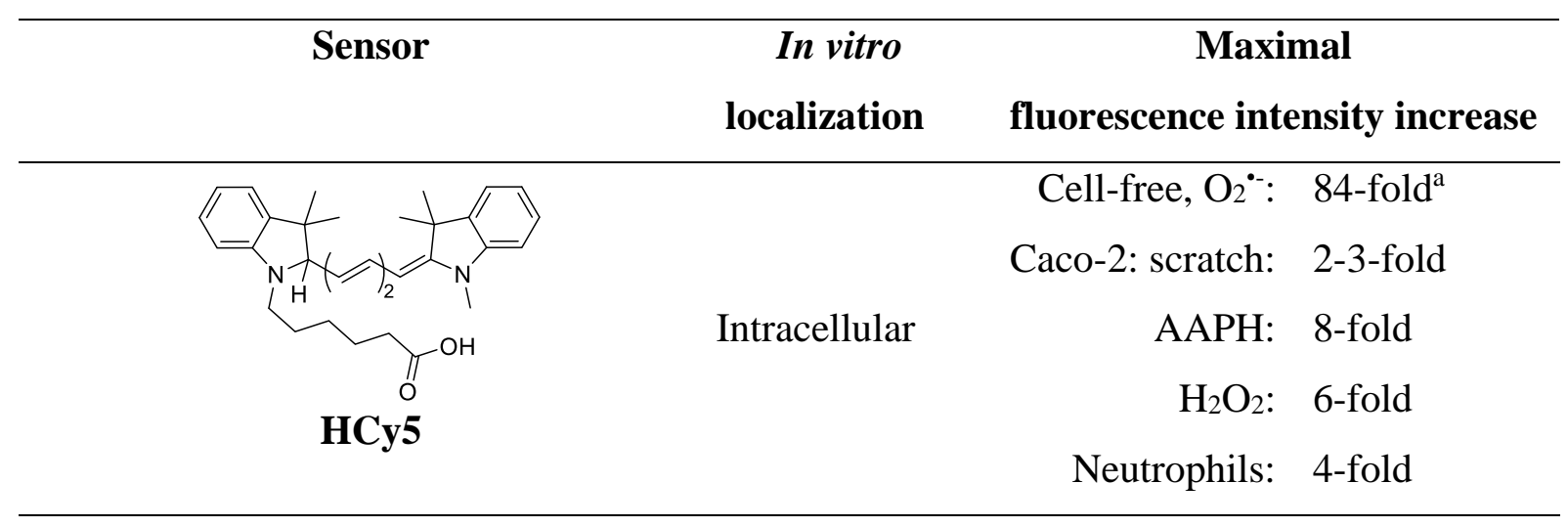

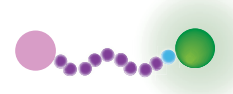

HCy5-(PEG 10)6-PGA-Chromis

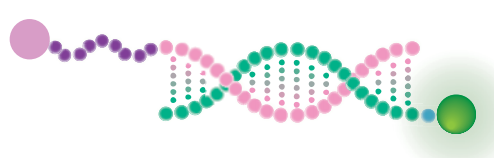

HCy5-(PEG 10$)_{3}$-PNA:PNA-Chromis
Extracellular $\quad \mathrm{O}_{2}{ }^{\circ-}$, cell-free: 2.3 -fold $^{\mathrm{b}}$

${ }^{\mathrm{a}}:\left[\mathrm{O}_{2}{ }^{\circ}\right]=20 \mu \mathrm{M},{ }^{\mathrm{b}}:\left[\mathrm{O}_{2}{ }^{\circ-}\right]=100 \mu \mathrm{M}$

Despite the interesting results obtained with our modular prototype for sensing extracellular $\mathrm{O}_{2}{ }^{-*}$, derivatization of $\mathrm{HCy} 5$ seemed to decrease the switch-on ability of the HCy5 in cell-free assays. ${ }^{[40,180]}$ Indeed, as also reported by two other groups who modified hydrocyanines for imaging purposes (derivatizing $\mathrm{HCy} 5$ with $\mathrm{PEG}^{[190]}$ or coupling them to a nano-carrier ${ }^{[191]}$ ), the detected response towards $\mathrm{O}_{2}{ }^{-}$and $\mathrm{HO}^{*}$ was lower compared to the original report. ${ }^{[190,191]}$ Taken together, these findings suggest that coupling the (hydro)cyanines to a macromolecule may influence the fluorescence properties, either due to potential effects on the cis/trans isomerization of the polymethine chain and/or the sensing ability, accounting for the reduced 
effect. ${ }^{[181]}$ Nevertheless, it is worth noting that in the cell assays the increase in the fluorescence signal obtained with the ratiometric probe was in the same order of magnitude than that reported with the commercially available HCy5 (ROSStarTM 650). ${ }^{[215]}$ This would indicate that the switch-on response of unconjugated $\mathrm{HCy} 5$ is highly influenced by experimental settings and needs carefully controlled conditions to obtain reliable read-outs, an observation that we also made during our validation studies in Chapter 3.

a)

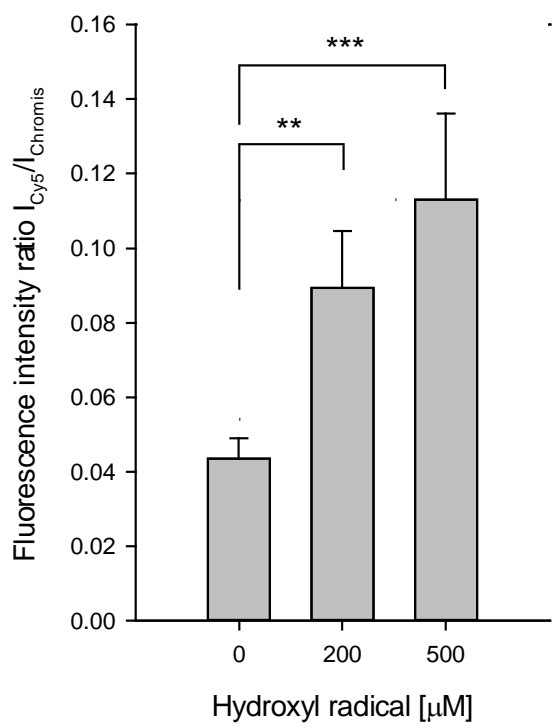

b)

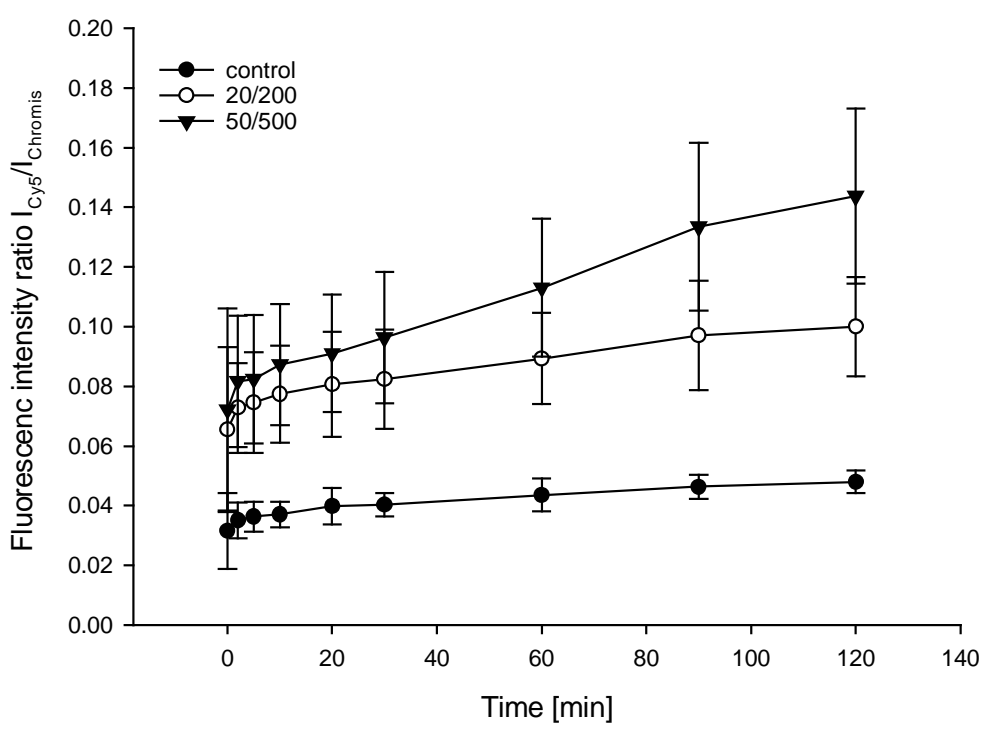

Figure 6-1: Sensing hydroxyl radicals of Chromis-PGA-( $\left.\mathrm{PEG}_{10}\right)_{6}-\mathrm{HCy} 5(10 \mu \mathrm{M})$ with the Fenton's reaction $\left(\left[\mathrm{FeSO}_{4}\right] /\left[\mathrm{H}_{2} \mathrm{O}_{2}\right]\right)$ in presence of $50 \mathrm{mg} / \mathrm{mL} \mathrm{BSA}$. Legend: $\left[\mathrm{FeSO}_{4}\right] /\left[\mathrm{H}_{2} \mathrm{O}_{2}\right]$ in $\mu \mathrm{M}$. control: 2 at $10 \mu \mathrm{M}$. (Mean $\pm \mathrm{SD}, \mathrm{n}=9$ ).

Even though the detection capacity of our ratiometric probes was reduced compared to HCy5, the detection of ROS in cell-based assays was possible. To more accurately evaluate the full potential of our sensors, in vivo studies would be a valuable addition. With the presented prototypes, however, the selected fluorophores pose a major limitation for in vivo applications as they do not emit in the NIR and especially Chromis should be replaced by a higher wavelength dye. Additionally, the limited sensitivity of our probes may also be an issue for their in vivo use. Nevertheless, a preliminary sensing experiment was carried out in presence of BSA, a solubilizing agent abundantly present in blood. The $\mathrm{HO}^{*}$ detection capacity of ChromisPGA-(PEG10)6-HCy5 was tested in presence of BSA and to our surprise, an up to 3-fold increase in ratiometric signal was observed (Figure 6-1). This indicates that the turn-on behavior of hydrocyanines - coupled or not - is greatly dependent on the solubilizing power of the used medium, and also that the aggregation of the hydrophobic dye may reduce the sensing behavior. Recent findings from Chattaraj et al. corroborate these data, as they reported that even quinones 
can oxidize a hydrophobically modified hydrocyanine derivative if they are completely solubilized in the oil that is used as imaging medium. ${ }^{[217]}$

To summarize, after careful validation of HCy5 as ROS-sensor, an extracellular ratiometric ROS-sensing probe was developed. In addition, a novel platform for analyte sensing was introduced for the first time, using PNA-PGA-(PEG 10$)_{x}$ as combinatorial unit. Due to the PNA spacer, the building blocks may be chosen with high versatility, as the fluorophores are combined with a simple hybridization step. Specific $\mathrm{O}_{2}{ }^{-\bullet}$ chemodosing revealed that detection of oxidative stress in cell-free assays as well as cell culture is possible and is comparable for the modular ratiometric imaging probe 7 and the covalently linked sensor 5. Although the sensitivity towards $\mathrm{O}_{2}{ }^{--}$of the proposed fluorophores could be further improved, the concept of a general modular platform for extracellular ratiometric sensing shows potential and could be expanded to other analytes. ${ }^{[34]}$ 


\section{Appendix}

\section{Characterization of molecules in Chapter 3}

\section{Cyanine5 (Cy5)}

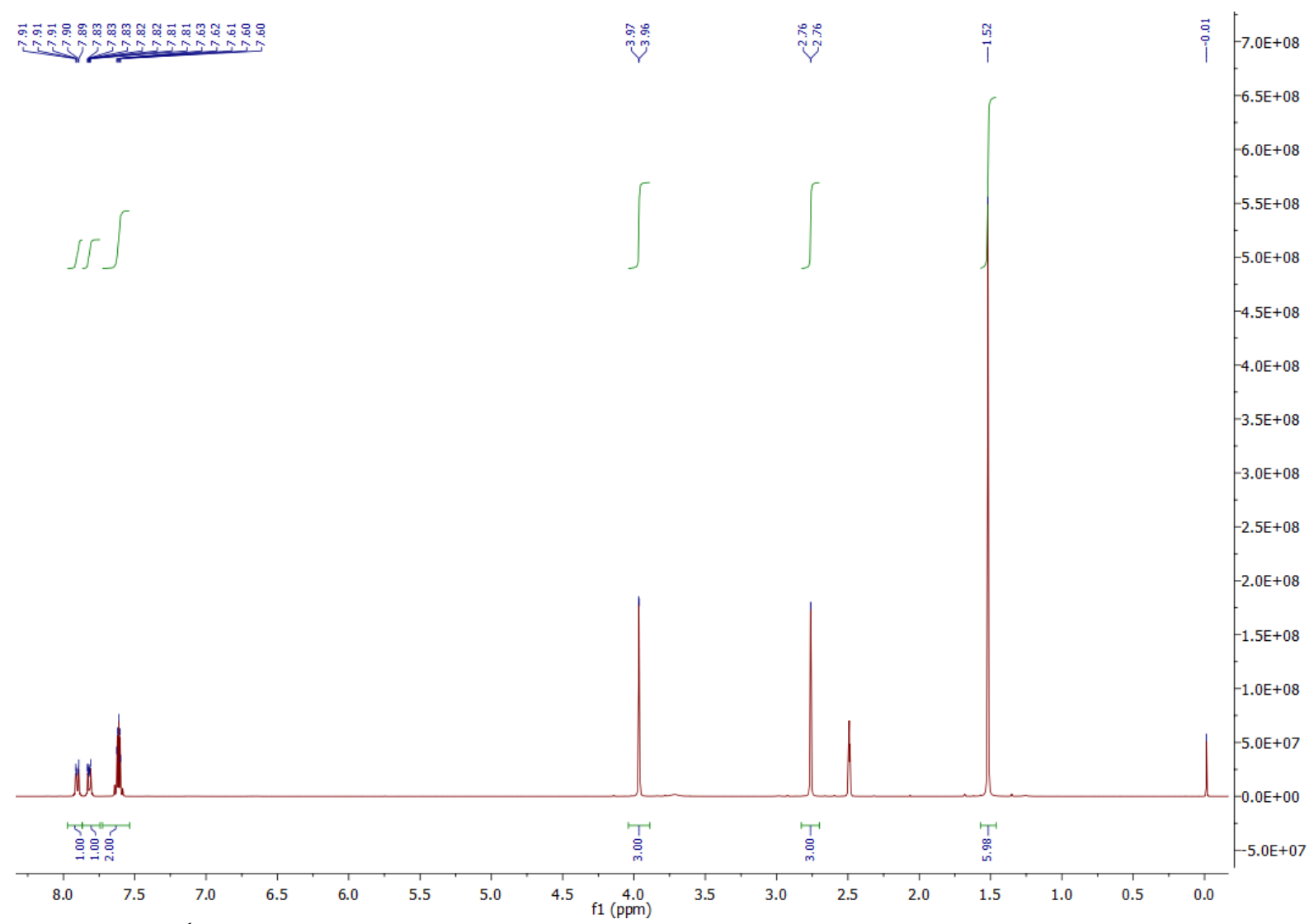

Figure A1: ${ }^{1} \mathrm{H}-\mathrm{NMR}$ spectrum of 1,2,3,3-tetramethyl-3H-indol-1-ium iodide $\mathbf{1}$. 


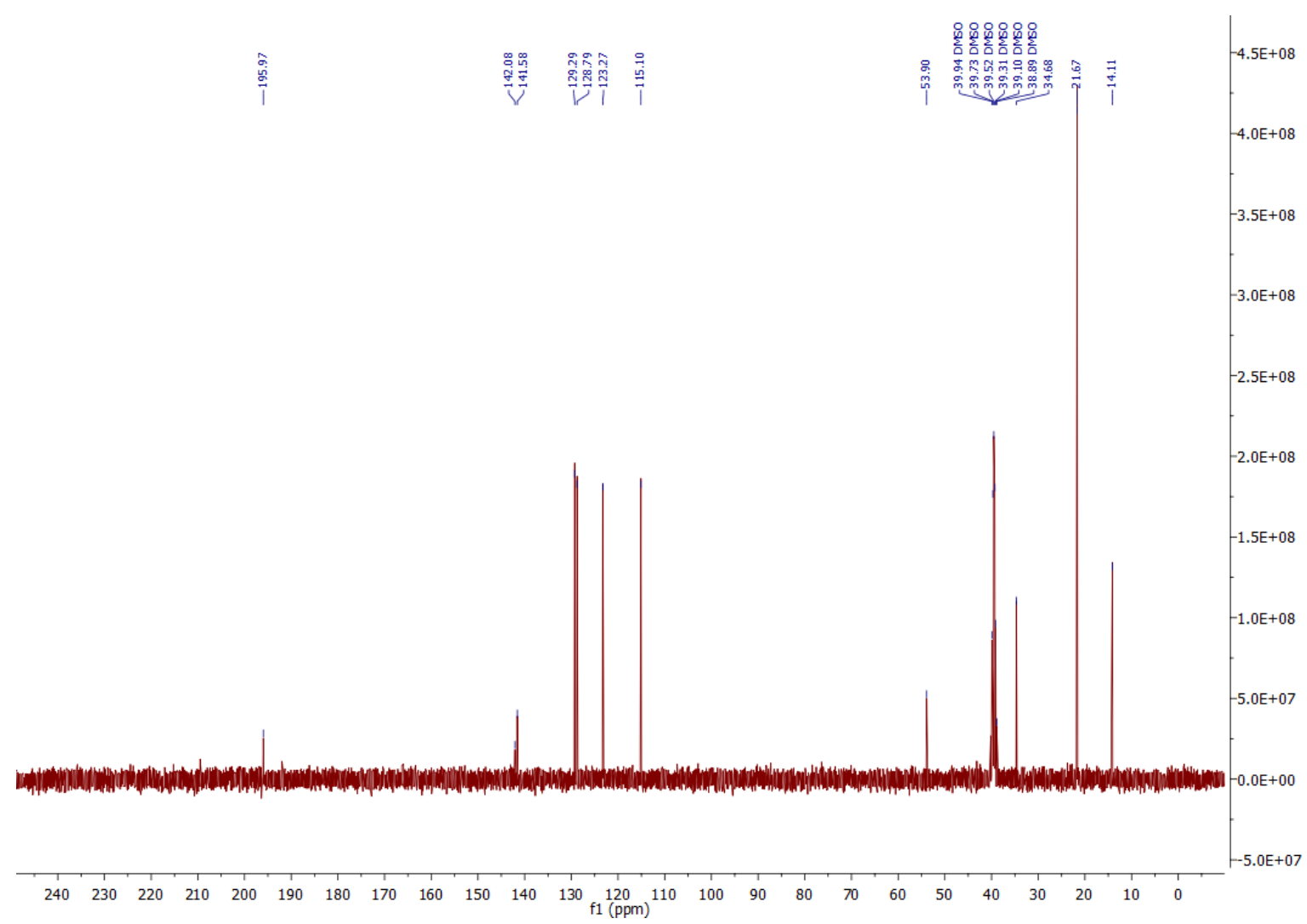

Figure A2: ${ }^{13} \mathrm{C}-\mathrm{NMR}$ spectrum of 1,2,3,3-tetramethyl-3H-indol-1-ium iodide $\mathbf{1}$.

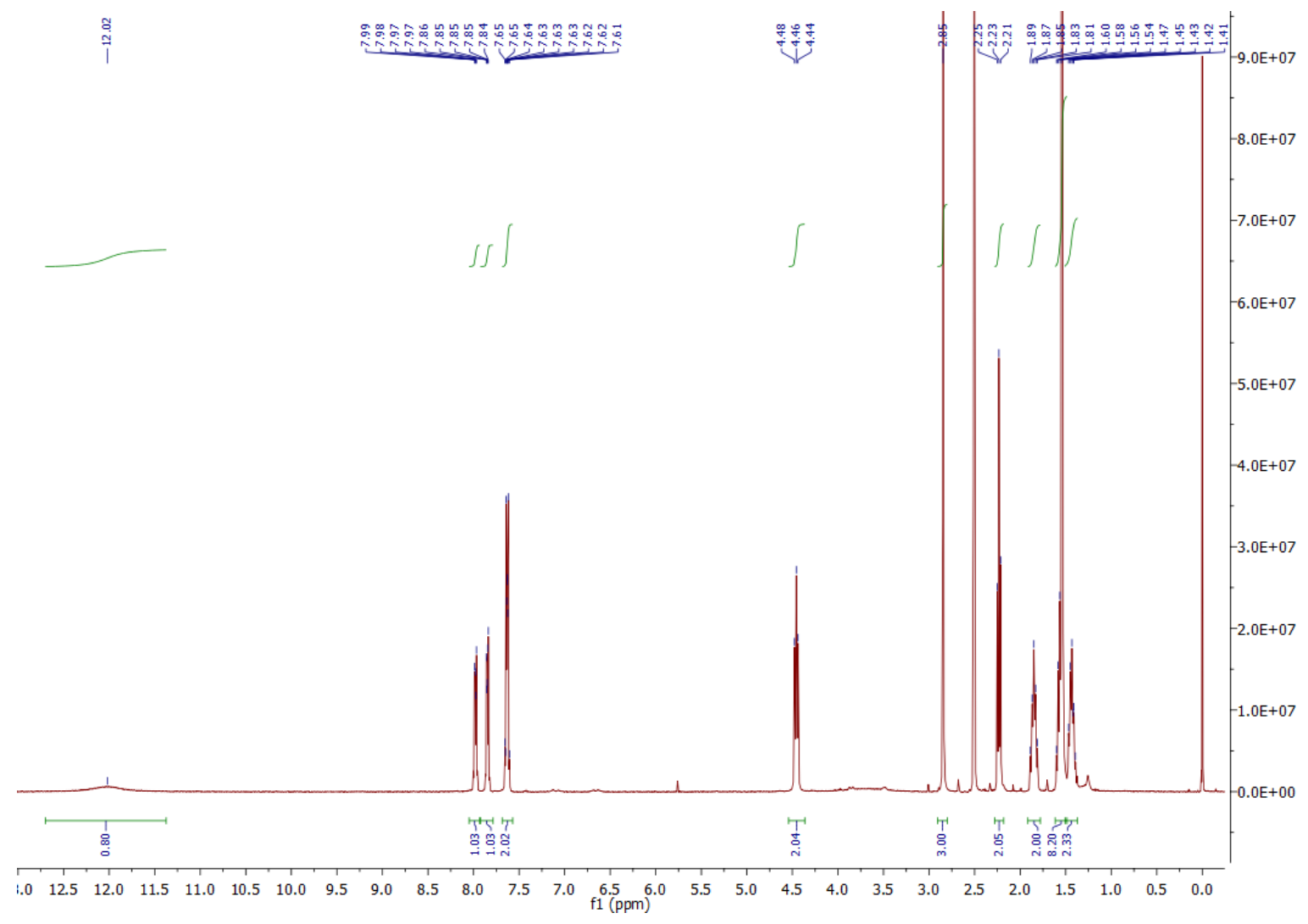

Figure A3: ${ }^{1} \mathrm{H}-\mathrm{NMR}$ spectrum of 1-(5-carboxypentyl)-2,3,3-trimethyl-3H-indol-1-ium iodide 2. 


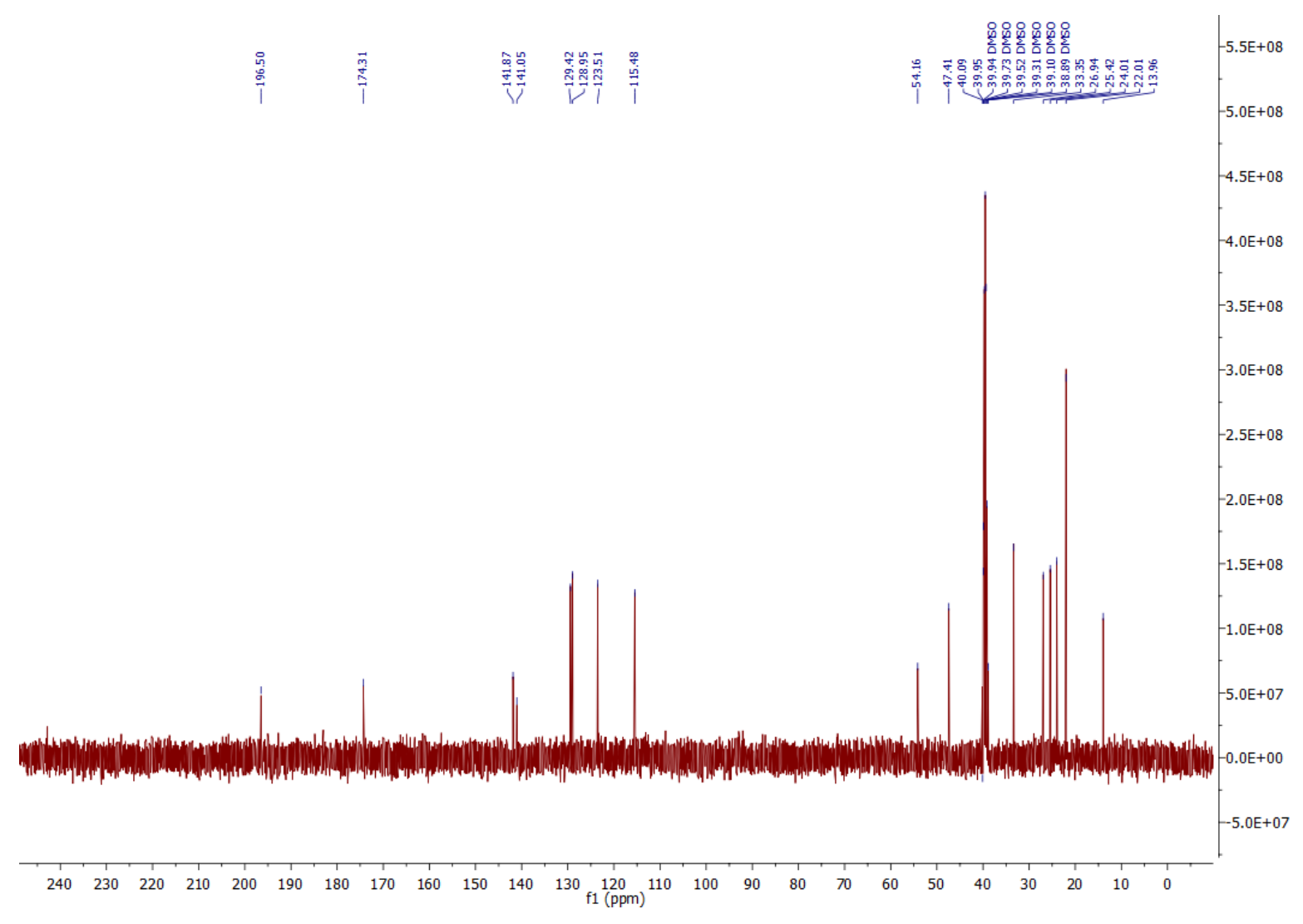

Figure A4: ${ }^{13} \mathrm{C}$-NMR spectrum of 1-(5-carboxypentyl)-2,3,3-trimethyl-3H-indol-1-ium iodide 2.

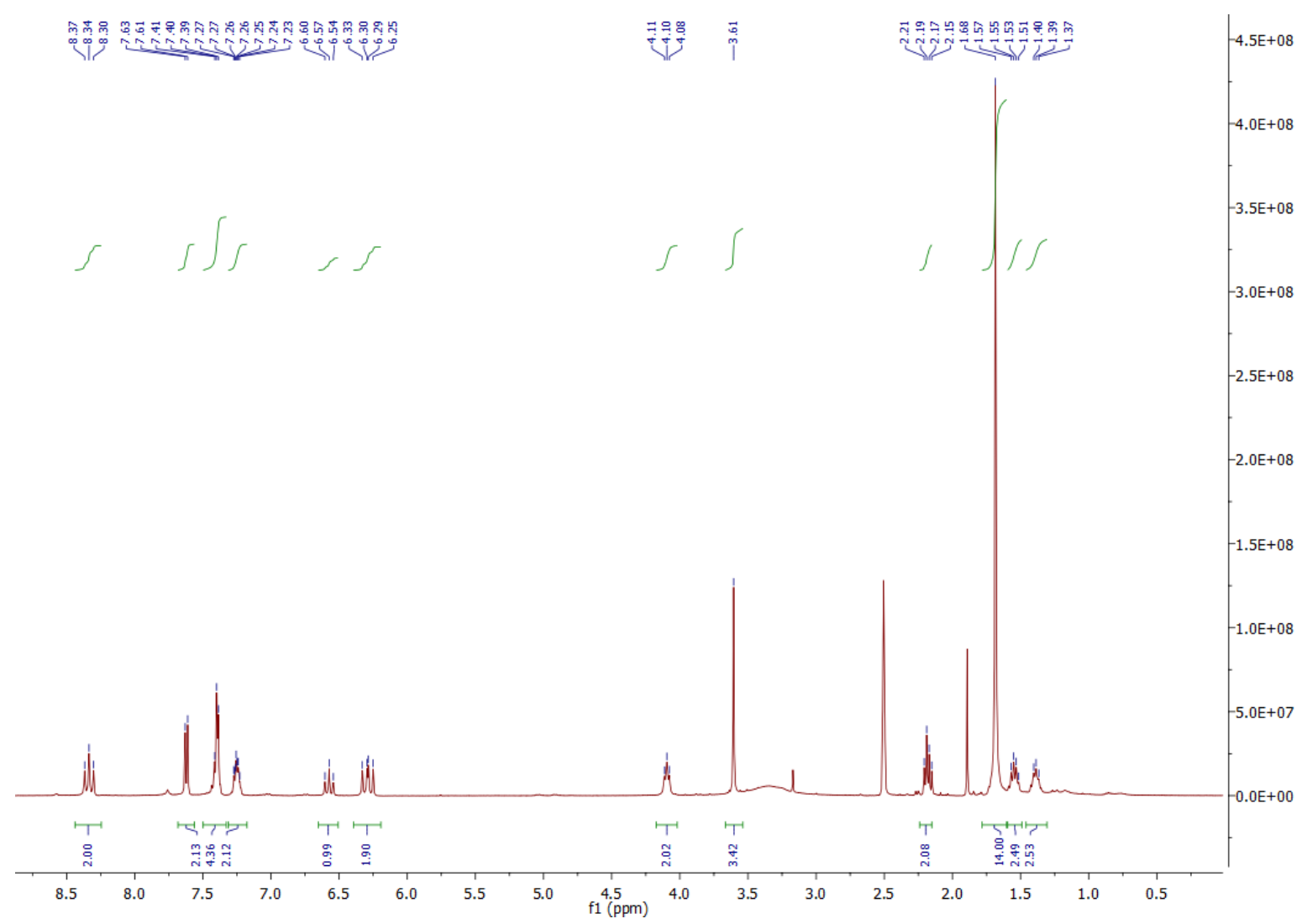

Figure A5: ${ }^{1} \mathrm{H}-\mathrm{NMR}$ spectrum of Cy5 3. 


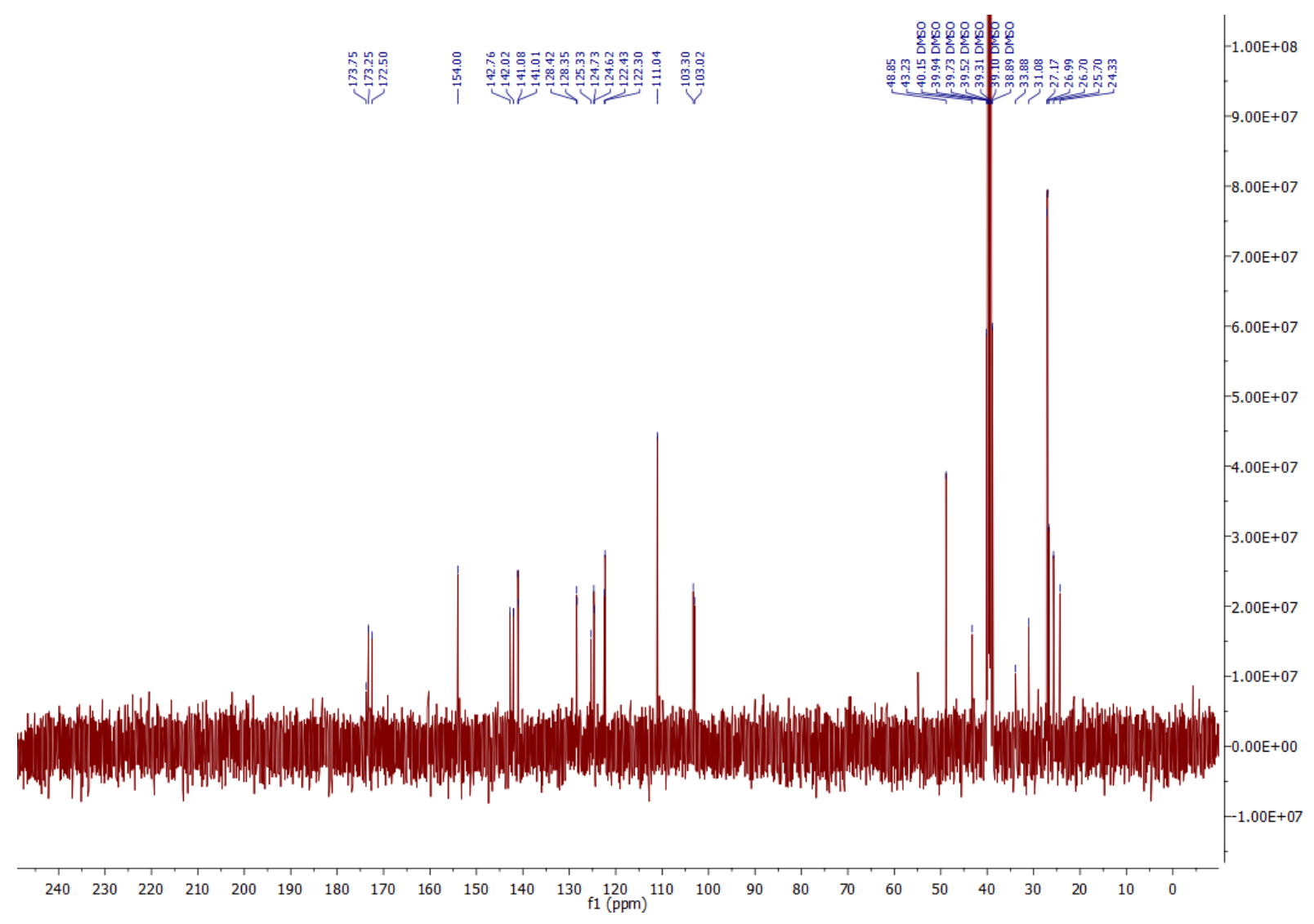

Figure A6: ${ }^{13} \mathrm{C}-\mathrm{NMR}$ spectrum of Cy5 3.

\section{Cy5-PEG}
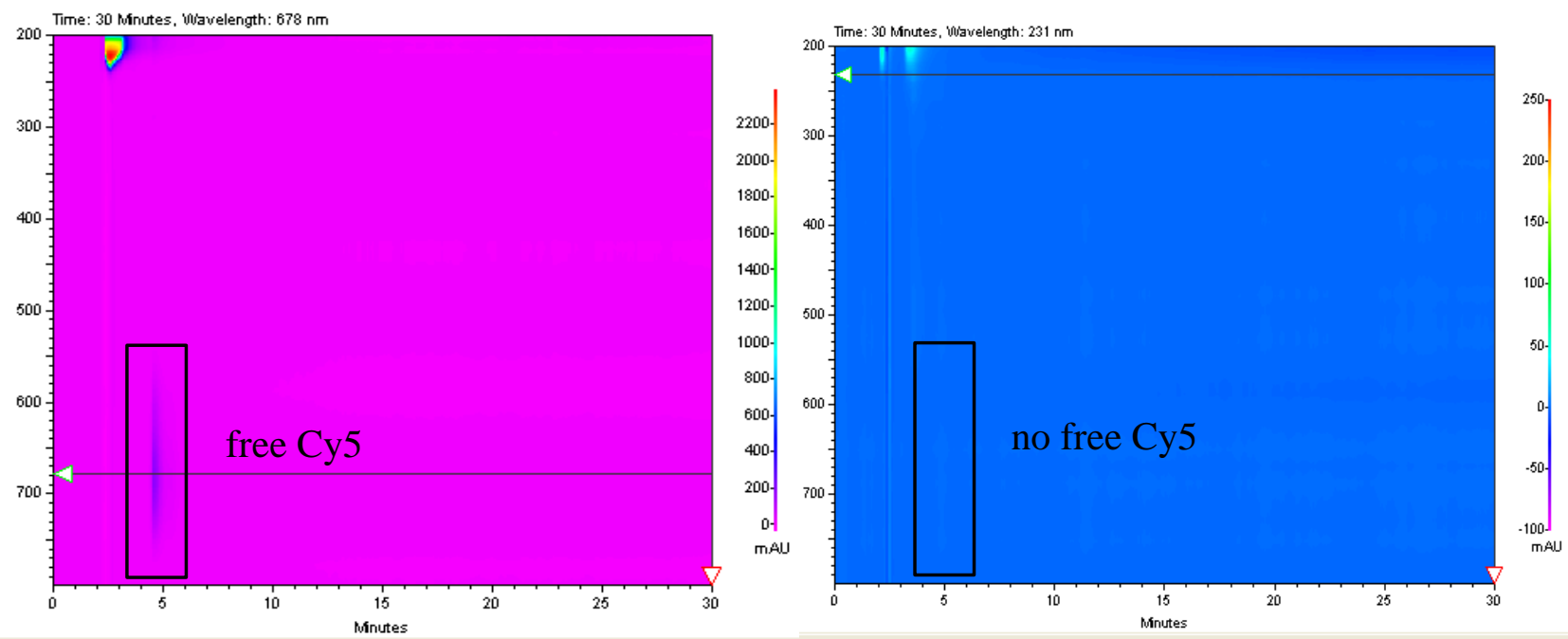

Figure A7: HPLC analysis of Cy5 (left) and Cy5-PEG (right). The absence of a peak at $4.8 \mathrm{~min}$ corresponding absorbing at $650 \mathrm{~nm}$ was detected to verify successfully size-exclusion chromatography with Sephadex ${ }^{\mathrm{TM}} \mathrm{G} 25$. 


\section{Cy5-PGA}
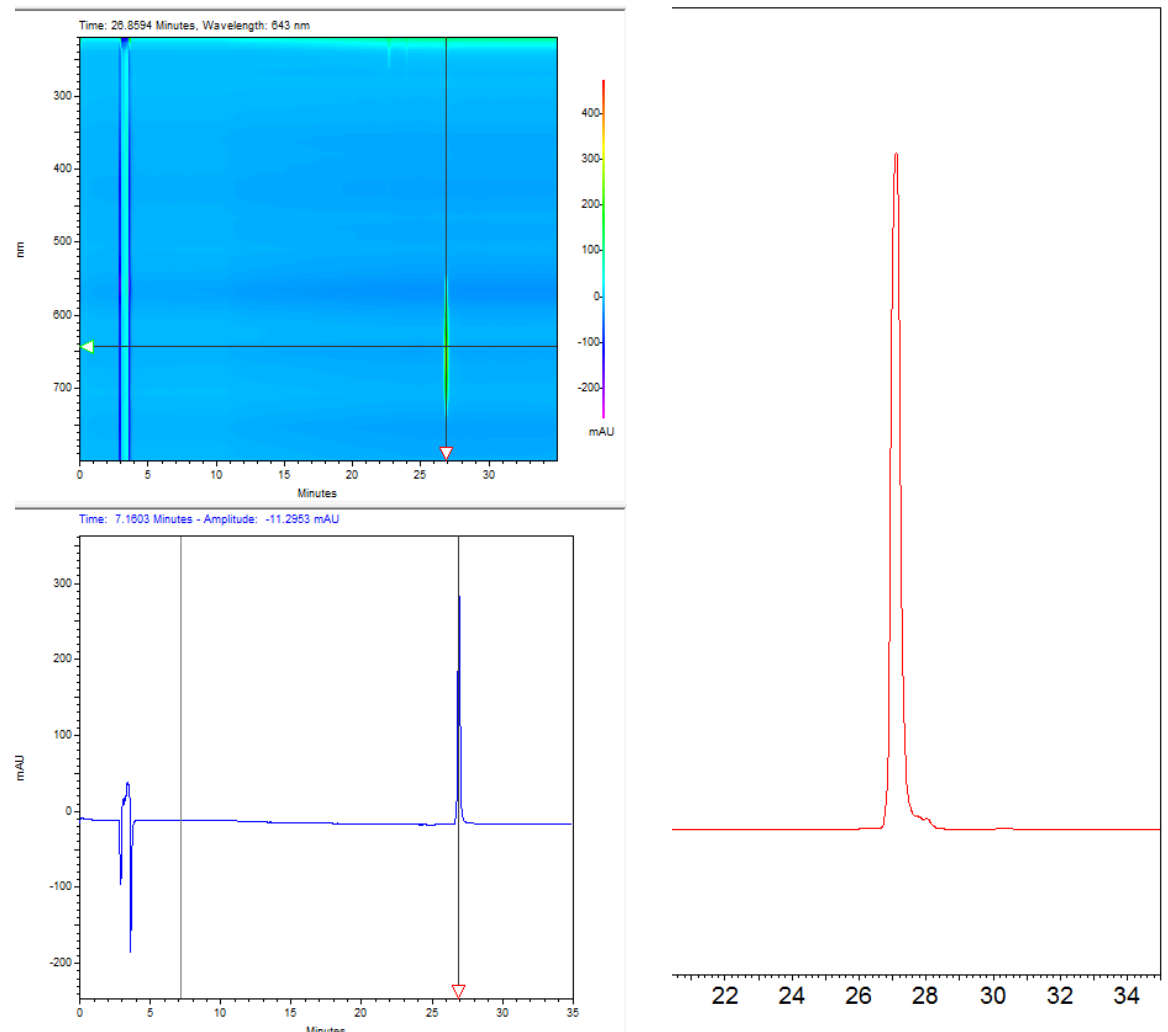

Figure A8: HPLC analysis of Cy5-PGA: Absorbance chromatogram (left) and fluorescence chromatogram (right, $\lambda_{\mathrm{ex}}=620 \mathrm{~nm}, \lambda_{\mathrm{em}}=665 \mathrm{~nm}$ ).

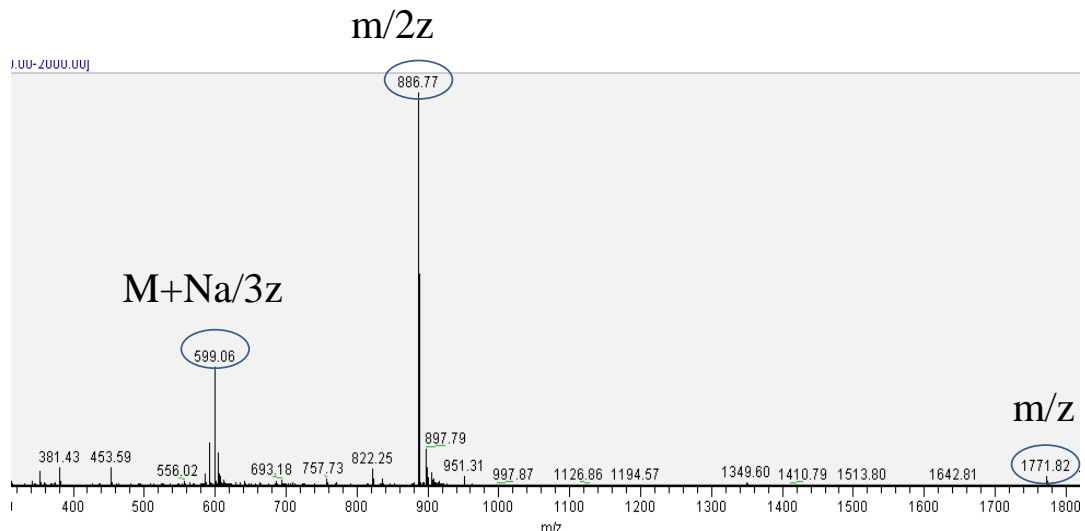

Figure A9: MS analysis of Cy5-PGA. 


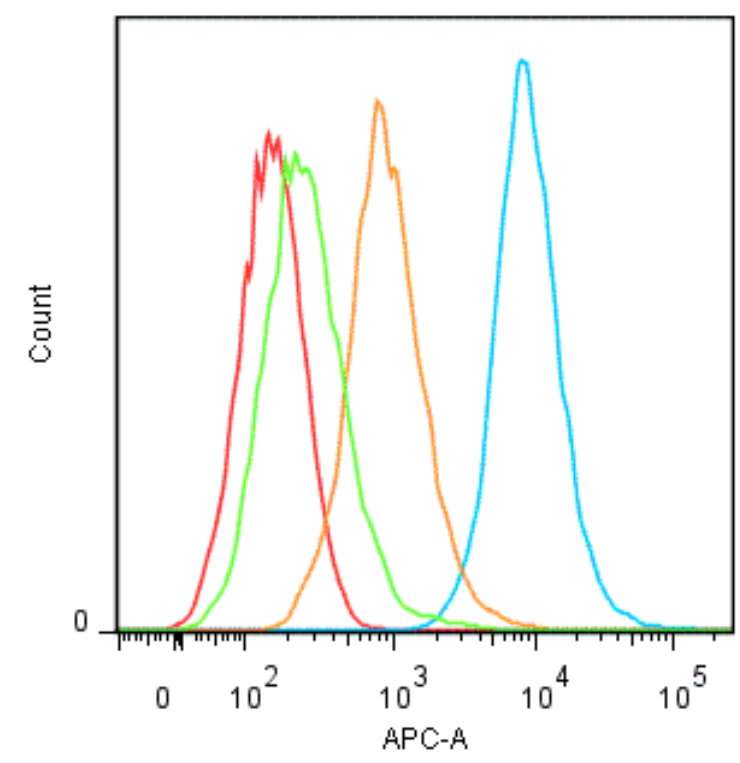

\begin{tabular}{|l|l|}
\hline & \multicolumn{2}{|c|}{ TUBE NAME } \\
\hline \hline & PGA 1 \\
\hline \hline & PEG 1 \\
\hline \hline & CV5 1 \\
\hline \hline & control1 \\
\hline
\end{tabular}

Figure A10: Representative histogram for the uptake of Cy5-PGA, Cy5-PEG and Cy5 in differentiated Caco-2 monolayers. 


\section{Characterization of molecules in Chapter 4}

\section{Lys-PGA-(PEG 10$)_{x}-\mathrm{Cy} 5$}

Table A1: Expected m/z of the Lys-PGA-(PEG10)0-6-Cy5.

\begin{tabular}{|c|c|}
\hline Lys-PGA-(PEG 10$)_{x}-\mathrm{Cy} 5$ & MW [g/mol] \\
\hline Lys-PGA-(PEG 10$)_{0}-\mathrm{Cy} 5$ & 1960.55 \\
\hline Lys-PGA-(PEG 10$)_{1}-\mathrm{Cy} 5$ & 2471.66 \\
\hline Lys-PGA-(PEG 10$)_{2}-\mathrm{Cy} 5$ & 2983.27 \\
\hline Lys-PGA-(PEG 10$)_{3}-\mathrm{Cy} 5$ & 3494.88 \\
\hline Lys-PGA-(PEG 10$)_{4}-\mathrm{Cy} 5$ & 4006.49 \\
\hline Lys-PGA-(PEG 10$)_{5}-\mathrm{Cy} 5$ & 4518.10 \\
\hline Lys-PGA-(PEG 10$)_{6}-\mathrm{Cy} 5$ & 5029.71 \\
\hline
\end{tabular}

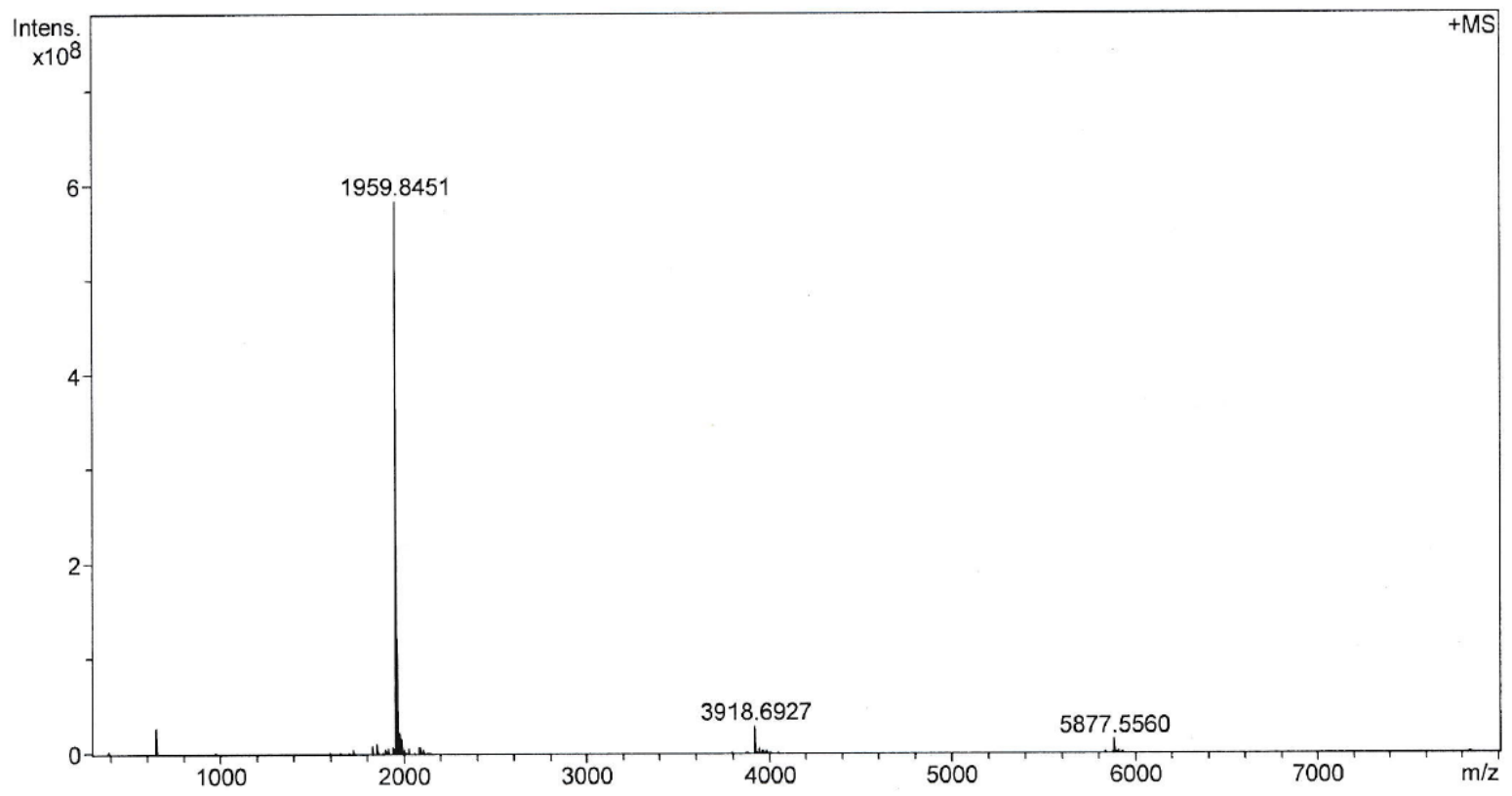

Figure A11: HRMS-MALDI of Lys-Glu $10-\left(\mathrm{PEG}_{10}\right)_{0}-\mathrm{Cy} 5$. 


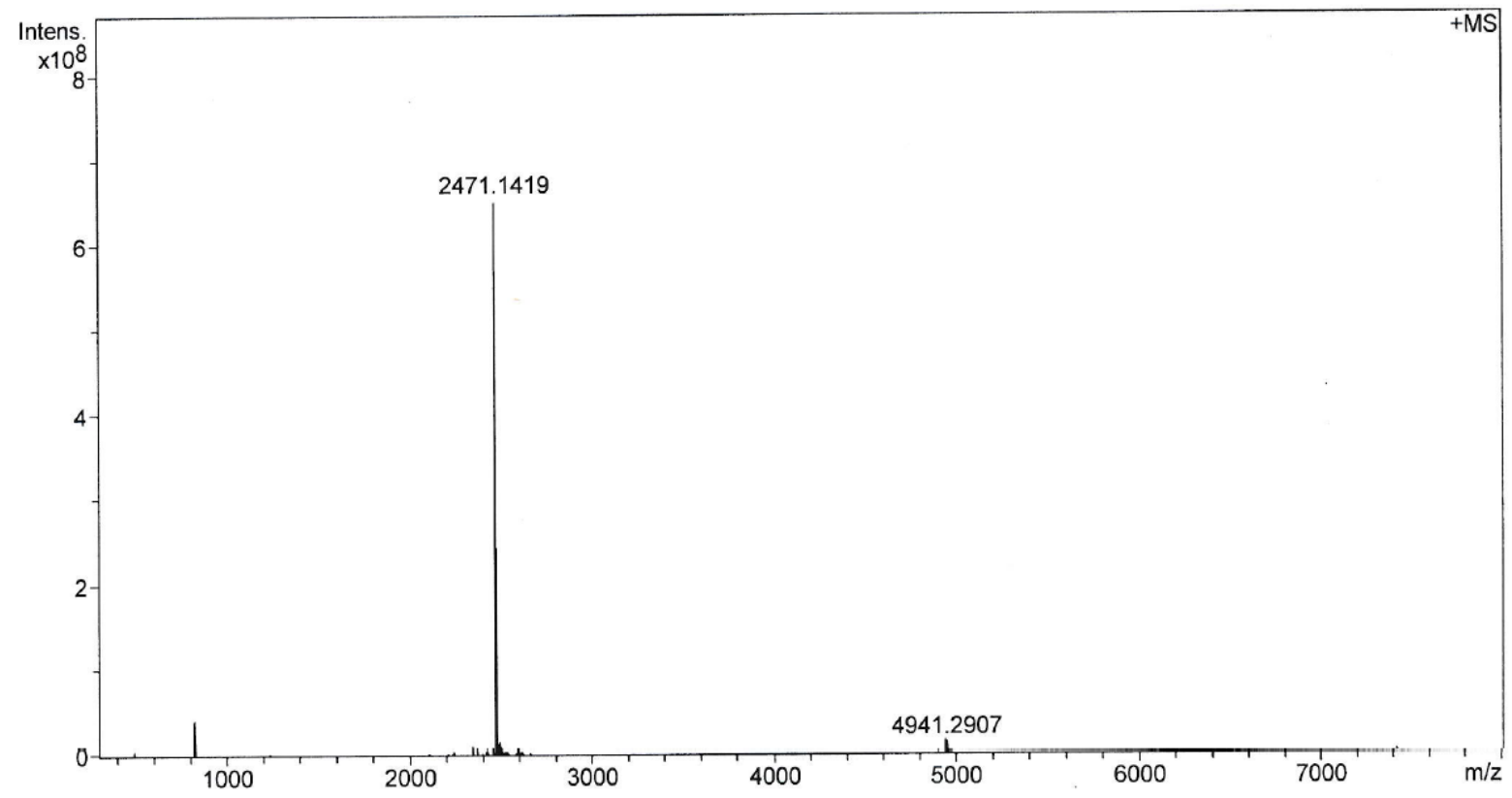

Figure A12: HRMS-MALDI of Lys-Glu $10-\left(\mathrm{PEG}_{10}\right)_{1}-\mathrm{Cy} 5$.

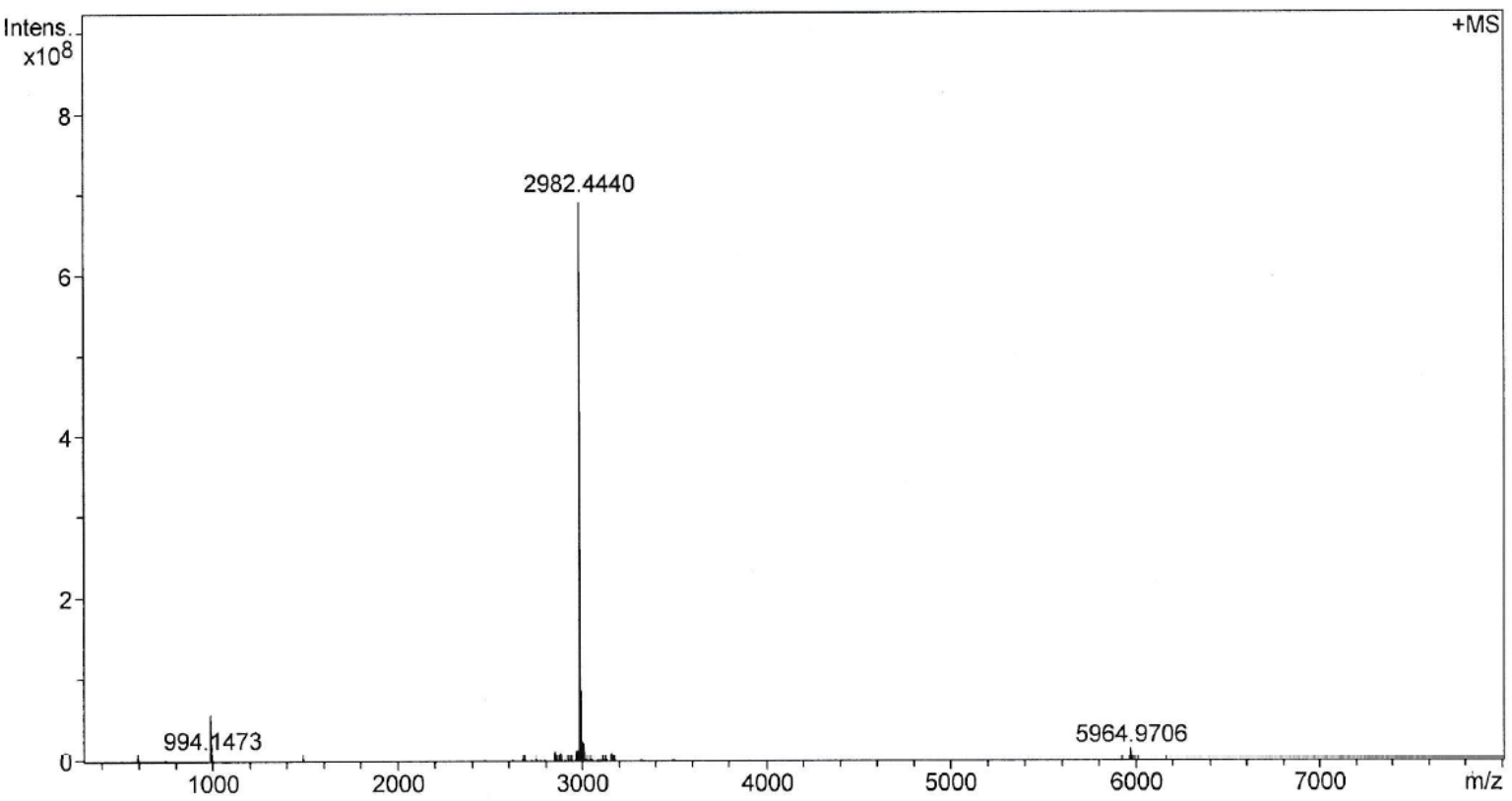

Figure A13: HRMS-MALDI of Lys-Glu10-(PEG 10$)_{2}-\mathrm{Cy} 5$. 


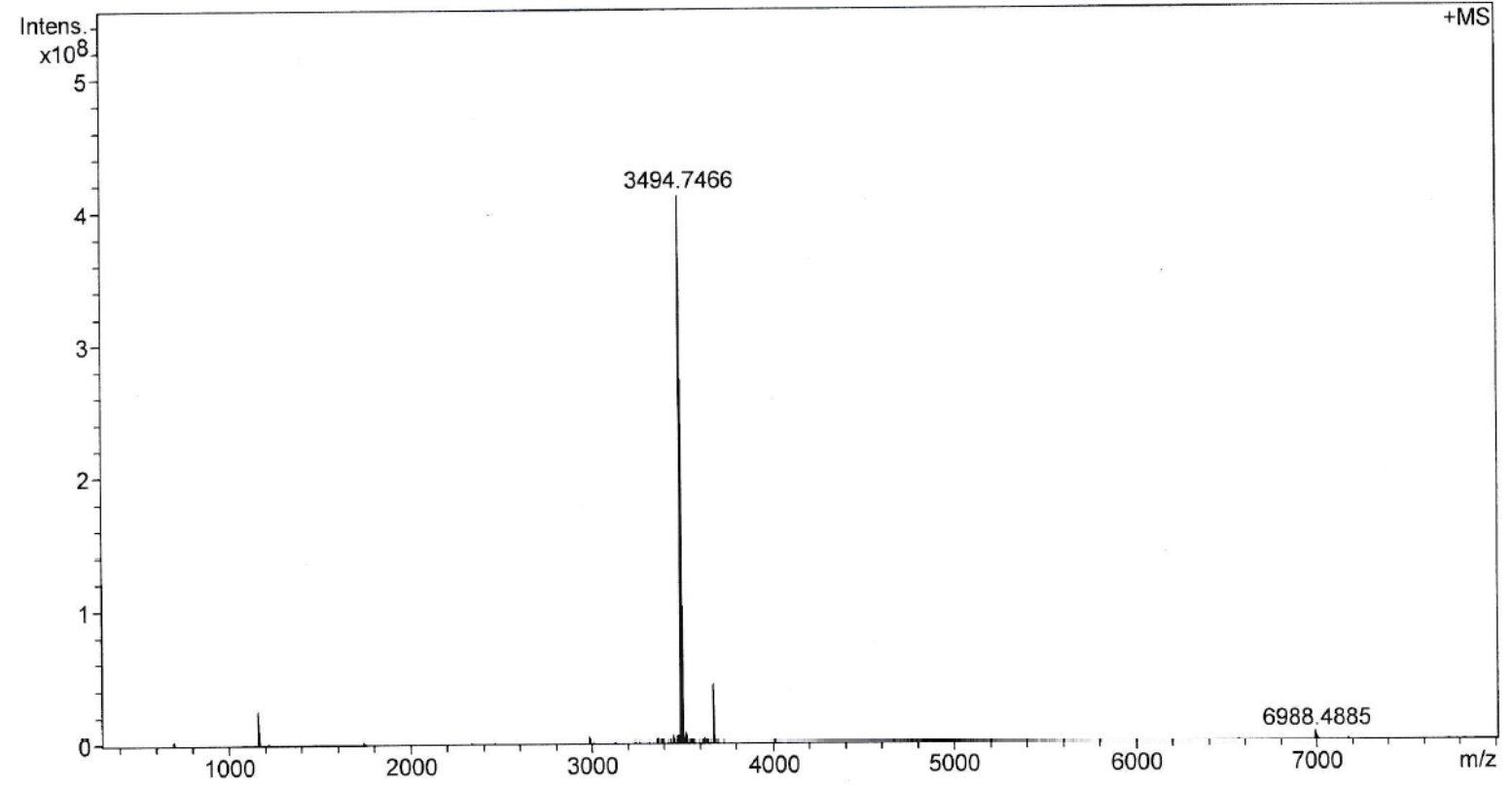

Figure A14: HRMS-MALDI of Lys-Glu10-(PEG10)3-Cy5.

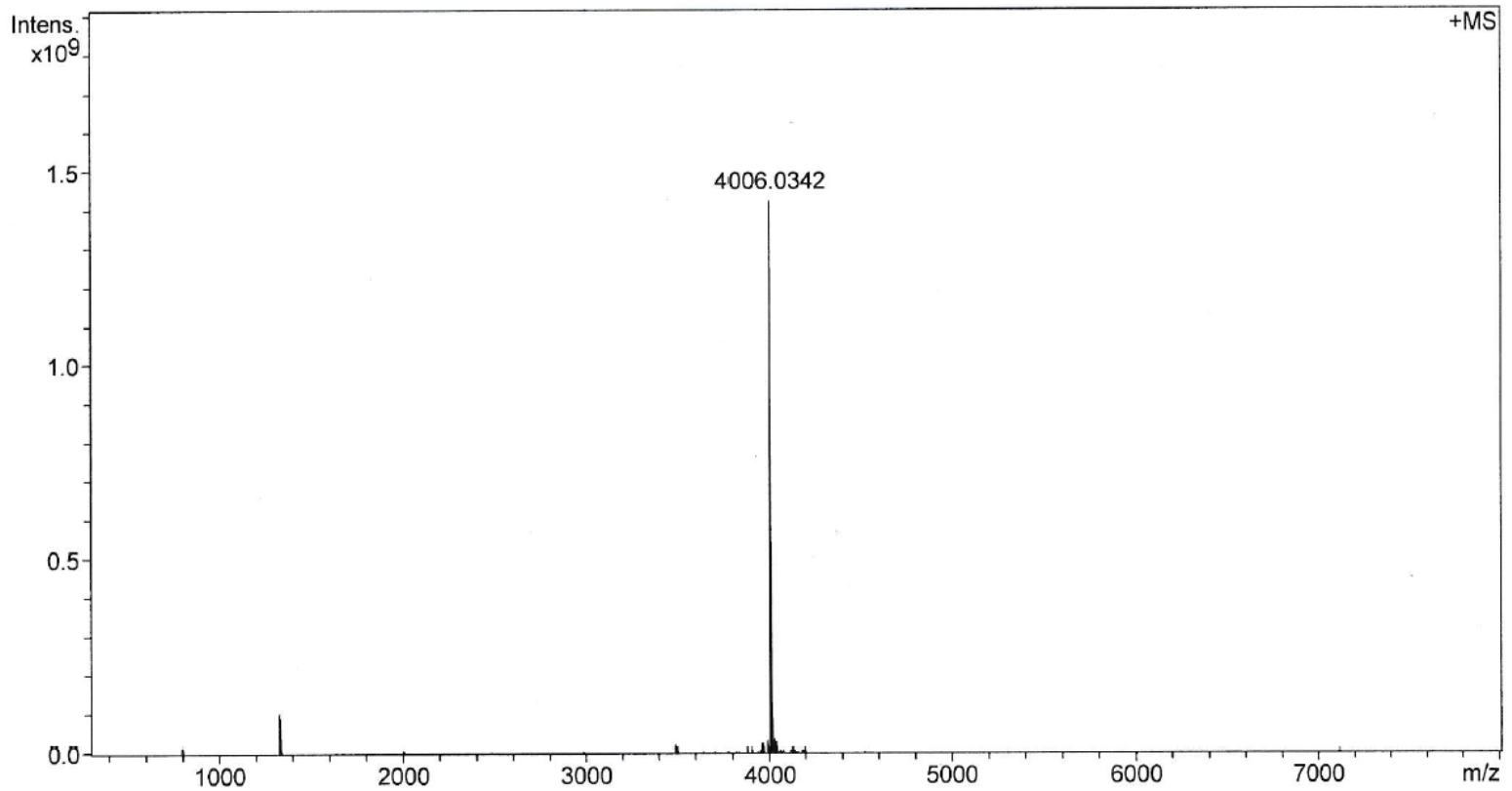

Figure A15: HRMS-MALDI of Lys-Glu10-(PEG10)4-Cy5. 


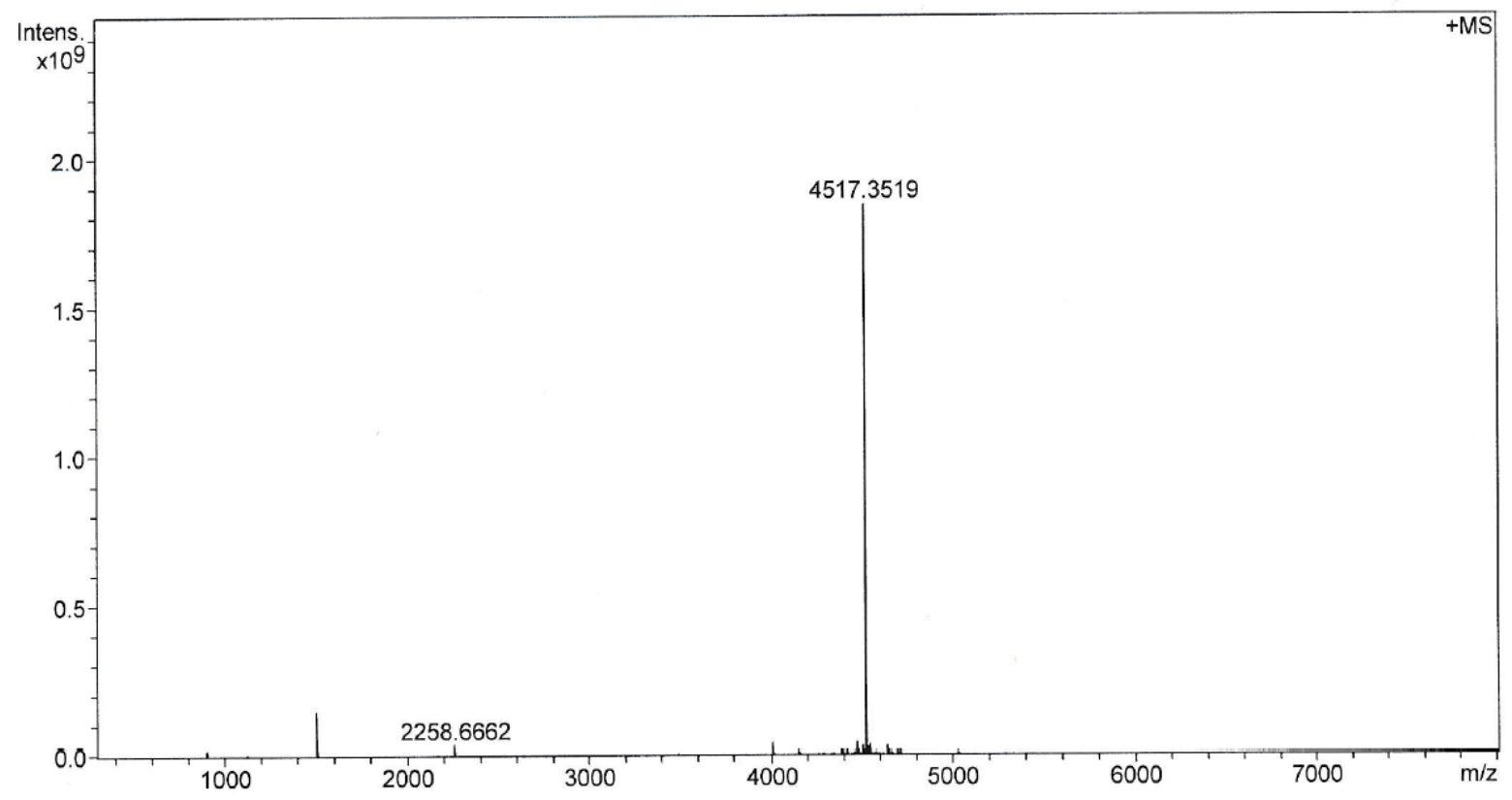

Figure A16: HRMS-MALDI of Lys-Glu10-(PEG10)5-Cy5.

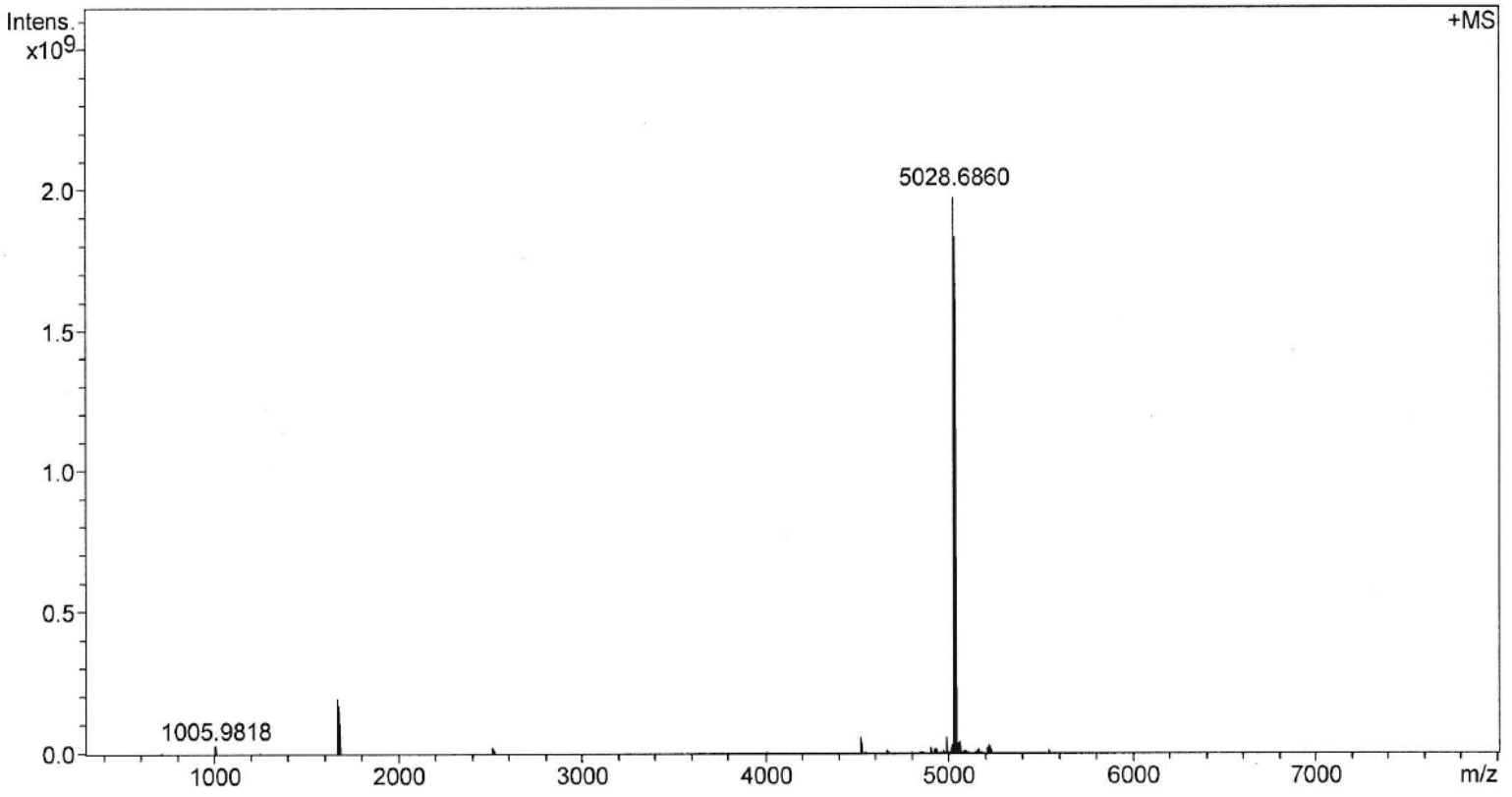

Figure A17: HRMS-MALDI of Lys-Glu 10-(PEG $\left.{ }_{10}\right)_{6}-\mathrm{Cy} 5$. 


\section{Chromis-PGA-(PEG 10$)_{x}-\mathrm{Cy} 5$}

Table A2: Expected m/z of the Chromis-PGA-(PEG10)0-6-Cy5.

\begin{tabular}{|c|c|}
\hline Chromis-PGA-(PEG 10$)_{x}-\mathrm{Cy} 5$ & $\mathrm{MW}[\mathrm{g} / \mathrm{mol}]$ \\
\hline Chromis-PGA-(PEG 10$)_{0}-\mathrm{Cy} 5$ & 2413.40 \\
\hline Chromis -PGA-(PEG 10$)_{1-C y 5}$ & 2925.01 \\
\hline Chromis -PGA-(PEG10)2-Cy5 & 3436.62 \\
\hline Chromis -PGA-(PEG 10$)_{3}-\mathrm{Cy} 5$ & 3948.22 \\
\hline Chromis -PGA-(PEG 10$)_{4}-\mathrm{Cy} 5$ & 4459.83 \\
\hline Chromis -PGA-(PEG10)5-Cy5 & 4971.44 \\
\hline Chromis -PGA-(PEG 10$)_{6}-\mathrm{Cy} 5$ & 5483.05 \\
\hline
\end{tabular}

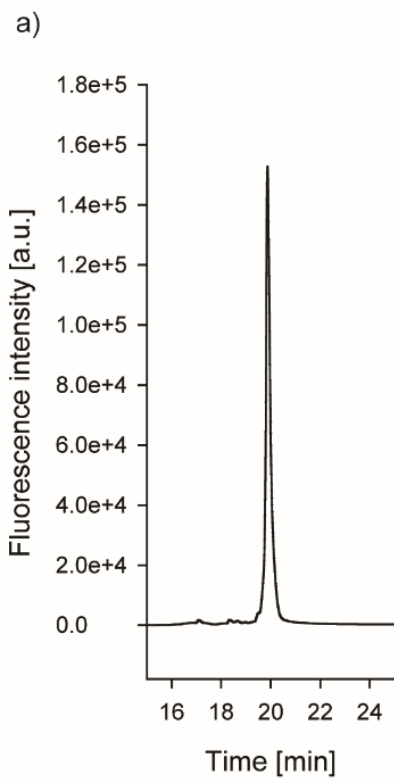

b)

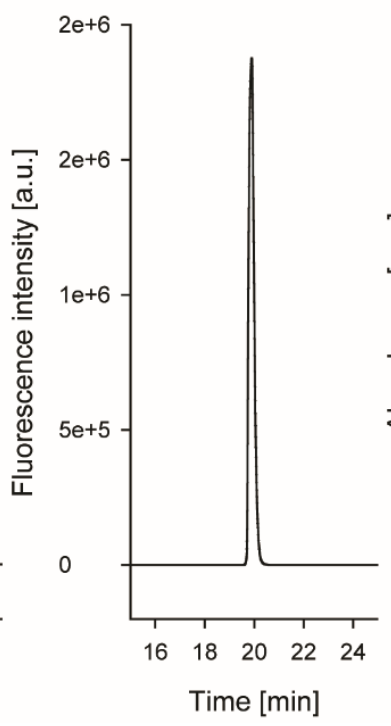

c)

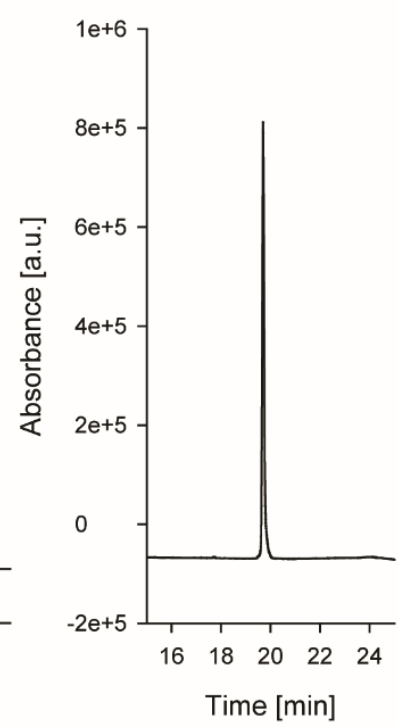

d)

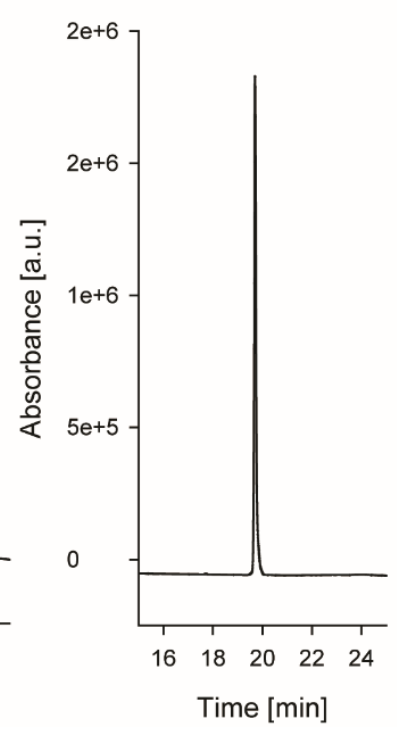

Figure A18: Verification of purity by HPLC analysis of Chromis-PGA-(PEG $\left.\mathrm{P}_{10}\right)_{0}-\mathrm{Cy} 5$. a) Fluorescence detection $\left.\left(\lambda_{\mathrm{ex}}=488 \mathrm{~nm}, \lambda_{\mathrm{em}}=525 \mathrm{~nm}\right) \mathrm{b}\right)$ fluorescence detection $\left(\lambda_{\mathrm{ex}}=620 \mathrm{~nm}\right.$, $\lambda_{\mathrm{em}}=665 \mathrm{~nm}$ ) c) absorbance at $500 \mathrm{~nm} \mathrm{~d}$ ) absorbance at $650 \mathrm{~nm}$. 


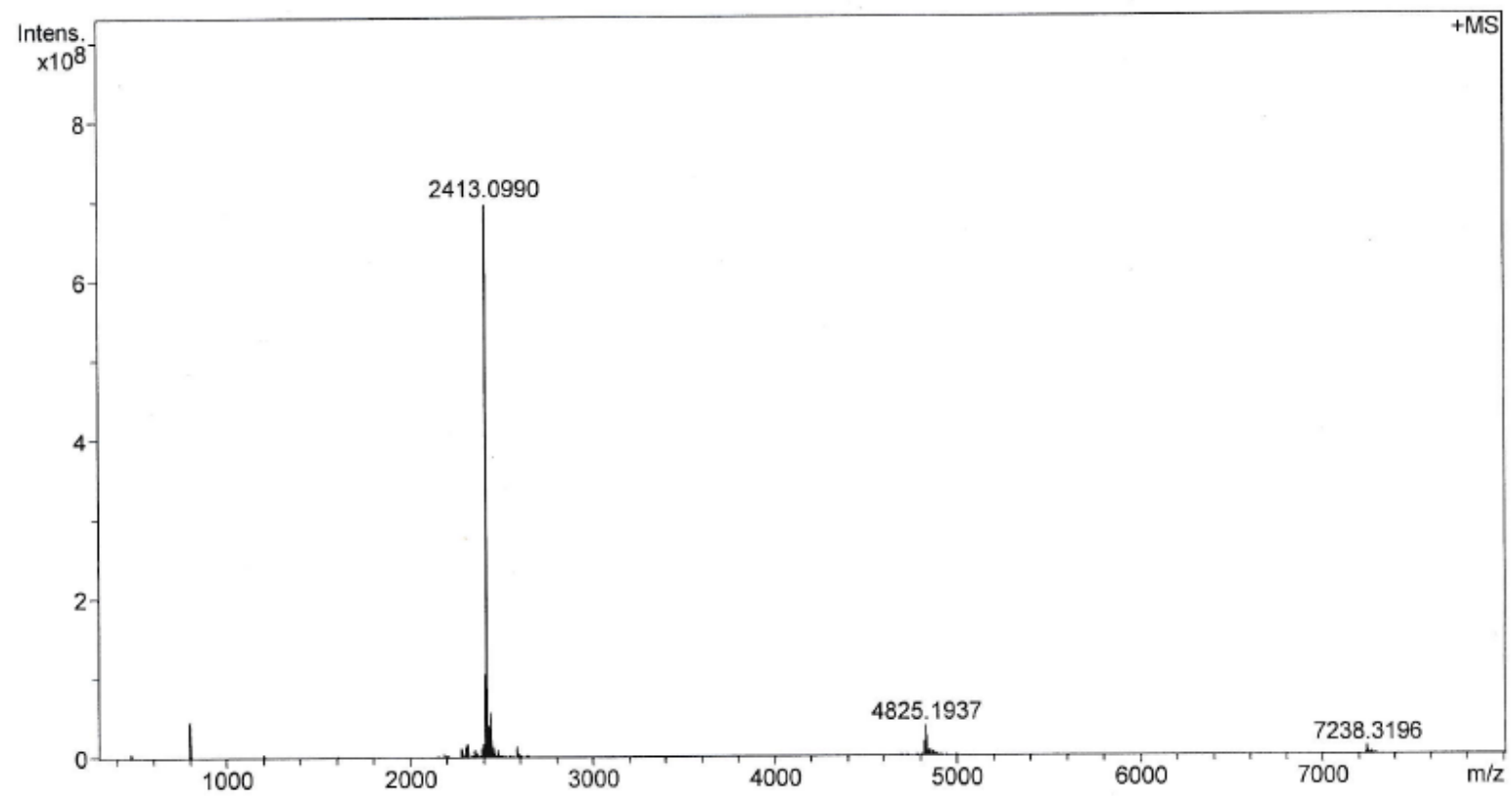

Figure A19: HRMS-MALDI of Chromis-PGA-(PEG $\left.{ }_{10}\right)_{0}-\mathrm{Cy} 5$.

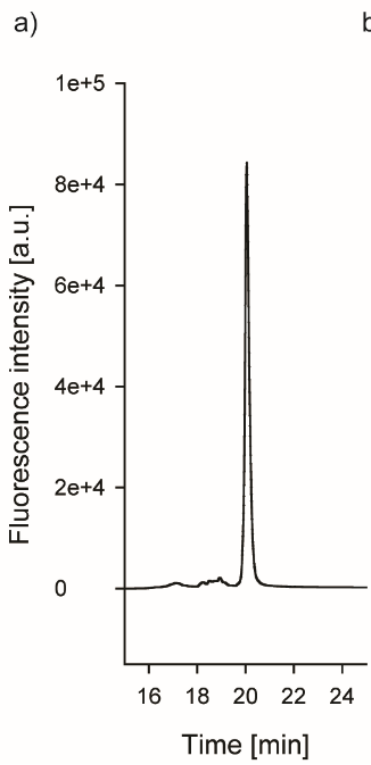

b)

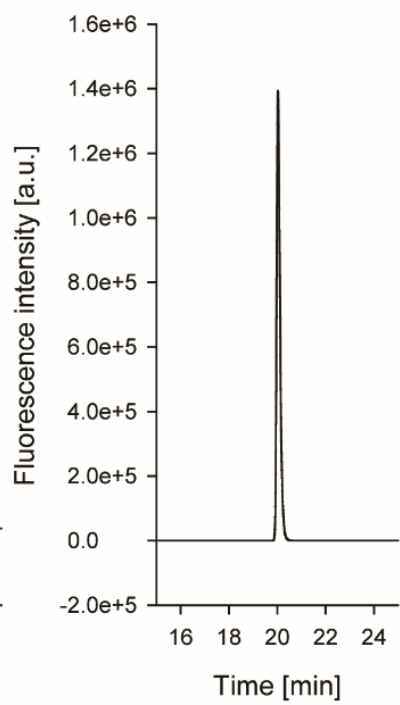

c)

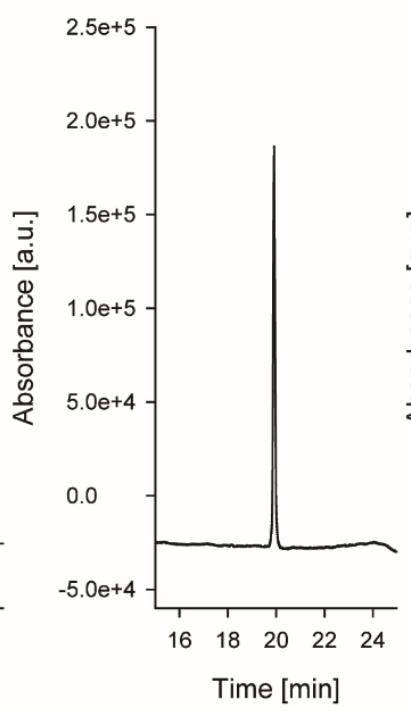

d)

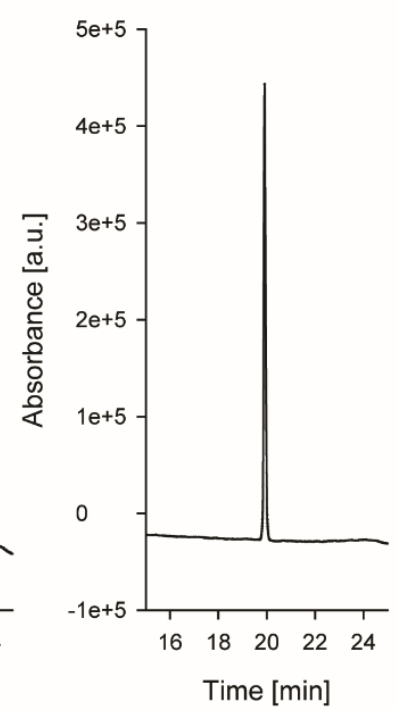

Figure A20: Verification of purity by HPLC analysis of Chromis-PGA-(PEG10)1-Cy5. a) Fluorescence detection $\left.\left(\lambda_{\mathrm{ex}}=488 \mathrm{~nm}, \lambda_{\mathrm{em}}=525 \mathrm{~nm}\right) \mathrm{b}\right)$ fluorescence detection $\left(\lambda_{\mathrm{ex}}=620 \mathrm{~nm}\right.$, $\lambda_{\mathrm{em}}=665 \mathrm{~nm}$ ) c) absorbance at $500 \mathrm{~nm} \mathrm{~d}$ ) absorbance at $650 \mathrm{~nm}$. 


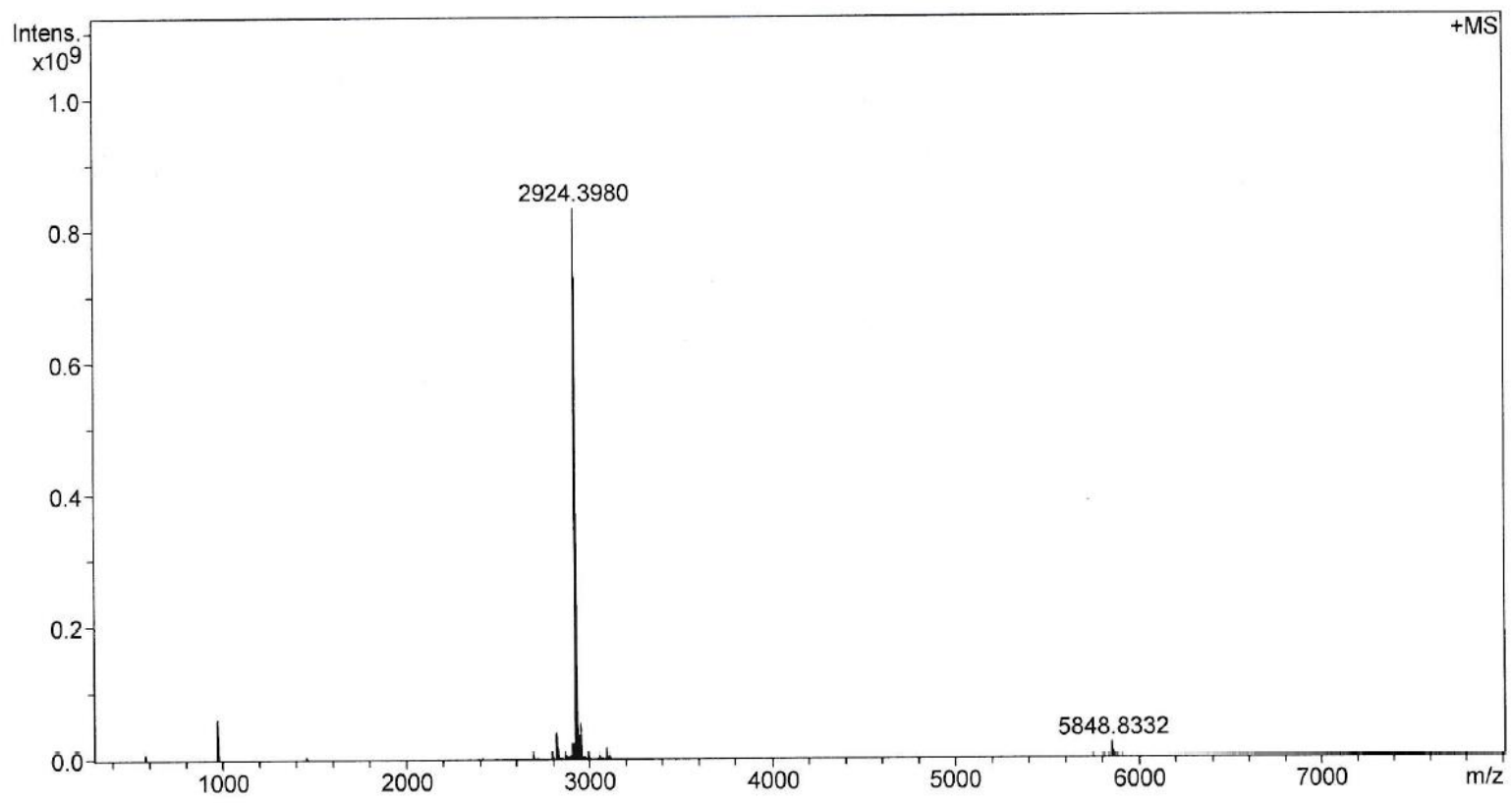

Figure A21: HRMS-MALDI of Chromis-PGA-(PEG10)1-Cy5.
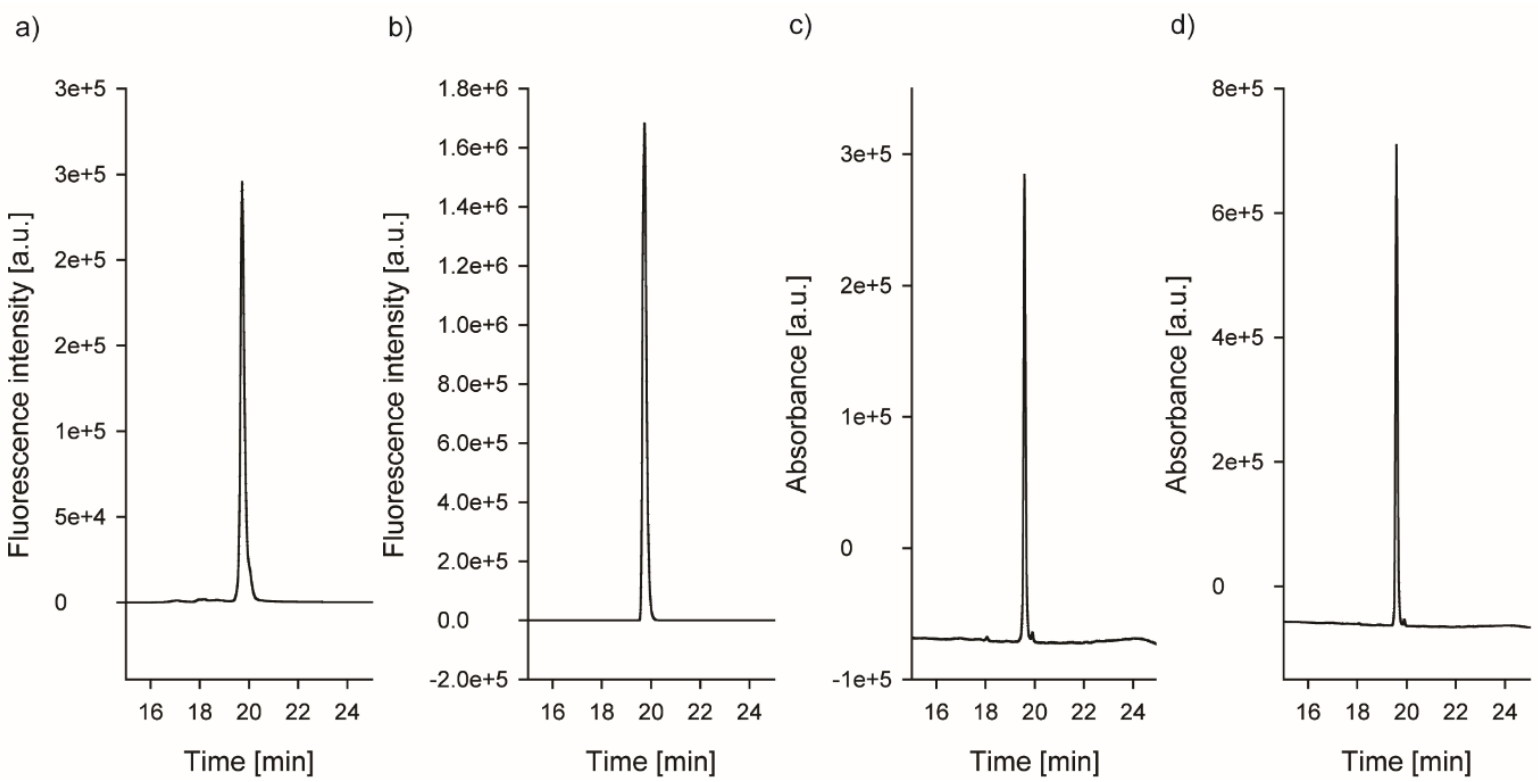

Figure A22: Verification of purity by HPLC analysis of Chromis-PGA-(PEG10)2-Cy5. a) Fluorescence detection $\left.\left(\lambda_{\mathrm{ex}}=488 \mathrm{~nm}, \lambda_{\mathrm{em}}=525 \mathrm{~nm}\right) \mathrm{b}\right)$ fluorescence detection $\left(\lambda_{\mathrm{ex}}=620 \mathrm{~nm}\right.$, $\lambda_{\mathrm{em}}=665 \mathrm{~nm}$ ) c) absorbance at $500 \mathrm{~nm} \mathrm{~d}$ ) absorbance at $650 \mathrm{~nm}$. 


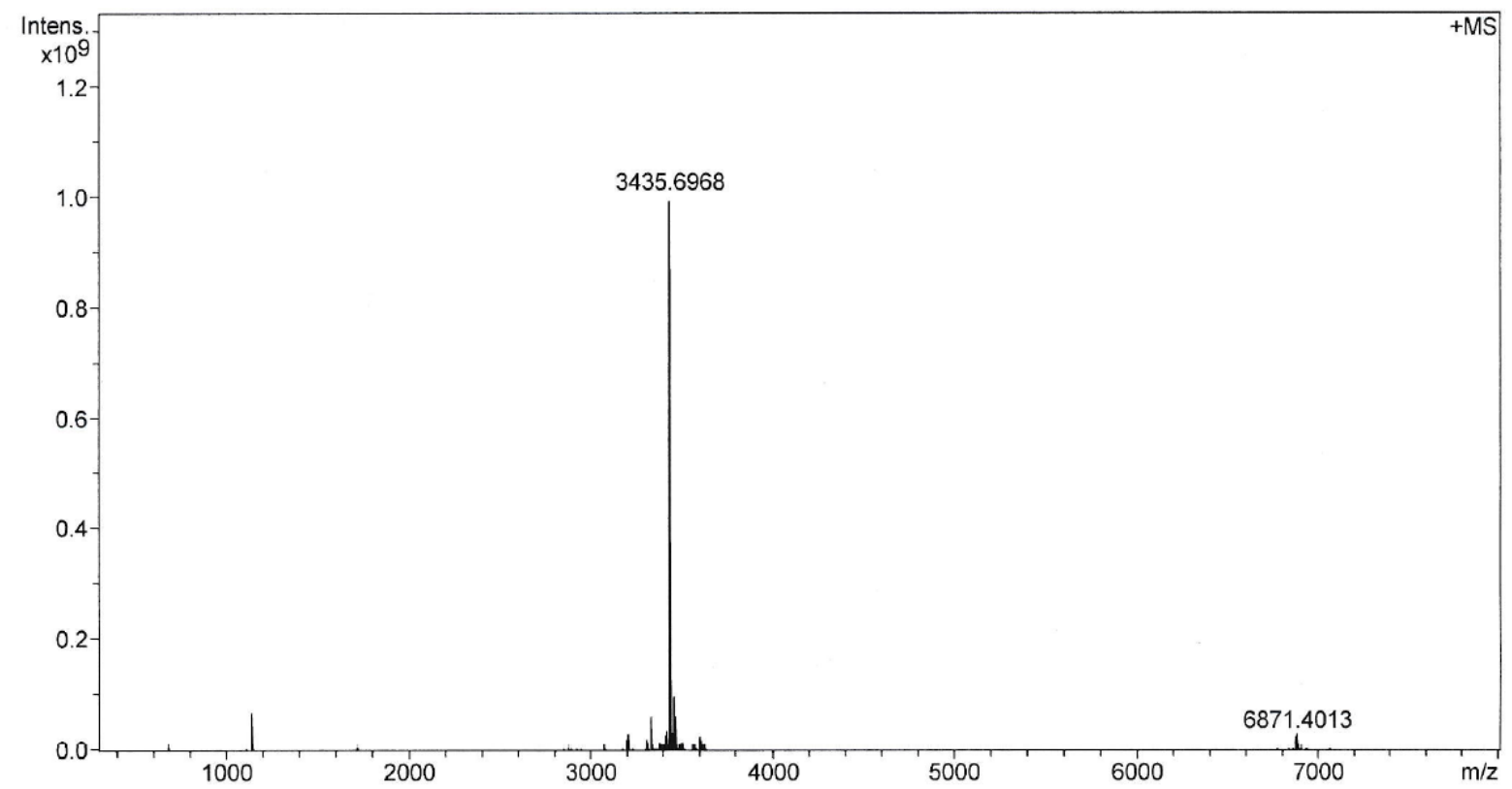

Figure A23: HRMS-MALDI of Chromis-PGA-(PEG10)2-Cy5.
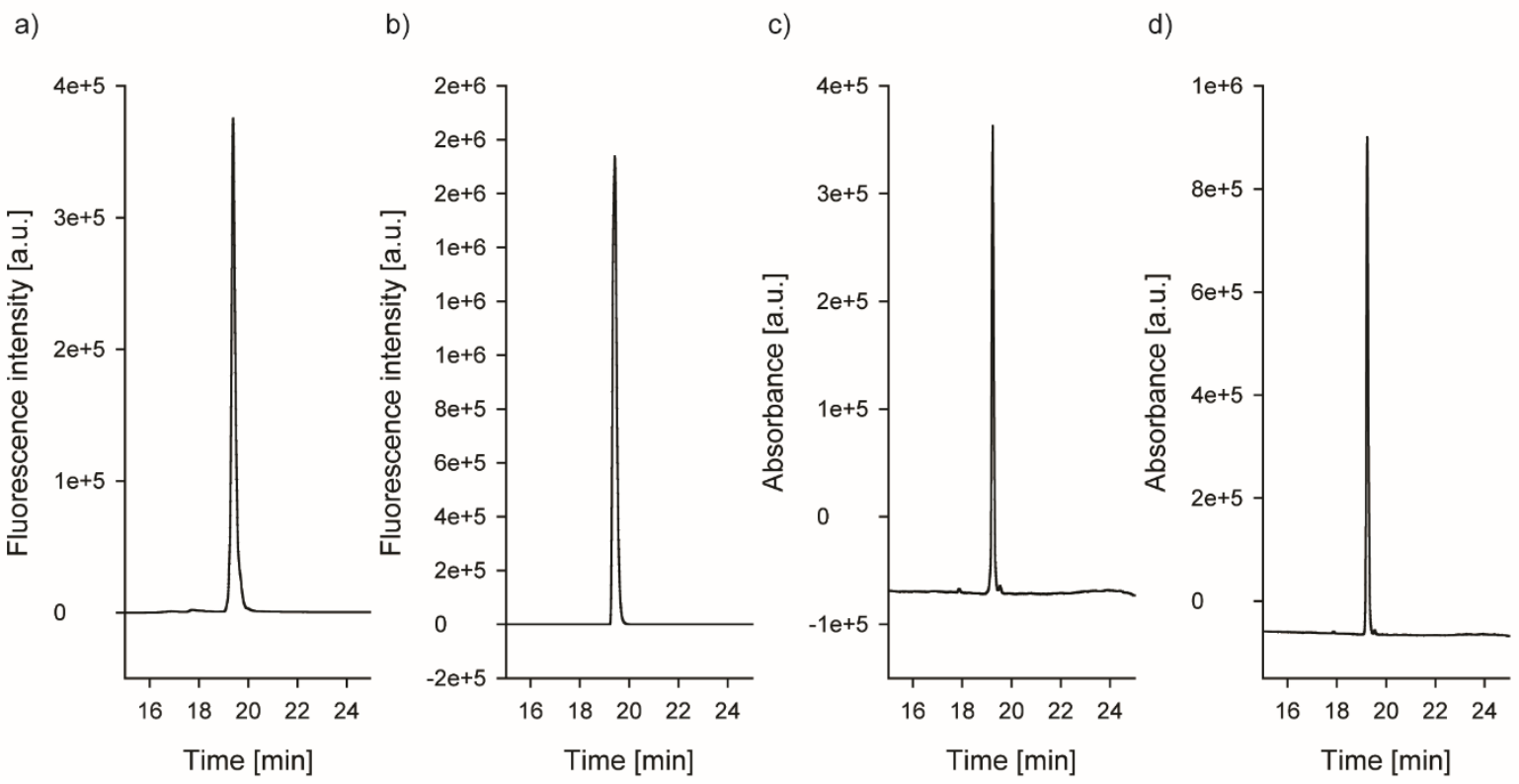

Figure A24: Verification of purity by HPLC analysis of Chromis-PGA-(PEG 10 $_{3}-\mathrm{Cy}_{2}$. a) Fluorescence detection $\left.\left(\lambda_{\mathrm{ex}}=488 \mathrm{~nm}, \lambda_{\mathrm{em}}=525 \mathrm{~nm}\right) \mathrm{b}\right)$ fluorescence detection $\left(\lambda_{\mathrm{ex}}=620 \mathrm{~nm}\right.$, $\left.\lambda_{\mathrm{em}}=665 \mathrm{~nm}\right) \mathrm{c}$ ) absorbance at $500 \mathrm{~nm} \mathrm{~d}$ ) absorbance at $650 \mathrm{~nm}$. 


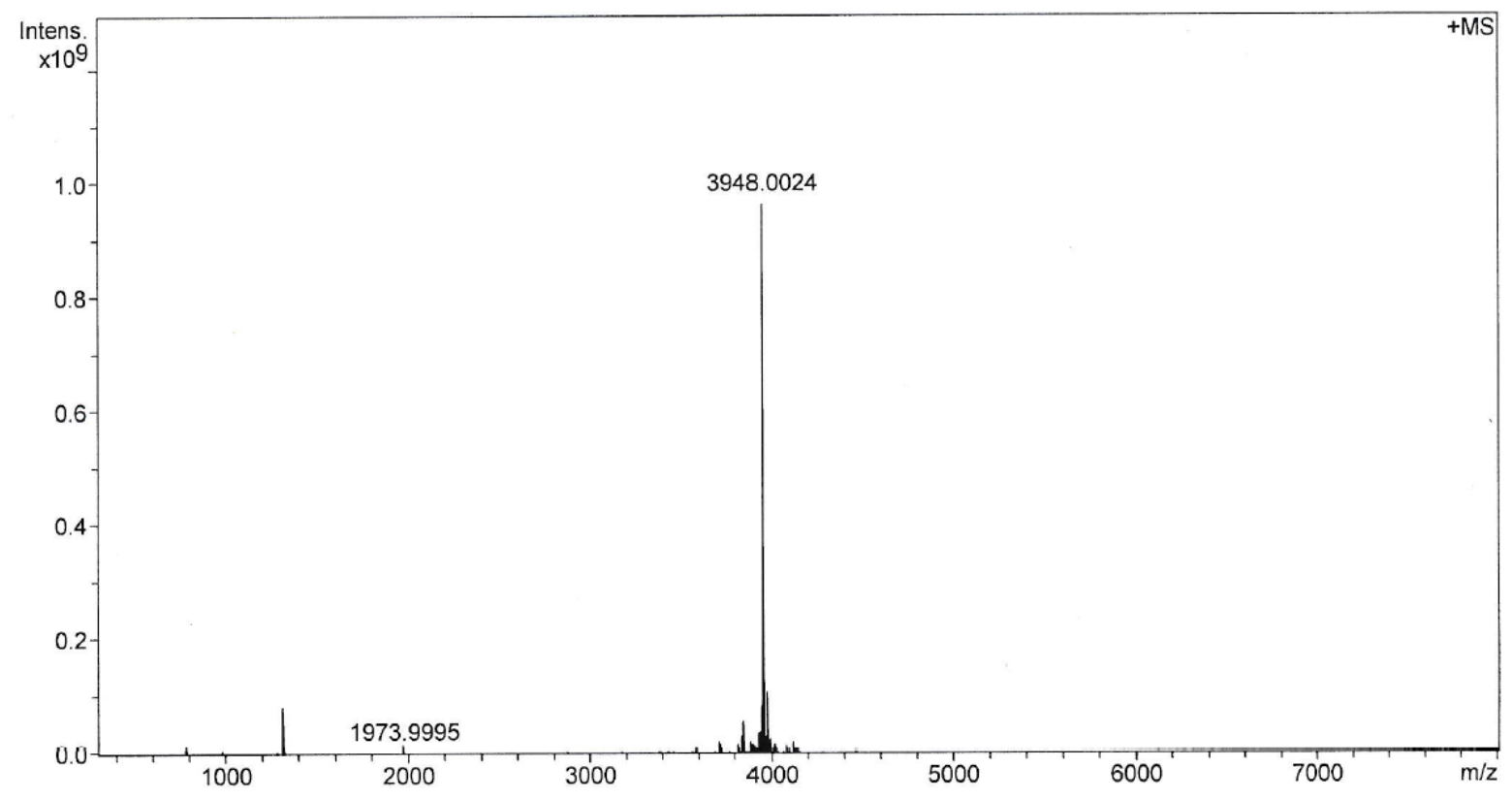

Figure A25: HRMS-MALDI of Chromis-PGA-(PEG10)3-Cy5.

a)

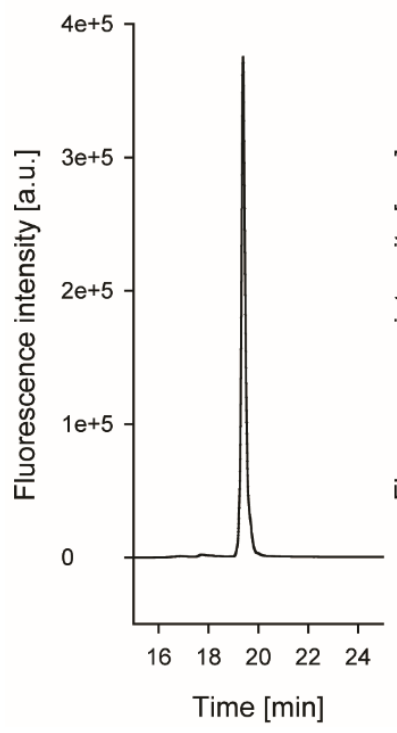

b)

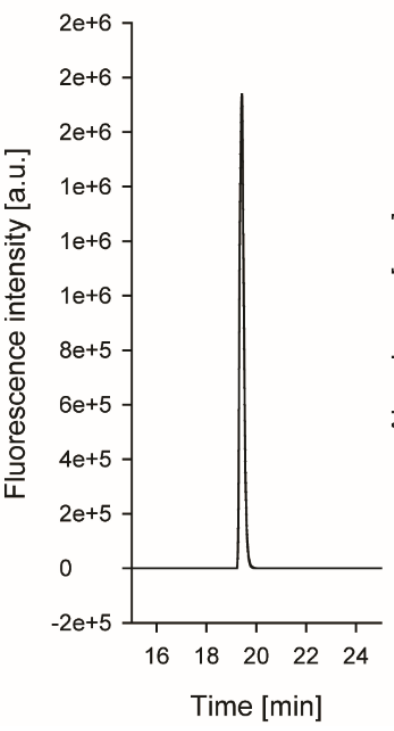

c)

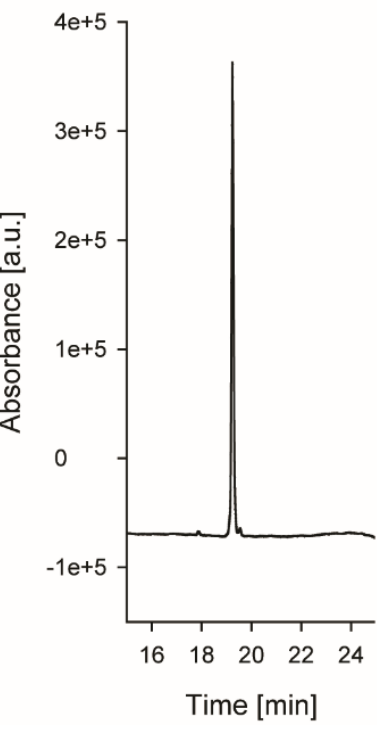

d)

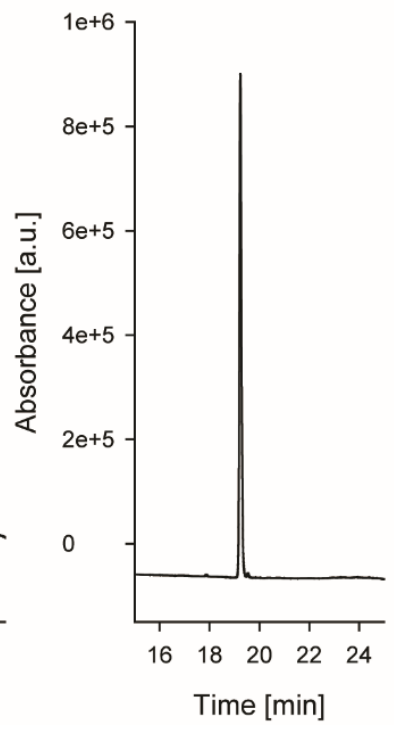

Figure A26: Verification of purity by HPLC analysis of Chromis-PGA-(PEG 10$)_{4}-\mathrm{Cy} 5$. a) Fluorescence detection $\left.\left(\lambda_{\mathrm{ex}}=488 \mathrm{~nm}, \lambda_{\mathrm{em}}=525 \mathrm{~nm}\right) \mathrm{b}\right)$ fluorescence detection $\left(\lambda_{\mathrm{ex}}=620 \mathrm{~nm}\right.$, $\left.\lambda_{\mathrm{em}}=665 \mathrm{~nm}\right) \mathrm{c}$ ) absorbance at $500 \mathrm{~nm} \mathrm{~d}$ ) absorbance at $650 \mathrm{~nm}$. 


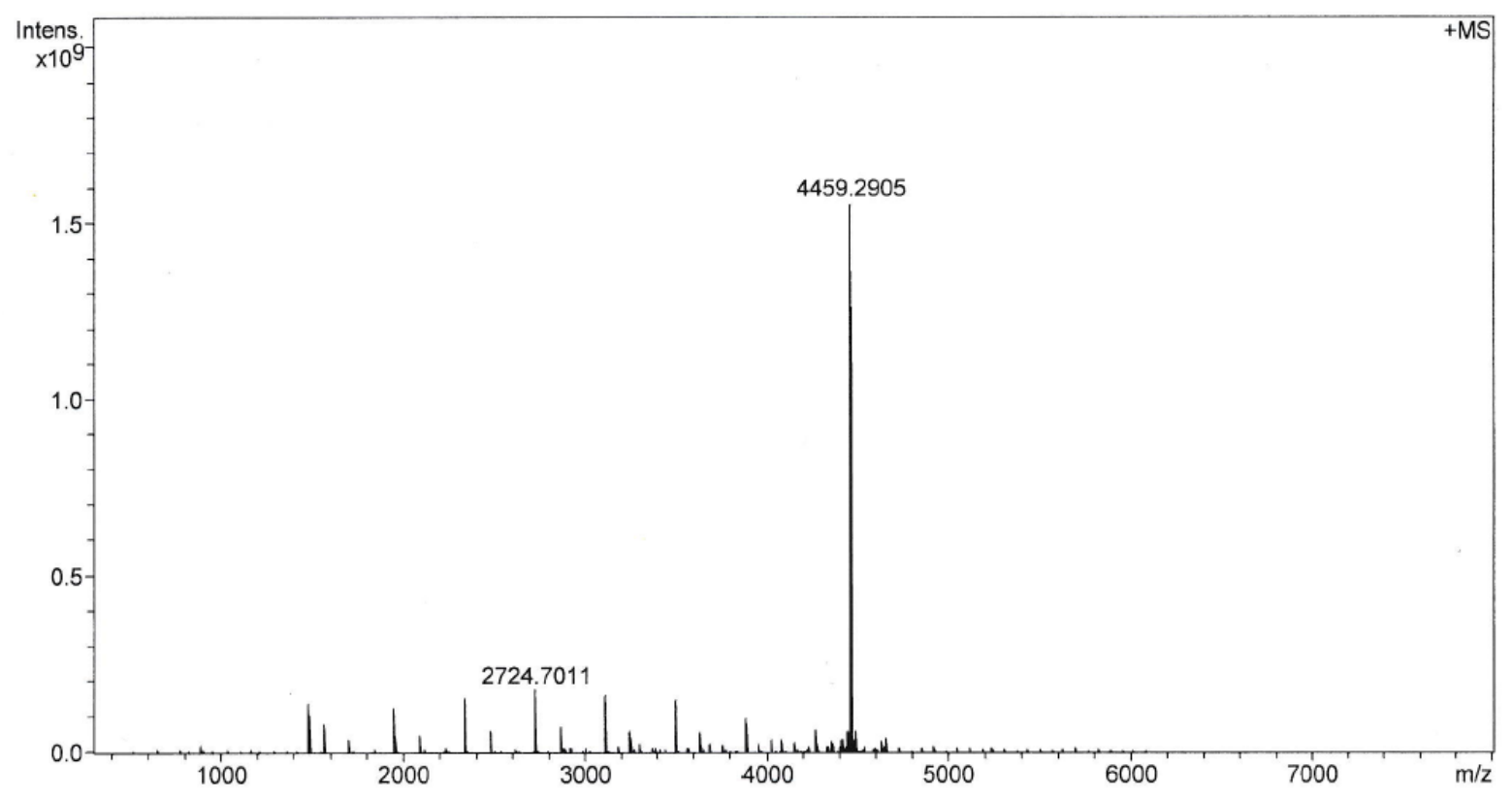

Figure A27: HRMS-MALDI of Chromis-PGA-(PEG10)4-Cy5.
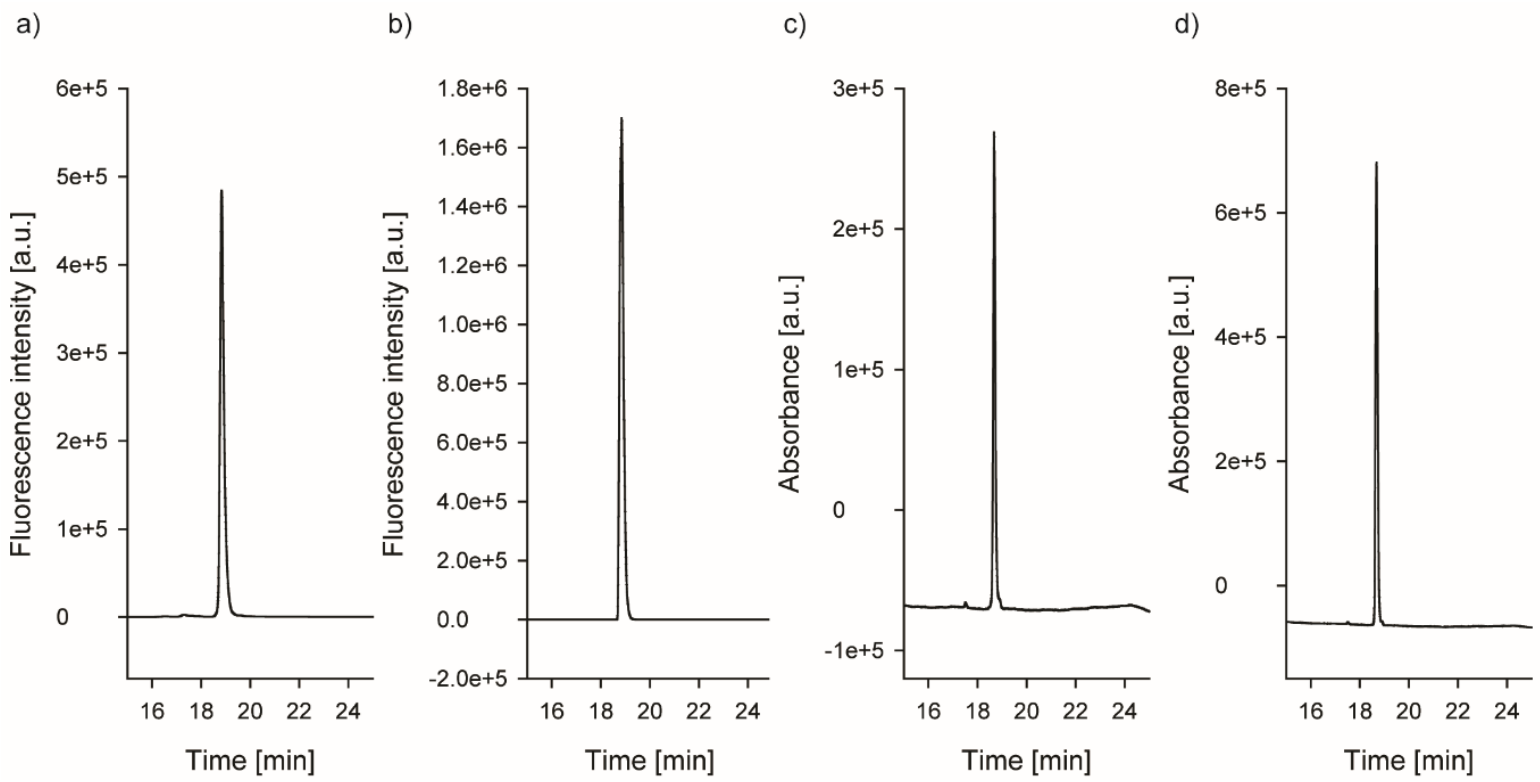

Figure A28: Verification of purity by HPLC analysis of Chromis-PGA-(PEG 10$)_{5}-\mathrm{Cy} 5$. a) Fluorescence detection $\left.\left(\lambda_{\mathrm{ex}}=488 \mathrm{~nm}, \lambda_{\mathrm{em}}=525 \mathrm{~nm}\right) \mathrm{b}\right)$ fluorescence detection $\left(\lambda_{\mathrm{ex}}=620 \mathrm{~nm}\right.$, $\lambda_{\mathrm{em}}=665 \mathrm{~nm}$ ) c) absorbance at $500 \mathrm{~nm} \mathrm{~d}$ ) absorbance at $650 \mathrm{~nm}$. 


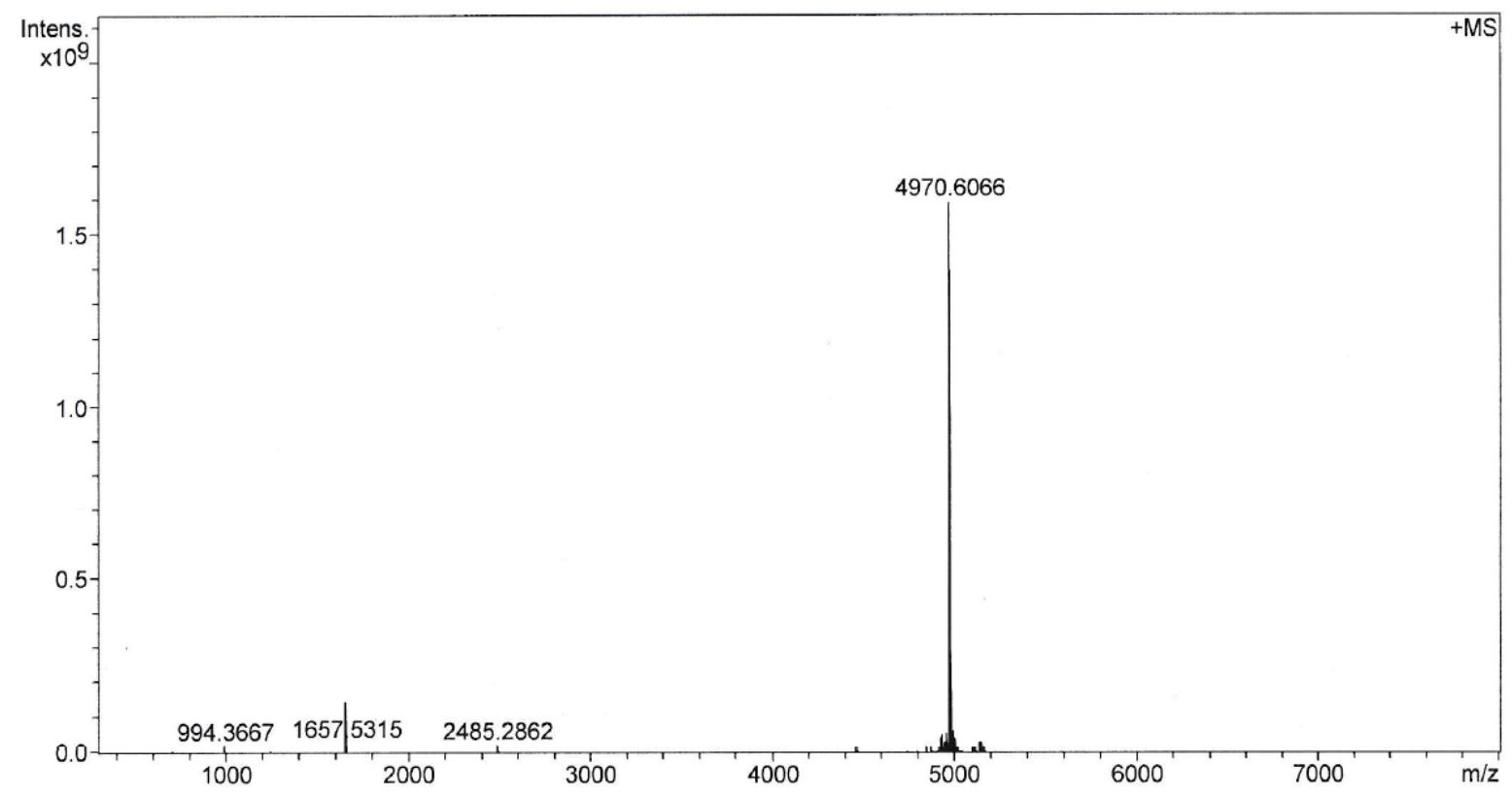

Figure A29: HRMS-MALDI of Chromis-PGA-(PEG10)5-Cy5.

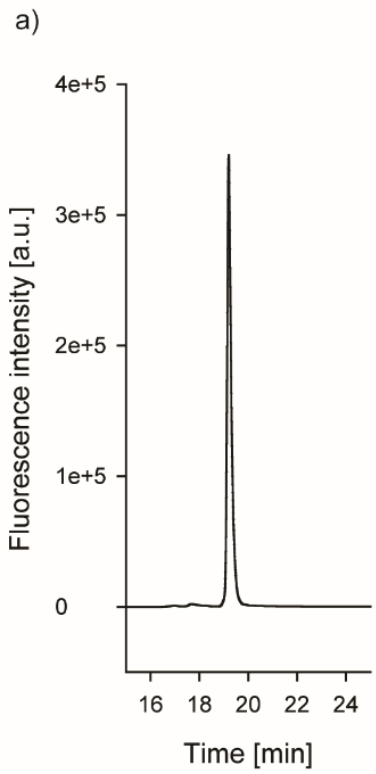

b)

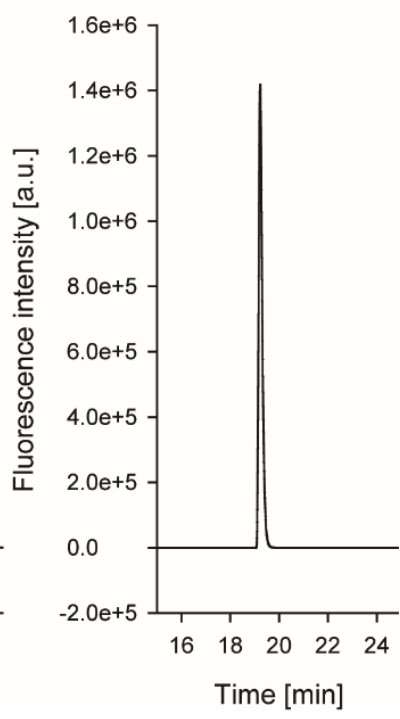

c)

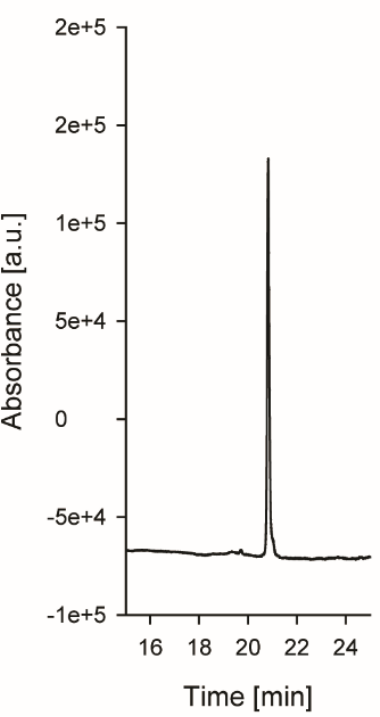

d)

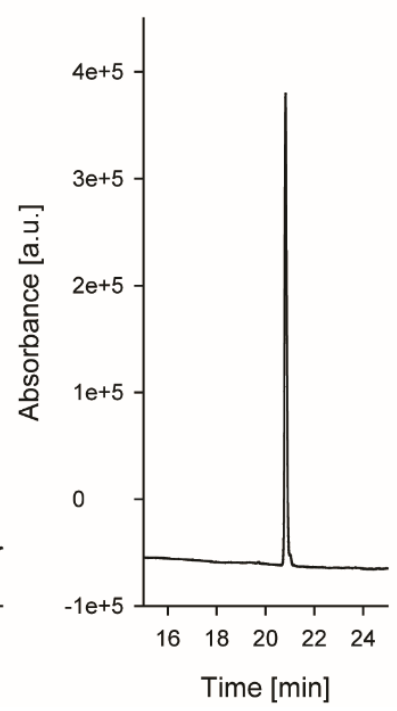

Figure A30: Verification of purity by HPLC analysis of Chromis-PGA-(PEG10)6-Cy5. a) Fluorescence detection $\left.\left(\lambda_{\mathrm{ex}}=488 \mathrm{~nm}, \lambda_{\mathrm{em}}=525 \mathrm{~nm}\right) \mathrm{b}\right)$ fluorescence detection $\left(\lambda_{\mathrm{ex}}=620 \mathrm{~nm}\right.$, $\lambda_{\mathrm{em}}=665 \mathrm{~nm}$ ) c) absorbance at $500 \mathrm{~nm} \mathrm{~d}$ ) absorbance at $650 \mathrm{~nm}$. 


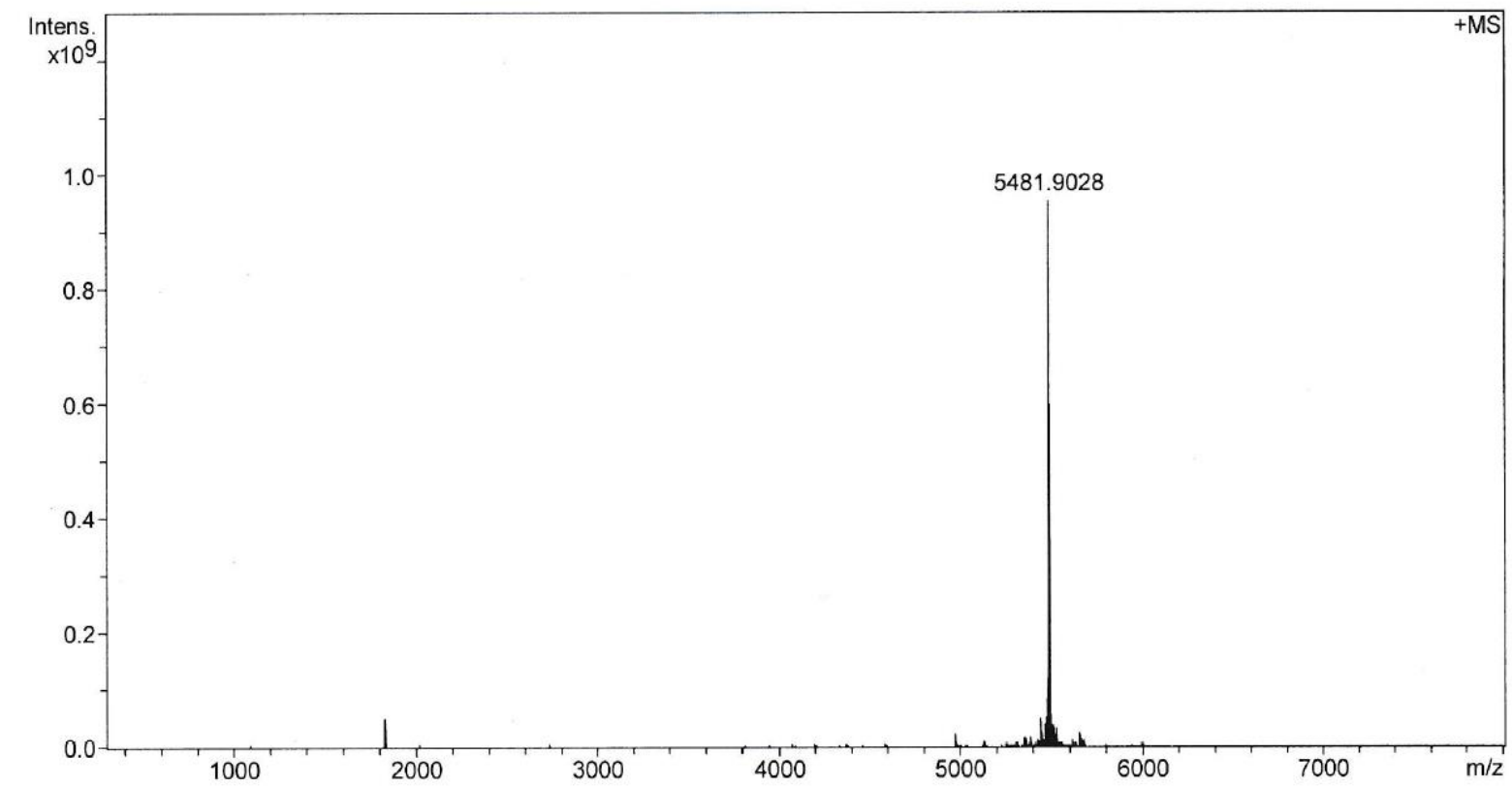

Figure A31: HRMS-MALDI of Chromis-PGA-( $\left.\mathrm{PEG}_{10}\right)_{6}-\mathrm{Cy} 5$. 


\section{Characterization of molecules in Chapter 5}

\section{Lys-PNA}
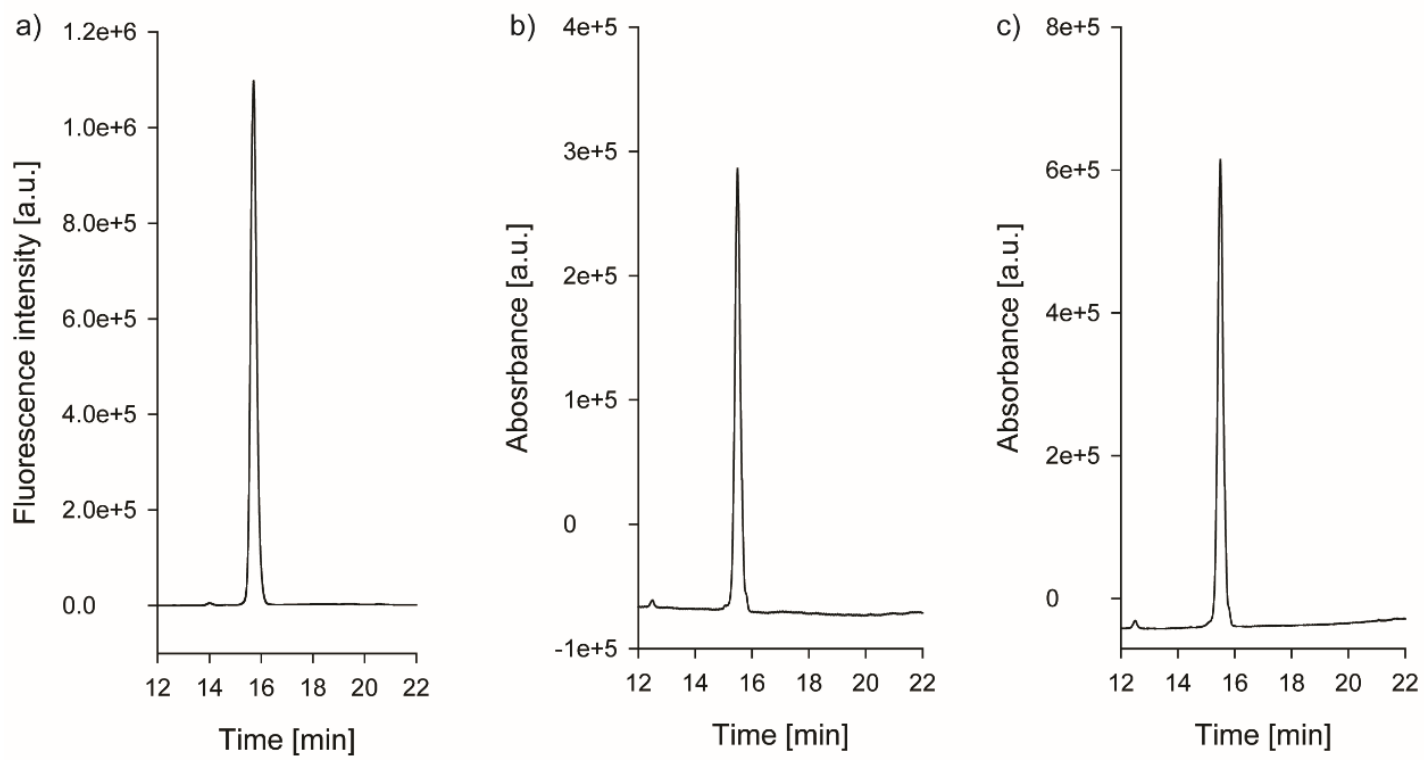

Figure A32: Verification of purity by HPLC analysis of PNA-Chromis500. a) Fluorescence detection $\left(\lambda_{\mathrm{ex}}=488 \mathrm{~nm}, \lambda_{\mathrm{em}}=525 \mathrm{~nm}\right) \mathrm{b}$ ) absorbance at $500 \mathrm{~nm} \mathrm{c}$ ) absorbance at $260 \mathrm{~nm}$.

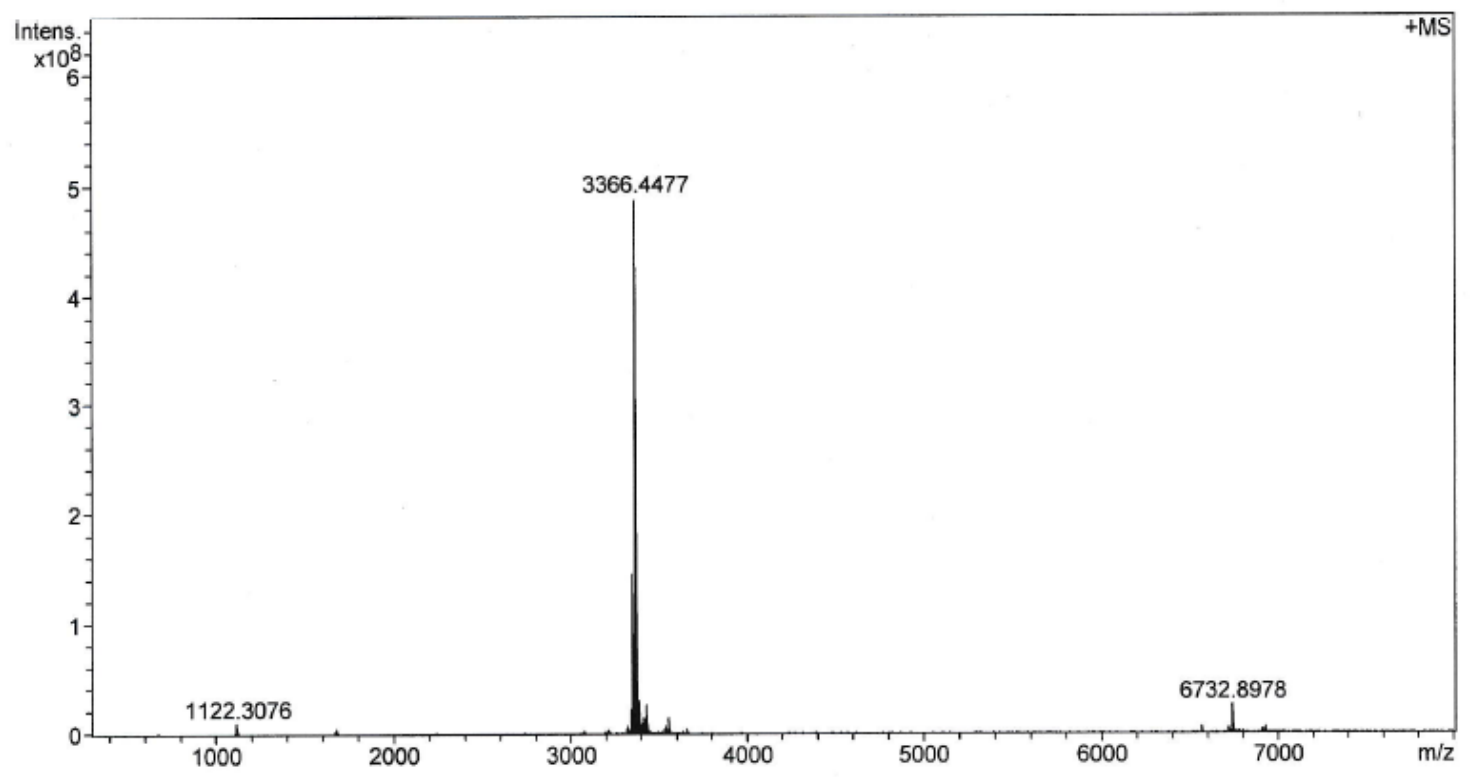

Figure A33: HRMS MALDI of PNA-Chromis500. 


\section{Protected peptide-cyanine conjugate}

Table A3: Expected m/z of Ala-Glu( $\left.{ }^{\mathrm{t}} \mathrm{Bu}\right)_{10}-\left(\mathrm{PEG}_{10}\right)_{0-6}-\mathrm{Cy} 5$.

\begin{tabular}{|c|c|}
\hline$\left(\mathrm{PEG}_{10}\right)_{\mathrm{x}}-\mathrm{Cy} 5$ & $\mathrm{MW}[\mathrm{g} / \mathrm{mol}]$ \\
\hline$\left(\mathrm{PEG}_{10}\right)_{0}-\mathrm{Cy} 5$ & 2406.98 \\
\hline$\left(\mathrm{PEG}_{10}\right)_{1}-\mathrm{Cy} 5$ & 2918.59 \\
\hline$\left(\mathrm{PEG}_{10}\right)_{2}-\mathrm{Cy} 5$ & 3430.20 \\
\hline$\left(\mathrm{PEG}_{10}\right)_{3}-\mathrm{Cy} 5$ & 3941.81 \\
\hline$\left(\mathrm{PEG}_{10}\right)_{4}-\mathrm{Cy} 5$ & 4453.42 \\
& \\
\hline
\end{tabular}

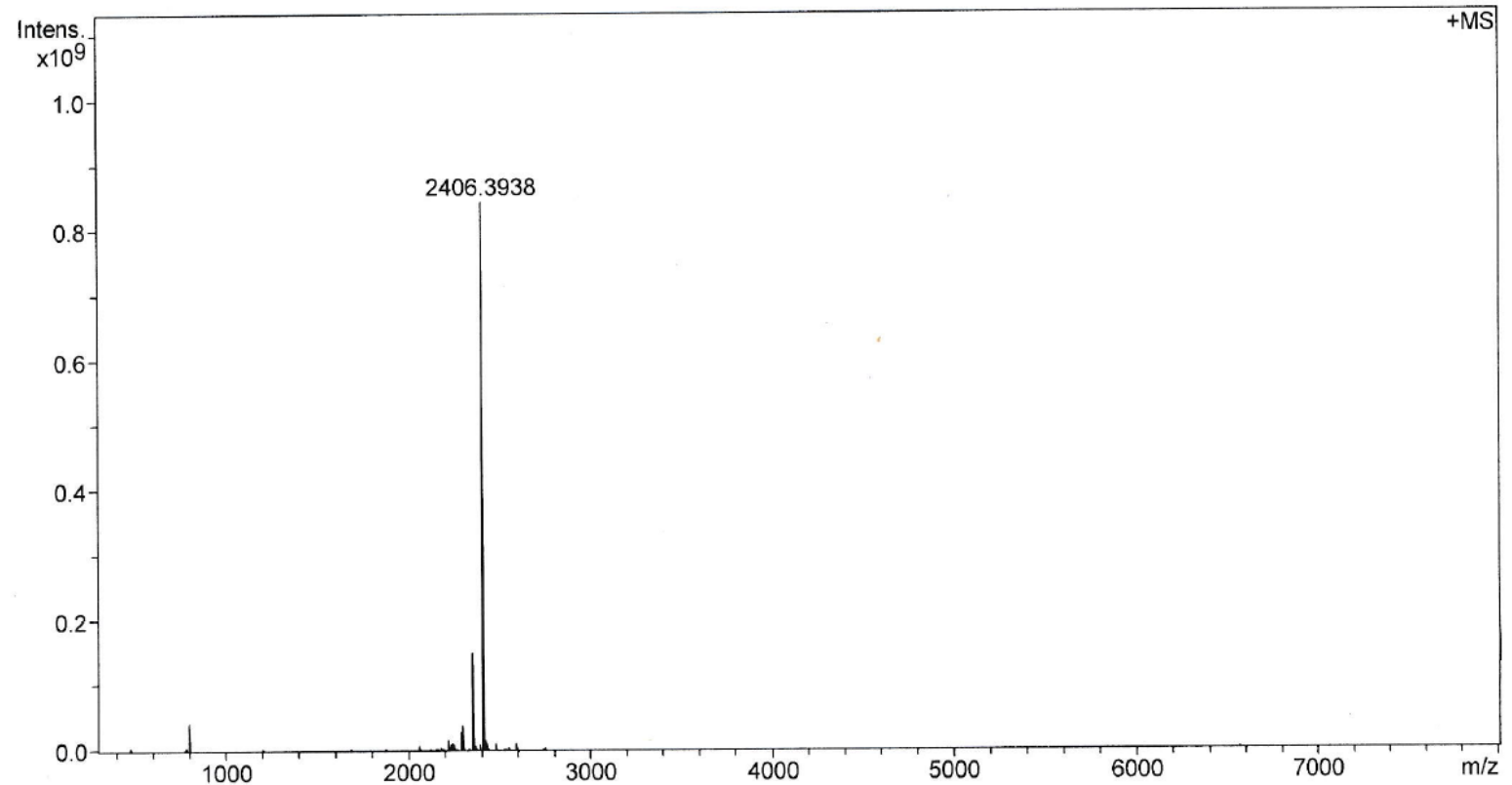

Figure A34: HRMS-MALDI of HO-Ala-Glu $10\left({ }^{\mathrm{t}} \mathrm{Bu}\right)-\left(\mathrm{PEG}_{10}\right)_{0}-\mathrm{Cy} 5$. 


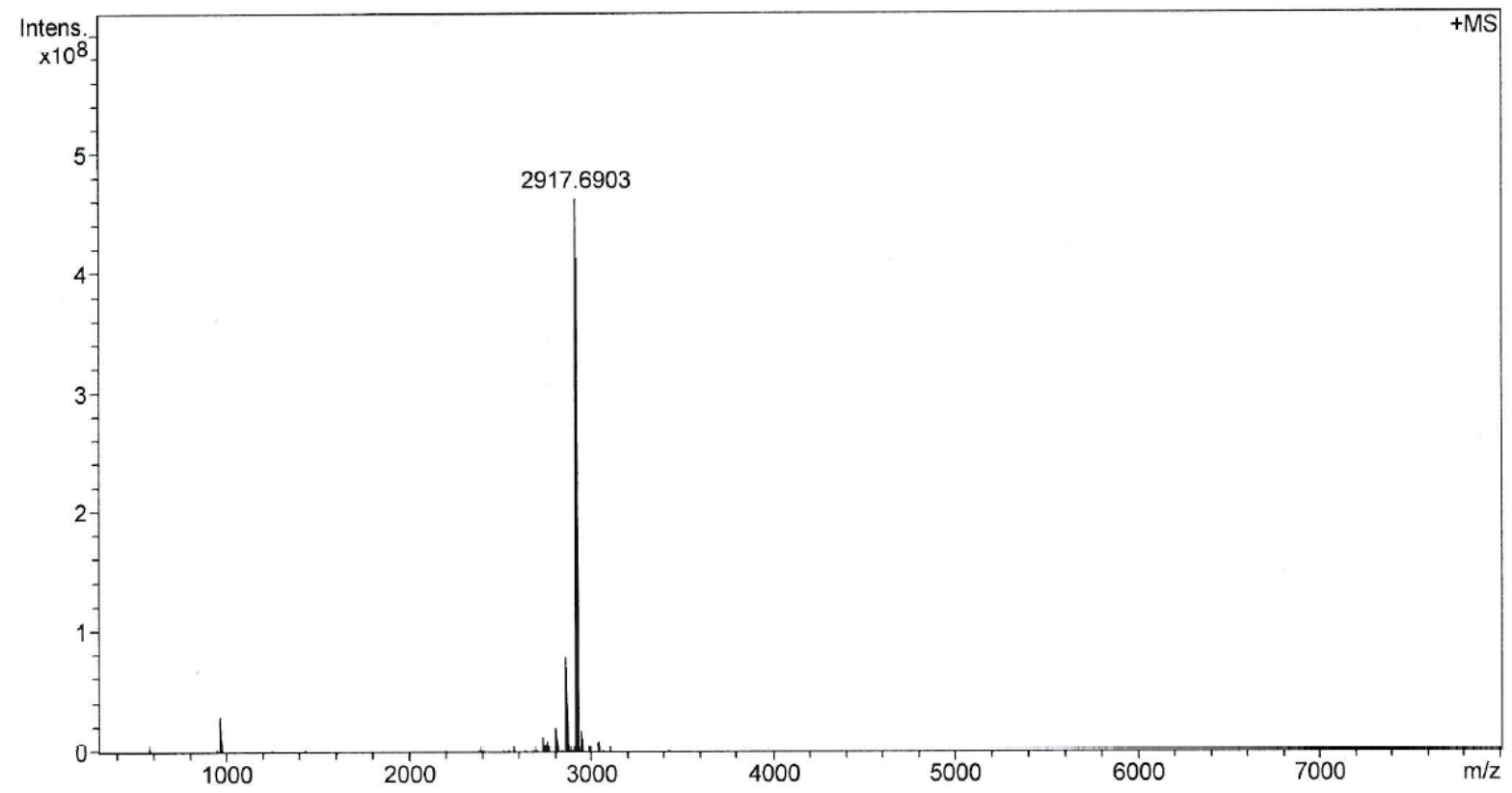

Figure A35: HRMS-MALDI of HO-Ala-Glu( $\left.{ }^{\mathrm{t} B u}\right)_{10}-\left(\mathrm{PEG}_{10}\right)_{1}-\mathrm{Cy} 5$.

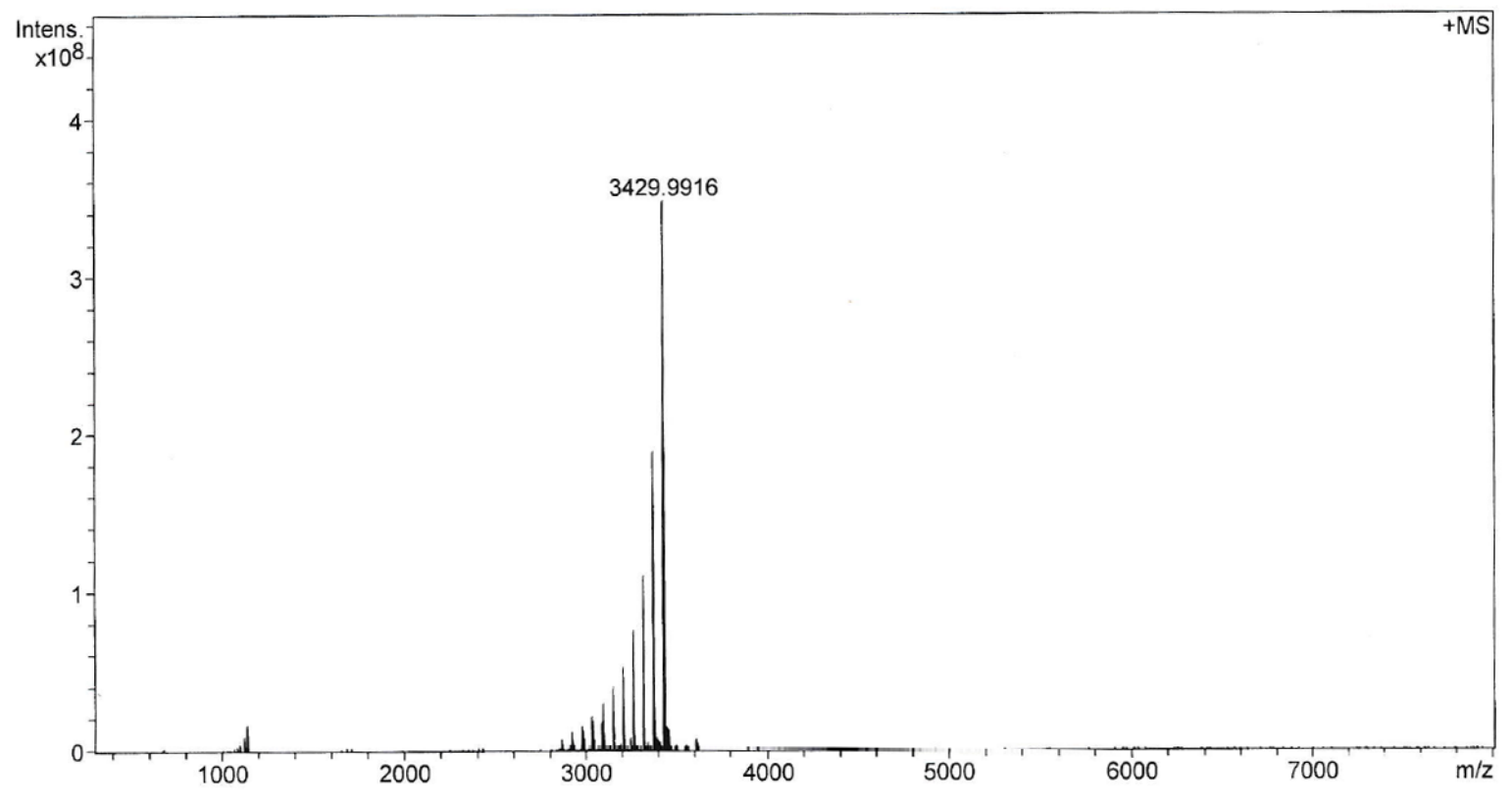

Figure A36: HRMS-MALDI of HO-Ala-Glu( $\left.{ }^{\mathrm{t}} \mathrm{Bu}\right)_{10-}\left(\mathrm{PEG}_{10}\right)_{2}-\mathrm{Cy} 5$. 


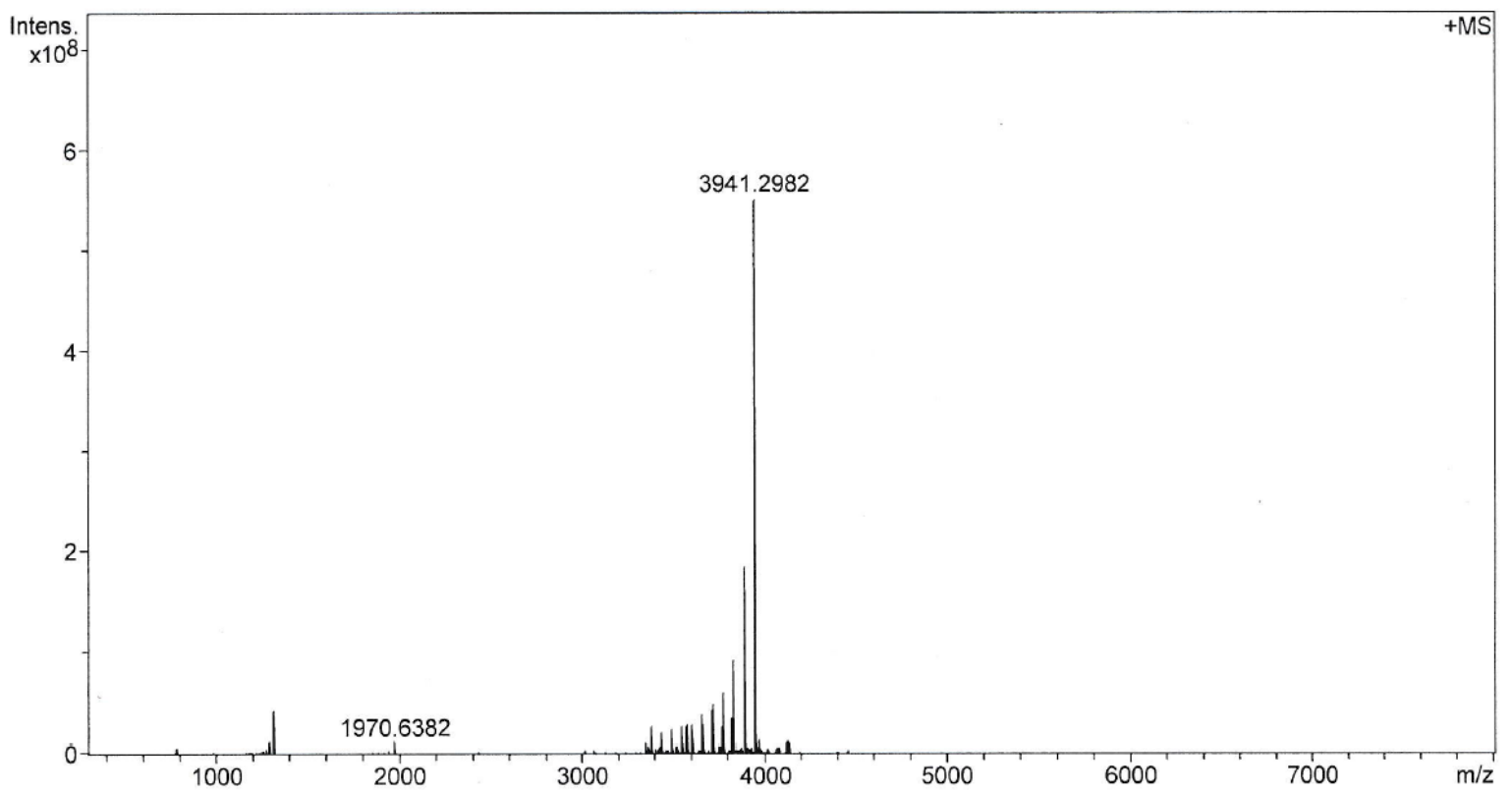

Figure A37: HRMS-MALDI of HO-Ala-Glu( $\left.{ }^{\mathrm{t}} \mathrm{Bu}\right){ }_{10}-\left(\mathrm{PEG}_{10}\right)_{3}-\mathrm{Cy} 5$.

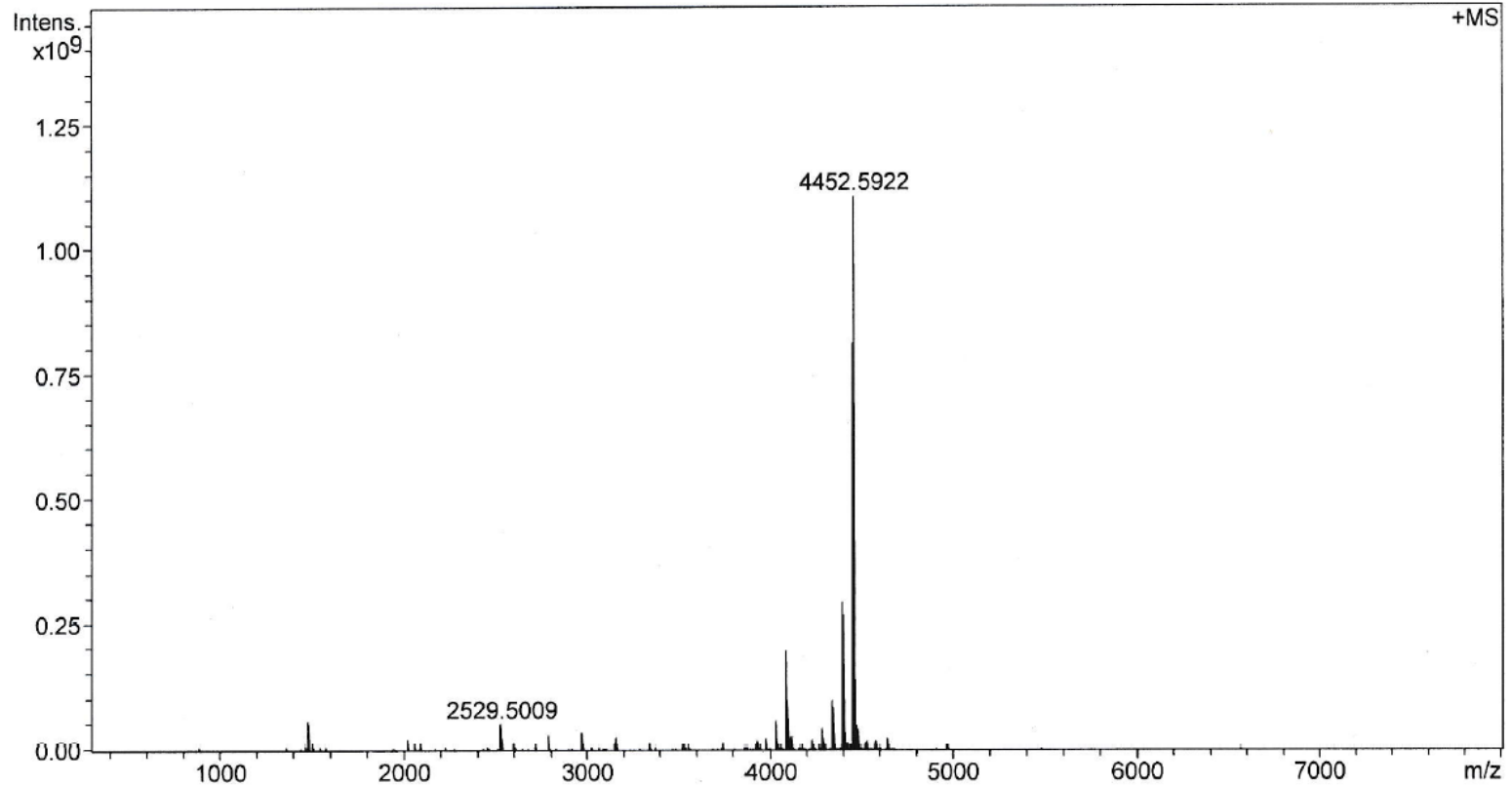

Figure A38: HRMS-MALDI of HO-Ala-Glu( $\left.{ }^{\mathrm{t} B u}\right)_{10}-\left(\mathrm{PEG}_{10}\right)_{4}-\mathrm{Cy} 5$. 
PNA-Ala-Glu $10-\left(\mathrm{PEG}_{10}\right)_{\mathrm{x}}-\mathrm{Cy} 5$ conjugates

Table A4:Expected m/z of PNA-PEG $0-4-\mathrm{Cy} 5$

\begin{tabular}{|c|c|}
\hline PNA-(PEG 10$)_{x}-$ Cy5 & $\mathrm{MW}[\mathrm{g} / \mathrm{mol}]$ \\
\hline PNA-(PEG 10$)_{0}-\mathrm{Cy} 5$ & 4684.69 \\
\hline PNA-(PEG 10$)_{1}-\mathrm{Cy} 5$ & 5196.30 \\
\hline PNA-(PEG 10$)_{2}-\mathrm{Cy} 5$ & 5707.91 \\
\hline PNA-( PEG $\left._{10}\right)_{3}-\mathrm{Cy} 5$ & 6219.52 \\
\hline PNA-(PEG 10$)_{4}-\mathrm{Cy} 5$ & 6731.12 \\
\hline
\end{tabular}
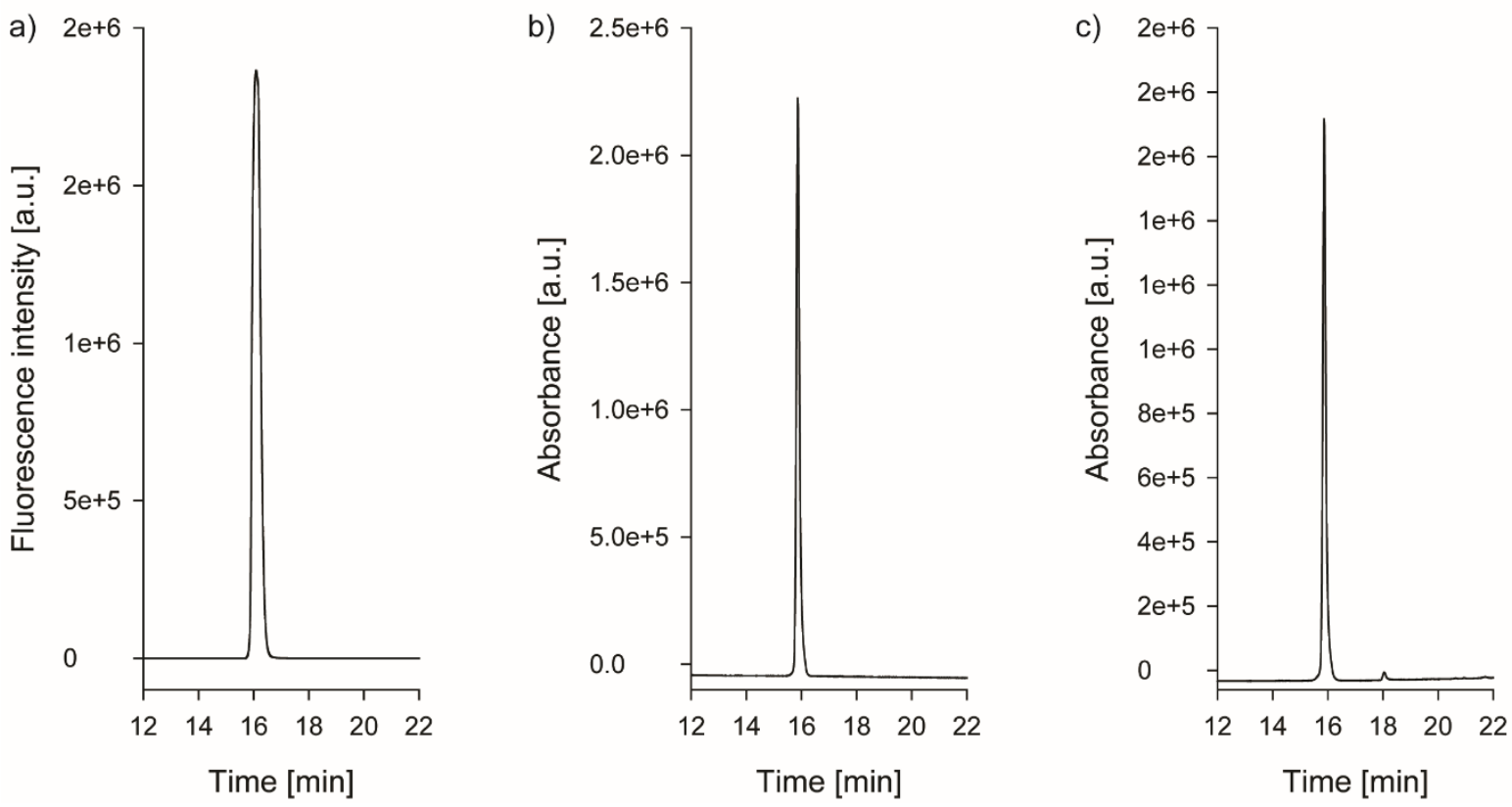

Figure A39: Verification of purity by HPLC analysis of PNA-(PEG10)0-Cy5. a) Fluorescence detection $\left(\lambda_{\mathrm{ex}}=620 \mathrm{~nm}, \lambda_{\mathrm{em}}=665 \mathrm{~nm}\right)$ b) absorbance at $\left.640 \mathrm{~nm} \mathrm{c}\right)$ absorbance at $260 \mathrm{~nm}$. 


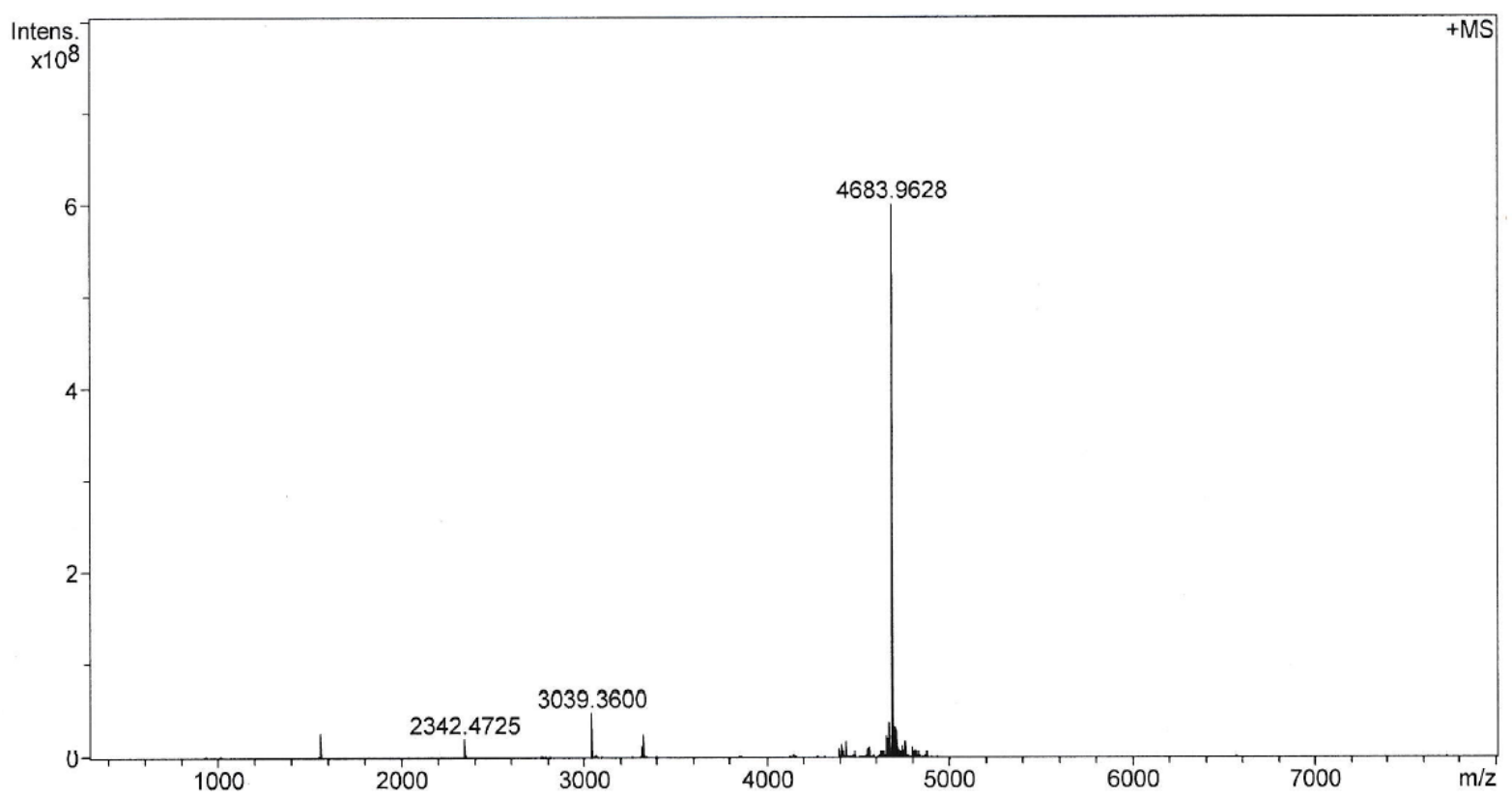

Figure A40: HRMS MALDI of PNA-(PEG 10$)_{0}-\mathrm{Cy} 5$.
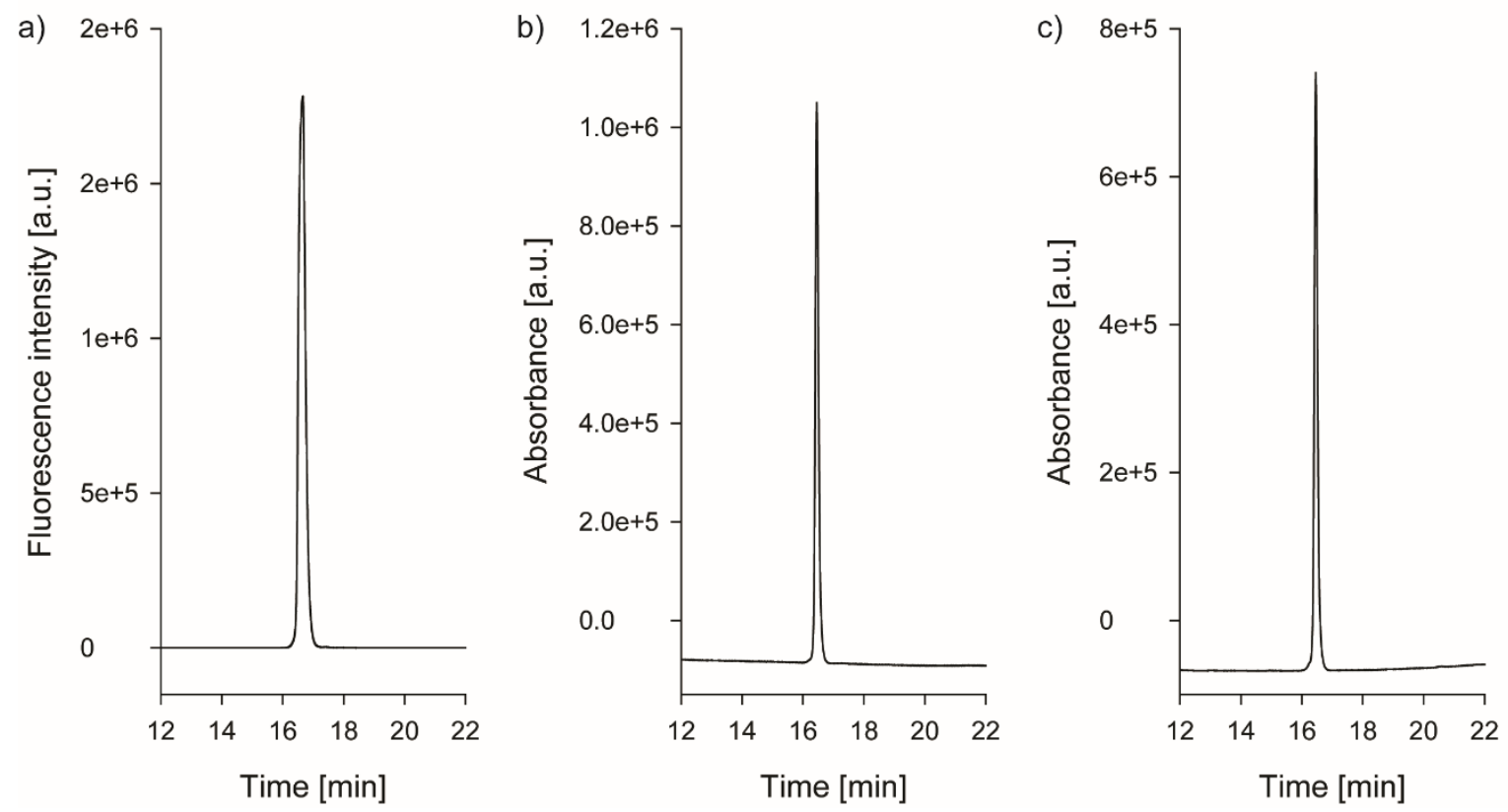

Figure A41: Verification of purity by HPLC analysis of PNA-(PEG 10$)_{1}-\mathrm{Cy} 5$. a) Fluorescence detection $\left(\lambda_{\mathrm{ex}}=620 \mathrm{~nm}, \lambda_{\mathrm{em}}=665 \mathrm{~nm}\right)$ b) absorbance at $\left.640 \mathrm{~nm} \mathrm{c}\right)$ absorbance at $260 \mathrm{~nm}$. 


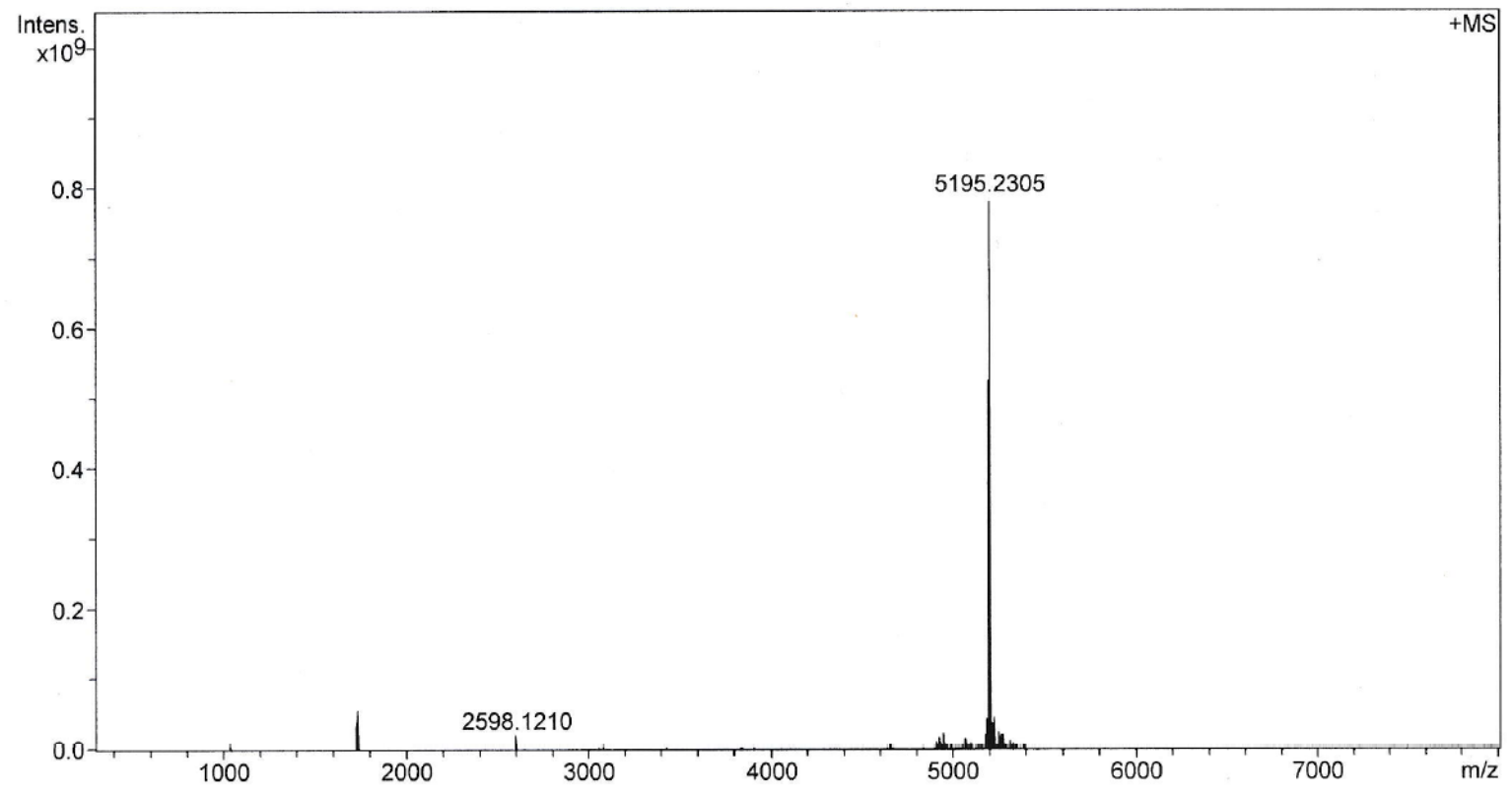

Figure A42: HRMS-MALDI of PNA-(PEG10) ${ }_{1}-\mathrm{Cy} 5$.
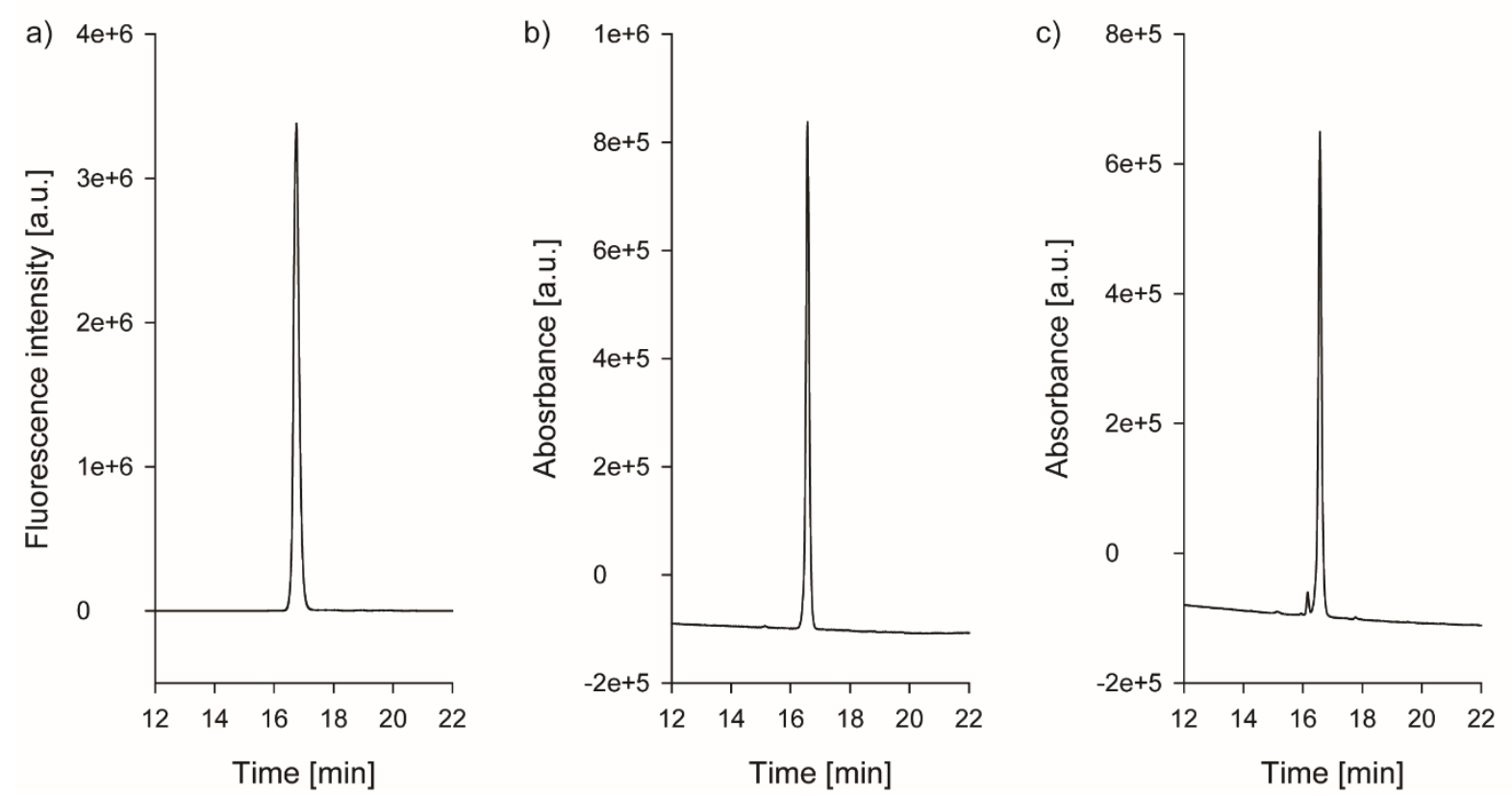

Figure A43: Verification of purity by HPLC analysis of PNA-( $\left.\mathrm{PEG}_{10}\right)_{2}-\mathrm{Cy} 5$. a) Fluorescence detection $\left(\lambda_{\mathrm{ex}}=620 \mathrm{~nm}, \lambda_{\mathrm{em}}=665 \mathrm{~nm}\right) \mathrm{b}$ ) absorbance at $\left.640 \mathrm{~nm} \mathrm{c}\right)$ absorbance at $260 \mathrm{~nm}$. 


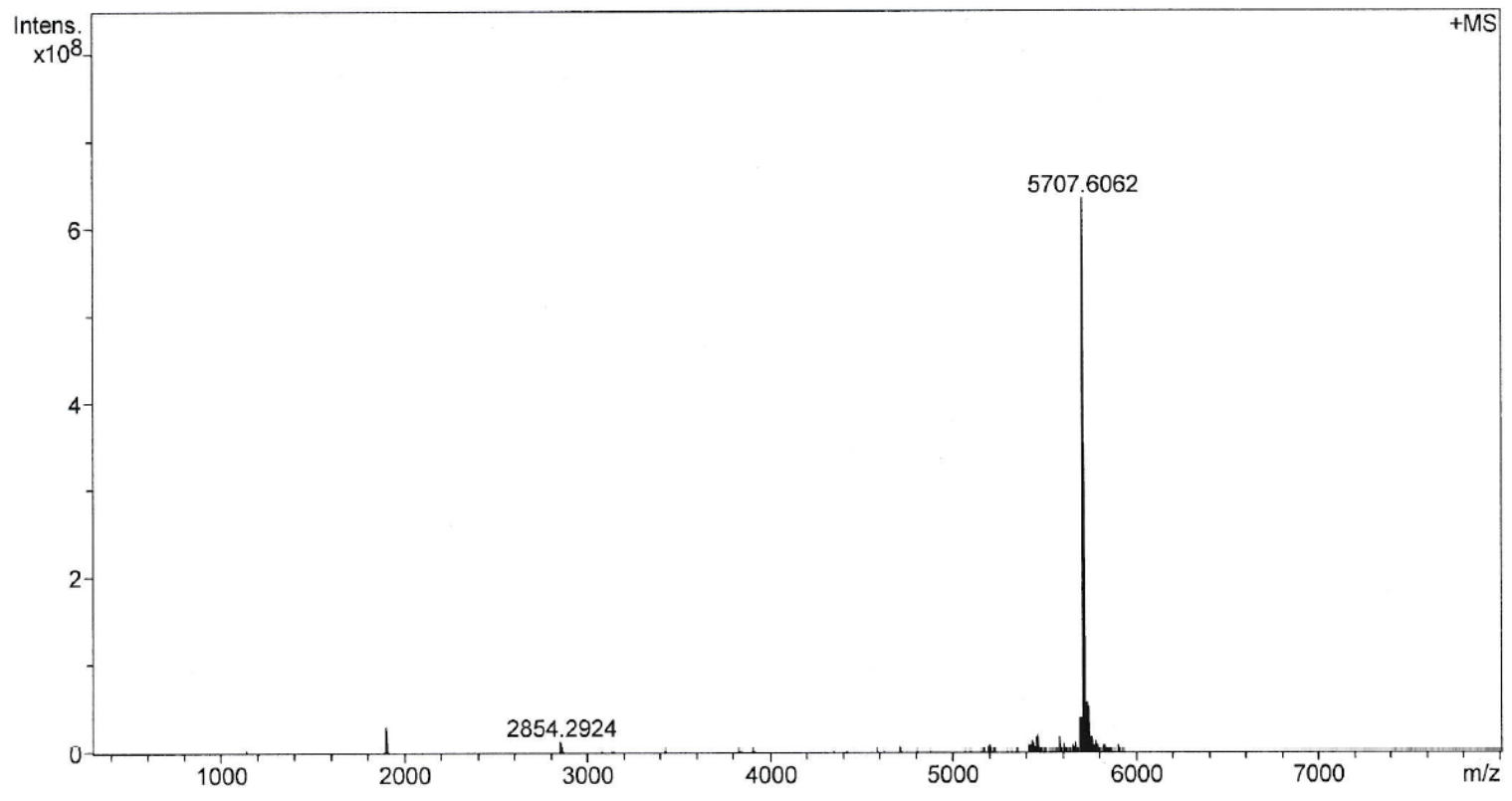

Figure A44: HRMS-MALDI of PNA-(PEG10)2-Cy5.
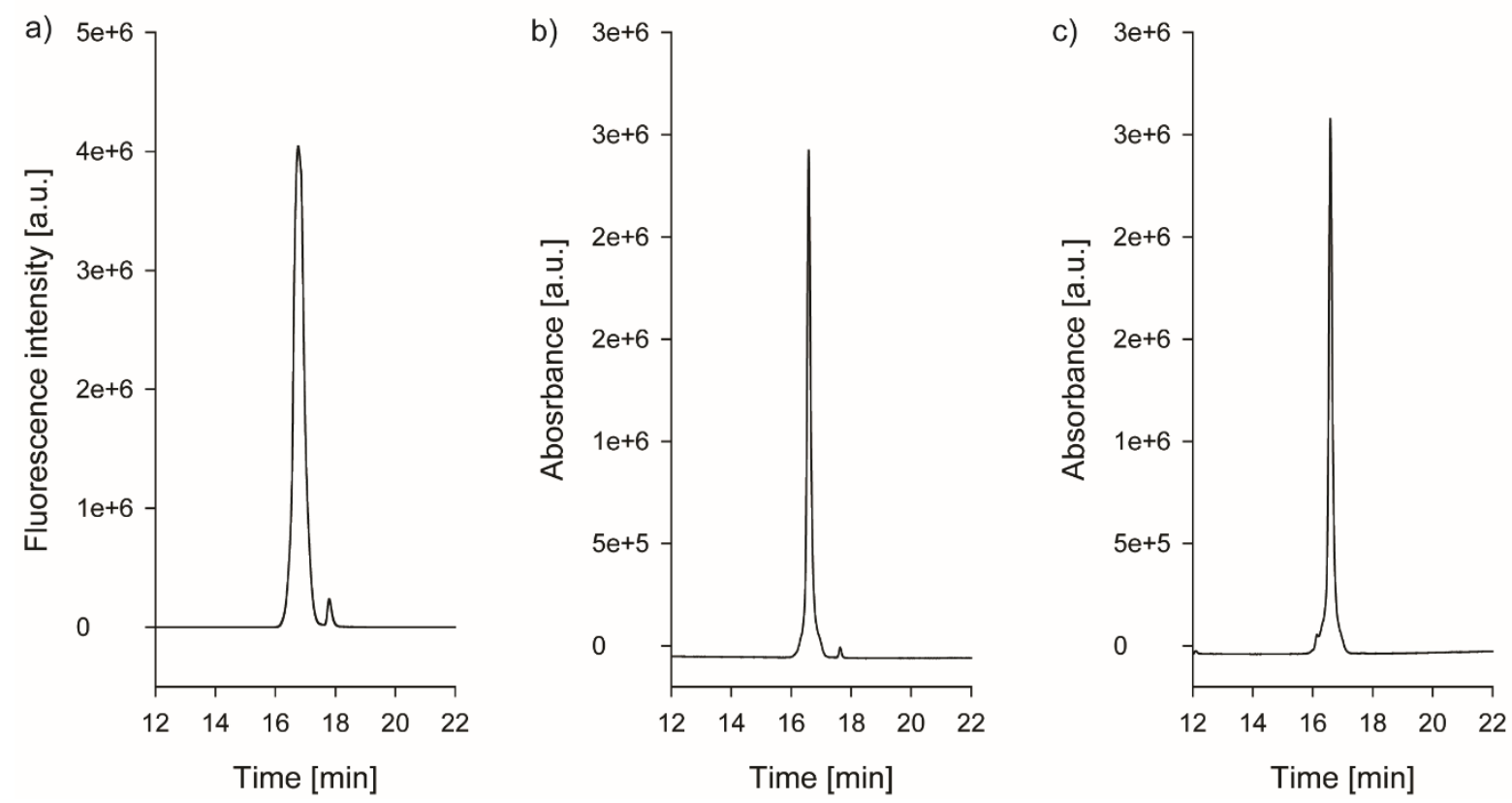

Figure A45: Verification of purity HPLC analysis of PNA-( $\left.\mathrm{PEG}_{10}\right)_{3}-\mathrm{Cy} 5$. a) Fluorescence detection $\left.\left(\lambda_{\mathrm{ex}}=620 \mathrm{~nm}, \lambda_{\mathrm{em}}=665 \mathrm{~nm}\right) \mathrm{b}\right)$ absorbance at $\left.640 \mathrm{~nm} \mathrm{c}\right)$ absorbance at $260 \mathrm{~nm}$. 


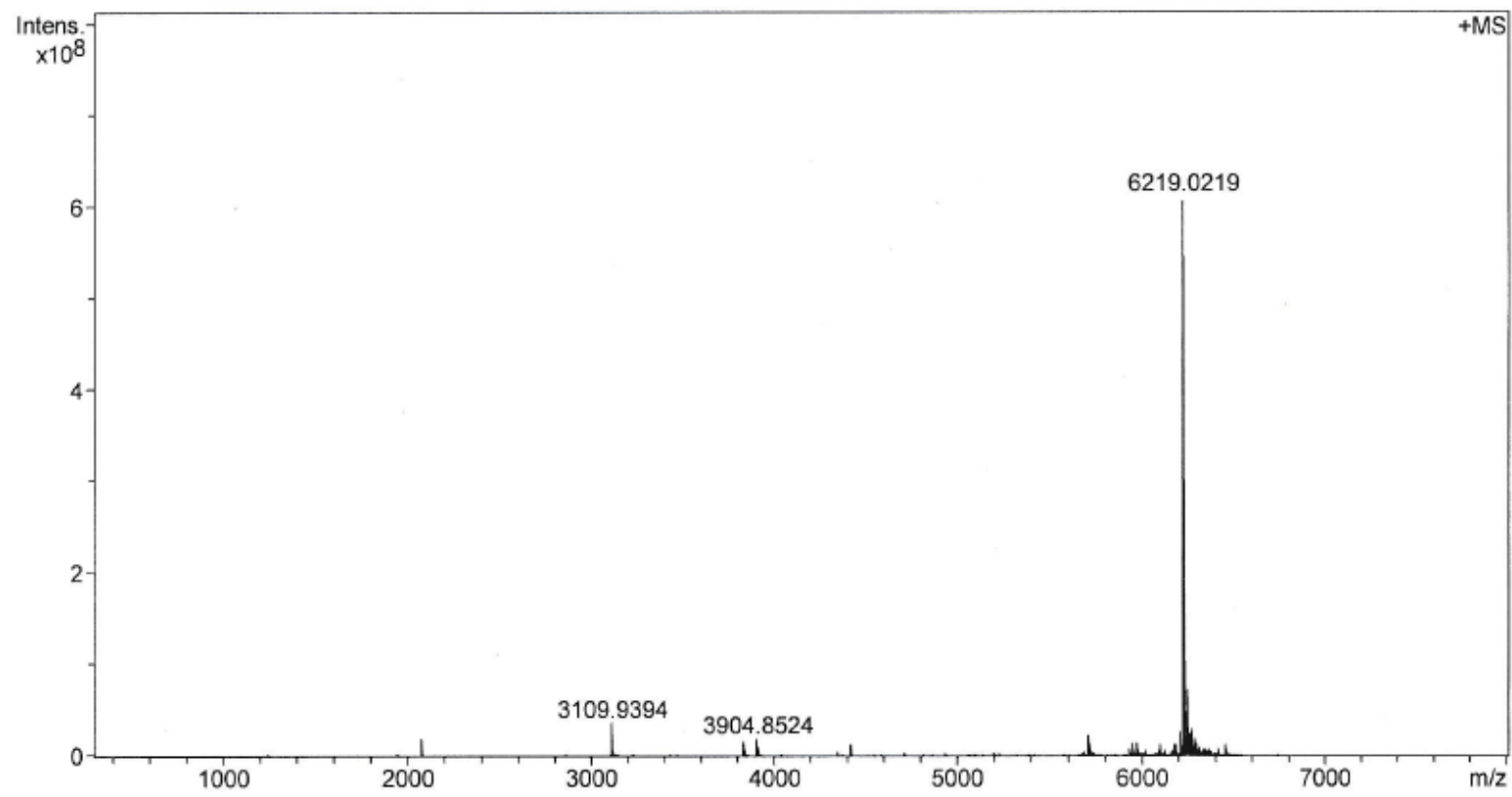

Figure A46: HRMS-MALDI of PNA-(PEG10)3-Cy5.
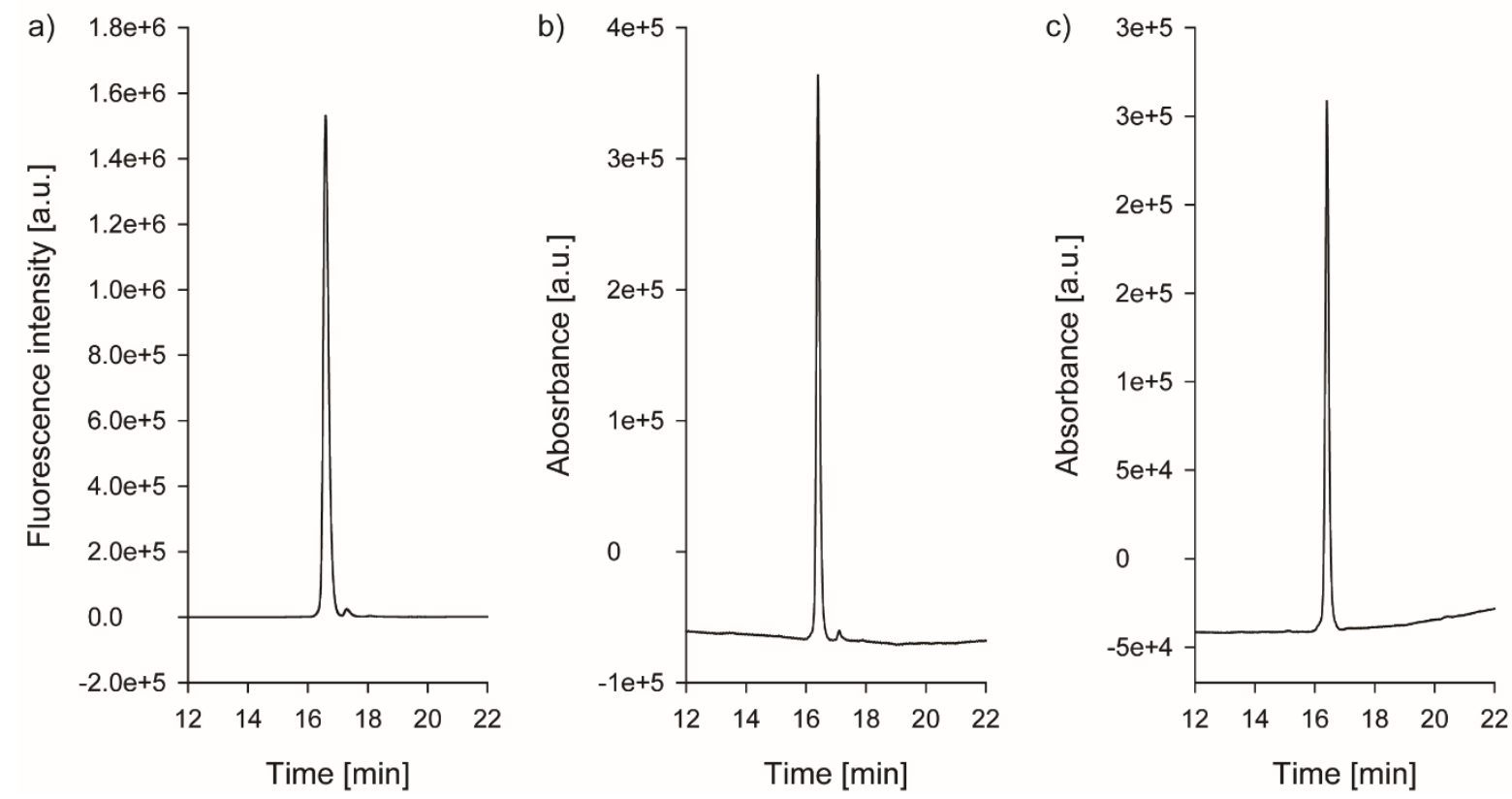

Figure A47: Verification of purity by HPLC analysis of PNA-(PEG 10$)_{4}-\mathrm{Cy} 5$. a) Fluorescence detection $\left.\left(\lambda_{\mathrm{ex}}=620 \mathrm{~nm}, \lambda_{\mathrm{em}}=665 \mathrm{~nm}\right) \mathrm{b}\right)$ absorbance at $\left.640 \mathrm{~nm} \mathrm{c}\right)$ absorbance at $260 \mathrm{~nm}$. 


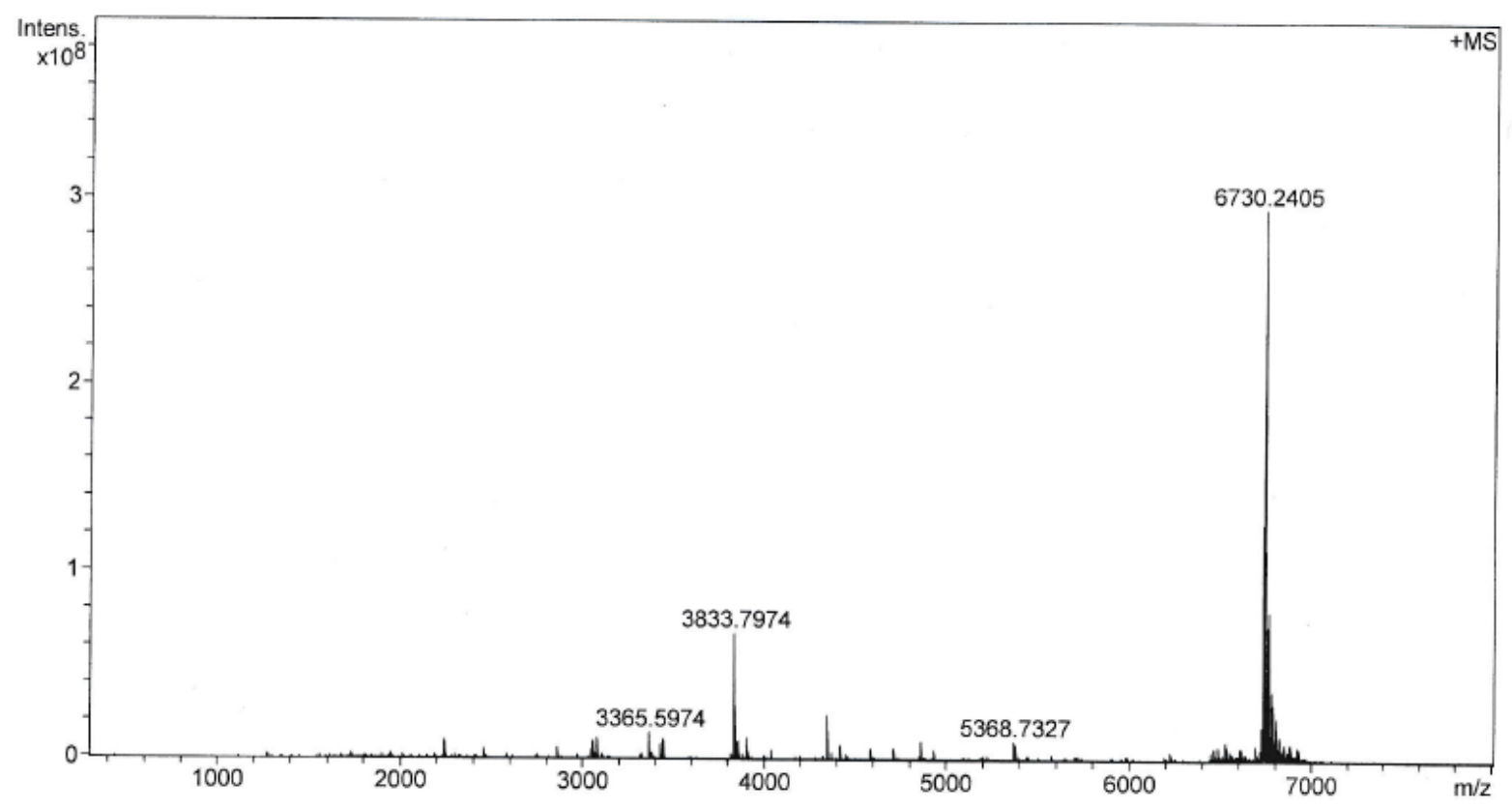

Figure A48: HRMS-MALDI of PNA-(PEG10) $)_{4}-\mathrm{Cy} 5$. 


\section{References}

[1] M. B. Hampton, A. J. Kettle, C. C. Winterbourn, Blood 1998, 92, 3007-3017.

[2] C. Dahlgren, A. Karlsson, J. Immunol. Methods 1999, 232, 3-14.

[3] B. M. Fournier, C. A. Parkos, Mucosal Immunol. 2012, 5, 354-366.

[4] M. Valko, D. Leibfritz, J. Moncol, M. T. D. Cronin, M. Mazur, J. Telser, Int. J. Biochem. Cell Biol. 2007, 39, 44-84.

[5] T. Finkel, J. Cell Biol. 2011, 194, 7-15.

[6] M. P. Murphy, A. Holmgren, N.-G. Larsson, B. Halliwell, C. J. Chang, B. Kalyanaraman, S. G. Rhee, P. J. Thornalley, L. Partridge, D. Gems, et al., Cell Metab. 2011, 13, 361366.

[7] K. M. Holmström, T. Finkel, Nat. Rev. Mol. Cell Biol. 2014, 15, 411-421.

[8] J. Frijhoff, P. G. Winyard, N. Zarkovic, S. S. Davies, R. Stocker, D. Cheng, A. R. Knight, E. L. Taylor, J. Oettrich, T. Ruskovska, et al., Antioxid. Redox Signal. 2015, 23, 114470.

[9] A. I. Casas, V. T.-V. Dao, A. Daiber, G. J. Maghzal, F. Di Lisa, N. Kaludercic, S. Leach, A. Cuadrado, V. Jaquet, T. Seredenina, et al., Antioxid. Redox Signal. 2015, 23, 11711185.

[10] M. C. Martínez, R. Andriantsitohaina, Antioxid. Redox Signal. 2009, 11, 669-702.

[11] K. Krumova, G. Cosa, Chapter 1. Overview of Reactive Oxygen Species, 2016.

[12] B. M. Babior, Curr. Opin. Immunol. 2004, 16, 42-47.

[13] S. Mocellin, V. Bronte, D. Nitti, Med. Res. Rev. 2007, 27, 317-352.

[14] J. P. Kehrer, J. D. Robertson, C. V Smith, in Compr. Toxicol. (Second Ed. (Ed.: A.M. Editor-in-Chief: Charlene), Elsevier, Oxford, 2010, pp. 277-307.

[15] C. C. Winterbourn, Nat. Chem. Biol. 2008, 4, 278-286.

[16] R. Radi, G. Peluffo, M. N. Alvarez, M. Naviliat, A. Cayota, Free Radic. Biol. Med. 2001, 30, 463-488.

[17] J. C. Irvine, R. H. Ritchie, J. L. Favaloro, K. L. Andrews, R. E. Widdop, B. K. KempHarper, Trends Pharmacol. Sci. 2008, 29, 601-608.

[18] C. Nathan, A. Cunningham-Bussel, Nat. Rev. Immunol. 2013, 13, 349-61.

[19] A. R. Cross, A. W. Segal, Biochim. Biophys. Acta 2004, 1657, 1-22.

[20] K. Bedard, K.-H. Krause, Physiol. Rev. 2007, 87, 245-313.

[21] G. J. Maghzal, K.-H. Krause, R. Stocker, V. Jaquet, Free Radic. Biol. Med. 2012, 53, 1903-18.

[22] B. Amulic, C. Cazalet, G. L. Hayes, K. D. Metzler, A. Zychlinsky, Annu. Rev. Immunol. 2012, 30, 459-489. 
[23] E. Kolaczkowska, P. Kubes, Nat. Rev. Immunol. 2013, 13, 159-175.

[24] V. Delgado-Rizo, M. A. Martínez-Guzmán, L. Iñiguez-Gutierrez, A. García-Orozco, A. Alvarado-Navarro, M. Fafutis-Morris, Front. Immunol. 2017, 8, 81.

[25] S. K. Jorch, P. Kubes, Nat. Med. 2017, 23, 279-287.

[26] B. H. Segal, L. Romani, P. Puccetti, Cell. Mol. Life Sci. 2009, 66, 553-558.

[27] E. Song, G. Bala Jaishankar, H. Saleh, W. Jithpratuck, R. Sahni, G. Krishnaswamy, Clin. Mol. Allergy 2011, 10.

[28] O. Wéra, P. Lancellotti, C. Oury, J. Clin. Med. 2016, 5, 118.

[29] C. J. Chang, T. Gunnlaugsson, T. D. James, Chem. Soc. Rev. 2015, 44, 4484-4486.

[30] J. A. Thomas, Chem. Soc. Rev. 2015, 44, 4494-4500.

[31] T. D. Ashton, K. A. Jolliffe, F. M. Pfeffer, Chem. Soc. Rev. 2015, 44, 4547-4595.

[32] Z. Guo, S. Park, J. Yoon, I. Shin, Chem. Soc. Rev. 2014, 43, 16-29.

[33] X. Chen, F. Wang, J. Y. Hyun, T. Wei, J. Qiang, X. Ren, I. Shin, J. Yoon, Chem. Soc. Rev. 2016, 45, 2976-3016.

[34] Z. Lou, P. Li, K. Han, Acc. Chem. Res. 2015, 48, 1358-1368.

[35] X. Li, X. Gao, W. Shi, H. Ma, Chem. Rev. 2014, 114, 590-659.

[36] Y. Tang, D. Lee, J. Wang, G. Li, J. Yu, W. Lin, J. Yoon, Chem. Soc. Rev. 2015, 44, 5003-5015.

[37] M. Ekstrand, M. Gustafsson Trajkovska, J. Perman-Sundelin, P. Fogelstrand, M. Adiels, M. Johansson, L. Mattsson-Hulten, J. Boren, M. Levin, PLoS One 2015, 10, e0130898.

[38] C. Duval, A.-V. Cantero, N. Auge, L. Mabile, J.-C. Thiers, A. Negre-Salvayre, R. Salvayre, Free Radic. Biol. Med. 2003, 35, 1589-1598.

[39] G. Bauer, Anticancer Res. 2014, 34, 1467-82.

[40] K. Kundu, S. F. Knight, N. Willett, S. Lee, W. R. Taylor, N. Murthy, Angew. Chemie Int. Ed. 2009, 48, 299-303.

[41] J. F. Woolley, J. Stanicka, T. G. Cotter, Trends Biochem. Sci. 2013, 38, 556-565.

[42] T. Nagano, T. Yoshimura, Chem. Rev. 2002, 102, 1235-1269.

[43] W. M. Nauseef, Biochim. Biophys. Acta - Gen. Subj. 2014, 1840, 757-767.

[44] T. Nagano, J. Clin. Biochem. Nutr. 2009, 45, 111-124.

[45] A. Gomes, E. Fernandes, J. L. F. C. Lima, J. Fluoresc. 2006, 16, 119-139.

[46] M. Yazdani, Toxicol. Vitr. 2015, 30, 578-582.

[47] K. Debowska, D. Debski, M. Hardy, M. Jakubowska, B. Kalyanaraman, A. Marcinek, R. Michalski, B. Michalowski, O. Ouari, A. Sikora, et al., Pharmacol. Reports 2015, 67, 756-764.

[48] C. C. Winterbourn, Biochim. Biophys. Acta 2014, 1840, 730-8.

[49] X. Chen, X. Tian, I. Shin, J. Yoon, Chem. Soc. rev. 2011, 40, 4783-804. 
[50] H. S. Wang, TrAC - Trends Anal. Chem. 2016, 85, 181-202.

[51] L. Yuan, W. Lin, K. Zheng, L. He, W. Huang, Chem. Soc. Rev. 2013, 42, 622-661.

[52] L. M. Uusitalo, N. Hempel, Int. J. Mol. Sci. 2012, 13, 10660-10679.

[53] O. Adegoke, P. B. C. Forbes, Anal. Chim. Acta 2015, 862, 1-13.

[54] S. Xu, J. Cui, L. Wang, TrAC Trends Anal. Chem. 2016, 80, 149-155.

[55] O. S. Wolfbeis, Chem. Soc. Rev. 2015, 44, 4743-4768.

[56] B. C. Dickinson, D. Srikun, C. J. Chang, Curr. Opin. Chem. Biol. 2010, 14, 50-56.

[57] W. Xu, Z. Zeng, J. H. Jiang, Y. T. Chang, L. Yuan, Angew. Chemie - Int. Ed. 2016, 55, 13658-13699.

[58] X. Zhang, F. Gao, Free Radic. Res. 2015, 49, 374-382.

[59] V. J. Pansare, S. Hejazi, W. J. Faenza, R. K. Prud'homme, Chem. Mater. 2012, 24, 812 827.

[60] G. Grynkiewicz, M. Poenie, R. Y. Tsien, J. Biol. Chem. 1985, 260, 3440-3450.

[61] J. Fan, M. Hu, P. Zhan, X. Peng, Chem. Soc. Rev. 2013, 42, 29-43.

[62] J. Chan, S. C. Dodani, C. J. Chang, Nat. Chem. 2012, 4, DOI 10.1038/NCHEM.1500.

[63] A. J. Meyer, T. P. Dick, Antioxid. Redox Signal. 2010, 13, 621-650.

[64] D. S. Bilan, V. V. Belousov, Antioxid. Redox Signal. 2016, 24, 731-51.

[65] M. Schwarzländer, T. P. Dick, A. J. Meyer, B. Morgan, Antioxid. Redox Signal. 2016, $24,680-712$.

[66] K. A. Lukyanov, V. V. Belousov, Biochim. Biophys. Acta - Gen. Subj. 2014, 1840, 745756.

[67] A. R. Lippert, G. C. Van De Bittner, C. J. Chang, Acc. Chem. Res. 2011, 44, 793-804.

[68] D. Srikun, E. W. Miller, D. W. Domaille, C. J. Chang, J. Am. Chem. Soc. 2008, 130, 4596-4597.

[69] Y. Wen, K. Liu, H. Yang, Y. Li, H. Lan, Y. Liu, X. Zhang, T. Yi, Anal. Chem. 2014, 86, 9970-9976.

[70] Y. Wen, K. Liu, H. Yang, Y. Liu, L. Chen, Z. Liu, C. Huang, T. Yi, Anal. Chem. 2015, 87, 10579-10584.

[71] C. Chung, D. Srikun, C. S. Lim, C. J. Chang, B. R. Cho, Chem. Commun. 2011, 47, 9618-9620.

[72] G. Masanta, C. H. Heo, C. S. Lim, S. K. Bae, B. R. Cho, H. M. Kim, Chem. Commun. 2012, 48, 3518-3520.

[73] F. Du, Y. Min, F. Zeng, C. Yu, S. Wu, Small 2014, 10, 964-972.

[74] G. Wu, F. Zeng, C. Yu, S. Wu, W. Li, J. Mater. Chem. B 2014, 2, 8528-8537.

[75] A. Sikora, J. Zielonka, M. Lopez, J. Joseph, B. Kalyanaraman, Free Radic. Biol. Med. 2009, 47, 1401-1407. 
[76] A. E. Albers, V. S. Okreglak, C. J. Chang, J. Am. Chem. Soc. 2006, 128, 9640-9641.

[77] M. Kumar, N. Kumar, V. Bhalla, P. R. Sharma, Y. Qurishi, Chem. Commun. 2012, 48, 4719-21.

[78] Y. Y. Qian, L. Xue, D. X. Hu, G. P. Li, H. Jiang, Dye. Pigment. 2012, 95, 373-376.

[79] S. W. Lee, H.-W. W. Rhee, Y.-T. T. Chang, J.-I. I. Hong, Chem. - A Eur. J. 2013, 19, 14791-14794.

[80] G. Li, D. Zhu, Q. Liu, L. Xue, H. Jiang, Org. Lett. 2013, 15, 924-927.

[81] B. Zhu, H. Jiang, B. Guo, C. Shao, H. Wu, B. Du, Q. Wei, Sensors Actuators, B Chem. 2013, 186, 681-686.

[82] R. Bortolozzi, S. von Gradowski, H. Ihmels, K. Schäfer, G. Viola, Chem. Commun. 2014, 50, 8242-8245.

[83] M. Xu, J. M. Han, C. Wang, X. Yang, J. Pei, L. Zang, ACS Appl. Mater. Interfaces 2014, $6,8708-8714$.

[84] Y. Lei, C. Xue, S. Zhang, Y. Sha, Luminescence 2016, 31, 660-664.

[85] H. Xiao, P. Li, X. Hu, X. Shi, W. Zhang, B. Tang, Chem. Sci. 2016, 7, 6153-6159.

[86] J. Xu, J. Zhai, Y. Xu, J. Zhu, Y. Qin, D. Jiang, Analyst 2016, 141, 2380-2383.

[87] X. Zhou, L. Lesiak, R. Lai, J. R. Beck, J. Zhao, C. G. Elowsky, H. Li, C. I. Stains, Angew. Chemie - Int. Ed. 2017, 56, 4197-4200.

[88] F. He, Y. Tang, M. Yu, S. Wang, Y. Li, D. Zhu, Adv. Funct. Mater. 2006, 16, 91-94.

[89] F. He, F. Feng, S. Wang, Y. Li, D. Zhu, J. Mater. Chem. 2007, 17, 3702-3707.

[90] D. Srikun, A. E. Albers, C. J. Chang, Chem. Sci. 2011, 2, 1156.

[91] J. Qiao, L. Zhi, Y. Tian, M. Wu, Z. Niu, Chem. Commun. 2015, 51, 3641-3644.

[92] H. N. Kim, M. H. Lee, H. J. Kim, J. S. Kim, J. Yoon, Chem. Soc. Rev. 2008, 37, 14651472 .

[93] L. Yuan, W. Lin, Y. Xie, B. Chen, J. Song, Chem. - A Eur. J. 2012, 18, 2700-2706.

[94] L. Long, D. Zhang, X. Li, J. Zhang, C. Zhang, L. Zhou, Anal. Chim. Acta 2013, 775, $100-105$.

[95] Y.-R. Zhang, X.-P. Chen, Jing-Shao, J.-Y. Zhang, Q. Yuan, J.-Y. Miao, B.-X. Zhao, Chem. Commun. 2014, 50, 14241-14244.

[96] J.-T. Hou, K. Li, J. Yang, K.-K. Yu, Y.-X. Liao, Y.-Z. Ran, Y.-H. Liu, X.-D. Zhou, X.Q. Yu, Chem. Commun. 2015, 51, 6781-6784.

[97] Y.-R. Zhang, Z.-M. Zhao, L. Su, J.-Y. Miao, B.-X. Zhao, RSC Adv. 2016, 6, 1705917063.

[98] H. J. Lee, M. J. Cho, S.-K. Chang, Inorg. Chem. 2015, 54, 8644-8649.

[99] Y. R. Zhang, N. Meng, J. Y. Miao, B. X. Zhao, Chem. - A Eur. J. 2015, 21, 1905819063.

[100] Y.-R. Zhang, Z.-M. Zhao, J.-Y. Miao, B.-X. Zhao, Sensors Actuators B Chem. 2016, 
$229,408-413$.

[101] Y. Liu, Z.-M. Zhao, J.-Y. Miao, B.-X. Zhao, Anal. Chim. Acta 2016, 921, 77-83.

[102] M. Ren, B. Deng, K. Zhou, X. Kong, J.-Y. Wang, G. Xu, W. Lin, J. Mater. Chem. B 2016, 4, 4739-4745.

[103] Z. Zhang, J. Fan, G. Cheng, S. Ghazali, J. Du, X. Peng, Sensors Actuators B Chem. 2017, 246, 293-299.

[104] S.-L. Shen, J.-Y. Ning, X.-F. Zhang, J.-Y. Miao, B.-X. Zhao, Sensors Actuators B Chem. 2017, 244, 907-913.

[105] J. Xu, H. Yuan, C. Qin, L. Zeng, G.-M. Bao, Anal. Chim. Acta 2017, 950, 178-183.

[106] H. Xiao, J. Li, J. Zhao, G. Yin, Y. Quan, J. Wang, R. Wang, J. Mater. Chem. B 2015, 3, $1633-1638$.

[107] W. Lin, L. Long, B. Chen, W. Tan, Chem. - A Eur. J. 2009, 15, 2305-2309.

[108] L. Yuan, W. Lin, J. Song, Y. Yang, Chem. Commun. 2011, 47, 12691.

[109] S. Goswami, A. Manna, S. Paul, C. K. Quah, H.-K. Fun, Chem. Commun. 2013, 49, 11656-11658.

[110] Z. Lou, P. Li, P. Song, K. Han, Analyst 2013, 138, 6291-5.

[111] J. Park, H. Kim, Y. Choi, Y. Kim, Analyst 2013, 138, 3368-3371.

[112] F. Ma, M. Sun, K. Zhang, Y. Zhang, H. Zhu, L. Wu, D. Huang, S. Wang, S. A. Hilderbrand, S. Y. Lee, et al., RSC Adv. 2014, 4, 59961-59964.

[113] J. Fan, H. Mu, H. Zhu, J. Du, N. Jiang, J. Wang, X. Peng, Ind. Eng. Chem. Res. 2015, $54,8842-8846$.

[114] S. Goswami, K. Aich, S. Das, B. Pakhira, K. Ghoshal, C. K. Quah, M. Bhattacharyya, H. K. Fun, S. Sarkar, Chem. - An Asian J. 2015, 10, 694-700.

[115] J. Li, C. Yin, F. Huo, K. Xiong, J. Chao, Y. Zhang, Sensors Actuators, B Chem. 2016, $231,547-551$.

[116] W. Shu, P. Jia, X. Chen, X. Li, Y. Huo, F. Liu, Z. Wang, C. Liu, B. Zhu, L. Yan, et al., Rsc Adv. 2016, 6, 64315-64322.

[117] L. L. Wang, Y. Hu, Y. Qu, J. L. Xu, J. Cao, Dye. Pigment. 2016, 128, 54-59.

[118] H. Chen, H. Shang, Y. Liu, R. Guo, W. Lin, Adv. Funct. Mater. 2016, 26, 8128-8136.

[119] X. Wang, L. Zhou, F. Qiang, F. Wang, R. Wang, C. Zhao, Anal. Chim. Acta 2016, 911, $114-120$.

[120] J. Kang, F. Huo, Y. Yue, Y. Wen, J. Chao, Y. Zhang, C. Yin, Dye. Pigment. 2017, 136, $852-858$.

[121] C. Chang, F. Wang, J. Qiang, Z. Zhang, Y. Chen, W. Zhang, Y. Wang, X. Chen, Sensors Actuators B Chem. 2017, 243, 22-28.

[122] G. Chen, F. Song, J. Wang, Z. Yang, S. Sun, J. Fan, X. Qiang, X. Wang, B. Dou, Chem. Commun. 2012, 48, 2949-2951. 
[123] X. Wu, Z. Li, L. Yang, J. Han, S. Han, Chem. Sci. 2013, 4, 460-467.

[124] F. C. Fang, Nat. Rev. Microbiol. 2004, 2, 820-832.

[125] N. Soh, K. Makihara, E. Sakoda, T. Imato, Chem. Commun. 2004, 496-497.

[126] D. Oushiki, H. Kojima, T. Terai, M. Arita, K. Hanaoka, Y. Urano, T. Nagano, J. Am. Chem. Soc. 2010, 132, 2795-2801.

[127] L. Yuan, W. Lin, J. Song, Chem. Commun. 2010, 46, 7930.

[128] M. Zhuang, C. Ding, A. Zhu, Y. Tian, Anal. Chem. 2014, 86, 1829-1836.

[129] L. Meng, Y. Wu, T. Yi, Chem. Commun. 2014, 50, 4843-4845.

[130] M. King, R. Kopelman, Sensors Actuators, B Chem. 2003, 90, 76-81.

[131] G. M. Ganea, P. E. Kolic, B. El-Zahab, I. M. Warner, Anal. Chem. 2011, 83, 2576-2581.

[132] J. Wang, X. Xu, Y. Zhao, C. Zheng, L. Li, J. Mater. Chem. 2011, 21, 18696.

[133] S. Liu, J. Zhao, K. Zhang, L. Yang, M. Sun, H. Yu, Y. Yan, Y. Zhang, L.-J. Wu, S. Wang, Analyst 2016, 141, 2296-2302.

[134] Q. Zhao, R. Zhang, D. Ye, S. Zhang, H. Chen, J. Kong, ACS Appl. Mater. Interfaces 2017, 9, 2052-2058.

[135] M. Mittal, M. R. Siddiqui, K. Tran, S. P. Reddy, A. B. Malik, Antioxid. Redox Signal. 2014, 20, 1126-67.

[136] B. Kalyanaraman, B. P. Dranka, M. Hardy, R. Michalski, J. Zielonka, Biochim. Biophys. Acta - Gen. Subj. 2014, 1840, 739-744.

[137] A. Gomes, E. Fernandes, J. L. F. C. Lima, J. Biochem. Biophys. Methods 2005, 65, 4580 .

[138] X. Gao, C. Ding, A. Zhu, Y. Tian, Anal. Chem. 2014, 86, 7071-7078.

[139] H. Huang, F. Dong, Y. Tian, Anal. Chem. 2016, 88, 12294-12302.

[140] D. Andina, D. Brambilla, N. Munzinger, J. Frei, C. Zivko, J.-C. Leroux, P. Luciani, Chem. - A Eur. J. 2017, 23, 4765-4769.

[141] D. P. Murale, H. Kim, W. S. Choi, D. G. Churchill, Org. Lett. 2013, 15, 3946-3949.

[142] X. Gao, G. Feng, P. N. Manghnani, F. Hu, N. Jiang, J. Liu, B. Liu, J. Z. Sun, B. Z. Tang, Chem. Commun. 2017, 53, 1653-1656.

[143] Y. Zhou, J. Ding, T. Liang, E. S. Abdel-Halim, L. Jiang, J. J. Zhu, ACS Appl. Mater. Interfaces 2016, 8, 6423-6430.

[144] L. E. McQuade, S. J. Lippard, Curr. Opin. Chem. Biol. 2010, 14, 43-49.

[145] M. H. Lim, S. J. Lippard, Acc. Chem. Res. 2007, 40, 41-51.

[146] L. Yuan, W. Lin, Y. Xie, B. Chen, J. Song, Chem. Commun. 2011, 47, 9372.

[147] H. Yu, L. Jin, Y. Dai, H. Li, Y. Xiao, New J. Chem. 2013, 37, 1688.

[148] C. Yu, Y. Wu, F. Zeng, S. Wu, J. Mater. Chem. B 2013, 1, 4152-4159.

[149] R. Radi, J. Biol. Chem. 2013, 288, 26464-26472. 
[150] J. Zielonka, A. Sikora, M. Hardy, J. Joseph, B. P. Dranka, B. Kalyanaraman, Chem. Res. Toxicol. 2012, 25, 1793-1799.

[151] Y. Zhou, W. B. Pei, C. Y. Wang, J. X. Zhu, J. S. Wu, Q. Y. Yan, L. Huang, W. Huang, C. Yao, J. S. C. Loo, et al., Small 2014, 10, 3560-3567.

[152] S. Palanisamy, P.-Y. Wu, S.-C. Wu, Y.-J. Chen, S.-C. Tzou, C.-H. Wang, C.-Y. Chen, Y.-M. Wang, Biosens. Bioelectron. 2017, 91, 849-856.

[153] Y.-X. Liao, Z.-X. Yang, K. Li, X.-Q. Yu, Chem. Lett. 2016, 45, 691-693.

[154] Z. Li, S.-H. Yan, C. Chen, Z.-R. Geng, J.-Y. Chang, C.-X. Chen, B.-H. Huang, Z.-L. Wang, Biosens. Bioelectron. 2017, 90, 75-82.

[155] N. Soh, T. Imato, K. Kawamura, M. Maeda, Y. Katayama, Chem. Commun. 2002, 327, 2650-2651.

[156] A. K. Mahapatra, S. S. Ali, K. Maiti, S. Mondal, R. Maji, S. Manna, S. K. Manna, M. R. Uddin, S. Mandal, RSC Adv. 2016, 6, 113219-113227.

[157] D. Cheng, Y. Pan, L. Wang, Z. Zeng, L. Yuan, X. Zhang, Y.-T. Chang, J. Am. Chem. Soc. 2017, 139, 285-292.

[158] B. Wang, F. Yu, P. Li, X. Sun, K. Han, Dye. Pigment. 2013, 96, 383-390.

[159] J. Zhou, Y. Li, J. Shen, Q. Li, R. Wang, Y. Xu, X. Qian, Rsc Adv. 2014, 4, 51589-51592.

[160] X. Zhou, Y. Kwon, G. Kim, J. H. Ryu, J. Yoon, Biosens. Bioelectron. 2015, 64, 285291.

[161] X. Jia, Q. Chen, Y. Yang, Y. Tang, R. Wang, Y. Xu, W. Zhu, X. Qian, J. Am. Chem. Soc. 2016, 138, 10778-10781.

[162] J. Tian, H. Chen, L. Zhuo, Y. Xie, N. Li, B. Tang, Chem. - A Eur. J. 2011, 17, 66266634.

[163] K. M. Miranda, N. Paolocci, T. Katori, D. D. Thomas, E. Ford, M. D. Bartberger, M. G. Espey, D. A. Kass, M. Feelisch, J. M. Fukuto, et al., Proc. Natl. Acad. Sci. U. S. A. 2003, 100, 9196-201.

[164] C. Liu, H. Wu, Z. Wang, C. Shao, B. Zhu, X. Zhang, Chem. Commun. 2014, 50, 60136016.

[165] H. T. Zhang, R. C. Liu, Y. Tan, W. H. Xie, H. P. Lei, H.-Y. Y. Cheung, H. Y. Sun, ACS Appl. Mater. Interfaces 2015, 7, 5438-5443.

[166] H.-M. Lv, Y. Chen, J. Lei, C. Au, S.-F. Yin, S. Imbrogno, C. Capria, B. Tota, F. B. Jensen, J. A. Reisz, et al., Anal. Methods 2015, 7, 3883-3887.

[167] X. Zhu, M. Xiong, H. Liu, G. Mao, L. Zhou, J. Zhang, X. Hu, X.-B. Zhang, W. Tan, F. Doctorovich, et al., Chem. Commun. 2016, 52, 733-736.

[168] A. J. Shuhendler, K. Pu, L. Cui, J. P. Uetrecht, J. Rao, Nat. Biotechnol. 2014, 32, 37380.

[169] K. Zheng, H. Chen, S. Fang, Y. Wang, Sensors Actuators B Chem. 2016, 233, 193-198.

[170] F. Liu, T. Wu, J. Cao, H. Zhang, M. Hu, S. Sun, F. Song, J. Fan, J. Wang, X. Peng, Analyst 2013, 138, 775-778. 
[171] T. Chen, Y. Hu, Y. Cen, X. Chu, Y. Lu, J. Am. Chem. Soc. 2013, 135, 11595-11602.

[172] E. Ju, Z. Liu, Y. Du, Y. Tao, J. Ren, X. Qu, ACS Nano 2014, 8, 6014-6023.

[173] F. Liu, T. Bing, D. Shangguan, M. Zhao, N. Shao, Anal. Chem. 2016, 88, 10631-10638.

[174] J. P. Giraldo, M. P. Landry, S. Y. Kwak, R. M. Jain, M. H. Wong, N. M. Iverson, M. Ben-Naim, M. S. Strano, Small 2015, 11, 3973-3984.

[175] Q. Zhang, Z. Zhu, Y. Zheng, J. Cheng, N. Zhang, Y.-T. Long, J. Zheng, X. Qian, Y. Yang, J. Am. Chem. Soc. 2012, 134, 18479-18482.

[176] J. L. Kolanowski, A. Kaur, E. J. New, Antioxid. Redox Signal. 2016, 24, 713-730.

[177] A. Kaur, J. L. Kolanowski, E. J. New, Angew. Chemie - Int. Ed. 2016, 55, 1602-1613.

[178] A. Kaur, M. A. Haghighatbin, C. F. Hogan, E. J. New, Chem. Commun. 2015, 51, 1051010513.

[179] A. Kaur, K. Jankowska, C. Pilgrim, S. T. Fraser, E. J. New, Antioxid. Redox Signal. 2016, 24, 667-679.

[180] K. Kundu, S. F. Knight, S. Lee, W. R. Taylor, N. Murthy, Angew. Chemie - Int. Ed. 2010, 49, 6134-6138.

[181] M. Levitus, S. Ranjit, Q. Rev. Biophys. 2011, 44, 123-151.

[182] P. W. Lin, L. E. S. Myers, L. Ray, S. C. Song, T. R. Nasr, A. J. Berardinelli, K. Kundu, N. Murthy, J. M. Hansen, A. S. Neish, Free Radic. Biol. Med. 2009, 47, 1205-1211.

[183] S. Selvam, K. Kundu, K. L. Templeman, N. Murthy, A. J. García, Biomaterials 2011, $32,7785-7792$.

[184] N. J. Willett, K. Kundu, S. F. Knight, S. Dikalov, N. Murthy, W. R. Taylor, Antioxid. Redox Signal. 2011, 15, 1369-78.

[185] P. Goodson, A. Kumar, L. Jain, K. Kundu, N. Murthy, M. Koval, M. N. Helms, Am J Physiol Lung Cell Mol Physiol 2012, 302, L410-9.

[186] L. Xie, A. S. P. Lin, K. Kundu, M. E. Levenston, N. Murthy, R. E. Guldberg, Arthritis Rheum. 2012, 64, 1899-1908.

[187] K. Schoknecht, O. Prager, U. Vazana, L. Kamintsky, D. Harhausen, M. Zille, L. Figge, Y. Chassidim, E. Schellenberger, R. Kovács, et al., J. Cereb. Blood Flow Metab. 2014, $34,1791-801$.

[188] M. C. Prunty, M. H. Aung, A. M. Hanif, R. S. Allen, M. A. Chrenek, J. H. Boatright, P. M. Thule, K. Kundu, N. Murthy, M. T. Pardue, Invest. Ophthalmol. Vis. Sci. 2015, 56, 5862-70.

[189] S. Suri, S. M. Lehman, S. Selvam, K. Reddie, S. Maity, N. Murthy, A. J. García, J. Biomed. Mater. Res. A 2015, 103, 76-83.

[190] S. Magalotti, T. P. Gustafson, Q. Cao, D. R. Abendschein, R. A. Pierce, M. Y. Berezin, W. J. Akers, Mol Imaging Biol 2013, 15, 423-430.

[191] J. Y. Kim, W. Il Choi, Y. H. Kim, G. Tae, J. Control. Release 2011, 156, 398-405.

[192] M. V Kvach, A. V Ustinov, I. A. Stepanova, A. D. Malakhov, M. V Skorobogatyi, V. V Shmanai, V. A. Korshun, European J. Org. Chem. 2008, 2107-2117. 
[193] K. U. Ingold, T. Paul, M. J. Young, J. Am. Chem. Soc. 1997, 7863, 12364-12365.

[194] K. G. Konya, T. Paul, S. Lin, J. Lusztyk, K. U. Ingold, J. Am. Chem. Soc. 2000, 122, 7518-7527.

[195] L. Brülisauer, G. Valentino, S. Morinaga, K. Cam, J. Thostrup Bukrinski, M. A. Gauthier, J.-C. Leroux, J. Thostrup Bukrinski, M. A. Gauthier, J.-C. Leroux, Angew. Chemie - Int. Ed. 2014, 53, 8392-6.

[196] Y. Chen, W. G. Junger, Methods Mol. Biol. 2012, 844, 115-124.

[197] L. M. Henderson, J. B. Chappell, Eur. J. Biochem. 1993, 217, 973-980.

[198] W. Li, P. Zhan, E. De Clercq, H. Lou, X. Liu, Prog. Polym. Sci. 2013, 38, 421-444.

[199] M. A. Hink, N. V. Visser, J. W. Borst, A. van Hoek, A. J. W. G. Vissner, J. Fluoresc. 2003, 13, 185-188.

[200] J. Karolin, L. B.-A. Johansson, L. Strandberg, T. Ny, J. Am. Chem. Soc. 1994, 116, 78017806.

[201] J. R. Albani, Principles and Applications of Fluorescence Spectroscopy, Blackwell Science, 2007.

[202] J. M. Slauch, Mol. Microbiol. 2011, 80, 580-583.

[203] P. Wittung, P. E. Nielsen, O. Buchardt, M. Egholm, B. Norden, Nature 1994, 368, 561563.

[204] A. Sen, P. E. Nielsen, Nucleic Acids Res. 2007, 35, 3367-3374.

[205] B. Ndeboko, G. J. Lemamy, P. E. Nielsen, L. Cova, Int. J. Mol. Sci. 2015, 16, 2823028241.

[206] J. P. Vernille, L. C. Kovell, J. W. Schneider, Bioconjug. Chem. 2004, 15, 1314-21.

[207] P. E. Nielsen, M. Egholm, R. H. Berg, O. Buchardt, Science 1991, 254, 1497-1500.

[208] H. Stender, B. Williams, J. Coull, in Pept. Nucleic Acids, 2014, pp. 167-178.

[209] B. Juskowiak, Anal. Bioanal. Chem. 2011, 399, 3157-76.

[210] J.-M. Nam, C. S. Thaxton, C. A. Mirkin, Science 2003, 301, 1884-6.

[211] H. D. Hill, C. A. Mirkin, Nat. Protoc. 2006, 1, 324-336.

[212] F. P. Schwarz, S. Robinson, J. M. Butler, Nucleic Acids Res. 1999, 27, 4792-4800.

[213] H. Wan, D. Liu, X. Yu, H. Sun, Y. Li, Food Chem. 2015, 175, 601-608.

[214] J. M. Berg, J. L. Tymoczko, L. Stryer, in Stryer Biochem., Springer Berlin Heidelberg, Berlin, Heidelberg, 2013, pp. 25-65.

[215] B. Volcheck, K. Xing, T. Urlacher, K. Kundu, N. Padhye, "Hydrocyanine Based Probes for Imaging Highly Reactive Oxygen Species,” 2012.

[216] H. v. Berlepsch, C. Böttcher, J. Phys. Chem. B 2015, 119, 11900-11909.

[217] R. Chattaraj, P. Mohan, C. M. Livingston, J. D. Besmer, K. Kumar, A. P. Goodwin, ACS Appl. Mater. Interfaces 2016, 8, 802-808. 


\section{List of Abbreviations}

\begin{tabular}{|c|c|}
\hline AAPH & 2,2-azobis(2-methyl-propionamidine) dihydrochloride \\
\hline $\mathrm{Ac}_{2} \mathrm{O}$ & acetic anhydride \\
\hline $\mathrm{ACN}$ & acetonitrile \\
\hline $\mathrm{AcOH}$ & acetic acid \\
\hline APAP & analgesic and anti-pyretic acetaminophen \\
\hline AS & Angeli's salt, sodium $\alpha$-oxyhyponitrite \\
\hline Boc & butyloxycarbonyl \\
\hline BODIPY & boron-dipyrromethene \\
\hline BSA & bovine serum albumin \\
\hline $\mathrm{CCCP}$ & carbonyl cyanide $m$-chlorophenylhydrazone \\
\hline CGD & chronic granulomatous disease \\
\hline $\mathrm{CHCl}_{3}$ & chloroform \\
\hline CONER & coumarin-neutral red nanoparticle \\
\hline cpYFP & circular permuted yellow fluorescent protein \\
\hline DCFHDA & $2^{\prime}, 7^{\prime}$-dichlorofluorescein diacetate \\
\hline DCM & dichloromethane \\
\hline DHE & dihydroethidine \\
\hline DHR & dihydrorhodamine 123 \\
\hline DIPEA & $N, N$-diisopropylethylamine \\
\hline DMEM & Dulbecco's Modified Eagle Medium \\
\hline DMF & dimethylformamide \\
\hline DMSO & dimethyl sulfoxide \\
\hline DNA & deoxyribonucleic acid \\
\hline DOX & doxorubicin \\
\hline EDTA & ethylenediaminetetraacetic acid \\
\hline EGF & epidermal growth factor \\
\hline $\mathrm{Et}_{2} \mathrm{O}$ & diethylether \\
\hline
\end{tabular}




\begin{tabular}{|c|c|}
\hline EtOAc & ethylacetate \\
\hline FACS & fluorescence-activated cell sorting \\
\hline FAD & flavin adenine dinucleotide \\
\hline FAM & 6-carboxyfluorescein \\
\hline FBS & fetal bovine serum \\
\hline $\mathrm{FeSO}_{4}$ & iron(II) sulfate \\
\hline FITC & fluorescein \\
\hline Fmoc & fluorenylmethoxycarbonyl \\
\hline FRET & Förster resonance energy transfer \\
\hline GFP & green fluorescent protein \\
\hline GI & gastro intestinal \\
\hline GSH & glutathione \\
\hline $\mathrm{H}_{2} \mathrm{O}_{2}$ & hydrogen peroxide \\
\hline $\mathrm{H}_{2} \mathrm{O}$ & water \\
\hline HATU & $\begin{array}{l}\text { 1-[bis(dimethylamino)methylene]-1H-1,2,3-triazolo[4,5-b]pyridinium 3- } \\
\text { oxide hexafluorophosphate }\end{array}$ \\
\hline $\mathrm{HE}$ & hydroethidine \\
\hline HEPES & 4-(2-hydroxyethyl)-1-piperazineethanesulfonic acid \\
\hline HBSS & Hank's balanced salt solution \\
\hline $\mathrm{HCl}$ & hydrochloric acid \\
\hline HFIP & hexafluoro-2-propanol \\
\hline $\mathrm{HNO}$ & nitroxyl \\
\hline $\mathrm{HPF}$ & hydroxyphenyl fluorescein \\
\hline IBD & inflammatory bowel disease \\
\hline $\mathrm{IFN} \gamma$ & interferon $\gamma$ \\
\hline${ }^{1} \mathrm{O}_{2}$ & singlet oxygen \\
\hline $\mathrm{HO}^{\circ}$ & hydroxyl radical \\
\hline $\mathrm{HOCl}$ & hypochlorous acid \\
\hline KI & potassium iodide \\
\hline
\end{tabular}


LPS lipopolysaccharides

MeI methyliodide

$\mathrm{MeOH} \quad$ methanol

$\mathrm{Na}_{2} \mathrm{SO}_{4} \quad$ sodium sulfate

$\mathrm{NaBH}_{4} \quad$ sodium borohydride

$\mathrm{NaCl} \quad$ sodium chloride

NADPH nicotinamide adenine dinucleotide phosphate

NET neutrophil extracellular trap

NIR near infrared

NO• nitric oxide

$\mathrm{NO}_{2} \quad$ nitrogen dioxide

NOX subunit of NADPH oxidase

NP nanoparticle

$\mathrm{O}_{2}{ }^{-} \quad$ superoxide

$\mathrm{O}_{3} \quad$ ozone

$\mathrm{ONOO}^{-} \quad$ peroxynitrite

PAGE polyacrylamide gel electrophoresis

PBS phosphate buffered saline

PEBBLE photonic explorer for bioanalysis with biologically localized embedding

PEG poly(ethylene glycol)

PGA poly(glutamic acid)

PMA phorbol-12-myristate-13-acetate

PNA peptide nucleic acid

py pyridine

QD quantum dot

roGFP redox-sensitive green fluorescent protein

ROS reactive oxygen species

SIN-1 3-morpholino-sydnonimine

SOD superoxide dismutase 
SOTS-1 di-(4-carboxybenzyl) hyponitrite

${ }^{t} \mathrm{Bu} \quad$ tert-butyl

TAMRA 5-carboxytetramethylrhodamine

TEER transepithelial resistance

TFA trifluoroacetic acid

Tm tunicamycin

TP two-photon

TPP triphenyl phospine

TRIS tris(hydroxymethyl)aminomethane 


\title{
Curriculum Vitae
}

\author{
Diana Laura Romana Andina
}

Born on September $27^{\text {th }}, 1988$

Citizen of Croglio (TI)

diana.andina@gmx.ch

\section{Education}

06/2014 - 05/2017 Doctoral Studies, ETH Zurich, Switzerland

Ph.D. in Drug Formulation \& Delivery, Institute of Pharmaceutical Sciences, Department of Chemistry and Applied Biosciences Ph.D. thesis title: "Development of a Modular Ratiometric Fluorescent Probe for the Detection of Extracellular Superoxide" Advisors: Prof. Dr. Paola Luciani and Prof. Dr. Jean-Christophe Leroux

09/2011 - 12/2012 Master of Science, Chemistry, ETH Zurich, Switzerland Master's thesis title: "Synthesis of IP5-polymer analogues targeting toxins A and B of C. Difficile"

Advisors: Prof. Dr. Bastien Castagner and Prof. Dr. JeanChristophe Leroux

09/2008 - 09/2011 Bachelor of Science, Chemistry, ETH Zurich, Switzerland

08/2001 - 07/2007 Matura, Kantonsschule Zug, Zug, Switzerland 


\section{Professional Experiences}

06/2014 - 05/2017 Ph.D. research and teaching assistant, ETH Zurich, Switzerland Drug Formulation \& Delivery, Institute of Pharmaceutical Sciences, Department of Chemistry and Applied Biosciences

Teaching: Tutor in the lab course "Allgemeine Chemie für Studierende der Pharmazeutischen Wissenschaften und Biologie“ and research project supervision of several master students

01/2013 - 04/2013 Intern, Staumann AG, Basel, Switzerland

Design and Optimization of a Formulation for Dental Implants

09/2012 - 12/2012 Master's thesis research project, ETH Zurich, Switzerland

Drug Formulation \& Delivery, Institute of Pharmaceutical Sciences, Department of Chemistry and Applied Biosciences

Title: "Synthesis of IP5-polymer analogues targeting toxins A and B of C. Difficile"

04/2012 - 05/2012 Intern, ETH Zurich, Switzerland

Pharmaceutical Chemistry, Institute of Pharmaceutical Sciences Synthesis of oligonucleotide modification

02/2012 - 04/2012 Research Project II, ETH Zurich, Switzerland Synthesis \& Chemical Biology, Laboratory of Organic Chemistry Title: "Towards the synthesis of new N-terminal oxazetidine for ketoacid-hydroxylamine ligation"

02/2011 - 05/2011 Research Project I, ETH Zurich, Switzerland (part-time) Drug Formulation \& Delivery, Institute of Pharmaceutical Sciences, Department of Chemistry and Applied Biosciences Title: "Synthesis of small molecule trigger of $C$. difficile toxin autoprocessing"

$01 / 2011$

Teaching Assistant, ETH Zurich, Switzerland Basic Laboratory Course 


\section{Scientific Contributions}

\section{Publications}

Andina D., Leroux J.-C. \& Luciani P. Ratiometric Fluorescent Probe to Detect Reactive Oxygen Species. Chem. - A Eur. J. (2017) doi:10.1002/chem.201702458.

Gong Y., Andina D., Nahar S., Leroux J.-C., Gauthier M. A. Releasable and Traceless PEGylation of Arginine-rich Antimicrobial Peptides. Chem. Sci. 8, 4082-4086 (2017).

Huang X., Andina D., Ge J., Labarre A., Leroux J.-C., Castagenr B. Characterization of calcium phosphate nanoparticles based on a PEGylated chelator for gene delivery. ACS Appl. Mater. Interfaces 9, 10435-10445 (2017).

Andina D., Brambilla D., Munzinger N., Frei J., Zivko C., Leroux J.-C. \& Luciani P. Development of a Modular Ratiometric Fluorescent Probe for the Detection of Extracellular Superoxide. Chem. - A Eur. J. 23, 4765-4769 (2017).

Proulx S. T., Ma Q., Andina D., Leroux, J.-C. \& Detmar M. Quantitative measurement of lymphatic function in mice by noninvasive near-infrared imaging of a peripheral vein. JCI Insight 2, e90861 (2017).

\section{Oral Presentations}

Andina D., Leroux J.-C., Luciani P., A Ratiometric Fluorescent Probe for the Specific Detection of Neutrophil-Induced Reactive Oxygen Species. Doktorandentag Institute of Pharmaceutical Sciences, ETH Zurich, Switzerland, February 17, 2016.

Andina D., Brambilla, D., Zivko C., Leroux J.-C., Luciani P. Design of a Ratiometric Fluorescent Probe Library for Specific Detection of Reactive Oxygen Species in Inflamed Intestine, WMIC 2015, Honolulu, Hawaii, September 2-5, 2015.

Andina D., Leroux J.-C., Luciani P., Fluorescent Probes for Specific Detection of Reactive Oxygen Species in Inflamed Intestine, Zurich-Geneva-Basel Joint Seminar Series on Drug Formulation \& Delivery, Basel, Switzerland, January 23-24, 2015. 


\section{Poster presentations}

Andina D., Brambilla D., Leroux J.-C., Luciani P.; Fluorescent probes for detection of reactive oxygen species in inflamed intestine; Materials and Processes Graduate Symposium, ETH Zurich, Switzerland, May 12, 2014.

Andina D.; Brambilla D., Leroux J.-C., Luciani P.; Contrast agents for imaging intra- and extracellular reactive oxygen species in inflamed intestine; International Conference and Workshop on Biological Barriers 2014, Saarbrücken, Germany, February 16-21, 2014. 


\section{Acknowledgements}

I would like to sincerely thank Prof. Dr. Jean-Christophe Leroux for giving me the opportunity to work in his group and offering me such an excellent environment for research. I am thankful for his scientific guidance during the good and difficult moments of this thesis and truly appreciate his faith in me.

I would also like to express my deepest gratefulness to Prof. Dr. Paola Luciani, my cosupervisor and co-examiner, for her consistent guidance and her unconfined support in and outside the laboratory. She was always available to discuss results and problems and encouraged me to go on.

My thanks go to Prof. Dr. Pablo Rivera Fuentes for being one of my co-examiners of this thesis. I gratefully acknowledge Dr. Davide Brambilla for his work on hydrocyanines and the help throughout the thesis.

Many thanks go to Dr. Jong Ah Kim for proofreading the thesis and her friendship.

I would like to thank Noah Munzinger, Dr. Patrick Wilhelm and Jasmine Egli for their scientific support in peptide synthesis and purification.

Special thanks also go to Dr. Erica Russo for teaching me how to isolate neutrophils from fresh blood samples and for her support with the neutrophils experiments.

Furthermore, I would like to thank Rolf Häfliger from the mass spectrometry service of the Institute of Organic Chemistry for the countless MALDI-MS measurements.

I am also thankful to the Scientific Center for Optical and Electron Microscopy (ScopeM) for their assistance in histological sample preparation.

I am grateful to my students, Cristina Zivko, Jasmine Frei and Ryan Dragoman for their enthusiasm in the laboratory and their hard work during their projects. During our discussions, I learnt a lot about how to supervise a project.

Big thanks go to the current and former members of the Drug Formulation \& Delivery group for creating such a welcoming atmosphere. Without their support and motivation, the time in the laboratory but also time spent together outside would not have been as memorable. Some special thanks go to Virginie Rusca for her kindness, her goodwill and friendship. I would like 
to thank Dr. Athanasia Dasargyri, Dr. Jessica Schulz and Britta Hettich for the amazing atmosphere in the office. Special thanks also go to Dr. Anna Polomska, Dr. Elena Moroz, Dr. Soo Hyeon Lee, Dr. Maurizio Roveri, Dr. Valentina Agostoni, Dr. Bastien Castagner, Dr. Lorine Brülisauer, Peter Tiefenböck, Michael Burger, Anna Pratsinis, Rea Signorell, Dr. Irene Pereira de Sousa, Dr. Sarah Kindgen, Aaron Schmidt, Sofia Bisso, Nadine Häni-Doëlly, Anna Kletzmeyer, Antonia Schantl and Simon Matoori for their friendship.

I am also thankful to Corinne Hofer, Murielle Delley, Alex Lauber, Elia Schneider, Samuel Hess and Basil Denzler for the cheerful atmosphere during our weekly lunches. It was nice to share thoughts and news with you.

Last but not least, I would like to sincerely thank my family and friends for their indispensable support and for their sympathy. By celebrating the good moments and cheering me up in difficult situation their presence was essential. I would like to truly thank Vittorio Sacchetti for not only reading and giving input on my manuscripts, chapters and abstracts but also for his unlimited endorsement and helping me to keep the balance between my Ph.D. thesis and life. 